\title{
Abstracts from the 52nd European Society of Human Genetics (ESHG) Conference: Oral Presentations
}

Published online: 10 October 2019

(c) European Society of Human Genetics 2019

Volume 27 I Supplement 2

The Swedish Exhibition \& Congress Center, Gothenburg, Sweden

June 15-18, 2019

Sponsorship: Publication of this supplement was sponsored by the European Society of Human Genetics. All content was reviewed and approved by the ESHG Scientific Programme Committee, which held full responsibility for the abstract selections.

Disclosure Information: In order to help readers form their own judgments of potential bias in published abstracts, authors are asked to declare any competing financial interests.

Contributions of up to EUR 10 000.- (Ten thousand Euros, or equivalent value in kind) per year per company are considered "Modest". Contributions above EUR 10 000.- per year are considered "Significant".

\section{Plenary Sessions}

PL1

Opening Plenary Lectures

\section{PL1.3}

Targeted therapy in patients with PIK3CA-related overgrowth syndrome

\section{G. Canaud}

Necker Enfants Malades hospital, Paris, France

CLOVES syndrome (congenital lipomatous overgrowth, vascular malformations, epidermal naevi, scoliosis/skeletal and spinal syndrome) is a genetic disorder that results from somatic, mosaic gain-of-function mutations of the PIK3CA gene, and belongs to the spectrum of PIK3CA-related overgrowth syndromes (PROS). This rare condition has no specific treatment and a poor survival rate. Here, we describe a postnatal mouse model of PROS/CLOVES that partially recapitulates the human disease, and demonstrate the efficacy of BYL719, an inhibitor of PIK3CA, in preventing and improving organ dysfunction. On the basis of these results, we used BYL719 to treat nineteen patients with PROS. The drug improved the disease symptoms in all patients. Previously intractable vascular tumours became smaller, congestive heart failure was improved, hemihypertrophy was reduced, and scoliosis was attenuated. The treatment was not associated with any substantial side effects. In conclusion, this study provides the first direct evidence supporting PIK3CA inhibition as a promising therapeutic strategy in patients with PROS.

G. Canaud: None.

\section{PL2}

What's New?

\section{PL2.1}

The single-cell transcriptional landscape of mammalianorganogenesis

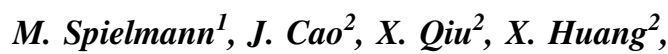
D. M. Ibrahim ${ }^{1}$, A. J. Hill', F. Zhang ${ }^{3}$, S. Mundlos ${ }^{\text {, }}$,
L. Christiansen ${ }^{3}$, F. J. Steemers ${ }^{3}$, C. Trapnell ${ }^{2}$, J. Shendure ${ }^{2}$ 
${ }^{1}$ Max Planck Institute for Molecular Genetics, Berlin, Germany, ${ }^{2}$ University of Washington, Seattle, WA, United States, ${ }^{3}$ Illumina, San Diego, CA, United States

During mammalian organogenesis, the cells of the three germ layers transform into an embryo that includes most major internal and external organs. The key regulators of developmental defects can be studied during this critical window, but conventional approaches lack the throughput and resolution to obtain a global view of the molecular states and trajectories of a rapidly diversifying and expanding number of cell types.

We have developed a three-step combinatorial barcoding method to profile single-cell transcriptomes ('sci-RNAseq3') without requiring physical isolation of each cell. We have used this new method to profile whole mouse embryos staged between 9.5 and 13.5 days of gestation with sciRNA-seq3, and created a transcriptional atlas of mouse organogenesis at single cell resolution (http://atlas.gs.wa shington.edu/mouse-rna/). We identify hundreds of expanding, contracting and transient cell types and define the corresponding sets of cell type-specific marker genes, several of which we validate by whole mount in situ hybridization. With Monocle 3, we delineate and annotate 56 single cell developmental trajectories of mouse organogenesis. We explore the dynamics of proliferation and gene expression within cell types over time, including focused analyses of the apical ectodermal ridge, limb mesenchyme and skeletal muscle. These data comprise a foundational resource for mammalian developmental biology.

This single cell atlas of the development of wild-type mice also represent a first step towards understanding pleiotropic developmental disorders at the organismal scale and provides rich opportunities for detailed investigations of subtle roles for genes and regulatory sequences involved in developmental defects.

M. Spielmann: None. J. Cao: None. X. Qiu: None. X. Huang: None. D.M. Ibrahim: None. A.J. Hill: None. F. Zhang: A. Employment (full or part-time); Significant; Illumina. S. Mundlos: None. L. Christiansen: A. Employment (full or part-time); Significant; Illumina. F.J. Steemers: A. Employment (full or part-time); Significant; Illumina. C. Trapnell: None. J. Shendure: None.

\section{PL2.2}

Chromatin 3D interactions mediate genetic effects on gene expression

O. Delaneau ${ }^{1,2}$, M. Zazhytska ${ }^{3}$, C. Borel ${ }^{2}$, G. Giannuzzi ${ }^{3}$, G. Rey ${ }^{2}$, C. Howald ${ }^{2}$, H. Ongen ${ }^{2}$, K. Popadin ${ }^{3}$, D. Marbach ${ }^{1}$, G. Ambrosini ${ }^{4}$, D. Bielser ${ }^{2}$, D. Hacker ${ }^{5}$, L. Romano-Palumbo ${ }^{2}$, P. Ribaux ${ }^{2}$, E. Falconnet ${ }^{2}$,
P. Bucher ${ }^{4}$, S. Bergmann ${ }^{1}$, S. Antonarakis ${ }^{2}$, A. Reymond ${ }^{3}$, E. Dermitzakis ${ }^{2}$

${ }^{I}$ Department of Computational Biology, University of Lausanne, Lausanne, Switzerland, ${ }^{2}$ Department of Genetic Medicine and Development, University of Geneva, Geneva, Switzerland, ${ }^{3}$ Center for Integrative Genomics, Faculty of Biology and Medicine, University of Lausanne, Lausanne, Switzerland, ${ }^{4}$ Swiss Institute for Experimental Cancer Research, Ecole Polytechnique Fédérale de Lausanne (EPFL), Lausanne, Switzerland, ${ }^{5}$ Protein Expression Core Facility, School of Life Sciences, Ecole Polytechnique Fédérale de Lausanne (EPFL), Lausanne, Switzerland

Genome-wide studies on the genetic basis of gene expression and chromatin structure have considerably advanced our understanding of the function of the human genome. However, it remains unclear how structure relates to function and here we aim at studying this by assembling a dataset combining the activity of regulatory elements (ChIP-seq for H3K4me1, H3K4me3 and H3K27ac), expression of genes (RNA-seq) and genetic variations of 317 European individuals across two cell types (Lymphoblastoid Cell Lines and Fibroblasts).

First, we show that the regulatory activity is structured in 12,583 Cis Regulatory Domains (CRDs) that are reflective of Topologically Associating Domains. In addition, we also find 25,315 significant associations between CRDs located on distinct chromosomes that form 30 Trans Regulatory Hubs (TRHs) and reflect the global chromatin organization into $\mathrm{A} / \mathrm{B}$ nuclear compartments.

Second, we show that CRDs and TRHs delimit the sets of active regulatory elements controlling the expression of most genes and are key contributors of cis and trans gene co-expression.

Finally, we show that CRDs are under strong genetic control. We discovered 58,968 chromatin peaks affected by nearby genetic variants (cQTLs). At the CRD level, this converts into genetic control of the activity of 6,157 CRDs and the structure of 110 CRDs. In practice, we estimated that $75 \%$ of these are eQTLs and showed that CRDs and TRHs capture complex regulatory networks into which the effects of eQTLs are propagated and combined to affect gene expression.

SysGenetiX grant 3826, SNSF Sinergia grant CRSI33 130326, SNSF grants 31003A_170096, 31003A_160203 and 163180 .

O. Delaneau: None. M. Zazhytska: None. C. Borel: None. G. Giannuzzi: None. G. Rey: None. C. Howald: None. H. Ongen: None. K. Popadin: None. D. Marbach: None. G. Ambrosini: None. D. Bielser: None. D. Hacker: None. L. Romano-Palumbo: None. P. Ribaux: None. E. Falconnet: None. P. Bucher: None. S. Bergmann: None. S. 
Antonarakis: None. A. Reymond: None. E. Dermitzakis: None.

\section{PL2.3}

Insights from the largest genetic study of sporadic and recurrent miscarriage

T. Laisk ${ }^{1,2}$, A. Soares ${ }^{3,4}$, T. Ferreira ${ }^{5}$, J. N. Painter ${ }^{6}$,

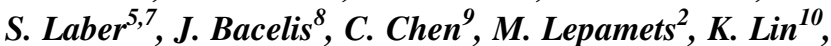
S. Liu ${ }^{11,12}$, I. Y. Millwood ${ }^{10}$, A. Ramu ${ }^{13}$, J. Southcombe ${ }^{14}$, M. S. Andersen ${ }^{15}$, L. Yang ${ }^{10}$, C. Becker ${ }^{14}$, S. D. Gordon ${ }^{6}$, J. Bybjerg-Grauholm ${ }^{16}$, Ø. Helgeland ${ }^{17}$, D. M. Hougaard ${ }^{16}$, X. Jin ${ }^{11}$, S. Johansson ${ }^{18}$, J. Juodakis ${ }^{19}$, C. Kartsonaki ${ }^{10}$, V. Kukuškina ${ }^{2}$, P. Lind ${ }^{6}$, A. Metspalu ${ }^{2}$, G. W. Montgomery ${ }^{20}$, A. P. Morris ${ }^{21}$, P. B. Mortensen ${ }^{16}$, P. R. Njølstad ${ }^{18}$, D. R. Nyholt ${ }^{22}$, M. Lippincott ${ }^{23}$, S. Seminara ${ }^{23}$, A. Salumets ${ }^{1}$, H. Snieder ${ }^{24}$, K. Zondervan ${ }^{14}$, Z. Chen ${ }^{10}$, D. F. Conrad ${ }^{13}$, B. Jacobsson ${ }^{8}$, L. Li ${ }^{25}$, N. G. Martin ${ }^{6}$, B. Neale', R. Nielsen ${ }^{26}$, R. G. Walters ${ }^{10}$, I. Granne ${ }^{14}$, S. E. Medland ${ }^{6}$, R. Mägi ${ }^{2}$, D. A. Lawlor ${ }^{4,3,27}$, C. M. Lindgren ${ }^{5,7,28}$

${ }^{1}$ Department of Obstetrics and Gynecology, University of Tartu, Tartu, Estonia, ${ }^{2}$ Estonian Genome Center, Institute of Genomics, University of Tartu, Tartu, Estonia, ${ }^{3} M R C$ Integrated Epidemiology Unit at the University of Bristol, Bristol, United Kingdom, ${ }^{4}$ Population Health Sciences, Bristol Medical School, University of Bristol, Bristol, United Kingdom, ${ }^{5}$ Big Data Institute, Li Ka Shing Center for Health for Health Information and Discovery, Oxford University, Oxford, United Kingdom, ${ }^{6}$ QIMR Berghofer Medical Research Institute, Brisbane, Australia, ${ }^{7}$ Wellcome Centre for Human Genetics, University of Oxford, Oxford, United Kingdom, ${ }^{8}$ Department of Obstetrics and Gynecology, Sahlgrenska University Hospital Östra, Gothenburg, Sweden, ${ }^{9}$ Analytic and Translational Genetics Unit, Massachusetts General Hospital, Boston, MA, United States, ${ }^{10}$ Clinical Trial Service Unit \& Epidemiological Studies Unit (CTSU), Nuffield Department of Population Health, University of Oxford, Oxford, United Kingdom, ${ }^{11} B G I-$ Shenzhen, Shenzhen, China, ${ }^{12}$ Bioinformatics Centre, Department of Biology, University of Copenhagen, Copenhagen, Denmark, ${ }^{13}$ Department of Genetics, Washington University in St. Louis, Saint Louis, MO, United States, ${ }^{14}$ Nuffield Department of Women's and Reproductive Health, University of Oxford, Oxford, United Kingdom, ${ }^{15}$ Department of Endocrinology, Odense University Hospital, Odense, Denmark, ${ }^{16}$ iPSYCH, The Lundbeck Foundation Initiative for Integrative Psychiatric Research, Copenhagen, Denmark, ${ }^{17}$ Department of Genetics and Bioinformatics, Health Data and Digitalisation, Norwegian Institute of Public Health, Oslo, Norway, ${ }^{18} \mathrm{KG}$ Jebsen Center for Diabetes Research, Department of Clinical
Science, University of Bergen, Bergen, Norway, ${ }^{19}$ Department of Obstetrics and Gynecology, Institute of Clinical Sciences, Sahlgrenska Academy, University of Gothenburg, Gothenburg, Sweden, ${ }^{20}$ University of Queensland, Brisbane, Australia, ${ }^{21}$ Department of Biostatistics, University of Liverpool, Liverpool, United Kingdom, ${ }^{22}$ School of Biomedical Sciences, Faculty of Health, Queensland University of Technology, Brisbane, Australia, ${ }^{23}$ Massachusetts General Hospital, Boston, MA, United States, ${ }^{24}$ Department of Epidemiology, University of Groningen, University Medical Center Groningen, Groningen, Netherlands, ${ }^{25}$ Department of Epidemiology \& Biostatistics, Peking University Health Science Centre, Peking University, Beijing, China, ${ }^{26}$ Department of Integrative Biology, University of California Berkeley, Berkeley, CA, United States, ${ }^{27}$ Bristol National Institute of Health Research Biomedical Research Centre, Bristol, United Kingdom, ${ }^{28}$ Program in Medical and Population Genetics, Broad Institute, Boston, MA, United States

Introduction: Miscarriage is a common complex trait that affects $10-25 \%$ of all clinically confirmed pregnancies. Miscarriage is associated with excessive bleeding, infection, depression, infertility and an increased lifetime risk of cardiovascular disease. A variety of predisposing factors have been associated with increased miscarriage risk, and it has a genetic component that thus far has remained poorly characterized. Our aim was to discover and map the maternal genetic susceptibility and underlying biology of sporadic and recurrent miscarriage.

Materials and Methods: We performed a genome wide association study including up to 69,118 sporadic miscarriage cases from seven different ancestries, 750 recurrent miscarriage cases of European ancestry, and up to 359,469 female controls. We investigated the genetic architecture of miscarriage with biobank-scale Mendelian randomization, heritability, genetic correlation and functional annotation analyses.

Results: We identified one and four loci significantly ( $p$ $<5 \times 10^{-8}$ ) associated with sporadic and recurrent miscarriage, respectively. Following functional annotation linked these associations with genes related to gonadotropin regulation, placental biology and progesterone production. We found a heritability of $29 \%$ (95\%CI 20-38\%) for miscarriage. Consistent with observational associations, we found significant genetic correlations between sporadic miscarriage and number of children $\left(\mathrm{p}=7.2 \times 10^{-9}\right)$.

Conclusion: Our results confirm miscarriage as a complex partly heritable phenotype and implicate novel biology through regulation of genes involved in gonadotropin regulation, placental biology and progesterone production.

T. Laisk: None. A. Soares: None. T. Ferreira: None. J. N. Painter: None. S. Laber: None. J. Bacelis: None. C. 
Chen: None. M. Lepamets: None. K. Lin: None. S. Liu: None. I.Y. Millwood: None. A. Ramu: None. J. Southcombe: None. M.S. Andersen: None. L. Yang: None. C. Becker: None. S.D. Gordon: None. J. Bybjerg-Grauholm: None. Ø. Helgeland: None. D.M. Hougaard: None. X. Jin: None. S. Johansson: None. J. Juodakis: None. C. Kartsonaki: None. V. Kukuškina: None. P. Lind: None. A. Metspalu: None. G.W. Montgomery: None. A.P. Morris: None. P.B. Mortensen: None. P.R. Njølstad: None. D.R. Nyholt: None. M. Lippincott: None. S. Seminara: None. A. Salumets: None. H. Snieder: None. K. Zondervan: None. Z. Chen: None. D.F. Conrad: None. B. Jacobsson: None. L. Li: None. N.G. Martin: None. B. Neale: None. R. Nielsen: None. R.G. Walters: None. I. Granne: None. S.E. Medland: None. R. Mägi: None. D.A. Lawlor: None. C.M. Lindgren: None.

\section{PL2.4}

Discovery and characterisation of 49 novel genetic disorders from analysing de novo mutations in $\mathbf{3 1 , 0 5 8}$ parent child trio exomes

\section{J. Kaplanis ${ }^{1}$, K. Samocha ${ }^{1}$, Z. Zhang ${ }^{2}$, R. Torene ${ }^{2}$, K. Arvai ${ }^{2}$, S. Lelieveld ${ }^{3}$, E. de Boer ${ }^{3}$, E. Kucuk, R. Pfundt ${ }^{3}$, L. van de Wiel ${ }^{3}$, P. Danecek ${ }^{1}$, J. McRae ${ }^{4}$, G. Gallone ${ }^{5}$, R. Eberhardt ${ }^{1}$, H. Brunner ${ }^{3}, C$. Gilissen ${ }^{3}$, K. Retterer ${ }^{2}$, M. Hurles ${ }^{1}$}

${ }^{1}$ Wellcome Sanger Institute, Cambridge, United Kingdom, ${ }^{2}$ GeneDx, Gaithersburg, MD, United States, ${ }^{3}$ Radboud Institute for Molecular Life Sciences, Nijmegen, Netherlands, ${ }^{4}$ Illumina, Inc, San Francisco, CA, United States, ${ }^{5}$ Max Planck Institute for Molecular Genetics, Berlin, Germany

Despite the rapid discovery of novel dominant developmental disorders (DD) in recent years, many more dominant DDs have yet to be discovered. Following clear consent practices, and only using aggregate, deidentified data, we pooled parent-child trio exome data from GeneDx, the Deciphering Developmental disorders study and Radboud University Medical Center to form a dataset of 31,058 DD trios. We identified 45,242 de novo mutations (DNMs), which were called with similar sensitivity and specificity across the three cohorts. We devised a novel simulationbased method to test for an enrichment of damaging DNMs in individual genes. This method scores all classes of variants on a unified severity scale based on the empiricallyestimated positive predictive value of being pathogenic, and incorporates a gene-based weighting derived from the deficit of protein truncating variants (PTVs) in the general population. Applying this method, we identified 307 significantly enriched genes exome-wide of which 49 are novel. The novel genes have a significantly larger ratio of missense to PTV DNMs compared to the known genes, suggesting they are less likely to act via haploinsufficiency. We also investigated phenotypic similarity in patients harbouring mutations in the novel genes and compared the phenotypic distribution between the known and novel genes. Overall, we explained $\sim 51 \%$ of the exome wide DNM burden within our dataset. We compared the likelihood of our observed DNMs under different underlying genetic models to estimate how many genes underpin the remaining $49 \%$ and the sample size needed to identify these.

J. Kaplanis: None. K. Samocha: None. Z. Zhang: A. Employment (full or part-time); Significant; GeneDx. R. Torene: A. Employment (full or part-time); Significant; GeneDx. K. Arvai: A. Employment (full or part-time); Significant; GeneDx. S. Lelieveld: None. E. de Boer: None. E. Kucuk: None. R. Pfundt: None. L. van de Wiel: None. P. Danecek: None. J. McRae: A. Employment (full or part-time); Significant; Illumina, Inc. G. Gallone: None. R. Eberhardt: None. H. Brunner: None. C. Gilissen: None. K. Retterer: A. Employment (full or part-time); Significant; GeneDx. M. Hurles: E. Ownership Interest (stock, stock options, patent or other intellectual property); Significant; Congenica Ltd. F. Consultant/Advisory Board; Significant; Congenica Ltd.

\section{PL2.5}

Loss of MAENLI, a newly characterized IncRNA, results in limb specific inactivation of $E N 1$ and a dorsal dimelia limb phenotype

L. Allou ${ }^{1}$, S. Balzano ${ }^{2}$, A. Magg ${ }^{1,3,4}$, M. Quinodoz ${ }^{2}$, B. Royer-Bertrand ${ }^{2}$, R. Schöpflin ${ }^{1,3,4,5}$, W. L. Chan ${ }^{3,4}$, C. E. Speck-Martins ${ }^{6}$, D. Rocha de Carvalho ${ }^{6}$, L. Farage ${ }^{7}$, C. Marques Lourenço ${ }^{8}$, S. Rajagopal ${ }^{9}$, S. Nampoothiri ${ }^{10}$, B. Campos-Xavier ${ }^{2}, C$. Chiesa ${ }^{2}$, F. Niel-Bütschi', M. Spielmann ${ }^{1}$, V. Desiniotis ${ }^{1}$, S. Unger ${ }^{2}$, C. Rivolta ${ }^{2}$, L. Wittler $^{11}$, B. Timmermann ${ }^{12}$, S. Mundlos ${ }^{1,3,4}$, A. Superti-Furga ${ }^{2}$

${ }^{1} R G$ Development \& Disease, Max Planck Institute for Molecular Genetics, Berlin, Germany, ${ }^{2}$ Medical Genetics Unit, Dept Computat Biol \& Div of Genet Medicine, Univ Hospital (CHUV) and Uni Lausanne, Lausanne, Switzerland, ${ }^{3}$ Institute for Medical and Human Genetics, Charité-Universitätsmedizin Berlin, Berlin, Germany, ${ }^{4}$ Berlin-Brandenburg Center for Regenerative Therapies, Charité-Universitätsmedizin Berlin, Berlin, Germany, ${ }^{5}$ Department of Computational Molecular Biology, Max Planck Institute for Molecular Genetics, Berlin, Germany, ${ }^{6}$ Genetic Unit, SARAH Network of Rehab Hosp, Brasilia, DF, Brazil, ${ }^{7}$ Inst Cardiol do Distrito Federal, Brasilia, DF, 
Brazil, ${ }^{8}$ Ctr Univ Estacio, Facul. Med., Ribeirao Preto, Sao Paulo, Brazil, ${ }^{9}$ Dept Med Genet, Tamil Nadu Dr. M.G.R. Med Univ, Chennai, India, ${ }^{10}$ Dept Pediat Genet, Amrita Inst, Cochin, India, ${ }^{11}$ Department of Developmental Genetics, Max Planck Institute for Molecular Genetics, Berlin, Germany, ${ }^{12}$ Max Planck Institute for Molecular Genetics, Sequencing Core Facility, Berlin, Germany

Long non-coding RNAs (IncRNAs) have been established as key regulators of gene expression, yet, we are only beginning to understand the nature and extent of the involvement of IncRNAs in human Mendelian diseases. In this study, we report three unrelated children, all from consanguineous parents, with a so far undescribed complex limb malformation consistent with a dorsal dimelia phenotype (syndactyly, mesomelic shortening, triangular tibia, absent patella, vertically positioned feet and nails on the palmar side of digits). Array-CGH identified overlapping homozygous deletions located $200 \mathrm{~kb}$ upstream of Engrailed-1 (EN1), a homeobox containing gene involved in brain and limb development. CRISPR/Cas 9 induced deletions of the homologous region in mice resulted in a double dorsal limb phenotype recapitulating the human malformation due to a complete loss of Enl expression specifically in the limb. Using genome-wide epigenetic studies in the developing mouse and human limbs, we characterized a new lncRNA (Maenli - Master regulator of engrailed in the limb) in the deleted region consisting of an approximately $1 \overline{\mathrm{kb}} 4$ exon transcript. Detailed characterization in vivo including promoter deletion, promoter inversion, and insertion of a stop cassette showed that Maenli specifically regulates Enl expression in the limb but not at other sites such as the brain. We also show that the inactivation of Maenli is the cause of the double dorsal limb phenotype and as such the first description of an lncRNA in a human developmental disorder. Our study highlights the involvement of lncRNAs as a new disease mechanism in congenital malformations.

L. Allou: None. S. Balzano: None. A. Magg: None. M. Quinodoz: None. B. Royer-Bertrand: None. R. Schöpflin: None. W.L. Chan: None. C.E. Speck-Martins: None. D. Rocha de Carvalho: None. L. Farage: None. C. Marques Lourenço: None. S. Rajagopal: None. S. Nampoothiri: None. B. Campos-Xavier: None. C. Chiesa: None. F. Niel-Bütschi: None. M. Spielmann: None. V. Desiniotis: None. S. Unger: None. C. Rivolta: None. L. Wittler: None. B. Timmermann: None. S. Mundlos: None. A. Superti-Furga: None.

\section{PL2.6}

Whole-genome sequencing of rare disease patients in a national healthcare system
L. Raymond, on behalf of The NIHR BioResource and the 100,000Genomes Project - Rare Diseases Pilot

\section{Cambridge Institute for Medical Research, Cambridge, United Kingdom}

To study genetic variants underlying unresolved Mendelian disorders and to improve interpretation of pathogenic variants, we sequenced the genomes of 13,000 individuals with rare diseases and relatives to an average 30x coverage. Participants were recruited in the UK using approved eligibility criteria for 15 different rare disease domains. We identify over 172 million unique short variants $(91.5 \%$ SNVs, 3\% insertions, $6 \%$ deletions) in the 10,259 genetically independent samples. Pertinent findings in over 2,000 diagnostic-grade genes with 1,106 unique causal variants (733 SNVs, 263 indels, 104 large deletions, 6 other structural variants) affecting 304 genes were reported. Diagnostic yields varied across disease domains from 1.6-54\%. Using a Bayesian genetic association method, BeviMed, we identified evidence (posterior probability $>0.75$ ) for 99 genetic associations between rare variants and groupings of patients with similar phenotypes. Of these 61 had firmly established evidence for causality. We identified novel variants in regulatory elements of ARPC1B, GATA1, $L R B A$ and $M P L$. We used Telomerecat software to estimate telomere lengths (TL). We identified rare variants in TERT and $D K C 1$ in patients with extremely short TLs. We demonstrate how rare variants in previously reported GWAS loci facilitate identification of novel monogenic disease genes. We demonstrate the feasibility of using WGS across a national healthcare system to deliver a diagnosis for patients, to identify novel associations and complex genetic architecture in rare diseases.

L. Raymond: None.

\section{PL3}

Mendel Award Lecture

\section{PL3.1A \\ 25 Year Genomic Odyssey}

C. Venter

\section{J. Craig Venter Institute, San Diego, CA, United States}

In 1995 J. Craig Venter, Ph.D. and team at the Venter Institute sequenced the first genome in history followed five years later when Celera Genomics published the first draft human genome which was a composite of five individual's genomes. In 2007 he and his team at the J. Craig Venter Institute published the first complete diploid human 
genome, which was his genome. While these genomes were an important starting point for research, they were not enough to change health care. Hundreds of thousands of people need to have their own genome sequenced and done so at a complete and accurate level to have sufficient data to interpret the genome. Cost was prohibitive and the technology was not yet robust enough in 2007 to make this a reality. Three years ago, this changed with the advent of new sequencing technology and improved bioinformatics and machine learning tools. Dr. Venter and his team continue their work today which was begun decades ago in comparing genotype to phenotype to better understand human biology, health and disease. He will discuss ongoing research which the primary objective is to realize the full impact of genomics prediction, to have a thorough understanding of the DNA software and how it codes for the wide range of human phenotypes and abilities. He and his team have a 25-year track record of continually increasing the information content that can be derived from the human genome. More recently, we have merged this expertise with an extensive phenotyping platform and machine learning techniques to demonstrate its predictive power to improve health outcomes. Dr. Venter will also discuss his pioneering work in synthetic biology. In 2010, he and his team at the Venter Institute became the first to successfully construct 'synthetic life'-putting humankind at the threshold of the most important and exciting phase of biological research, one that is enabling us to actually write the genetic code for designing new species that can be used in many ways for the betterment of mankind. The science of synthetic genomics is and will continue to have a profound impact on human existence, including new chemical and energy generation, human health and medical advances, clean water and food production, positive environmental impact, and possibly even our evolution.

C. Venter: None.

\section{PL4}

\section{ESHG Award Lecture}

\section{PL4.1}

We and our second genome: two key players in common complex diseases

\section{Wijmenga}

Lodewijk Sandkuijl Professor of Human Genetics, UMCG Groningen, Groningen, Netherlands

The manifestation of common complex diseases is the outcome of interaction of many different genetic factors in conjunction with non-genetic environmental factors. In recent years, the knowledge on our genome has been greatly expanded, as thousands of genetic variants have been associated to hundreds of complex traits and diseases. In addition, we have seen increasing awareness of the important role of another set of genomes in our body, also known as human microbiome or our second genome. Our gut microbiome encodes some 150 times more genes that the host genome. While the human genome is set at birth, the gut microbiome can undergo dynamic changes over the course of an individual's life. By making use of the population-based LifeLines-DEEP cohort, we have shown that the inter-individual variation of the gut microbiome captures quite some of the variation contributed by genetic and non-genetic environmental factors, thereby contributing to the susceptibility to complex diseases. Since the gut microbiome can be modified, this offers opportunities for future therapeutic interventions. However we first need to understand if changes in the microbiome cause disease, or whether the microbial changes are the consequence of disease. To move towards causality we applied, as a proofof-concept, Mendelian Randomization and were able to show that microbial changes are causal for type 2 diabetes and metabolic traits. Furthermore, we are developing a guton-a-chip, a sophisticated microfluidic device to study the interplay between the human host, the gut epithelial barrier, the immune system and the gut microbiome.

C. Wijmenga: None.

\section{Concurrent Symposia}

\section{S01}

Updates in psychiatric genetics

S01.2

Genome wide patterns of structural mutation and selection guide the identification of cis-regulatory structural variants in autism

\author{
J. Sebat ${ }^{1}$, P. Tandon ${ }^{2}$ \\ ${ }^{1}$ La Jolla, CA, United States, ${ }^{2}$ UCSD, La Jolla, CA, United \\ States.
}

Genome rearrangement is a driving force in human evolution and a contributor to complex traits and diseases. Structural Variants (SVs), including deletions and duplications of chromosomal regions, exhibit significant clustering in the genome, reflecting regional differences in mutation rate and the action of natural selection. Our work develops a multi-scale regression model to predict $\mathrm{SV}$ mutation rates based on DNA sequence motif features. By applying our random mutation model to population data, we identify 
categories of functional elements that are under significant SV constraint, including enhancers and promoters and UTRs. We then apply SV constraint to estimate the deleteriousness of SVs in genome sequencing studies, and we demonstrate that a proportion of the inherited risk for autism is attributable cis regulatory structural variants (CRE-SVs).

P. Tandon: None.

S01.3

Updates in clinical applications of psychiatric genetics

J. Austin ${ }^{1,2}$

${ }^{1}$ University of British Columbia, Vancouver, BC, Canada, ${ }^{2} B C$ Mental Health and Addictions Research Institute, Vancouver, BC, Canada

Background: We now have a substantial and growing list of genetic variations - including both single nucleotide polymorphisms and copy number variations that we can confidently identify as contributing to the etiology of conditions like schizophrenia, bipolar disorder and depression. This progress is accompanied by a need for urgent attention to the question of how to apply this knowledge clinically in such a way as to promote the best possible outcomes for those who live with psychiatric disorders and their families.

Methods: In this presentation, the outcomes of applying our developing knowledge of the etiology of psychiatric disorders in the clinical setting will be reviewed. For example, we will explore the psychological importance for people with psychiatric illness and their families of understanding cause of illness, and in particular, the psychological and behavioural ramifications of understanding that there is a genetic contribution to these conditions. The impact of counseling environment, timing of family history documentation, and risk communication on patient outcomes of psychiatric genetic counseling will be discussed.

Results: Through presentation of both quantitative and qualitative, narrative-based data, some of the key ramifications of applying psychiatric genetics data in clinical practice will be highlighted.

Conclusion: These data suggest factors that may be important to consider when implementing genetic data in clinical practice in order to promote the best possible outcomes for patients with psychiatric disorders and their families.

J. Austin: None.

S02

Finding the strengths that make cancer cells weak
S02.3

\section{Immunotherapy in cancer}

\section{K. Serre ${ }^{1}$, S. Mensurado ${ }^{1}$, A. Simões ${ }^{1,2}$, D. Correia ${ }^{1,2}$, B. Silva-Santos ${ }^{1,2}$}

${ }^{1}$ Instituto de Medicina Molecular| JLA, Lisbon, Portugal, ${ }^{2}$ Lymphact - Lymphocyte Technologies S.A., Lisbon, Portugal

Immunotherapy is a game changer in the treatment against cancers. In particular, the immune checkpoint blockers that unleash host anti-tumour lymphocytes, or the adoptive transfer of anti-tumour $\mathrm{T}$ cells have provided remarkable results. Regrettably, although these treatments yield extraordinary hope, only a small fraction of patients fully respond. Resistance to treatment can be intrinsic to tumour cells, but it is also conferred by the tumour microenvironment. We will discuss the principles of immunotherapy exemplified with current options and results in various types of cancers and envisage the future avenues. Then, I will present two ongoing research lines in our laboratory.

First, I will present a cellular immunotherapy product composed of gamma-delta $\mathrm{T}$ cells that selectively target malignant (but not normal) leukocytes. This subset expresses the Vdelta1 TCR and a broad repertoire of natural cytotoxicity receptors (NCRs), such as NKp30 and NKp44, which recognize transformation-induced ligands like B7H6. We will discuss the research behind these lymphocytes, termed Delta One T (DOT-) cells, that leads to their proposed testing in leukaemia clinical trials by the end of the year.

Second, I will provide evidence that we can induce tumour regression in mouse model of breast cancer, in a manner that depends strictly on macrophages. Macrophages, which can represent over 50\% of the tumour immune infiltrate, are usually associated with poor prognosis. Although these myeloid cells can also act as powerful anti-tumour effectors, the effective induction of anti-tumour functions in macrophages remains a major scientific and clinical challenge. Thus, our data will shed new light on the remarkable potential that shaping macrophages may offer to design novel avenues for immunotherapy.

K. Serre: None. S. Mensurado: None. A. Simões: None. D. Correia: None. B. Silva-Santos: None.

S03

RNA mis-splicing dynamics, diagnosis and treatment

S03.1

Minor spliceosome and disease 


\section{J. Frilander}

\section{University of Helsinki, Helsinki, Finland}

The minor spliceosome, which is also known as the U12dependent spliceosome, excises rare U12-type introns during the nuclear mRNA processing step. U12-type introns are characterized by highly conserved $5^{\prime}$ splice site and branch point sequences. Human genome contains approximately 700 genes carrying U12-type introns, typically one intron in each gene (1). Unlike the normal U2-type introns, the U12-type introns are not typically alternatively spliced to produce multiple protein-coding mRNA isoforms. Instead, they show elevated level of intron retention in RNAseq data that has been interpreted as less efficient splicing of this type of introns, and a possibility for ratelimiting regulation of the genes containing U12-type introns $(2,3)$.

Several human diseases have been associated with the minor spliceosome. While only two diseases have been reported to result from mutations in the U12-type intron splice sites, six diseases feature mutations in the specific components of the minor spliceosome (4). Of these, congenital mutations in the U4atac snRNA lead to Microcephalic osteodysplastic primordial dwarfism/Taybi-Linder Syndrome (MOPD1/TALS), Roifman syndrome (RFMN), and Lowry-Wood Syndrome (LWS). In U12 snRNP, mutations in U12 snRNA lead to Early-Onset Cerebellar Ataxia (EOCA) and mutations in RNPC3 to Isolated growth hormone deficiency type I (IGHD). Additionally, somatic mutations in ZRSR2 have been associated with Myelodysplastic syndrome (MDS). Additionally, several neuronal diseases such as SMA and certain forms of ALS show defects in the splicing of U12-type introns (5).

I will give a brief overview of the mechanism and significance of the system with an emphasis to diseases linked to this mRNA processing machinery. I will also discuss the consequences of different mutations in generating diverse defects at the level of individual transcripts, and their association with different disease phenotypes.

\section{References:}

1. Turunen et al. (2013). Wiley Interdiscip Rev RNA 4, $61-76$.

2. Niemelä \& Frilander (2014). RNA Biology 11, $1325-1329$.

3. Oghabian et al. (2018). BMC Bioinformatics 19, 130.

4. Verma et al. (2018). Sem Cell Dev biol 79, 103-112.

5. Jutzi et al. (2018). Cell Stress 2, 40-54.

M.J. Frilander: None.

\section{S03.2}

Dynamic mutations and RNA mis-splicing in disease
M. Swanson, C. A. Nutter, J. L. Bubenik, R. Oliveira, L. J. Sznajder, B. M. Kidd

University of Florida, Gainesville, FL, United States

Repetitive elements, including transposable elements and tandem repeats, are prominent structural features of the human genome. Among the latter repetitive class, microsatellites or short tandem repeats (STRs, $\leq 10 \mathrm{bp}$ ) are particularly dynamic sequences and are prone to expansion mutations that cause $>30$ neurological and neuromuscular diseases. While STR expansions may occur in both the coding and noncoding regions of genes, pathomechanistic studies of noncoding expansions led to the discovery of STR-induced RNA-mediated and protein-mediated toxicity mechansims, due to either RNA-binding protein (RBP) sequestration or repeat-associated non-AUG (RAN) translation (RAN), respectively. To gain further insights into disease pathology and also provide mammalian platforms for drug testing, transgenic mouse models for these diseases have often employed heterologous promoters and gene contexts but this strategy fails to reproduce the tissue specificity, developmental timing and spatial expression of the affected endogenous gene. Here, we report the development of a new generation of mouse knockin models using rolling circle amplification (RCA) in vitro to generate large stable repeat expansions combined with CRISPR/ Cas9 genome editing for the neuromuscular disease myotonic dystrophy type 1 , which is caused by a CTG expansion $\left(\mathrm{CTG}^{\mathrm{exp}}\right)$ in the $3^{\prime} \mathrm{UTR}$ of the DMPK gene. Dmpk CTG $^{\text {exp }}$ HDR templates resulted in knockin mice carrying a range of expansion mutations due to repeat contractions in vivo and DM1-relevant disease manifestations, including nuclear RNA foci and developmental dysregulation of RNA alternative splicing, were detectable in both tissues and primary cells. Our results demonstrate that microsatellite expansions can be effectively introduced into affected gene loci resulting in knockin mice that serve as multisystemic experimental and therapeutic platforms for STR expansion diseases.

M. Swanson: F. Consultant/Advisory Board; Modest; Locana Bio. C.A. Nutter: None. J.L. Bubenik: None. R. Oliveira: None. L.J. Sznajder: None. B.M. Kidd: None.

S03.3

Restoring splicing defects by antisense oligonucleotide therapy

R. W. Collin

Radboud university medical center, Nijmegen, Netherlands 
Antisense oligonucleotides (AONs) are small DNA/RNA molecules able to modulate pre-mRNA splicing, including the correction of aberrant splicing processes due to genetic mutations. AONs are employed for a variety of inherited diseases, including Duchenne muscular dystrophy, spinal muscular atrophy and various metabolic disorders. In this presentation, I will mainly focus on the use of AONs for inherited retinal diseases (IRD), a group of disorders that is highly heterogeneous, both at the genetic and clinical level. Our proof-of-concept work on the correction of a recurrent splicing defect underlying CEP290-associated Leber congenital amaurosis (LCA), one of the most severe subtypes of IRD, has led to the initiation of a clinical trial, with recently announced promising results, demonstrating safety and in some subjects efficacy upon intra-ocular AON delivery. We expanded the use of AONs for the treatment of other subtypes of IRD, including Stargardt disease (STGD1) which is caused by $A B C A 4$ mutations. Employing midigene splice assays, as well as using patient-derived fibroblast cells, we have identified the splice defects that are associated with a large group of recently identified deepintronic $A B C A 4$ mutations. In some cases, induced pluripotent stem cell (iPSC) technology - differentiation of iPSC cells towards a retinal fate - was needed to demonstrate retina-specific splicing defects. In all cases, AONs were designed that were able to rescue the aberrant splicing events. Another, recurrent, ABCA4 variant (c.768G $>$ T) leads to an exon extension of 35 nucleotides (nt), thereby disrupting the reading-frame. Intriguingly, also the 35-nt extension caused by the common c.768G $>\mathrm{T}$ variant could be rescued by blocking the alternative splice donor site in intron 6 , forcing the spliceosome to use the original splice donor site of exon 6. Taken together, AONs appear to be an effective and versatile tool to correct different types of splice defects associated with STGD1. Given the promising data obtained so far in a clinical trial using AONs for the treatment of CEP290-associated LCA, AONs may serve as a more broadly applicable therapeutic strategy for various subtypes of IRD.

R.W. Collin: E. Ownership Interest (stock, stock options, patent or other intellectual property); Modest; ProQR Therapeutics.

\section{S04}

\section{An update on kidney research}

\section{S04.1}

Polycystic kidney disease and ciliopathies

\section{Bergmann}

Department of Medicine, University Hospital Freiburg, Freiburg, Germany

Cystic kidneys are common causes of end-stage renal disease, both in children and in adults. Autosomal dominant polycystic kidney disease (ADPKD) and autosomal recessive polycystic kidney disease (ARPKD) are cilia-related disorders and the two main forms of monogenic cystic kidney diseases. ADPKD is a common disease that mostly presents in adults, whereas ARPKD is a rarer and often more severe form of polycystic kidney disease (PKD) that prenatally may result in Potter's oligohydramnios sequence. Early and severe forms of PKD are quite common. About the same number of children do carry mutations in the major genes for ARPKD and ADPKD. The latter often arise de novo or may affect both disease alleles in a recessive mode. In addition, mutations in several other cystogenes can phenocopy early and severe PKD. A comprehensive knowledge of disease-causing genes is essential for the correct diagnosis, recurrence risk and parental counselling. Cell biological and clinical research approaches have expanded our knowledge of its pathogenesis and revealed some mechanistic overlap between the different forms of PKD. A reduced 'dosage' of PKD proteins is thought to disturb cell homeostasis and converging signalling pathways, such as $\mathrm{Ca} 2+$, cAMP, mTOR, WNT, VEGF and Hippo signalling, and could explain the more severe clinical course in some patients with PKD. Genetic diagnosis might benefit families and improve the clinical management of patients, which might be enhanced even further with emerging therapeutic options. In this talk, I will provide an overview of the current knowledge of PKD and its treatment.

C. Bergmann: A. Employment (full or part-time); Significant; Bioscientia/Sonic Healthcare. E. Ownership Interest (stock, stock options, patent or other intellectual property); Significant; Sonic Healthcare. F. Consultant/ Advisory Board; Modest; Alexion, Otsuka, Merck, PTC, Bristol-Myers Squibb.

\section{S04.2}

New insights in the genetics of hereditary nephrotic syndromes

\section{Antignac}

Imagine Institute, Paris, France

Nephrotic syndrome (NS) is a clinical entity characterized by massive proteinuria, hypoalbuminemia and hyperlipidemia. Inherited forms of steroid-resistant nephrotic syndrome (SRNS) (either isolated or associated with extra-renal disorders), which frequently lead to end-stage renal failure 
in children or young adults, appeared to be genetically heterogeneous and pinpointed the crucial role of the podocyte, a highly specialized glomerular epithelial cell, in the regulation and function of the glomerular filtration barrier. Indeed, most of the 50 genes identified to date in SRNS encode proteins that are expressed in the podocyte and that are structural podocyte proteins or are involved in key biological pathways such as regulation of actin or microtubule dynamics and organization, vesicular trafficking and cellular recycling processes... More recently, important progresses have been made in deciphering the genetic bases of Galloway-Mowat syndrome (GAMOS) associating SRNS with neurological defects and microcephaly. This syndrome raises the fascinating question of the molecular pathways that could be common to brain and kidney, in line with the growing evidence that podocytes and neurons share a large set of molecular processes essential to development of the glomerulus and the nervous system. Disease-causing mutations have been identified in 8 genes to date: WDR73 encoding a WD40 repeat-containing protein required for regulation of cell survival and microtubule cytoskeleton dynamics, NUP133 and NUP107 encoding nucleoporins, and very interestingly 5 genes encoding proteins involved in tRNA posttranscriptional modifications (4 proteins of the KEOPS complex and WDR4, responsible for N-6threonylcarbamoylation $\left(\mathrm{t}^{6} \mathrm{~A}\right)$ at position 37 in $\mathrm{ANN}$ decoding tRNAs and 7-methylguanosine modification respectively. These data highlight the vulnerability of podocytes, as neurons, to cellular defects due to RNA metabolism alterations.

\section{Antignac: None.}

\section{S04.3}

\section{CRISPR Gene Editing in Human Organoids for Inherited Renal Diseases}

\section{B. Freedman}

\section{University of Washington, Seattle, WA, United States}

Kidneys cannot naturally regenerate lost tissue, and few preventive medications exist, limiting treatment options to temporary salves of dialysis or transplant with substantial side effects. We have developed a simple method to differentiate human pluripotent stem cells into intricately patterned, multi-segment organoids that resemble kidney tissues. These organoids form via a developmental pathway that induces the nephron progenitor cell, which gives rise to podocytes, proximal tubules, and distal tubules along a proximal-to-distal axis.

While beautiful, how to translate organoids into innovative therapies for organs as complex as human kidneys remains a critical question. To address this challenge, we have applied CRISPR gene editing to reveal disease mechanisms in organoids and test therapeutic interventions. Mutations associated with polycystic kidney disease or cilia cause organoid tubules to swell thousands of times in size, producing large, fluid-filled cysts of centimeter diameters. In contrast, mutations associated with podocytes, the filtering cells of the kidney, do not affect tubules but cause junctional deformities that explain urinary defects in vivo. Harnessing the power of automation, scRNA-seq analysis of organoids reveals sixteen different cell types, and identifies a novel three-gene signature of glomerular disease that appears in human patients.

To improve organoid function and seek therapies, thousands of organoids can be manufactured simultaneously in high throughput screening formats, and analyzed for multidimensional phenotypes of differentiation, toxicity, and disease. Screening reveals treatments that dramatically increase the vascular endothelium, and a surprising role for non-muscle myosin in cystogenesis, which can be targeted pharmacologically targeted to modulate cystogenesis. Organoids with live fluorescence reporters and in microfluidic kidney-on-a-chip formats provide next-generation platforms for phenotypic screening and illumination of intracellular mechanisms at the tissue scale.

Collectively, our findings delineate key strategies and focus areas for advancement of kidney therapeutics using human organoids as surrogates for drug discovery, gene therapy, and regeneration.

\section{B. Freedman: None.}

\section{S05}

Genome editing

S05.1

CRISPR single-cell sequencing: Toward functional biology in high throughput

\section{Bock ${ }^{1,2,3,4}$}

${ }^{1}$ CeMM Research Center for Molecular Medicine of the Austrian Academy of Sciences, Vienna, Austria, ${ }^{2}$ Department of Laboratory Medicine, Medical University of Vienna, Vienna, Austria, Vienna, Austria, ${ }^{3}$ Max Planck Institute for Informatics, Saarbrücken, Germany, ${ }^{4}$ Ludwig Boltzmann Institute for Rare and Undiagnosed Diseases, Vienna, Austria

International consortia have mapped the human transcriptome and epigenome in hundreds of cells types (ENCODE, IHEC, BLUEPRINT, etc.) and thousands of individuals (GTEx, GoDMC etc.). These maps are being refined by 
ongoing single-cell sequencing projects (HCA, HuBMAP, etc.), which will eventually give rise to a comprehensive catalog of all cells in the human body. However, we are lagging behind with our ability to assign biological functions to the observed gene regulatory patterns.

CRISPR-based genetic screens have the potential to accelerate functional and gene-regulatory studies, but current methods have inherent limitations. Widely used pooled screens are restricted to simple readouts including cell proliferation and sortable marker proteins. Arrayed screens allow for comprehensive molecular readouts such as transcriptome profiling, but operate at much lower throughput.

To overcome these limitations, we combined pooled CRISPR screening with single-cell RNA sequencing into a broadly applicable workflow, directly linking guide RNA expression to transcriptome responses in thousands of individual cells (Datlinger et al. 2017 Nature Methods). Our CROP-seq method enables pooled CRISPR screens with single-cell transcriptome resolution in a way that scales well in the number of cells (up to millions using combinatorial indexing) and target genes (up to genome-wide).

We and many other labs have found CROP-seq broadly useful for studying biological mechanisms that are difficult to reduce to a simple readout needed for classical pooled screens. Applied to heterogeneous cell populations and ex vivo tissue (e.g., in combination with Cas9-expressing mice), CROP-seq can identify cell-type-specific regulation in entire organs and uncover cellular differentiation hierarchies.

Given the increasing throughput of single-cell transcriptomics and the advent of single-cell multi-omics technology (reviewed in: Bock et al. 2016 Trends in Biotechnology), CROP-seq enables deep profiling of large CRISPR libraries and constitutes a powerful method for functional biology at scale.

Funding: C.B. is supported by an ERC Starting Grant ( $n$ - 679146) of the European Union and by a New Frontiers Group award of the Austrian Academy of Sciences.

C. Bock: E. Ownership Interest (stock, stock options, patent or other intellectual property); Significant; Aelian Biotechnology. F. Consultant/Advisory Board; Significant; Aelian Biotechnology.

\section{S05.3}

Advances in therapeutic CRISPR/Cas9 genome editing

\section{G. Schwank}

\section{IMHS, Zurich, Switzerland}

The CRISPR-Cas genome-editing tool holds great promise for treatment of genetic disorders. Clinical translation of these targeted nucleases, however, is hampered by the low frequency of precise repair of Cas9-induced DNA doublestranded breaks via homologous recombination from template DNA. This problem is in particular eminent in non-dividing tissues such as the adult liver. In my presentation I will provide insights into our efforts of developing in vivo gene editing approaches for monogenetic liver diseases. I will present a study where we circumvented the problem of low rates of homologous recombination in hepatocytes by applying CRISPRassociated base editors. Base editors enable direct conversion of C.G to T.A base pairs and vice versa by base deamination and independent of DNA break formation and homologous recombination from template DNA. When targeting a mouse model for the metabolic liver disease Phenylketonuria, systemic delivery of base editors via AAV resulted in gene correction rates that fully restored physiological blood phenylalanine levels. We observed correction rates above $60 \%$, and restoration of the phenylalanine hydroxylase enzyme activity. Our findings suggest the feasibility of using CRISPR-associated base editors to repair genetic diseases in vivo in adult patients.

G. Schwank: None.

\section{S06}

Thank you for the Variant (a personal utility tale)

\section{S06.2}

Genetic profiling in primary care: triggers and impact on risk-reducing behaviour

\section{N. Qureshi}

NIHR School of Primary Care Research, University of Nottingham, Nottingham, United Kingdom

Currently access to genomic profiling in Britain is most commonly through direct-to-consumer testing, such as available at high street retailers. Our studies indicate that family history is an important trigger for many people to engage with testing but discordance between an individual's genomic profile results and their family history can cause confusion and adversely impact upon their risk-reducing lifestyle behaviours, such as weight loss and smoking cessation. Often those individuals taking up direct-toconsumer testing have already optimised lifestyle riskreducing health behaviours. Of concern is that positive genomic results may precipitate a fatalistic attitude and negative health beliefs about improving health. Similarly, negative results may trigger apathy towards risk-reducing behaviours, even though these individuals may still be at high risk of disease as a result of avoidable risk factors. However, non-modifiable risk factors, such as genomic 
profiling and family history, can influence (modifiable) lifestyle risk reducing behaviours. One possible driver for lifestyle behaviour change is the anxiety associated with positive genomic test results. This has been demonstrated in studies incorporating genetic risk assessment in primary care. Primary healthcare professionals will interact with patients who have engaged in direct-to-consumer genomic testing, and therefore have an opportunity to identify why the patient has taken up the test and the impact it has had on modifiable risk-reducing behaviour in those individuals.

\section{N. Qureshi: None.}

S06.3

What will this genetic result mean for my baby?

\section{Henneman}

\section{Amsterdam UMC, VUMC, Amsterdam, Netherlands}

Technological advancement, including next generation sequencing, increases the possibilities for populationbased preconception, prenatal and newborn screening. A larger number and broader range of conditions are added to tests such as preconception carrier screening panels and non-invasive prenatal testing (NIPT), which may benefit couples' reproductive autonomy. In newborn bloodspot screening (NBS), expansion is facilitated by increased clinical understanding of disorders and the availability of new treatments, which can improve early diagnosis to prevent irreversible health damage in children.

While the expansion of screening tests could be seen as beneficial, it also raises new ethical, psychological and societal issues. Many of the screening developments are technology-driven, and it is important to attune to parents' demand. With the increasing number and type of conditions for which testing can be offered, the number of false positive and additional (unclear) findings increases, thereby complicating information provision and counseling for informed decision making. Abnormal (positive) genetic screening test results understandably have great impact on parents, but especially uncertain findings can result in high parental anxiety.

In an expansion of the scope of screening the benefits should outweigh the harms (proportionality). The widening scope of these technologies should therefore be considered acceptable and meaningful, including the perspectives of its users. In my presentation I will address the challenges with expanded screening in the context of preconception, prenatal and neonatal screening, and particularly focus on the parents' perspective.

L. Henneman: None.
S07

Polygenic risk scores coming of age

\section{S07.2}

Polygenic risks and their impact on behavior

\section{S. Ripatti ${ }^{1,2,3}$}

${ }^{1}$ Institute for Molecular Medicine Finland (FIMM), Helsinki, Finland, ${ }^{2}$ Department of Public Health, University of Helsinki, Helsinki, Finland, ${ }^{3}$ Broad Institute of MIT and Harvard, Cambridge, MA, United States.

While polygenic risk scores (PRS) have shown promise in risk stratification in common diseases, evidence about their value in predicting future cases alongside clinical risk factors is scarce. In this talk, I will discuss strategies for combining established clinical risk factors and genetic risk evaluation, and show how PRSs for common diseases, and behavioral and biomarker traits behave in Finnish biobank data, including the FinnGen study with 150,000 individuals and up to 46 of registry-based follow-up. In addition, the talk highlights why PRSs could provide a useful tool for stratified preventive actions such as cancer screening.

There is also limited experience on returning the polygenic risk information in a routine healthcare setting; in the second part of the talk I will discuss how returning genetic and clinical risk motivates treatment and behavioral changes. In the GeneRISK Study, we randomly sampled 7,350 middle-aged individuals, evaluated their traditional coronary heart disease (CHD) risk factors and integrated them with a PRS for CHD. We then returned 10-year risk estimates back to the study participants through an interactive web-based tool, KardioKompassi. Returning this personalized risk information resulted in favourable changes in behavior, attitudes, and risk factor levels. The talk will also display early results from pilot studies of providing genetic risk information in clinical settings in Finland and in Estonia.

\section{S. Ripatti: None.}

\section{S07.3}

\section{Polygenic risk scores in prostate cancer}

\section{R. Eeles}

The Institute Of Cancer Research \& Royal Marsden NHS Trust, London, United Kingdom

There are over 1 million cases of prostate cancer $(\mathrm{PrCa})$ worldwide per year and it is becoming the commonest solid cancer in men in the Western world. There is evidence for genetic predisposition to $\mathrm{PrCa}$ from case-control 
epidemiological studies, and twin studies. The higher risks found with monozygotic versus dizygotic twins support the hypothesis that familial aggregation results from shared genetic rather than just environmental factors. Men of African ancestry have nearly twice the incidence rates of Caucasians.

Genetic predisposition to common cancers is mainly due to a mixture of contribution from both common variants with slightly increased risks (generally a relative risk of 2fold or less) and rarer, higher risk variants which confer higher risks (usually 3-8 fold). In PrCa predisposition, we now have molecular evidence that both models exist; furthermore, it is the common cancer with the highest proportion of a common genetic variation component.

Most of the rarer variants predisposing to $\mathrm{PrCa}$ are in the DNA repair pathway. Consistent evidence is now emerging that BRCA mutation carriers who develop PrCa also develop worse disease with a higher proportion of nodal and metastatic spread, and have a poorer survival. More recently, evidence has also emerged that other genes in this pathway also predispose to $\mathrm{PrCa}$ and that the resultant $\mathrm{PrCa}$ disease is more aggressive.

However, the largest component of genetic predisposition to $\mathrm{PrCa}$ is due to commoner genetic variants (occurring in $>5 \%$ of individuals). Each confers a small relative risk, but because they are numerous and their risks are multiplicative, they can combine to contribute quite substantial relative risks. In a study of 140000 individuals using a chip, the OncoArray, with over 500000 SNPs, in Caucasians, we discovered 65 new SNPs and when combined with previous findings, a total of 170 SNPs explain $38.5 \%$ of the familial relative risk of PrCa. In risk stratification, men in the top $1 \%$ of the genetic risk score group have a relative risk of 5.7 fold for developing PrCa compared with the median risk group. These results currently apply to Caucasian populations and a multiethnic analysis is on-going.

In the talk, I will discuss how common SNP profiling is being studied to stratify populations for targeted PrCa screening programmes and how such SNP profiles can modify the relative PrCa risks from the rarer variants.

R. Eeles: None.

\section{S08}

\section{Beware of the transposons}

\section{S08.1}

YY1: an enduring repressor of $\mathrm{L} 1$ retrotransposition during human neurodevelopment

\section{G. J. Faulkner}

University of Queensland, Brisbane, Australia
Nearly $20 \%$ of the human genome is composed of LINE-1 (L1) retrotransposons. However, in each of us, fewer than $10 \mathrm{~L} 1$ copies have significant potential to mobilise in germinal and somatic cells. Germline L1 mutagenesis is a known source of disease, whereas the consequences of somatic L1 retrotransposition in tumours and the brain are mostly unclear. In this talk, I will present results showing YY1 (Yin Yang 1) mediates L1 repression via DNA methylation. Mobile L1s lacking a YY1 binding site, a motif conserved for $>70 \mathrm{Myr}$ of human evolution, are severely hypomethylated during neurodevelopment, and can "jump" to cause somatic L1 mosaicism in the brain. YY1 is required for full-length L1 transcription, meaning L1 cannot escape its repressor. These experiments elucidate a major pathway controlling L1 mutagenesis in human cells, and explain how L1 can evade silencing to retrotranspose in the brain.

\section{G.J. Faulkner: None.}

\section{S08.2}

\section{Alu elements and cellular RNA metabolism}

\section{E. Maquat}

\section{University of Rochester, Rochester, NY, United States}

Primate-specific Alu SINEs as well as rodent-specific B and ID (B/ID) SINEs can promote mRNA export from the nucleus to the cytoplasm, translation in the cytoplasm, and/ or Staufen-mediated mRNA decay (SMD) when present in mRNA 3'UTRs. The transposable nature of SINEs, their presence in long noncoding RNAs, their interactions with Staufen (STAU), and their rapid divergence in different evolutionary lineages suggest they could have generated substantial modification of post-transcriptional gene-control networks during mammalian evolution. Some of the variation in SMD regulation produced by SINE insertion might have been similarly adaptive in separate mammalian lineages, leading to parallel evolution of the STAU network by independent exaptation of SINEs. To explore this, we searched for orthologous gene pairs, each carrying a species-specific $3^{\prime}$ UTR SINE and each regulated by SMD, by measuring changes in mRNA abundance after individual depletion of two SMD factors, STAU1 and UPF1, in both human and mouse myoblasts. We identified and confirmed orthologous gene pairs with $3^{\prime}$ UTR SINEs that have been independently exapted in mouse and humans for SMD control of myoblast metabolism. Our work reveals a novel mechanism for the convergent evolution of posttranscriptional gene regulatory networks in mammals by species-specific SINE transposition and SMD.

L.E. Maquat: None. 


\section{S08.3}

Insertion variants at disease risk loci

\section{K. H. Burns}

Johns Hopkins University School of Medicine, Baltimore, MD, United States

Interspersed repeat sequences derived from mobile genetic elements make up much of our DNA. In this presentation, I will introduce the concept that transposable element insertions deliver repressed functions to genomic locations - trans-activating functions bound by repressive silencers, and splicing functions masked by RNA binding proteins. These antithetical properties are the result of complex relationships pitting the intrinsic activities of these retroelements against the host factors that evolve to mitigate their effects. Insertions provide a unique substrate for evolutionary innovation since either of the opposing functions can be made permanent. Our lab studies how the contours of this conflict impact human disease. First, I will describe how specific transposable element loci 'escape' silencing in diseased tissues. For example, long interspersed element-1 (LINE-1) sequences are commonly derepressed in human cancers, resulting in somatic retrotransposition events and potentially creating unique molecular requirements for cancer cell growth. Second, I will describe how rare and commonly-occurring inherited insertion alleles can affect mRNA expression and splicing and lead to genetic disease. Examples include highly penetrant disease alleles, as well as variants with lesser effects that are detectable by genome-wide association study (GWAS). We have cataloged 809 polymorphic Alu elements mapping to 1,159 loci implicated in disease risk by genome-wide association study $($ GWAS) $(\mathrm{P}<10-8)$. We found that $A l u$ insertion variants occur disproportionately at GWAS loci $(\mathrm{P}=0.013)$. Moreover, we have identified many of these Alu elements in linkage disequilibrium (LD) with the trait-associated SNP. We are now working to understand their molecular mechanisms. One variant of interest is an Alu insertion at the CD58 locus on a haplotype that affects multiple sclerosis (MS) susceptibility $(\mathrm{OR}=1.3 \mathrm{p}=3 \times 10-10)$. We found perfect $\mathrm{LD}$ between the trait-associated SNP (rs2300747) and the intronic Alu insertion. We went on to show that the Alu alters splicing of the CD58 mRNA, promoting exon 3 skipping in splice reporter assays, and creating a splice quantitative trait locus (sQTL) in lymphoblastoid cell lines. The aberrant CD58 isoform is frame-shifted, and presumed to be nonfunctional. This work provides a broader perspective on how structural variants of repetitive DNAs may contribute to human disease.

K.H. Burns: None.
S09

Multidimensional nuclear organization

S09.1

Revealing the RNA layer of epigenome

\section{S. Zhong}

University of California San Diego, San Diego, CA, United States

Chromatin-associated RNA (caRNA) could be regarded as a layer of the epigenome (Trends in Genetics, 2018, 34:867-882). To elucidate the RNA layer of epigenome, we developed the iMARGI technology to discover caRNAs and reveal the respective genome interaction loci of each caRNA (Current Biology, 2017, 27(4): 602-609; PNAS 2019, 116 (8) 3328-3337). Applying iMARGI to analyze diverse human cell types, we observed that most of the transcription start sites in the human genome are occupied with long-range interacting caRNAs (Current Biology, 2017, 27(4): 602-609). iMARGI also revealed the nearest chromosomal regions to nuclear speckles (iScience, 2018, 4:204-215) and inter-chromosomal proximity. Furthermore, iMARGI revealed that RNA-DNA interactions in normal cells coincide with fusion RNA pairs in cancers (PNAS 2019, 116 (8) 3328-3337). These data suggest an RNApoise model, where the spatial proximity of one gene's transcripts and the other gene's genomic sequence poises for the creation of fusion transcripts.

S. Zhong: E. Ownership Interest (stock, stock options, patent or other intellectual property); Modest; Genemo Inc. .

\section{S09.2}

\section{The architecture and mechanical properties of the} nuclear lamina

\section{O. Medalia}

University of Zurich, Zurich, Switzerland

Lamins are nuclear intermediate filaments (IFs) of metazoan cells. They assemble into fibrous structures that are positioned between the inner nuclear membrane and the peripheral chromatin, although a small fraction of lamins is present in the nucleoplasm. Lamins are required to maintain nuclear structure and, together with many interaction partners, are involved in most nuclear activities. Mutations in lamins cause a group of $>14$ distinct diseases called laminopathies, it is not clear how lamins are organized in vivo and how these mutations affect lamin functions. Understanding how lamins are assembled, and how mutations in lamins and lamin binding proteins affect lamin 
filament assembly and cellular localization is essential for understanding the basic mechanisms of laminopathic diseases. Here, we apply an integrative approach combining cryo-electron tomography, atomic force microscopy, network analysis, and molecular dynamics simulations to directly measure the structure and mechanical response of single lamin filaments in its three-dimensional meshwork. Individual lamin filaments resolved a globular-decorated fiber appearance and showed that A- and B-type lamins assemble into tetrameric $3.5 \mathrm{~nm}$ thick filaments. The lamin meshwork is not a random arrangement of filaments but the meshwork topology follows 'small world' properties. Our results suggest that the lamin filaments arrange to form a robust, emergent meshwork that dictates the mechanical properties of the lamin filaments.

\section{O. Medalia: None.}

\section{S09.3}

3D genome organisation in disease: patient-specific chromatin interactions from primary tissue

\section{J. M. Vaquerizas}

\section{Max Planck Institute for Molecular Biomedicine, Muenster, Germany}

The three-dimensional organisation of the genome plays a fundamental role in the regulation of gene expression. Recent examinations of chromatin conformation have revealed the presence of hundreds of self-associating domains and thousands of regulatory loops between enhancers and target genes that ensure a correct deployment of developmental programmes. Mutations affecting these highly conserved and relatively tissue-unspecific regulatory features often result in striking developmental defects and disease. Despite the characterisation of such regulatory features, it is currently unknown when chromatin conformation is established, the cellular mechanisms that drive this process and how they are affected in disease.

Here, I will present recent work demonstrating the use of $\mathrm{Hi}-\mathrm{C}$ technologies as a tool for disease characterisation in primary tissue from patients. First, I will introduce an improved Hi-C method for very low amounts of input material, down to one thousand cells. By systematically comparing Hi-C libraries made with decreasing amounts of starting material I will show that the method is highly reproducible and robust to experimental noise. Then, I will show the applicability of this approach in primary B-cells from a large diffuse B-cell lymphoma patient, highlighting patient-specific translocation and abundant local structural variation when compared with control B-cells.

Overall, our results have important implications for our understanding of how the three-dimensional structure of the genome is established and how the mechanisms that trigger this organisation are affected in disease.

J.M. Vaquerizas: None.

\section{S10}

From genome wide association study to mechanisms: fine-mapping

\section{S10.1}

From association to causal variant(s): statistical methods for finemapping

\section{Benner, S. Ripatti, M. Pirinen}

University of Helsinki, Helsinki, Finland

Genome-Wide Association Studies (GWAS) have narrowed down the genome into regions underlying complex phenotypes. However, any one region still harbours thousands of correlated genetic variants, complicating biological follow-up. We therefore need variable selection to refine the large set of variants simply associated with a phenotype down to a much smaller set of putative causal variants with direct effect on the phenotype.

We present the FINEMAP software that couples Bayesian variable selection for fine-mapping causal variants with an ultrafast high-resolution stochastic search. We show that FINEMAP is as accurate as gold-standard exhaustive search implemented in existing fine-mapping approaches when the latter can be completed and achieves even higher accuracy when the latter must be constrained due to computational reasons. We further demonstrate that FINEMAP opens up completely new opportunities by fine-mapping genomic regions with tens of thousands of variants in few minutes while exhaustive search would require thousands of years.

Statistical fine-mapping methods that use GWAS results also require Linkage Disequilibrium (LD) information. We report important practical consequences of misspecification of LD that could happen when publicly available reference genomes are used. We demonstrated both empirically and theoretically that the size of the reference panel needs to scale with the GWAS sample size to produce accurate results. This finding has major implications for the application of all fine-mapping methods using GWAS results from GWAS consortia in which accurate LD information from each participating study is typically not available.

GWAS sample sizes soon counted in millions provide unprecedented opportunities to use fine-mapping results for downstream analysis. Therefore, we investigate how much of the regional heritability can be explained by a finemapping model compared to the variance component model BOLT. Although results from FINEMAP and BOLT show 
good concordance in regions with negligible contribution to the genome-wide heritability, BOLT yields larger estimates than FINEMAP in regions with moderate to high heritability levels. Scaling the analysis to biobank data, we further illustrate how violations of model assumptions on polygenicity induces inaccuracy to heritability estimates that become more accentuated as statistical power to identify causal variants increases.

C. Benner: None. S. Ripatti: None. M. Pirinen: None.

\section{S10.2}

Leveraging genome-wide association studies in diverse populations to fine-map complex human trait loci

\section{A. P. Morris}

\section{University of Manchester, Manchester, United Kingdom}

Genome-wide association studies (GWAS) of complex human traits are increasingly undertaken in diverse populations. One approach that shows great promise for the localisation of causal variants underlying association signals is the aggregation of GWAS across diverse populations through trans-ethnic meta-analysis. Trans-ethnic fine-mapping is enabled by the observation that many lead SNPs for complex traits are shared across diverse populations, which is consistent with a model for which the underlying causal variants are the same across ancestry groups and were derived from mutations that occurred before human population migration out of Africa. Under this model, trans-ethnic fine-mapping then takes advantage of the observation that patterns of linkage disequilibrium (LD) amongst common variants vary between populations. As a result, across ancestry groups, we would expect to see strong association signals only at the causal variant and those SNPs in strong LD with the causal variant in all populations studied: the greater the diversity of GWAS, the more refined the localisation will be. We will review methods for trans-ethnic GWAS meta-analysis, present simulations demonstrating the improved fine-mapping resolution achieved with this approach, and highlight applications of these techniques to localising potential causal variants at complex human trait loci.

A.P. Morris: None.

\section{S10.3}

Large-scale integration of genetic and 'omics' data to find susceptibility genes for obesity and fat distribution

S. L. Pulit ${ }^{\text {, J. Fernandez }}{ }^{2}$, T. Karaderi ${ }^{3}$, S. Laber $^{3}$, T. Brown ${ }^{3}$, A. P. Morris ${ }^{4}$, M. Claussnitzer ${ }^{5}$, C. Lindgren $^{3}$
${ }^{1}$ University Medical Center Utrecht, Utrecht, Netherlands, ${ }^{2}$ Wellcome Trust Center for Human Genetics, Oxford, United Kingdom, ${ }^{3}$ Big Data Institute, Oxford, United Kingdom, ${ }^{4}$ University of Liverpool, Liverpool, United Kingdom, ${ }^{5}$ Broad Institute, Boston, MA, United States

Body fat distribution is a heritable trait and a wellestablished predictor of adverse metabolic outcomes, independent of overall obesity. Recently, we performed a genome-wide association study (GWAS) meta-analysis of 694,649 individuals from the Genetic Investigation of ANthropometric Traits (GIANT) Consortium and UK Biobank (UKBB) and identified 346 genome-wide significant $(\mathrm{p}<5 \times 10-9)$ loci associated to WHR adjusted for BMI (WHRadjBMI). Additionally, we recapitulated the known sexual dimorphism observed in WHRadjBM: 30\% of all loci are sex dimorphic, with most signals showing a larger effect in women. Notably, this sexual dimorphism is virtually absent in GWAS of BMI, prompting a host of questions around the underlying biology of fat distribution and how elucidating (potentially sex-specific) mechanisms could yield insights into the etiology of fat distribution.

We thus set out to perform comprehensive fine-mapping and enrichment testing in the 346 loci associated with WHRadjBMI, as well as the 266 and 91 loci discovered in the women-only and men-only meta-analyses, respectively. We used up to 484,563 samples from the UK Biobank to: (i) examine enrichment of WHRadjBMI-associated loci in hundreds of cell and tissue types, (ii) perform Bayesian finemapping to generate so-called 'credible sets' of likelycausal SNPs, (iii) test for global and local patterns of enrichment of the credible sets in various genomic annotations, and (iv) perform functional work on likely-causal variants and genes.

Global pathway analyses across the full GWAS results replicated the previously-described enrichment of WHRadjBMI signals in subcutaneous $(\mathrm{p}=1.12 \times 10-8)$ and visceral $(\mathrm{p}=1.23 \times 10-6)$ adipose tissue. We additionally observed novel enrichments in reproductive tissues, including cervix and uterus (pectocervix $=5.84 \times 10-5$ and puterus $=8.65 \times 10-7$ ), a notable finding given the link between obesity, fat distribution, and fertility complications.

We next used a Bayesian fine-mapping approach (FINEMAP) to generate credible sets at each locus that capture $99 \%$ of the posterior probability that the causal SNP (s) are in that SNP set (i.e., the '99\% credible set'). We found that for 215 of the $346(\sim 62 \%)$ in the combined sample, FINEMAP identified only 1 likely-causal SNP. A similar proportion of the women-only and men-only loci were most likely to contain only 1 causal SNP.

Finally, we tested to see if our credible sets were enriched in genomic annotations via ENCODE and the Roadmap Consortium. We found that SNPs in credible sets derived 
from the GWAS of WHRadjBMI in men were enriched in ChIP-seq binding tracks for EZH2, a component of the polycomb repressor complex 2 implicated in the adipogenesis pathway. We are currently characterizing the functional effect of SNPs that overlap EZH2 binding motifs.

Our work demonstrates how large-scale GWAS data, in combination with fine-mapping approaches and integration of omics data, can be leveraged to begin revealing perturbations in biological pathways that drive alterations in fat distribution. These efforts may additionally provide insights into sex-specific mechanisms potentially relevant to the treatment of obesity and its sequelae.

S.L. Pulit: None. J. Fernandez: None. T. Karaderi: None. S. Laber: None. T. Brown: None. A.P. Morris: None. M. Claussnitzer: None. C. Lindgren: None.

\section{S11}

\section{De novo developments in epilepsia}

\section{S11.1}

De novo variants in neurodevelopmental disorders with epilepsy

\section{J. Lemke}

Institute of Human Genetics, University of Leipzig, Leipzig, Germany

Neurodevelopmental disorders (NDD) are frequently accompanied by additional features, such as intellectual disability, epilepsy or autism, but little is known about genetic differences between these NDD entities. Investigation of exome-wide de novo variants (DNV) in 6,753 NDD parent-offspring trios revealed unexpected findings when comparing the genetic spectrum of 1,942 individuals with versus 4,811 individuals without epilepsy. Despite marked phenotypic differences, "epileptic encephalopathy" shares a similar genetic spectrum with "NDD with unspecified epilepsy", which is not reflected by current clinical classifications. In general, individuals with missense DNV were more likely to have epilepsy than individuals with truncating DNV. 33 genes displayed a significant excess of DNV in epilepsy cases, of which SNAP25 and GABRB2 had previously only limited evidence of disease association. Joint analysis of the overall cohort also implicated CACNA1E as a novel NDD gene. Furthermore, comparing the designs of 24 diagnostic epilepsy panels of different providers revealed extensive inconsistencies. All panel designs lacked various genes with high diagnostic value and by contrast showed vast excess of genes with low or no diagnostic value. Among genetically solved epilepsy cases, the individual DNV gave rise to personalized treatment approaches in more than $25 \%$, emphasizing the importance and the benefit of accurate genetic diagnostics in NDD, especially when accompanied by epilepsy.

\section{J. Lemke: None.}

\section{S11.2}

\section{Parental Mosaicism in "De Novo" Epileptic Encephalopathies}

\section{H. C. Mefford}

\section{University of Washington, Seattle, WA, United States}

De novo pathogenic variants have been increasingly implicated in severe neurodevelopmental disorders, including developmental and epileptic encephalopathies, over the past several years. Genetic counseling for recurrence risk when a family has a child with a de novo pathogenic variant, estimated at around $1-3 \%$, is largely based on empirical data and accounts for the possibility of germline mosaicism in one parent. In families where parental germline mosaicism is present, however, the true recurrence risk may be as high as $50 \%$. Low-level mosaicism is easily missed by Sanger sequencing technology, but massively parallel sequencing technology permits deep sequencing and allows the detection of mosaic alleles. In this talk, I will discuss work from our group and others to investigate the frequency of parental germline mosaicism for apparently de novo pathogenic variants, which may be higher than previously appreciated. The clinical implications and impact on segregation testing will also be discussed.

\section{H.C. Mefford: None.}

\section{S11.3}

\section{Brain somatic mutations in malformations of cortical development with epilepsy}

\section{S. Baldassari, T. Ribierre, S. Baulac}

\section{Institut du Cerveau et de la Moelle, Paris, France}

Genetic malformations of cortical development, such as focal cortical dysplasia (FCD), are major causes of severe pediatric refractory epilepsies subjected to neurosurgery. Neuropathological hallmarks of FCDs and HMEs include enlarged dysmorphic neurons (DNs) and balloon cells (BCs). Recently, there has been growing evidence that brain somatic variants play a major role in the etiology of these neurodevelopmental disorders. My talk will provide a comprehensive view of the occurrence of germline and somatic variants in a large cohort of patients with FCD and HME. We use ultra-deep sequencing to search for lowallele somatic variants in paired brain/blood patient 
samples, as well as pools of microdissected pathological cells to elucidate the genetic cause. Our study unveils two distinct pathogenic mechanisms involving the non-mTORrelated gene SLC35A2 in FCD1, and mTOR-pathway in FCD2/HME, orienting towards targeted therapies.

We also model somatic mutations in the mouse brain combining in utero electroporation and CRISPR-Cas9 geneediting to reproduce a focal and mosaic genetic hits. Mice with a low-level mosaic rate of crisperized or mutant neurons faithfully reproduced clinical and neuropathological phenotypes of focal epilepsy linked to FCD.

S. Baldassari: None. T. Ribierre: None $\mathbf{S}$. Baulac: None.

\section{S12}

\section{Congenital disorders of glycosylation}

\section{S12.1}

Genetic heterogeneity in CDG: where are the patients?

\section{G. Matthijs}

Center for Human Genetics, University of Leuven, Leuven, Belgium

The field of Congenital Disorders of Glycosylation (CDG) has expanded enormously in 20 years. It has become practically impossible to keep track of their number; a review from last year listed 105 different types (Péanne et al. Eur J Med Genet. 61(11):643-663, 2018). PMM2CDG remains by far the most frequent type 1 CDG (twothirds of the cases collected by the EUROGLYCANET consortium). MAN1B1-CDG and several COG deficiencies are among the more frequent type $2 \mathrm{CDG}$. Almost all CDG are recessive disorders, either autosomal or X-linked. However, the collection of CDG harbours a number of genetically interesting observations. Thus far, ALG1 deficiencies were rarely identified because a large number of pseudogenes complicates diagnostics; this has now been solved by using an NGS approach. COG4-CDG was known as a typical CDG. Recently, it was shown that a recurrent $d e$ novo COG4 mutation causes Saul-Wilson syndrome. Interestingly, a $5^{\prime}$ upstream GGC expansion of the XYLTI gene is present either in a homozygous or in a compound heterozygous state in a large majority of patients with Baratela-Scott syndrome. Defects in the X-linked SLC35A2 gene are dominant, with severe, early onset presentations in girls. De novo mosaics were described in male patients, also severely affected, while somatic mutations in the brain have been shown to cause severe intractable epilepsy. A careful screening of patients with a skeletal dysplasia with amelogenesis imperfecta, yet another CDG phenotype, revealed null alleles in SLC10A7, which have not been explained molecularly. We identified mutations in the $\mathrm{X}$ linked MAGT1 gene in boys. MAGT1 is a homolog of TUSC 3 that was previously associated with intellectual disability. Remarkably, mutations in MAGT1, which is a component of the oligosaccharyltransferase complex, cause both a CDG phenotype and a primary immune deficiency described as XMEN (Blommaert et al. PNAS 116 (20):9865-9870, 2019).

\section{G. Matthijs: None.}

\section{S12.2}

\section{CDG therapies}

\section{E. Morava}

\section{Mayo Clinic, Rochester, MN, United States}

Congenital disorders of glycosylation (CDG) are a group of clinically and genetically heterogeneous metabolic disorders. Over 147 CDG subtypes have been described. CDG types affecting $\mathrm{N}$-glycosylation are the most common type of CDG with emerging therapeutic possibilities. This review summarizes the available therapies for disorders affecting the $\mathrm{N}$-linked glycosylation pathway. In the first part of the review, we highlight the clinical presentation, general principles of management and disease-specific therapies for $\mathrm{N}$-linked glycosylation CDG subtypes, organized by organ system. The second part of the review focuses on the therapeutic strategies currently available and under development. We summarize the successful (pre-) clinical application of nutritional therapies, transplantation, activated sugars, gene therapy and pharmacological chaperones and outline the anticipated expansion of the therapeutic possibilities in CDG. We aim to provide a comprehensive update on the treatable aspects of CDG types involving $\mathrm{N}$-linked glycosylation, with particular emphasis on disease-specific treatment options for the involved organ systems and present current and future therapeutic strategies for CDG.

\section{E. Morava: None.}

\section{S12.3}

\section{Link between Golgi ion homeostasis defects and Congenital Disorders of Glycosylation}

\section{F. Foulquier}

CNRS UMR 8576, University of Lille, Villeneuve D'Ascq, France

Within the secretory pathway, ionic homeostasis of each organelle is unique in terms of $\mathrm{H}^{+}, \mathrm{Ca}^{2+}$ and $\mathrm{Mn}^{2+}$ 
concentrations. Many transporters and pumps finely regulate these homeostasis in the Golgi compartment, known to be crucial for many cellular processes such as the vesicular fusion event, the secretion of proteins but also the activities of Golgi glycosyltransferases and glycosidases. For example, in eukaryotic cells, the $\mathrm{Ca}^{2+}$ concentration along the secretory pathway (ER/ Golgi) decreases progressively. Many pumps and transporters are involved in this tight regulation such as SERCA and SPCA1 pumps. Mutations of the secretory pathway $\mathrm{Ca}^{2+}$-ATPase isoform 1 (SPCA1) cause a rare autosomal-dominant skin disorder called Hailey- Hailey disease. Similarly, mutations of ER SERCA $\mathrm{Ca}^{2+}$-ATPase cause also a rare autosomal-dominant skin disorder called Darier Disease. Alterations of Golgi pH can also cause diseases such as autosomal Cutis laxa (type II) where $\mathrm{N}$ - and O-glycosylation are altered as well as the trafficking of specific proteins. To add an extra layer of complexity, defects in TMEM165, SLC39A8 and recently in SLC10A7, were discovered to be associated to strong Golgi glycosylation abnormalities leading to Congenital Disorders of Glycosylation (CDG). In these deficiencies, disruptions in the Golgi $\mathrm{Ca}^{2+} / \mathrm{Mn}^{2+}$ homeostasis are thought to be the molecular cause leading to glycosylation defects. This talk will first summarize the molecular machineries involved in the ER/ Golgi ion homeostasis regulation and then the associated diseases resulting from defects in Golgi ion homeostasis.

F. Foulquier: None.

\section{S13}

\section{Understanding mutations to detect cancer}

\section{S13.1}

\section{Understanding mutational processes and tumor biology}

\section{A. Gonzalez-Perez ${ }^{1,2}$}

${ }^{1}$ Institute for Research in Biomedicine, The Barcelona Institute of Science and Technology, Barcelona, Spain, ${ }^{2}$ Department of Experimental and Health Sciences, University Pompeu Fabra, Barcelona, Spain

In our lab, we use data on genomic mutations in tumors in two broad areas of research. On the one hand, we study the distribution of mutations across different regions in the genome to understand basic questions about molecular biology, such as the interplay between DNA damage and repair, and other cellular processes. With these studies we have discovered different small-scale genomic features -i.e., spanning between a dozen and a few hundred base pairsthat interfere with the deposition of different types of DNA damage and the mechanisms of DNA repair. For example, we recently showed that the periodicity in the orientation of the DNA minor groove with respect to nucleosomes determine a periodic pattern in the generation of somatic mutations across human tumors, germline variants across populations, and genetic divergence across closely related species. We demonstrated that the known widespread WW periodicity across eukaryotic genomes $-10-\mathrm{bp} \mathrm{A} / \mathrm{T}$ dinucleotides periodicity- could have arisen as a result of the periodic de novo mutation rate.

On the other hand, we study the genetic basis of tumors and their basic biology. Combining an ensemble of bioinformatics tools developed in our group and others with the aim of detecting signals of positive selection in the pattern of mutations of genes across tumors, we have produced a catalog of the genes that drive tumorigenesis in 60 cancer types. We go one further step to identify which amongst all the mutations of these genes drive each individual tumor. I will present IntOGen, a cancer genomics platform that makes available to all researchers the catalog of driver genes and driver mutations across more than 50,000 tumors. Different types of somatic alterations affect both oncogenes and tumor suppressors. Recently, we have carried out the first systematic analysis of the role of abnormal protein degradation in tumorigenesis. We found that more than $10 \%$ of all driver mutations across well-known cancer genes cause tumorigenesis through the abnormal stabilization of oncoproteins.

\section{A. Gonzalez-Perez: None.}

\section{S13.2}

Finding a germline mutation during tumor testing: implications for the patient and the family

\section{J. N. Weitzel}

City of Hope Comprehensive Cancer Center, Los Angeles, CA, United States

Precision medicine and the genomic sequencing of tumors are increasingly well suited to targeting cancer therapies. In addition, germline variants such as $B R C A$ have emerged as companion diagnostics for targeting PARP inhibitors. Technically, a tumor genome is the germline with a few added features. This presentation focuses on this intersection of tumor and germline, wherein we consider the discovery of germline mutations in precision medicine, and the implications for the patient and the family. While appreciating the importance of germline variants in treatment response in precision medicine, we will discuss critical issues in genomic cancer risk assessment and cancer prevention, with a focus on hereditary cancer, such as the process for confirmation of potential germline variants 
discovered in tumor or cell free DNA testing, and cascade testing for families.

J.N. Weitzel: None.

\section{S13.3}

\section{Liquid biopsy to follow clonal evolution in cancer}

B. Mussolin ${ }^{1}$, G. Siravegna ${ }^{2,1}$, L. Lazzari ${ }^{1,3,2}$, G. Crisafullit, ${ }^{1,2}$ A. Cassingena ${ }^{4}$, G. Rospo ${ }^{1}$, M. Montone ${ }^{1}$, G. Corti ${ }^{1}$, A. Bartolini ${ }^{1}$, R. B. Lanman ${ }^{5}$, C. Martino ${ }^{1}$, F. Loupakis ${ }^{6}$, D. Regge ${ }^{1}$, F. Di Nicolantonio ${ }^{1,2}$, A. Sartore-Bianchi ${ }^{4}$, A. Bertotti, ${ }^{1,2}$, L. Trusolino ${ }^{1,2}$, S. Siena ${ }^{4}$ S. Marsoni ${ }^{1,3,4}$, A. Bardelli ${ }^{2,1}$

${ }^{1}$ Candiolo Cancer Institute - Fondazione Piemontese per l'Oncologia (FPO), Istituto di Ricovero e Cura a Carattere Scientifico (IRCCS), Candiolo, Italy, ${ }^{2}$ University of Torino, Department of Oncology, Torino, Italy, ${ }^{3}$ FIRC Institute of Molecular Oncology (IFOM), Milano, Italy, ${ }^{4}$ Niguarda Cancer Center, Ospedale Niguarda Ca' Granda,, Milano, Italy, ${ }^{5}$ Guardant Health, Inc.,, Redwood City, CA, United States, ${ }^{6}$ Department of Oncology, Veneto Institute of Oncology IOV - IRCCS, Padova, Italy

Tumors evolve by a reiterative process of genetic diversification and clonal evolution. Genotyping cancer tissues has inherent limitations and tissue biopsies cannot be performed repeatedly. Liquid biopsy-tumor genotyping using circulating cell-free tumor DNA (ctDNA) in the blood- has been recently applied to molecularly profile cancer patients, to monitor tumor burden during therapy and to uncover novel mechanisms of resistance to targeted therapies. We used metastatic colorectal cancer (mCRC) as a model to assess whether liquid biopsies could in principle uncover molecular mechanisms responsible for the emergence of resistance and track clonal evolution during targeted therapies. Emerging evidence indicates that escape from EGFR and HER2 targeted therapy are molecularly heterogeneous and involve several genes. We exploited plasma ctDNA analysis by Next Generation Sequencing and Digital PCR to define gene alterations responsible for primary or acquired resistance to anti-EGFR and anti HER2 therapies. While most $\mathrm{mCRC}$ patients who receive antiEGFR antibodies develop KRAS mutations during treatment, we found that additional mutations affecting key nodes of the EGFR signaling pathway can also confer resistance. Mutated KRAS clones, which emerge in blood during EGFR blockade, decline upon withdrawal of EGFRspecific antibodies, indicating that clonal evolution continues beyond clinical progression. ctDNA profiles of individuals who benefit from multiple challenges with anti-EGFR antibodies exhibit pulsatile levels of mutant $K R A S$. These results indicate that the CRC dynamically adapts to intermittent drug schedules and provide a molecular explanation for the efficacy of rechallenge therapies based on EGFR blockade. In parallel, we focused on HER2 amplified CRC. Targeting HER2 is effective in $30 \%$ of ERBB2-amplified mCRC, and most sensitive cases eventually relapse. We studied the molecular evolution of individual metastases during HER2 targeted treatment to discover spatially resolved determinants of resistance in mCRC. ctDNA analysis identified alterations associated with lack of response in the majority of refractory patients. CtDNA profiles and lesion-specific radiographic reports revealed organ- and metastasis-private evolutionary patterns in patients who had initially responded. Of clinical relevance, we found that a simple blood assay would have identified the HER2 sensitive patients. Finally, exploiting whole exome data from individual lesions we designed metastases-specific SNV panels. When the latter were coupled with T-Cell Receptors profiles (corresponding to metastases-specific T-Cells infiltrates) we were able to concomitantly track in the blood the evolution of neoplastic and T-Cells during several rounds of therapy. Blood-based analyses also revealed a direct correlation between lesions' size and contribution to plasma ctDNA pool, an information which was previously not available in metastatic colorectal cancers.

B. Mussolin: None. G. Siravegna: None. L. Lazzari: None. G. Crisafulli: None. A. Cassingena: None. G. Rospo: None. M. Montone: None. G. Corti: None. A. Bartolini: None. R.B. Lanman: A. Employment (full or part-time); Significant; Guardant Health, Inc., Redwood City, California.. E. Ownership Interest (stock, stock options, patent or other intellectual property); Significant; Guardant Health, Inc., Redwood City, California.. C. Martino: None. F. Loupakis: None. D. Regge: None. F. Di Nicolantonio: None. A. Sartore-Bianchi: None. A. Bertotti: None. L. Trusolino: None. S. Siena: None. S. Marsoni: None. A. Bardelli: None.

\section{S14}

Debate: Genomics and the Media

\section{S14.2}

Genetics and Social Media

\section{E. T. Matloff}

\section{My Gene Counsel, New Haven, CT, United States}

Public perception of science and medicine plays an important role in how well therapies and medical advances are integrated into society, used and accepted by ordinary citizens, funded by investors and the government, and 
therefore embraced or halted. One striking example is pediatric vaccinations. Despite decades' worth of scientific evidence demonstrating the safety and efficacy of vaccines, recent public dialogue has created a surge in uncertainty, distrust, and fear significant enough to reshape acceptance and use of vaccines in many areas, and to trigger outbreaks of diseases that were once thought obsolete. This is because public perception is based not only on scientific data and medical knowledge, but on narratives shared on social media platforms and opinions expressed by outspoken critics and celebrities, many of whom have no medical training, experience, or knowledge.

For these reasons, scientists and clinicians should care about media coverage of science and medicine and should help shape the messaging to ensure the information that reaches the public is accurate, easy-to-understand, and can be used effectively. When knowledgeable professionals are not involved in these dialogues, the answers fed to the public are often inaccurate and can be damaging to the field and to individual consumers. This is particularly true in genomics, where technology and implementation of genetic testing is evolving rapidly. Geneticists need to take a more active role in helping to educate the public and the media. This includes not only interaction with the mainstream media and popular press, but also direct engagement on social media platforms, such as Facebook, Twitter, Instagram, and Reddit. The investment of time and resources necessary to educate the public and to shape the conversation about genetics and genomics is a worthwhile and necessary effort for students, clinicians, scientists, educators, and leaders in our field.

E.T. Matloff: A. Employment (full or part-time); Significant; My Gene Counsel, LLC.

\section{S14.3}

\section{Human germline genome editing: The public and the pundits}

\section{F. Baylis}

\section{Dalhousie University, Halifax, NS, Canada}

In the Spring of 2015, Junjiu Huang and his colleagues at Sun Yat-sen University in Guangzhou China published their research describing the first-in-human use of CRISPR genome editing technology in early stage, nonviable embryos. The research objective was to correct a mutation in the HBB gene that causes $\beta$-thalassemia. This controversial research lead to the first International Summit On Human Gene Editing in December 2015. At the close of the Summit, the Organizing Committee issued a statement in which they decreed that "it would be irresponsible to proceed with any clinical use of germline editing unless and until the safety issues have been dealt with and there is broad societal consensus."

The 2015 Summit statement did not use the word moratorium. Nonetheless, it was widely reported as a call for a moratorium. At the time, everyone agreed that no one should proceed with heritable human genome editing.

Three years later, in November 2018, Chinese scientists again took the world by storm. Jiankui $\mathrm{He}$, a researcher at the Southern University of Science and Technology in Shenzhen China, announced the birth of twin girls with edited genomes. The research objective in this instance was to provide the children with resistance to HIV by modifying the CCR5 gene. This announcement occurred on the eve of the Second International Summit On Human Genome Editing in November 2018. At the close of this Summit, the Organizing Committee issued a statement both condemning the research conducted by $\mathrm{He}$ and calling for a "translational pathway to germline editing." There was no renewed call for "broad societal consensus" and no statement that could be interpreted as a call for a moratorium.

This presentation will critically examine the media coverage surrounding each of these events as well as the twitter conversations. Who said what, when and why ...

F. Baylis: None.

\section{S15}

\section{Regulatory Landscapes}

\section{S15.2}

Regulation of disease-associated gene expression in the 3D genome

\section{W. De Laat, P. Krijger, M. Verstegen, G. Geeven, V. Bianchi, C. Vermeulen, N. Rinzema, E. Vos, A. Allahyar, C. Valdes, M. Pieterse, M. Sharma}

\section{Oncode \& Hubrecht Institute, Utrecht, Netherlands}

Genomic organization in space and time (4D genome) has regulatory impact on gene expression and the epigenetic landscape. We develop new methods to understand chromatin topology in high detail, from the single allele to the cell population level, to assign function to the noncoding genome in health and disease. Latest technologies and biological insights will be presented. Additionally, we develop and will present new strategies to detect translocations in FFPE tumor samples.

W. De Laat: E. Ownership Interest (stock, stock options, patent or other intellectual property); Modest; Cergentis. P. Krijger: None. M. Verstegen: None. G. Geeven: None. V. Bianchi: None. C. Vermeulen: None. N. Rinzema: None. 
E. Vos: None. A. Allahyar: None. C. Valdes: None. M. Pieterse: None. M. Sharma: None.

\section{S15.3}

Identifying DNA-regulatory elements in non-traditional model systems

\section{Garfield}

\section{IRI Life Sciences/Humboldt University, Berlin, Germany}

Many of the mutations that define us - the ones that make us tall vs. short or healthy vs. sick and even the mutations that make us human, are found distributed across the noncoding, regulatory genome. Identifying and characterizing these mutations, however is challenging; unlike coding loci, the locations and function of DNA regulatory elements cannot typically be determined by DNA sequence alone. Regulatory elements are also highly context specific, with many elements showing activity and accessibility to protein binding in a subset of tissues or developmental stages. Single-cell chromatin profiling provides on solution to these issues by allowing researchers to identify protein-bound regulatory elements across multiple tissues and developmental stages in a single experiment.

In this talk, I will present an overview of how my lab is using single-cell chromatin profiling in non-mammalian model systems (Drosophila and sea urchins) to better understand the regulatory elements active during embryogenesis and how mutations impact their function. I will focus specifically on two questions: 1) How do tissue/stagespecific regulatory architectures influence the functional consequences of mutations? 2) What are regulatory elements specified during the earliest stages of embryogenesis?

D. Garfield: None.

\section{S16}

\section{Methods for genetic epidemiology}

\section{S16.3}

Large-scale inference of human genetic data

\section{A. Rivas}

\section{Stanford University, Stanford, CA, United States}

Instead of racing to cure disease after the fact, we hope to prevent disease before it strikes. In order to realize the full potential of Precision Health we can learn the opportunities by integrating massive-scale datasets and advanced computational algorithms. In this talk, I will present the resources and statistical learning approaches we have developed in
Rivas Lab using population scale genomic data and medical records in approximately 500,000 individuals from the UK Biobank study. The algorithms and computational tools are designed to learn about disease risk attributed to genes, to improve learning across populations, and to provide precise characterization of genetic risk across diseases for an individual.

M.A. Rivas: None.

\section{S17}

ESHG-ASHG Debate: Global collaboration to advance the use of genomics in health

S17.1

Challenges and solutions to advance global

collaboration to understand, diagnose, and develop therapies for rare diseases

\section{K. M. Boycott}

Children's Hospital of Eastern Ontario, University of Ottawa, Ottawa, ON, Canada

Rare diseases, which affect over 350 million people worldwide, frequently go undiagnosed or misdiagnosed for years, in large part because access to genetic testing across the world is far from optimal and because the genetic cause of at least one-third of rare diseases has yet to be discovered. And while investment in the development of rare disease therapies has been encouraged by the introduction of regulations, policies and incentives, there are treatments for only $6 \%$ of rare diseases, of which fewer than $1 \%$ are curative. A vast, unmet need for research and investment to advance diagnostic capabilities and therapeutic development must be confronted, despite the myriad of challenges faced. Collaborative strategies that pool resources and knowledge are vital, including team science, research networks, novel funding models, shared knowledge platforms, and innovative regulatory frameworks. The International Rare Diseases Research Consortium (IRDiRC), established in 2011, has been working diligently to unify stakeholders (e.g., funding bodies, companies, umbrella patient advocacy groups, researchers, and experts) to seek and drive solutions that aim to accelerate diagnosis and therapeutic development for rare diseases worldwide. Further and future advances will depend on continued collaborations and cooperation among stakeholders, working hand in hand with patients, and exponentially improving research and development efficiency. Critically, engagement with stakeholders from underrepresented populations and less developed countries must be prioritized, to enable 
all people living with a rare disease to receive an accurate diagnosis, care, and therapy.

K.M. Boycott: None.

\section{S17.2}

\section{EMBL-EBI and global data integration}

\section{N. Goldman}

EMBL-European Bioinformatics Institute, Hinxton, United Kingdom

Molecular biology is now a leading example of a data intensive science, with both pragmatic and theoretical challenges being raised by data volumes and dimensionality of the data. These changes are present in both "large scale" consortia science and small scale science, and across now a broad range of applications - from human health, through to agriculture and ecosystems. All of molecular life science is feeling this effect.

Molecular techniques - from genomics through transcriptomics and metabolomics - continue to drop in price and turnaround time. This creates a wealth of opportunity for clinical research and, in some cases, active changes in clinical practice even at this early stage. The development of this work requires inter-disciplinary teams spanning basic research, bioinformatics and clinical expertise.

This shift in modality is creating a wealth of new opportunities and has some accompanying challenges. In particular there is a continued need for a robust information infrastructure for molecular biology and clinical research. This ranges from the physical aspects of dealing with data volume through to the more statistically challenging aspects of interpreting it.

A particular opportunity is the switch from research commissioning genomic measurement to healthcare-centric genomic measurement. This is occurring in a number of countries worldwide, including Australia, Denmark, Finland, France, United Kingdom and United States. The Global Alliance for Genomics and Health is providing a standards-setting organisation to allow for both a deepening of the technical aspects of healthcare and for appropriate secondary use for research of healthcare-commissioned genomics data.

I will outline the overall challenge present in this new, interdisciplinary field, the global coordination needed to achieve its goals, and the role that the EMBL-European Bioinformatics Institute is seeking to play.

N. Goldman: None.

\section{S17.4}

Pulling the Strands Together: MEGA Steps to Drive European Genomics and Personalised Medicine

\section{D. Horgan}

European Alliance for Personalised Medicine, Bruxelles, Belgium

The increasing understanding of the genome is recognised as being one of the main determinants of future improvement in healthcare. The availability of genetic data from a large number of individuals increases the ability to investigate questions across many rare and common diseases and in different populations, and also provides more information for understanding clinical care outcomes for an individual. A number of large scale genome sequencing initiatives have been launched in the last few years to try and capitalise on this potential. Within Europe, the UK has led the way with the 100,000 Genomes Project. This project looks at the genome sequences of patients with rare diseases or cancer. More recently France announced plans to invest EUR 670 million in a genomics and personalised medicine programme. In the US, the Precision Medicine Initiative aims at large-scale research by gathering one million or more volunteers to extend precision medicine to all diseases. Meanwhile, China has announced plans to invest nearly USD 10 billion in its own precision medicine initiative. These projects demonstrate the commitment at a national level and raise the question "What benefits would be realised by undertaking a million genome initiative in a coordinated effort across European countries?" A coordinated, pan-European MEGA project would garner crucial genetic information that could have an immeasurable benefit when it comes to the health of current and future EU citizens.

D.D. Horgan: None.

\section{S18}

Our genetic history and its phenotypic consequences

\section{S18.1}

Tales of Early Humans, Admixture, and Adaptation

\section{Jakobsson}

Uppsala University, Uppsala, Sweden

Advances in the sequencing and the analysis of the genomes of both modern and ancient individuals have led to breakthroughs in understanding human evolutionary history. As an example, I will discuss recent discoveries about human history in Africa, leading to a fundamental revision of the age of our species. Recent sequencing of seven ancient individuals from KwaZulu-Natal, South Africa, of which three were dated to $\sim 2000$ years old, 
provided direct evidence for migration, admixture and adaptation in southern Africa that had important consequences for interpreting our human past. Using traditional and new population genomics analysis approaches, we estimate the deepest human population divergence time to between 350,000 and 260,000 years ago. This estimate increases the deepest divergence among modern humans, coinciding with anatomical developments of archaic humans into modern humans as represented in the local fossil record, and suggest that modern humans emerged around 300,000 years ago. As we uncover new pieces to the human evolution puzzle, a complex interplay between migration, admixture and adaptation often appear.

M. Jakobsson: None.

\section{S18.2}

Timing past admixture events and characterizing their consequences in contemporary human populations

\section{G. Hellenthal, L. Ormond, P. Wangkumhang}

\section{University College London, London, United Kingdom}

Periods of intermixing among populations, or admixture events, have had a major influence in shaping genetic diversity, sometimes with consequences for particular genetics-based traits. Here I present a new approach (called fastGLOBETROTTER) to identify and date admixture using dense genome-wide Single-NucleotidePolymorphism (SNP) data that is more accurate and 4-20 fold faster than the GLOBETROTTER software that similarly exploits haplotype information to increase precision over existing approaches. I demonstrate the upper limit of time depths on historic intermixing that these techniques can detect reliably when using increasingly larger cohorts of genetically similar individuals. Via application to data from over 6000 Europeans, I report new results on admixture signals in northwest Europe, including events dated to $\sim 500-600 \mathrm{CE}$ from sources carrying DNA related to Africans found in individuals within Belgium, France and certain parts of Germany.

Furthermore, I illustrate the phenotypic effects that recent migration has had on a geographic region, such as height in the United Kingdom. I describe new statistical approaches that can model admixture processes when identifying genetic regions affected by selection. These techniques can (1) identify the loci and populations involved in selection, (2) enable the joint testing of SNP sets (e.g. in pathways), and (3) account for variation in data quality when incorporating DNA from ancient human remains. Testing for selection using a variety of world-wide human populations and DNA from both ancient and modern humans, I report new findings on selection attributable to infection and metabolism, including insights into the time periods over which this selection has been acting.

G. Hellenthal: Other; Modest; Founding member of GenSci. L. Ormond: None. P. Wangkumhang: None.

\section{S18.3}

Consequences of population genetic differences in genetic risk prediction across diverse human populations

\section{A. R. Martin 1,2,3, M. Kanai ${ }^{4,1,2,3,5}$, Y. Kamatani ${ }^{5,6}$, Y. Okada ${ }^{5,7,8}$, B. M. Neale ${ }^{1,2,3}$, M. J. Daly ${ }^{1,2,3,9}$}

${ }^{1}$ Analytic and Translational Genetics Unit, Massachusetts General Hospital, Boston, MA, United States, ${ }^{2}$ Program in Medical and Population Genetics, Broad Institute of Harvard and MIT, Cambridge, MA, United States, ${ }^{3}$ Stanley Center for Psychiatric Research, Broad Institute of Harvard and MIT, Cambridge, MA, United States, ${ }^{4}$ Department of Biomedical Informatics, Harvard Medical School, Boston, MA, United States, ${ }^{5}$ Laboratory for Statistical Analysis, RIKEN Center for Integrative Medical Sciences, Yokohama, Japan, ${ }^{6}$ Kyoto-McGill International Collaborative School in Genomic Medicine, Kyoto University Graduate School of Medicine, Kyoto, Japan, ${ }^{7}$ Department of Statistical Genetics, Osaka University Graduate School of Medicine, Suita, Japan, ${ }^{8}$ Laboratory of Statistical Immunology, Immunology Frontier Research Center (WPI-IFReC), Osaka University, Suita, Japan, ${ }^{9}$ Institute for Molecular Medicine Finland (FIMM), University of Helsinki, Helsinki, Finland

Polygenic risk scores (PRS) are poised to improve biomedical outcomes via precision medicine. However, the major ethical and scientific challenge surrounding clinical implementation of PRS is that those available today are several times more accurate in individuals of European ancestry than other ancestries. This disparity is an inescapable consequence of Eurocentric biases in genomewide association studies, thus highlighting that--unlike clinical biomarkers and prescription drugs, which may individually work better in some populations but do not ubiquitously perform far better in European populations-clinical uses of PRS today would systematically afford greater improvement for European-descent populations. Early diversifying efforts show promise in leveling this vast imbalance, even when non-European sample sizes are considerably smaller than the largest studies to date. To realize the full and equitable potential of PRS, greater diversity must be prioritized in genetic studies, and summary statistics must be publicly disseminated to ensure that health disparities are not increased for those individuals already most underserved. 
A.R. Martin: None. M. Kanai: None. Y. Kamatani: None. Y. Okada: None. B.M. Neale: None. M.J. Daly: None.

\section{S19}

\section{Treating rare genetic disease}

\section{S19.2}

\section{Gene therapy for inherited neuromuscular disorders}

\section{F. Muntoni ${ }^{1,2}$ \\ ${ }^{1}$ University College London Great Ormond Street Institute of Child Health, London, United Kingdom, ${ }^{2}$ University College Great Ormond Street Institute Child Health, London, United Kingdom}

Childhood neuromuscular disorders are devastating conditions associated with major disability and shortened life span. They encompass common muscular dystrophies such as Duchenne muscular dystrophy (DMD); motorneuron diseases such as spinal muscular atrophy (SMA), and less common limb girdle muscular dystrophies, peripheral neuropathies and congenital myopathies. Each of these conditions presents its own therapeutic challenges. In muscular dystrophies one major obstacle is the abundance of the target organ (skeletal muscle is $\sim 40 \%$ of the body weight), the need to target the heart and, as far as DMD is concerned, the size of its cDNA that, at $14 \mathrm{~Kb}$, precludes the use of commonly used viral vectors. For SMA, one of the challenges is the need to target all motorneurons, and the short window for therapeutic intervention in this rapidly progressive condition affecting infants. For congenital myopathies such as myotubular myopathy, the profound severity of affected children and their fragility complicate the design and execution of clinical trials. The landscape for these conditions is however very changing rapidly, thank to early success in landmark studies, of which the most advanced are those in SMA and DMD. An AAV9 vector originally developed at Columbus University, has demonstrated to be able to efficiently correct motorneurons after intravenous administration, with acquisition of unprecedented functional achievements in SMA1 patients. The same group in Columbus also initiated in 2018 the first AAV9 clinical trial for DMD, in which a highly internally deleted "minidystrophin" is packaged. Early data appear encouraging. Shortly after 2 additional industry sponsored studies initiated their AAV9 DMD clinical trials. Recent data also indicate that children affected by the severe myotubular myopathy, in whom the defective gene was administered after AAV8 i.v. injection have obtained benefit from the treatment. In my presentation I will summarise recent results, and future challenges

F. Muntoni: B. Research Grant (principal investigator, collaborator or consultant and pending grants as well as grants already received); Modest; Sarepta, Biogen, Avexis. F. Consultant/Advisory Board; Modest; Pfizer, Roche.

\section{S19.3}

\section{Gene therapy for hemoglobinopathies}

\section{G. Ferrari}

San Raffaele Telethon Institute for Gene Therapy (SRTIGET), Milan, Italy

Sickle cell disease (SCD) and ß-thalassemia major are congenital anemias caused by mutations in the B-globin gene, resulting in either abnormal hemoglobin structure or reduced/absent production of B-globin chains. They are the most common monogenic disorders associated to morbidity and mortality. Treatment of these diseases is essentially supportive, requiring transfusions, iron chelation and use of hydroxycarbamide in SCD. At present, the only curative approach is represented by allogeneic hematopoietic stem cell transplantation, with a probability to find a wellmatched donor of $<25 \%$.

Ex vivo gene therapy, using autologous genetically modified hematopoietic stem cells, potentially represents a cure applicable to all patients regardless of donor availability and free from transplant related immunological complications such as graft rejection and graft-versus-hostdisease. The development and large scale production of clinical grade HIV-derived lentiviral vectors expressing human B-globin, and the optimization of gene transfer protocols in HSCs have progressed this field to the pioneering clinical trials in France and in U.S.A., and more recently in Italy. The first results of clinical benefit, including early engraftment, hemoglobin expression and transfusion independence were reported for some patients and are proving the potential efficacy of this therapeutic approach.

Our contribution to the field of gene therapy in the last 10 years was devoted to the clinical development of a safe gene therapy approach for ß-thalassemia using the GLOBE lentiviral vector. The crucial steps leading to TIGET BTHAL clinical trial, as well as preliminary data on treated patients, will be presented.

G. Ferrari: None.

\section{S20}

\section{Epigenetics and early development}




\section{S20.1}

\section{Single Cell Epigenomic Analysis of the Anatomy and Neuronal Circuitry of the Brain}

\section{J. R. Ecker ${ }^{1,2}$}

${ }^{1}$ Howard Hughes Medical Institute, La Jolla, CA, United States, ${ }^{2}$ Howard Hughes Medical Institute \& The Salk Institute for Biological Studies, La Jolla, CA, United States

Our group uses advanced technologies in single cell genomics and neuronal tracing to identify both the molecular signatures of individual neuronal cell types in the mammalian brain and their synaptic partners. We use a variety of multi-omic assays including DNA methylation, RNA-seq, chromatin accessibility and 3D structure in single neurons across the brain. These detailed cell atlases reveal novel cellular taxonomy in the brain and elucidate spatial diversification in fine cell subtypes allowing the identification of cell type-specific DNA regulatory elements. From this information, we can infer transcriptional regulatory networks underlying cell diversification. To link these epigenomically defined cell types to the mouse connectome, AAV2retro has been used to label, isolate, and generate single nuclei methylome data from individual projection neurons for more than 50 neuronal pathways. Our analyses show that the laminar location, cortical region, and projection targets all contribute to the unique epigenetic signatures of individual neurons.

J.R. Ecker: F. Consultant/Advisory Board; Modest; Zymo, Inc, Cibus, Inc.

\section{S20.2}

Genetic-epigenetic interactions: mechanistic insights and practical applications

\section{B. Tycko}

HMH Center for Discovery and Innovation, Nutley, NJ, United States

Our lab, with our collaborators, has been working to understand genetic-epigenetic interactions that occur in cis and in trans. Haplotype-dependent allele-specific DNA methylation (hap-ASM) is a pervasive phenomenon that reflects cis-acting effects of local DNA sequences on local $\mathrm{CpG}$ methylation patterns in human cells and tissues. Differential DNA methylation in Down syndrome (DS-DM) is an interesting trans-acting effect, in which the presence of the extra chromosome 21 leads to recurrent changes in $\mathrm{CpG}$ methylation patterns at loci distributed throughout the genome. I will briefly cover both topics, emphasizing the practical utility of hap-ASM mapping as a post-GWAS approach for homing-in on mechanistically informative disease-associated regulatory sequence variants, and the analysis of DS-DM to test mechanistic hypotheses for how DNA methylation patterns are shaped in mammalian genomes.

References

1: Do C, Shearer A, Suzuki M, Terry MB, Gelernter J, Greally JM, Tycko B. Genetic-epigenetic interactions in cis: a major focus in the post-GWAS era. Genome Biol. 2017 Jun 19;18(1):120

2: Do C, Xing Z, Yu YE, Tycko B. Trans-acting epigenetic effects of chromosomal aneuploidies: lessons from Down syndrome and mouse models. Epigenomics. 2017Feb;9(2):189-207

3: Do C, Lang CF, Lin J, Darbary H, Krupska I, Gaba A, Petukhova L, Vonsattel JP, Gallagher MP, Goland RS, Clynes RA, Dwork A, Kral JG, Monk C, Christiano AM, Tycko B. Mechanisms and Disease Associations of Haplotype-Dependent Allele-Specific DNA Methylation. Am J Hum Genet. 2016 May 5;98(5):934-955.

4: Mendioroz M, Do C, Jiang X, Liu C, Darbary HK, Lang CF, Lin J, Thomas A, Abu-Amero S, Stanier P, Temkin A, Yale A, Liu MM, Li Y, Salas M, Kerkel K, Capone G, Silverman W, Yu YE, Moore G, Wegiel J, Tycko B. Trans effects of chromosome aneuploidies on DNA methylation patterns in human Down syndrome and mouse models. Genome Biol. 2015 Nov 25;16:263.

B. Tycko: None.

\section{Educational Sessions}

E01

New technologies (Sponsored by Illumina)

\section{E01.2}

Resolving human genetic variation with long-read single-molecule sequencing

\section{J. P. Chaisson}

University of Southern California, Los Angeles, CA, United States

As whole genome sequencing is being applied to large disease studies, researchers are faced with the challenge of detecting a wide range of variation, including large insertions, deletions, and rearrangements called structural variation (SV) with short read high-throughput sequencing (HTS) data. It is common use the consensus of multiple algorithms to detect SV with minimal false discovery, 
however the extent of missing variation is unknown. To address this, we applied a suite of approaches: long-read, short-read, strand-specific sequencing technologies, optical mapping, and variant discovery algorithms to comprehensively analyze three human parent-child trios to define the full spectrum of human genetic variation in a haplotyperesolved manner. We identify 818,054 indel variants $(<50$ bp) and 27,622 SVs ( $\geq 50 \mathrm{bp}$ ) per human genome. We also discover 156 inversions per genome-most of which previously escaped detection. Fifty-eight of the inversions we discovered intersect with the critical regions of recurrent microdeletion and microduplication syndromes. Taken together, our SV callsets represent a sevenfold increase in SV detection compared to most standard HTS studies, including those from the 1000 Genomes Project. We use these SV callsets to assess the performance of HTS studies including the types of variation that are missed by many current studies, SV detected in functional noncoding DNA, and the combinations of algorithms that most effectively detect SV in HTS reads.

\section{M.J.P. Chaisson: None.}

\section{E02}

\section{Epigenetics and cancer}

\section{E02.1}

\section{Cancer Epigenetics: from DNA to RNA modifications}

\section{F. Fuks}

\section{University of Brussels, Brussels, Belgium}

DNA modifications and histone post-translational modifications are considered the two pillars of epigenetics. Indeed, cells can chemically modify DNA to dynamically modulate gene transcription and this phenomenon, together with histone protein modification, is known as epigenetic regulation of gene expression. It is regulated by a variety of factors, including developmental cues and environmental stimuli, and alterations in epigenetic control play essential roles in several genetic diseases.

We will address the following key issues in the field of epigenetics and epigenomics:

1. Since DNA methylation is a reversible process, there is potentially a huge benefit in modulating DNA methylation for the treatment of human diseases, notably cancer. To achieve such anti-cancer therapy, it is essential to decipher the underlying molecular mechanisms. An overview of the most recent mechanisms will be presented.

2. We will also describe recent epigenomic efforts to better understand and map epigenetic alterations in cancers, including DNA methylation and hydroxymethylation.
3. Although the multiple layers of epigenetic regulation that result from modifications of DNA and histones have been intensely explored, a new field is emerging, termed « RNA epigenetics », the study of RNA modifications. We will discuss about the exciting potential of these modifications to fine-tune mRNA functions, which is changing our perception of epigenetic regulation.

F. Fuks: None.

\section{E03}

Bridging genomic discoveries and personalized medicine

\section{E03.2}

Personalised Medicine and clinical practice: what it means for patients and healthcare delivery

\section{W. G. Newman}

Manchester Centre for Genomic Medicine, University of Manchester, Manchester, United Kingdom

Genomic medicine is a rapidly evolving area. In this talk I will examine some of the drivers for its clinical adoption, explore some of the barriers to this and consider how these may potentially be overcome. I will use examples from the pharmacogenetics of adverse drug reactions and the use of genomic tests to inform treatment choices in cancer to illustrate principles that will ensure that lessons are learnt from past experiences and that these are used to aid design of future studies to facilitate rapid adoption. I will consider the evidence base that is required that will provide confidence to health care commissioners to ensure that genomic medicine is used for patient benefit rather than sitting in a research environment.

W.G. Newman: None.

\section{E04}

\section{Gene Expression Resources}

\section{E04.2}

Tissue-specific enhancer and promoter evolution in mammals

\section{P. Flicek}

European Molecular Biology Laboratory, European Bioinformatics Institute, Hinxton, United Kingdom

Mammals are characterised by a wide range of diverse morphology, behaviour and other characteristics arising from a largely consistent set of genes. A significant fraction 
of these differences are assumed to derive from alterations in transcriptional regulation caused, for example, by species-specific enhancer and promoter usage. Indeed, previous work from our laboratory and others have demonstrated that, within a single tissue, species-specific regulatory elements are extremely common and that enhancers evolve more rapidly than promoters.

To understand the evolution of tissue-specific gene regulation, we have generated genome wide maps of enhancers and promoters as well as matched gene expression data in four tissues-liver, muscle, brain and testes-for 10 diverse mammalian species. For all species, the three somatic tissues show a consistent regulatory profile with the majority of promoters active across multiple tissues, while enhancer activity is mostly tissue specific. Testes are consistently different and display a regulatory profile associated with increased transcriptional activity. We are able to classify enhancers into different types, as evidenced by distinct histone enrichment profiles, and show that they evolve at different rates. By comparing across tissues and across species, we can also characterise elements that are reused in different contexts and suggest a role for transposable elements in tissue-specific and tissue-shared regulatory elements. Together these results provide insight into the evolutionary rewiring of transcriptional regulation and help to understand genome function

P. Flicek: F. Consultant/Advisory Board; Modest; Fabric Genomics, Inc., Eagle Genomics, Ltd..

\section{E05}

\section{The longer the better? Third generation sequencing} technologies

\section{E05.2}

\section{Delineating the structure of chromosome rearrangements using multiple WGS technologies}

\section{A. Lindstrand}

\section{Karolinska Institutet, Stockholm, Sweden}

In recent years, technological advances have improved the turnaround time and cost-effectiveness of human genetic investigations in both diagnostics and research. However, most focus has been on single nucleotide variation (SNV). In contrast, the calling and interpretation of structural variants (SV) is still challenging and causal mutations often go unnoticed in genome studies. Further, the methods applied currently in genetic diagnostics have limitations in detection and resolution and in consequence causal variants may be missed or misinterpreted, resulting in sub-optimal clinical management. Both balanced chromosomal rearrangements and copy number variants (CNVs) as detected by karyotyping or chromosomal microarray might benefit from further investigation by whole genome sequencing (WGS) to accurately resolve the structural rearrangement. We and others have shown that unexpected complexities are common findings in the breakpoints of karyotypically balanced chromosomal rearrangements. Such findings are of clinical importance, as they may be the cause of mendelian phenotypes in the rearrangement carrier. Short read WGS allows for high resolution characterization of SVs, but problems remain for mapping breakpoints located in repetitive regions of the genome, which are known to be prone to rearrangements. In our study, we use multiple complementary WGS experiments to phase and solve the structures of chromosomal rearrangements. In many cases, by delineating the derivative chromosomes we provide a molecular genetic explanation for the clinical symptoms observed in the carrier. Furthermore, we compare the performance, sensitivity and resolution of different WGS techniques in a clinical diagnostic laboratory set.

\section{A. Lindstrand: None.}

\section{E06}

Pharmacogenomic testing for personalized medicine

\section{E06.1}

\section{Integrating pharmacogenomics into personalized drug} treatment

\section{Ingelman-Sundberg ${ }^{1,2}$, M. Ingelman-Sundberg ${ }^{2}$ \\ ${ }^{1}$ Stockholm, Sweden, ${ }^{2}$ Karolinska Institutet, Stockholm, Sweden}

Pharmacogenomic biomarkers for prediction of drug metabolism, toxicity and response are currently approved by FDA and EMA and are implemented into clinical work. These mainly identify common genetic variations in genes encoding enzymes, transporters and targets. However, recent results from analyses of WGS and WES sequencing efforts of large populations reveal that about $40 \%$ of all loss of function (LOF) and missense mutations of importance for prediction of drug response are rare. Pharmacokinetic analyses in twins also reveal that $50 \%$ of the interindividual inherited variation in metoprolol and toresimide pharmacokinetics are caused by mutations not routinely analysed in the pharmacogenetic platforms currently used. This forms a problem for specific individualized drug therapy when the patient specific mutations have to be taken into consideration. The talk will consider the genes and drugs of importance where NGS based sequencing data would 
substantially add information beyond the current platforms to facilitate individualized drug therapy. This includes the description of algorithms able to predict the functionality of missense mutations in the pharmacogenes and protocols for streamline analyses in personalized medicine where the whole genome sequence is taken into consideration.

\section{Ingelman-Sundberg: None.}

\section{E06.2}

Pharmacogenomics based personalized drug treatment across world populations

\section{A. Gaedigk}

\section{Children's Mercy Kansas City, Kansas City, MO, United} States

Cytochrome P450 enzymes including CYP2C9, CYP2C19 and CYP2D6 are among the most extensively studied drug metabolizing enzymes owing to their contribution to the metabolism and/or bioactivation of numerous clinically used drugs. Each exhibits a wide range of activity among individuals within a population and among world populations. Extreme phenotypes can explain adverse drug events or treatment failure. In addition, HLA loci with distinct ethnic and geographical distributions, can cause severe, if not life-threatening adverse drug reactions.

Rich resources for the implementation of pharmacogenetics include the Clinical Pharmacogenetics Implementation Consortium which publishes clinical practice guidelines, the Pharmacogene Variation Consortium, which serves as a centralized repository for allelic variation and the Pharmacogenomics Knowledge Base, which curates knowledge about the impact of genetic variation on drug response for clinicians and researchers. Brief overviews will be provided.

Implementation of pharmacogenetics into clinical practice still faces a number of challenges including testing and test interpretation. As exemplified on CYP2D6, genotype analysis is not trivial due to SNPs, indels, copy number variation and rearrangements with the CYP2D7 pseudogene. Other challenges are the translation of genotype into phenotype and the assignment of function to allelic variants. Furthermore, a distant SNP has been shown to impact CYP2D6 activity in vitro, but there is little known regarding its impact on activity in vivo. Ongoing studies addressing the question whether this SNP needs to be taken into account for phenotype prediction from genotype data will be presented.

Pharmacogenetics is increasingly utilized to individualize drug therapy. However, a pharmacogenetic test may not provide an answer for each patient; thus, much remains to be learned to realize the full potential of pharmacogenetics.

A. Gaedigk: B. Research Grant (principal investigator, collaborator or consultant and pending grants as well as grants already received); Significant; R24 GM123930 and U54 HD090258-01.

\section{E07}

Single-cell transcriptomics in the brain

\section{E07.2}

Single cell RNA sequencing in psychiatric disorders

\section{J. Hjerling Leffler}

\section{Karolinska Institutet, Stockholm, Sweden}

Genome-wide association studies (GWAS) have discovered hundreds of loci associated with complex brain disorders, and provide the best current insights into the etiology of these idiopathic traits. However, it remains unclear in which cell types these variants may be active, which is essential for understanding disease etiology and for disease modelling. Recently we integrated GWAS results with single-cell transcriptomic data from the brain and the entire nervous system to systematically identify cell types underlying psychiatric disorders as well as neurological conditions and other brain complex traits. We show that psychiatric disorders are predominantly associated with excitatory neurons from the cortex/hippocampus, medium spiny neurons from the striatum, diverse sets of midbrain neurons, and inhibitory neurons from the cortex/hippocampus. Cognitive traits were generally associated with similar cell types but their associations were driven by different genes. Neurological disorders were associated with different cell types, consistent with other lines of evidence. Notably, we found that Parkinson's disease is not only genetically associated with dopaminergic neurons but also with serotonergic neurons and cells from the oligodendrocyte lineage. Using post-mortem brain transcriptomic data, we confirmed alterations in these cells, even at the earliest stages of disease progression. Altogether, our study provides a solid framework for understanding the cellular basis of complex brain disorders.

J. Hjerling Leffler: B. Research Grant (principal investigator, collaborator or consultant and pending grants as well as grants already received); Modest; Roche. 


\section{E08}

Chromosome $Y$ loss and the ageing genome

\section{E08.1}

Mosaic loss of chromosome Y (LOY) in leukocytes: from discovery to impact

\section{A. Forsberg}

Uppsala University, Uppsala, Sweden

A growing number of epidemiological investigations show that LOY is linked with morbidity and mortality in men. LOY in blood cells is the most common acquired human mutation and is associated with increased risk for all-cause mortality and disorders such as various forms of cancer, Alzheimer's disease, cardiovascular disease, autoimmune conditions, age-related macular degeneration and type 2 diabetes. As a male specific genetic risk factor, LOY could help explain why men in the entire world live shorter lives than women.

It is not established whether the associations between LOY in leukocytes and risks for disease in other organs represent causal relationships, and if so, what the underlying mechanisms could be. For example, LOY is associated with factors such as age, smoking as well as genetic background, and part of the LOY-associated increased risk for various outcomes might be explained by these confounders. However, we have also proposed a hypothesis involving disturbed functions of blood leukocytes without a Y chromosome and reduced protection against disease in men with LOY. Hence, LOY in immune cells might be responsible for impairment of normal immune system functions such as inflammation and immune-surveillance, responsible for elimination of abnormal cells and structures throughout the soma.

To investigate if LOY changes the normal properties of immune cells we have studied LOY at the levels of DNA-, RNA- and plasma proteome; in bulk, sorted- and singlecells in vivo and in vitro. For example, we have (i) mapped the level of LOY in six major immune cell types in patients diagnosed with Alzheimer's disease and prostate cancer, (ii) studied changes in gene expression in cells with LOY and identified numerous genes showing LOY associated transcriptional effect (LATE) and (iv) found that LOY leaves a footprint in the plasma proteome. These findings support the hypothesis that functions of immune cells is compromized as an effect from LOY, and thus, provide clues toward mechanistic explanations for the associations between LOY in blood cells and increased risk for disease in other organs.

L.A. Forsberg: E. Ownership Interest (stock, stock options, patent or other intellectual property); Modest; CRAY Innovation AB.

\section{E08.2}

Mosaic chromosome $Y$ loss, ageing and cancer risk

\section{J. Machiela}

National Cancer Institute, Rockville, MD, United States

Genetic mosaicism is the acquisition of a somatic mutation or mutations in a clonal subset of cells that differs from the inherited germline genome. Detectable genetic mosaicism is an attractive potential early biomarker for cancer risk because of its established relationship with aging, introduction of potentially deleterious mutations, and clonal selection and expansion of mutated cells. Mosaic loss of the $\mathrm{Y}$ chromosome (mLOY) is the most commonly detected mosaic copy number alteration in men. $\mathrm{mLOY}$ is associated with aging, cigarette smoking and several genetic susceptibility loci. mLOY has also been associated with numerous outcomes including cancer risk and cancer mortality. Shared risk factors for mLOY and associated outcomes makes performing epidemiologic investigations of $\mathrm{mLOY}$ and potential outcomes difficult. While several studies have investigated associations between $\mathrm{mLOY}$ and cancer risk, the relationship remains an active area of research.

M.J. Machiela: None.

\section{E09}

Variant interpretation and high-throughput functional assays

\section{E09.1}

\section{Unraveling the functional impact of thousands of p53} mutations

\section{E. Segal, ${ }^{1,2}$}

${ }^{1}$ Rehovot, Israel, ${ }^{2}$ Weizmann Institute of Science, Rehovot, Israel

The TP53 gene is frequently mutated in human cancer, yet research has focused predominantly on six major "hotspot" codons, which account for only $\sim 30 \%$ of cancer-associated p53 mutations. Here, I will present our work on functional profiling of a library of over 10,000 variants that we generated for the tumor suppressor p53. Remarkably, the mutational effects observed in large-scale in vitro assays with this library correspond to p53 mutation recurrence in patients, and provide novel insights on adverse and benign variants, protein structure, and evolutionary conservation. Apart from gaining comprehensive insights into the effects of the p53 "mutome", our results may lead to better understanding of a patient's response to treatment based on 
their p53 sequence, potentially contributing to the development of novel patient-specific therapeutics.

\section{E09.2}

Understanding the functional effects of coding variation, at scale

\section{M. Starita}

\section{University of Washington, Seattle, WA, United States}

In recent years multiplexed functional assays have fundamentally changed the way we can empirically measure the effects of genetic variation. We can now measure the functional effect of thousands of single nucleotide variants in protein coding genes and other sequence elements such as promoters, enhancers and splice signals in a single experiment. The ability to scale functional assays means that functional scores are more likely to be available as evidence to help guide interpretation of variants of uncertain significance that result from clinical genetic testing. These massive functional datasets already exist for $B R C A 1, T P 53$, PTEN, SUMO, CALM1/2/3, PPARG, TPMT and TPK1. Many more will be generated in the near future and it is likely that one may be generated for your gene of interest. In this educational session, I will first outline the general experimental steps in a multiplexed functional assay. I will then present data sets for BRCAl generated by saturation genome editing (Findlay et al. Nature, 2018) and PTEN generated by an assay for protein abundance (Matreyek et al. Nature Genetics, 2018). Finally, I will discuss the benefits and limitations of using data generated by multiplexed functional assays as evidence for or against pathogenicity for a variant.

L.M. Starita: None.

\section{E10}

Meiosis: factory of genetic variation

\section{E10.1}

Genetic diversity and its unexpected impacts on recombination, genome evolution, speciation and sterility in mammals

\author{
R. Li', E. Bitoun ${ }^{1}$, N. Altemose ${ }^{2}$, R. W. Davies ${ }^{3}$, \\ B. Davies ${ }^{1}$, S. Myers ${ }^{1}$ \\ ${ }^{1}$ University of Oxford, Oxford, United Kingdom, ${ }^{2}$ Univer- \\ sity of California, Berkeley, CA, United States, ${ }^{3}$ The \\ Hospital for Sick Children, Toronto, ON, Canada
}

During meiotic recombination in most mammals, hundreds of programmed DNA Double-Strand Breaks (DSBs) occur across all chromosomes in each cell at sites bound by the protein PRDM9, and resolve into recombination events, which generate new genetic diversity. Conversely mutations in PRDM9 itself, and at binding sites of PRDM9, drive profound differences in the recombination landscapes both among individuals, and between all species possessing this gene so far examined, so diversity impacts recombination. PRDM9 is also the only known mammalian speciation gene, causing meiotic failure and male-limited sterility in certain mouse hybrids. Our work has revealed that this is driven by PRDM9 mainly binding "non-shared" sites that differ between the two homologous genomic copies present in hybrids, due to evolutionary mutations. This leads to unrepaired DSBs, failure to pair chromosomes, and sterility, indicating strong impacts of local sequence divergence on recombinational repair, via still unknown mechanisms. Fertility is fully restored by engineering mice with different, shared, PRDM9 binding sites. We will discuss new work that characterizes the impact of local sequence divergence on mammalian recombination, using thousands of directly detected crossover and non-crossover recombination events from a mouse hybrid cross, resolved with unprecedented power and resolution. Recombination events, but not DSBs, are strongly depleted at the "non-shared" sites. Therefore, mutations within PRDM9 binding sites disrupt and/or inhibit the homologous repair of DSBs. Previous work has found that in humans, GC-biased gene conversion occurs in non-crossovers, driving genome evolution. Unexpectedly, we found that in both mice and humans, this bias occurs exclusively in non-crossovers containing just a single mismatch - with no GC-bias whatsoever in non-crossovers with multiple mismatches. These results demonstrate that alongside altering where recombination initiates, local genetic diversity also profoundly alters meiotic repair pathway decisions via at least two distinct mechanisms, impacting genome evolution and Prdm9related hybrid infertility.

R. Li: None. E. Bitoun: None. N. Altemose: None. R. W. Davies: None. B. Davies: None. S. Myers: None.

E10.2

Meiotic recombination, gene conversion and mutation

\section{Tiemann-Boege ${ }^{1}$, A. Heissl ${ }^{1}$, A. J. Betancourt ${ }^{2}$,} B. Arbeithuber ${ }^{3}$

${ }^{1}$ Johannes Kepler University, Linz, Austria, ${ }^{2}$ University of Liverpool, Liverpool, United Kingdom, ${ }^{3}$ Penn State University, State College, PA, United States 
During meiosis the maternal and paternal genetic material is exchanged in a highly regulated process known as meiotic recombination. Recombination is initiated by the formation of double strand-breaks (DSBs), and in most organisms it is clustered in narrow regions, called recombination hotspots. The repair of DSBs reshapes the nucleotide landscape at recombination hotspots and a high number of polymorphisms accumulate at hotspots over time. In humans and mice, these polymorphisms lead to changes in affinity of PRDM9, a meiosis-specific, trans-acting protein that recognizes DNA target motifs and activates recombination hotspots. We still do not fully understand the exact molecular mechanism driving the sequence erosion at hotspots, but it has important consequences in the outcome of recombination. We have characterized the processes re-shaping the nucleotide sequences at recombination hotspots by screening a large number of single recombination molecules. We particularly focused on de novo mutations and gene conversion events, as well as the effect of poly-A repeats of different lengths. The repair of DSBs can lead to a biased nucleotide composition at recombination hotspots by gene conversion, which is the non-reciprocal exchange of DNA stretches of one chromosome to the other and is often associated with a higher probability of transmitting one allele over the other. At recombination hotspots, the transmission of GC over AT variants are favoured resulting over time to a higher GC content at hotspots. Similarly, we observed that longer poly-A repeats are transmitted more often than shorter repeats resulting in an insertion biased gene conversion, albeit this depends on the nature of the repeat heterology. We also found that hotspots are enriched with de novo mutations, especially at $\mathrm{CpGs}$ sites that are prone to a high spontaneous deamination rate in the context of single stranded DNA formed during DSB repair. Meiotic recombination leads to the sequence erosion at hotspots that in turn affects the binding of the recombination machinery and eliminates hotspots over time. In other words, hotspots have a tendency to self-destruct through the systematic over-transmission of alleles and accumulation of mutations, which poses the question if trans-acting factors evolve as a response to these effects. Project funded by the Austrian Science Fonds (FWF) P23811000 and P308667000.

I. Tiemann-Boege: None. A. Heissl: None. A.J. Betancourt: None. B. Arbeithuber: None.

\section{E11}

Genome First Testing in Pediatrics

\section{E11.1}

The landscape of genomic alteration across childhood cancers
N. Jäger ${ }^{1,2}$, B. C. Worst ${ }^{1,3}$, D. T. W. Jones ${ }^{1,3,4}$, S. M. Pfister ${ }^{1,2,5}$

${ }^{I}$ Hopp Children's Cancer Center Heidelberg (KiTZ), Heidelberg, Germany, ${ }^{2}$ Department of Pediatric Neurooncology, German Cancer Research Center (DKFZ), Heidelberg, Germany, ${ }^{3}$ Pediatric Glioma Research (B360), German Cancer Research Center (DKFZ), Heidelberg, Germany, ${ }^{4}$ German Cancer Consortium (DKTK), Heidelberg, Germany, ${ }^{5}$ Department of Pediatric Hematology and Oncology, Heidelberg University Hospital, Heidelberg, Germany

Cure rates for childhood cancers have increased to about $80 \%$ in recent decades, but cancer is still the leading cause of death by disease in the developed world among children over one year of age. Furthermore, children who survive cancer often suffer from the long-term side-effects of therapy. A crucial step in developing more specific and less damaging therapies is to map the complete (epi-)genetic repertoire of pediatric malignancies, which is highly relevant for the design of future clinical trials and for improving translational impact.

Through the advent of genome-wide molecular profiling, specifically next-generation sequencing, it became evident how heterogeneous and distinct childhood cancers are in comparison to the often environmentally-associated adult cancers. The recent large-scale pan-cancer analyses of pediatric malignancies have demonstrated differences in tumor mutational burden and mutational signatures, as well as unique driving events. The role of pathogenic germline variants predisposing to cancer is particularly important in this patient population, likely being involved in at least $10 \%$ of cases of childhood tumors.

Central nervous system (CNS) tumors are the most common type of solid tumor in children and the leading cause of cancer-related death in this age group. Many of the discoveries which define the landscape of genomic alteration across childhood cancers were made in this highly relevant entity, since pediatric CNS tumors have been extensively sequenced in large consortia and independent groups alike. Major genetic discoveries range from highly recurrent histone $\mathrm{H} 3$ mutations, and hijacking of distal enhancer elements, to new oncogenic gene fusions (of the NTRK family of kinases, among others). Further, chromatin modifiers were found to be recurrently altered in childhood brain tumors.

This presentation focuses on key findings that have been transforming the landscape of pediatric cancer research in the past decade and how these results are opening new avenues towards therapeutic approaches.

N. Jäger: None. B.C. Worst: None. D.T.W. Jones: None. S.M. Pfister: None. 


\section{E11.2}

\section{Rapid NGS for children in intensive care units}

\section{F. Raymond}

\section{University of Cambridge, Cambridge, United Kingdom}

With growing evidence that rare single gene disorders present in the neonatal period, there is a need for rapid, systematic, and comprehensive genomic diagnoses in ICUs to assist acute and long-term clinical decisions. We performed trio whole genome sequence analysis (WGS) on a prospective cohort of families recruited in NICU and PICU at a single site in the UK. We developed a research pipeline in collaboration with the National Health Service (NHS) to deliver validated pertinent pathogenic findings within 2-3 weeks of recruitment. The analysis includes single nucleotide variants (SNV), copy number variants (CNV), structural variants (SV), uniparental disomy, mitochondrial genome analysis and specific analysis of trinucleotide repeat diseases including myotonic dystrophy and Spinomuscular atrophy (SMA). $>330$ families had whole genome analysis performed and $20-25 \%$ received a molecular diagnosis for the underlying genetic condition in the child. The phenotypic description of the child was a poor predictor of the gene identified in $90 \%$ of cases, arguing for gene agnostic testing in NICU/PICU. The diagnosis affected clinical management in $>65 \%$ of cases including modification of treatments and care pathways and/ or informing palliative care decisions. A 2-3 week turnaround was sufficient to impact most clinical decision making. The use of WGS in intensively ill children is acceptable and trio analysis facilitates diagnoses. A "gene agnostic" approach was effective in identifying an underlying genetic condition, with phenotypes and symptomatology being primarily used for data interpretation rather than gene selection. Whole Genome Sequence analysis has the potential to be a first line diagnostic tool for a subset of intensively ill children.

\section{F. Raymond: None.}

\section{E12}

\section{Oligogenic inheritance}

\section{E12.1}

Systematic analysis of genetic interactions: from yeast to human

\section{J. Van Leeuwen}

University of Lausanne, Lausanne, Switzerland
The functional consequence of a mutation is frequently dependent on the genetic background in which it occurs, which complicates the identification of complete sets of causal variants associated with phenotypes, including many diseases. These differences can be the result of modifying mutations in other genes, that can either increase the severity of a disease phenotype, or that can have a protective effect and compensate for the deleterious effects of the disease mutation. Although identification of modifier loci can highlight potential avenues for therapeutic intervention, it requires data from large cohorts of patients carrying the same disease allele. Given the challenges associated with identifying modifier mutations in humans, genetic interactions have mainly been studied using model organisms. The budding yeast Saccharomyces cerevisiae has often been the model organism of choice for systematic studies of genetic interactions due to its highly annotated genome and tractable genetics. Mapping genetic interactions in model organisms provides a powerful approach for dissecting gene function and pathway connectivity, and for defining conserved properties of genetic interactions that can elucidate genotype-to-phenotype relationships. In addition, with the development of CRISPR-Cas9 technology, we can now screen for genetic interactions directly in cultured human cells. I will discuss how we can use systematic genetic interaction screens in yeast and cultured human cells to define conserved properties of genetic interactions, and how we are starting to use these to identify genetic interactions between variants in human genome sequences.

\section{J. Van Leeuwen: None.}

\section{E13}

\section{Genetic innovations in reproductive medicine}

\section{E13.1}

Novel discoveries of genes implicated in male and female infertility

\section{Arnoult ${ }^{1,2}$, P. Ray $^{3}$}

${ }^{1}$ University of Grenoble Alpes, Grenoble, France, ${ }^{2}$ Institut pour l'Avancée des Biosciences (IAB), INSERM 1209, CNRS UMR 5309, Grenoble, France, ${ }^{3} \mathrm{CHU}$ Grenoble Alpes, Grenoble, France

The high-throughput sequencing has become a very efficient and low cost approach to identify mutations leading to human diseases. The number of genes linked to human pathologies has grown exponentially these last years and the domain of human infertility did not escape this global trend. Our laboratory, in collaboration with several 
fertility clinics located in Europe, Africa and Asia, has gathered a large cohort of infertile males and females presenting with different marked phenotypes, such as acrosome-less sperm showing a round shape (globozoospermia), sperm with flagellum defects (teratozoospermia with Multiple Morphological Anomalies of the Flagella, known as MMAF), absence of sperm in the ejaculate (azoospermia), or oocytes blocked during meiotic maturation. Exomic sequencing of these patients enable us to identify 13 genes related to male infertility and 1 gene to female infertility, so far. In the MMAF cohort, a genetic cause has been identified in half of the patients but have shown a high genetic heterogeneity, highlighting the abundance of genes involving in flagellum biogenesis. The function and the localization of the corresponding proteins were studied using the most suitable deficient animal models, including mouse and Trypanosoma brucei, a protozoan flagellate parasite for genes related to MMAF. Through several examples, this presentation aims at showing how the discovery of genes involved in human infertility allow to improve both diagnosis and prognosis in human infertility but also enable to identify new molecular pathways necessary for mammalian gametogenesis.

C. Arnoult: None. P. Ray: None.

\section{E13.2}

Population genetic carrier screening programs for reproductive purposes

\section{J. Zlotogora}

Hadassah medical center, Hebrew University, Jerusalem, Israel

The aim of population genetic carrier screening programs for reproductive purposes is to allow couples at risk to choose between all the available options before a pregnancy. Population genetic carrier screening programs may be voluntary or required either by law or by the religious authorities before marriage being targeted to founder populations or offered to the whole population. The Israeli population genetic carrier screening program for reproductive purposes is voluntary, available to all the population and free of charge. The program is used by most of the population at reproductive age and the tests are done either before marriage, before conception or during pregnancy. The program includes screening for all the severe diseases that are known to be relatively frequent in the population and in which a test allows the detection of more than $90 \%$ of the carriers. Carrier screening for cystic fibrosis, SMA and FraX is recommended to all the population while for other diseases the recommendation is limited to the communities at risk: among Jews according to the community of origin and among Arabs and Druze according to the religion and locality of origin. The extraordinary scientific changes that occurred in the last decade revolutionized the offers of private carrier screening programs and pilots for population programs are ongoing in several countries. The best option may be a universal screening panel with a preference for couple screening giving results only when the couple is at risk for a severe disease in a future child.

\section{J. Zlotogora: None.}

\section{Concurrent Sessions}

\section{C01}

Novel diagnostic approaches

\section{C01.1}

A national approach to rapid genomic diagnosis in acute paediatrics

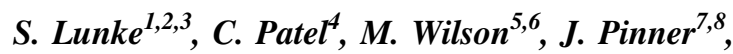
S. A. Sandaradura ${ }^{5,6}$, D. Mowat ${ }^{7,8}$, E. Kirk ${ }^{7,8,9}$, M. F. Hunter ${ }^{10,11}$, E. I. Krzesinski ${ }^{10,11}$, C. Barnett ${ }^{12}$, L. S. Akesson ${ }^{1,3,10}$, C. M. Richmond ${ }^{1}$, S. Kumble ${ }^{1}$, N. B. Tan ${ }^{1}$, A. Fennell ${ }^{10,11}$, J. Rogers ${ }^{4}$, M. Higgins ${ }^{4}$, A. Vasudevan ${ }^{13}$, K. B. Howell ${ }^{3,14,15}$, S. M. White ${ }^{1,3}$, M. G. de Silva ${ }^{1,2}$, G. R. Brett ${ }^{1,3,16}$, L. Gallacher ${ }^{1,3}$, S. Ayres ${ }^{1,2,16}, \mathrm{~K}$. Boggs ${ }^{5,7,2}$, A. Bray ${ }^{2,5,7}$, A. Baxendale ${ }^{12}$, S. Borrie ${ }^{12}$, S. King-Smith ${ }^{2,12}$, M. C. Quinn ${ }^{2,4}$, L. Fowles ${ }^{4}$, L. Hunt ${ }^{4}$, S. Eggers', J. Riseley ${ }^{1}$, B. Chong ${ }^{1}$, D. Phelan ${ }^{1}$, S. Sadedin', M. Martyn ${ }^{14,16}$, I. Goranitis ${ }^{2,3}$, S. Best ${ }^{2,17}$, M. F. Buckley', T. Roscioli ${ }^{9,7,8}$, J. Christodoulou ${ }^{1,2,3}$, Australian Genomics Health Alliance Acute Care Flagship, Z. Stark ${ }^{1,2,3}$

${ }^{1}$ Victorian Clinical Genetics Service, Melbourne, Australia, ${ }^{2}$ Australian Genomics Health Alliance, Melbourne, Australia, ${ }^{3}$ University of Melbourne, Melbourne, Australia, ${ }^{4}$ Genetic Health Queensland, Royal Brisbane and Women's Hospital, Brisbane, Australia, ${ }^{5}$ Sydney Children's Hospital Network-Westmead, Sydney, Australia, ${ }^{6}$ University of Sydney, Sydney, Australia, ${ }^{7}$ Sydney Children's Hospital Network-Randwick, Sydney, Australia, ${ }^{8}$ University of New South Wales, Sydney, Australia, ${ }^{9}$ NSW Health Pathology Randwick Genomics Laboratory, Sydney, Australia, ${ }^{10}$ Monash Genetics, Monash Children's Hospital, Melbourne, Australia, ${ }^{11}$ Department of Paediatrics, Monash University, Melbourne, Australia, ${ }^{12}$ Women's and Children's Hospital, Adelaide, Australia, ${ }^{13}$ Royal Women's Hospital, Melbourne, Australia, ${ }^{14}$ Murdoch Children's Research Institute, 
Melbourne, Australia, ${ }^{15}$ Royal Children's Hospital, Melbourne, Australia, ${ }^{16}$ Melbourne Genomics Health Alliance, Melbourne, Australia, ${ }^{17}$ Australian Institute of Health Innovation, Macquarie University, Sydney, Australia

Introduction: Implementation of rapid genomic testing in neonatal and paediatric intensive care units (NICUs/PICUs) is gathering momentum, and requires the development of systems capable of consistent delivery across multi-site networks.

Methods: We developed a rapid genomic diagnosis program involving 10 Australian hospitals and two laboratories with the aim of providing test results in $<5$ days for acutely unwell paediatric patients with suspected monogenic disorders. Rapid exome sequencing (rES) was performed as trios when possible, and analysis utilised multidisciplinary expertise. Experience was shared between clinical sites, laboratories, and professional groups to enable collective learning.

Results: The program considered 123 patients for rES over 10 months, and approved 114 (93\%). Five families declined testing (4.4\%), and nine (7.9\%) were withdrawn due to change in clinical circumstances. Of 100 patients tested, 51 received a diagnosis. Eleven of the diagnoses $(21 \%)$ were made using approaches augmenting standard ES analysis: mitochondrial genome sequencing, ES-based copy number analysis, matchmaking of emerging genes, reverse phenotyping and RNA analysis. Median time from hospital admission to consent was 6 days (range 0-64 days); median time from sample receipt to clinical ES report was 3 days (range 2-7 days). The total cost of testing was AU\$1,123,000/€701,638 (AU\$11,230/€7,016 per case). Changes in management following a result occurred in $77 \%$ of diagnosed patients and $10 \%$ of undiagnosed patients.

Conclusion: We demonstrate the feasibility of a national, highly integrated clinical-laboratory approach to rapid genomic diagnosis, which delivers results within a timeframe relevant to acute paediatrics, while optimising clinical utility and resource allocation.

S. Lunke: None. C. Patel: None. M. Wilson: None. J. Pinner: None. S.A. Sandaradura: None. D. Mowat: None. E. Kirk: None. M.F. Hunter: None. E.I. Krzesinski: None. C. Barnett: None. L.S. Akesson: None. C. M. Richmond: None. S. Kumble: None. N.B. Tan: None. A. Fennell: None. J. Rogers: None. M. Higgins: None. A. Vasudevan: None. K.B. Howell: None. S.M. White: None. M.G. de Silva: None. G.R. Brett: None. L. Gallacher: None. S. Ayres: None. K. Boggs: None. A. Bray: None. A. Baxendale: None. S. Borrie: None. S. KingSmith: None. M.C. Quinn: None. L. Fowles: None. L. Hunt: None. S. Eggers: None. J. Riseley: None. B. Chong: None. D. Phelan: None. S. Sadedin: None. M.
Martyn: None. I. Goranitis: None. S. Best: None. M.F. Buckley: None. T. Roscioli: None. J. Christodoulou: None. Z. Stark: None.

\section{C01.2}

Effectiveness of integrated interpretation of exome and corresponding transcriptome data in detecting splicing variants of recessive disorders

\section{Yamada', Y. Shiraishi ${ }^{2}$,H. Suzuki ${ }^{1}$, K. Kosaki ${ }^{1}$ \\ ${ }^{1}$ Keio University School of Medicine, Shinjuku, Japan, ${ }^{2}$ National Cancer Center Research Institute, Tokyo, Japan}

Background: Incorporation of RNA-seq data into exome analyses could help detection of abnormal splicing efficiently. Recently, Shiraishi developed a novel algorithm, "SAVNet: Splicing-Associated Variants NET", allowing the integrated analysis of RNA-seq and exome data from cancer tissues.

Methods: We applied the SAVNet algorithm to decipher abnormal splicing events and examined the correlation between these events and rare germline variants (MAF $<0.03$ ) among 179 phenotypically normal subjects whose RNA-seq data (lymphoblastoid cell lines) and corresponding exome data were publicly available. Only the 1913 causative genes of known autosomal recessive disorders were evaluated under the premise that the subjects could be carriers.

Results: Among 1913 genes, 1272 had expression levels that were sufficient to be analyzed by SAVNet. Forty aberrant splicing events associated rare germline variants were detected in 31 of the 179 subjects; creation of alternative $5^{\prime}$ splice site (13/40 events), creation of alternative $3^{\prime}$ SS (14) and exon skipping (13). The predicted effects on protein were as follows: mutation out of frame (30), and inframe (10). Intriguingly, seven variants annotated as "missense" (5) or "silent" (2) per exome analyses had triggered abnormal splicing that disrupted the reading frame. Conventional exome analysis the 1272 genes detected a total of 144 putatively pathogenic variants including nonsense, frameshift, and canonical splice site (gt/ag) mutations.

Discussion: Coupling interpretation of personal genomes with their corresponding transcriptomes successfully uncovered pathogenic splicing variants. Using this method, the detection rate of provisionally truncating pathogenic variants increased by twenty percent (28 / 144) in comparison with conventional exome analysis.

M. Yamada: None. Y. Shiraishi: None. H. Suzuki: None. K. Kosaki: None.

\section{C01.3}

Chromosome conformation capture (HiC) combined 
with whole genome sequencing for the detection and functional interpretation of complex genomic rearrangements in developmental disease

\section{U. S. Melo ${ }^{I}$, R. Schöpflin ${ }^{I}$, R. A. Hidalgo ${ }^{I}$,} M. A. Mensah ${ }^{2}$, B. Fischer-Zirnsak ${ }^{2}$, V. Suckow ${ }^{1}$, S. Türkmen ${ }^{2}$, F. Quintero ${ }^{3}$, S. B. Sousa ${ }^{4}$, P. Louro ${ }^{5}$, M. Spielmann ${ }^{1}$, V. Kalscheuer ${ }^{1}$, I. Datkhaeva ${ }^{6}$, S. Mundlos ${ }^{1}$

${ }^{1}$ MPI, Berlin, Germany, ${ }^{2}$ Charite, Berlin, Germany, ${ }^{3}$ UCLA, Los Angeles, CA, United States, ${ }^{4}$ Coimbra's Paediatric Hospital, Medical Genetics Department, Coimbra, Portugal, ${ }^{5}$ Coimbra's Paediatric Hospital, Medical Genetics Department, Coimbra, Portugal, ${ }^{6}$ Department of Obstetrics and Gynecology David Geffen School of Medicine, University of California at Los Angeles, Los Angeles, CA, United States

High-throughput based technologies, such as array CGH and whole-genome sequencing have greatly advanced the field of human genetics by identifying and associating structural variants (SVs) with genetic disorders. However, the identification interpretation of SVs often remains difficult, in particular in complex chromosomal rearrangements and when SVs do not disrupt protein-coding sequence. Here, we address these issues by using chromosome conformation capture ( $\mathrm{HiC})$ in patient cell lines in combination with whole-genome sequencing. Rearrangements of the patient's genome with respect to a reference genome emerge as distinct patterns in the HiC contact map. Additionally, HiC informs about the wiring of so-called Topologically Associating Domains (TADs), which have been shown to largely define the genomic range in which genes interact with enhancer elements. HiC was generated from fibroblasts or lymphoblasts from 3 individuals with complex rearrangements at the SOX9 locus and another 5 with interchromosomal translocations. HiC was instructive in revealing the complexity of rearrangements that involved previously unrecognized deletions and insertions. The formation of novel TADs promoting ectopic enhancerpromoter interaction was observed. HiC identified complex nested chromosomal translocations and revealed new enhancer-promoter interactions upon chromosome fusion. HiC performed in LCLs from one patient with chromothripsis showed a complex pattern of interchromosomal translocations, which were further validated by orthogonal methods (e.g M-FISH). In summary, HiC can help to detect and to resolve SVs. The interpretation of SVs is supported by showing the emergence of new or altered TADs making $\mathrm{HiC}$ to a powerful tool to investigate the pathomechanism underlying developmental disorder.
U.S. Melo: None. R. Schöpflin: None. R.A. Hidalgo: None. M.A. Mensah: None. B. Fischer-Zirnsak: None. V. Suckow: None. S. Türkmen: None. F. Quintero: None. S. B. Sousa: None. P. Louro: None. M. Spielmann: None. V. Kalscheuer: None. I. Datkhaeva: None. S. Mundlos: None.

\section{C01.4}

Expanding Next Generation Phenotyping on clinical notes and hand radiographs

Y. Hanani, A. Superti-Furga ${ }^{2}$, H. Warren ${ }^{3}$, Y. Gurovich, N. Fleischer ${ }^{\text {, S. S. A. Skinner }}{ }^{3}$, K. W. Gripp ${ }^{4}$, G. Nadav', P. M. Krawitz ${ }^{5}$

${ }^{1}$ FDNA Inc, Boston, MA, United States, ${ }^{2}$ Division of Genetic Medicine, University of Lausanne, Centre Hospitalier Universitaire Vaudois, Lausanne, Switzerland, ${ }^{3}$ Greenwood Genetic Center, Greenwood, SC, United States, ${ }^{4}$ Division of Medical Genetics, A. I. du Pont Hospital for Children/Nemours, Wilmington, DE, United States, ${ }^{5}$ Institute for Genomic Statistic and Bioinformatics, University Hospital Bonn, Rheinische-Friedrich-Wilhelms University, Bonn, Germany

Next Generation Phenotyping (NGP), and specifically automated facial image analysis, has shown to be useful in the diagnosis of rare disorders. This can be seen in different stages of the diagnostic workup: first, as shown in a recent publication, the DeepGestalt (FDNA Inc.USA) technology utilizes deep-learning algorithms to help clinicians arrive to differential diagnoses and to guide them into which tests to order. Secondly, in an improved variant prioritization as shown in an approach called PEDIA, tested in a retrospective study of 679 diagnosed individuals resulting in significant diagnostic yield improvement (top10 -accuracy rate of $99 \%$ for disease-causing gene). And lastly, potentially assisting in re-evaluation of undiagnosed cases.

Here, we present two additional NGP capabilities: automated clinical notes analysis and hand radiographs analysis. We demonstrate how the technology improves phenotype extraction thus potentially improving diagnostic yield by enabling accurate and consistent interpretation of massive clinical data. We created a benchmark by manually annotating HPO-terms from 126 clinical notes. Results show precision $=0.78, \quad$ recall $=0.41$ and $\mathrm{F}$-score $=0.51$ in extracting correct terms from notes, surpassing previous methods.

Hand radiograph analysis correctly classified 40 of 41 of the patients of three syndromes (Acrodysostosis, CartilageHair-Hypoplasia, SEMD-JL-Leptodactylic) indicating that fine-grained features like "cone-shaped epiphyses" or high- 
level features such as "short-broad hand" can be extracted from this imaging data.

This analysis shows that a more holistic view of patients using NGP technology, can improve the classification results on patients with rare syndromic disorders whose characteristic gestalt is not limited to the facial phenotype alone.

Y. Hanani: A. Employment (full or part-time); Significant; FDNA Inc. A. Superti-Furga: None. H. Warren: None. Y. Gurovich: A. Employment (full or part-time); Significant; FDNA Inc. N. Fleischer: A. Employment (full or part-time); Significant; FDNA Inc. S.A. Skinner: None. K.W. Gripp: A. Employment (full or part-time); Modest; FDNA Inc. G. Nadav: None. P.M. Krawitz: A. Employment (full or part-time); Modest; FDNA Inc.

\section{C01.5}

Using UK Biobank to assess the pathogenicity, penetrance and expressivity of monogenic disease variants

\section{F. Wright, M. A. Tuke, B. West, S. Jones, K. Patel,}

T. W. Laver, R. N. Beaumont, J. Tyrrell, A. R. Wood,

A. Murray, A. T. Hattersley, T. M. Frayling,

\section{N. Weedon}

\section{Institute of Biomedical and Clinical Science, Exeter, United} Kingdom

Introduction: The true penetrance of many of rare diseasecausing alleles is uncertain and may be over-estimated by clinical ascertainment. Here, we use data from 388,714 UK Biobank participants to assess putatively clinically-relevant rare variants in a population-based setting.

Materials and Methods: Although rare variants are harder to genotype accurately than common variants, we were able to classify 1,244 of 4,585 putatively clinicallyrelevant variants genotyped on the UK Biobank SNP-array as high-quality. We defined 'clinically-relevant' as variants that were either classified as pathogenic/likely pathogenic in ClinVar or are in genes known to cause two specific monogenic diseases: maturity-onset diabetes of the young and severe developmental disorders. We also called and visually-inspected 96 copy number variants overlapping regions known to cause developmental delay, as well as large chromosomal events across the genome. We tested the association of these variants with 401 clinically-relevant traits in UK Biobank.

Results: We identified 27 putatively clinically-relevant rare sequence variants and 12 rare copy number variants associated with traits in UK Biobank, exhibiting reduced penetrance or variable expressivity compared with their associated monogenic disease. Within our two disease subsets, we found 15 rare CNVs intersecting known disease genes, and were able to refine the penetrance estimate for a pathogenic $H N F 4 A$ variant in diabetes (only $\sim 10 \%$ by age $40 y$ rs in UK Biobank versus $\sim 75 \%$ in a large monogenic diabetes cohort) and refute the previous disease-association of RNF135 in developmental disorders.

Conclusion: This study shows that large populationbased studies will help refine the penetrance estimates of rare variants.

C.F. Wright: None. M.A. Tuke: None. B. West: None. S. Jones: None. K. Patel: None. T.W. Laver: None. R.N. Beaumont: None. J. Tyrrell: None. A.R. Wood: None. A. Murray: None. A.T. Hattersley: None. T.M. Frayling: None. M.N. Weedon: None.

C01.6

Man vs Machine: Implementing clinically validated automated variant prioritisation with diagnostic performance that equals human experts

L. Burnett ${ }^{1,2,3}$, A. L. Statham ${ }^{4}$, B. A. Lundie $^{4}$, E. Lee Le, $^{2,4}$ A. Poulet ${ }^{1,5}$

${ }^{1}$ Kinghorn Centre for Clinical Genomics, Garvan Institute of Medical Research, Darlinghurst Sydney, Australia, ${ }^{2}$ Genome.One, Australian Clinical Laboratories, Darlinghurst Sydney, Australia, ${ }^{3}$ St Vincent's Clinical School, UNSW Sydney, Darlinghurst Sidney, Australia, ${ }^{4}$ Genome. One, Garvan Institute of Medical Research, Darlinghurst Sydney, Australia, ${ }^{5}$ Institut des Sciences Pharmaceutiques et Biologiques - Faculté de Pharmacie de Lyon, Lyon, France

Introduction: Analysis and interpretation of genomes (and exomes) are major challenges requiring bioinformatics and professional resources in limited supply. We report development of an automated variant prioritisation framework, whose performance equals state-of-the-art clinical genomic diagnosis using human experts.

Methods: Genome.One operates a clinically accredited (ISO15189) whole genome diagnostic service in Sydney, Australia. A reference set of $\sim 500$ clinical referrals underwent bioinformatics using a precisionFDA Award-winning pipeline (Kinghorn Centre for Clinical Genomics). Tertiary bioinformatics used published tools (Seave), with every case analysed by Doctoral genetics professionals. Additionally, a team of genetics experts performed variant review of selected non-trivial cases.

We evaluated two automated pipelines: Moon (diploid. com), Eclipse (derived from Seave), and a combination of the two. Reference set concordance was required for ACMG Class 4 and 5 variants, and desirable (but not 
required) for Class 3 variants. Discordant results were adjudicated by the variant review expert team.

Results: Both automated pipelines performed nearly as well as human experts, identifying at least $88-98 \%$ of Class $4 / 5$ and $60-75 \%$ of Class 3 variants. Applying both in combination equalled human experts, with $100 \%$ concordance for all Class $4 / 5$ reportable variants and $75-81 \%$ concordance for Class 3 variants. The new approach resulted in order-of-magnitude reduction (median reduced from 225 to 6 variants) in interpretation time and need for manual curation and review.

Conclusion: An automated variant prioritisation protocol has been developed, whose performance equals that of credentialed clinical genetic diagnostic expert professionals. The pipeline has been validated and is accredited for clinical diagnosis.

L. Burnett: A. Employment (full or part-time); Significant; Garvan Institute of Medical Research, Genome. One, Australian Clinical Laboratories. B. Research Grant (principal investigator, collaborator or consultant and pending grants as well as grants already received); Modest; Avant (unrelated to this project). B. Research Grant (principal investigator, collaborator or consultant and pending grants as well as grants already received); Significant; Shire International (unrelated to this project). A.L. Statham: None. B.A. Lundie: A. Employment (full or part-time); Significant; Queensland Health Pathology Service. E. Lee: A. Employment (full or part-time); Significant; Genome. One, Australian Clinical Laboratories. A. Poulet: B. Research Grant (principal investigator, collaborator or consultant and pending grants as well as grants already received); Modest; Student scholarship, University of Lyon.

\section{$\mathrm{C02}$}

3D gene regulation

\section{C02.1}

CRISPR-engineered serial genomic inversions lead to tissue-specific architectural stripes, ectopic gene expression and congenital limb malformations

\section{K. Kraft ${ }^{1,2,3}$, A. Magg ${ }^{1}$, V. Heinrich ${ }^{1}$, \\ C. Riemenschneider ${ }^{1}$, R. Schöpflin ${ }^{1}$, J. Markowski ${ }^{I}$, \\ D. Ibrahim ${ }^{1,2}$, R. Acuna-Hidalgo ${ }^{1,2}$, A. Despang ${ }^{1}$, \\ G. Andrey ${ }^{1,2}$, L. Wittler ${ }^{1}$, B. Timmermann ${ }^{1}$, M. Vingron ${ }^{1}$, S. Mundlos ${ }^{1,2}$}

${ }^{1}$ Max Planck Institute for Molecular Genetics, Berlin, Germany, ${ }^{2}$ Charité Universitätsmedizin Berlin, Berlin, Germany, ${ }^{3}$ Stanford University, Stanford, CA, United States
Balanced chromosomal rearrangements (BCRs) such as inversions and translocations can cause congenital disease or cancer by inappropriately rewiring promoter-enhancer contacts. However, it remains difficult to predict if and when aberrant gene activation takes place in the context of BCRs. We have addressed the effect of BCRs on gene expression in vivo by generating a series of genomic inversions that place an active limb enhancer cluster from the Epha4 regulatory domain at different positions within a neighboring gene-dense region. Expression studies in embryonic limb buds show that the inverted enhancer cluster was able to activate several genes downstream of its new position, resulting in gene misexpression and limb phenotypes. Capture Hi-C from mutant limb buds showed that the activated genes were located within a region with an asymmetric three-dimensional pattern, so called architectural stripes. Deletion of the CTCF binding site at the anchor of the stripe resulted in its disappearance, diminished ectopic gene expression, and a rescue of the skeletal phenotype. Moreover, unlike in the developing limb, inversion-derived stripes do not form in mouse embryonic stem cells (mESC) where the enhancers are inactive, indicating that its formation is likely to be dependent on enhancer activity. Based on Hi-C from limb buds we show that architectural stripes are a frequent feature of the chromatin structure in vivo and often associate with developmentally active enhancers. Thus, BCRs can induce ectopic gene expression and the formation of asymmetric chromatin contact pattern that are dependent on CTCF anchors and enhancer activity.

K. Kraft: None. A. Magg: None. V. Heinrich: None. C. Riemenschneider: None. R. Schöpflin: None. J. Markowski: None. D. Ibrahim: None. R. Acuna-Hidalgo: None. A. Despang: None. G. Andrey: None. L. Wittler: None. B. Timmermann: None. M. Vingron: None. S. Mundlos: None.

C02.2

Functional dissection of TADs reveals non-essential and instructive roles in regulating gene expression

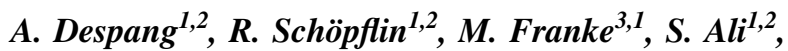 I. Jerkovic, , C. Paliou ${ }^{1}$, W. Chan ${ }^{5}$, B. Timmermann ${ }^{1}$, L. Wittler ${ }^{1}$, M. Vingron ${ }^{1}$, S. Mundlos ${ }^{1,2,5}$, D. M. Ibrahim ${ }^{1,2,5}$

${ }^{I}$ Max Planck Institute for Molecular Genetics, Berlin, Germany, ${ }^{2}$ BIH Center for Regenerative Therapies, Berlin, Germany, ${ }^{3}$ Centro Andaluz de Biología del Desarrollo $(C A B D)$, Sevilla, Spain, ${ }^{4}$ Institute de Genetique Humaine, Montpellier, France, ${ }^{5}$ Charité Berlin - Institut für Medizinische und Humangenetik, Berlin, Germany 
The genome is organized in megabase-sized three-dimensional units, called Topologically Associated Domains (TADs), that are separated by boundaries. TADs bring distant cis-regulatory elements into proximity, a process dependent on the cooperative action of cohesin and the DNA binding factor CTCF. Surprisingly, genome-wide depletion of CTCF has little effect on transcription, yet structural variations affecting TADs have been shown to cause gene misexpression and congenital disease. Here, we investigate the importance of TADs for gene regulation in vivo in mice by systematically editing CTCF-sites, TAD boundary position, and TAD substructure orientation at the Sox9/Kcnj locus.

We find that TADs are formed by a redundant system of CTCF sites and fusion of two neighboring TADS requires the removal of all major sites within the TAD and at the boundary. However, this TAD fusion only results in leakage of regulatory activity from the Sox 9 to the Kcnj TAD, but no major changes in gene expression. In contrast, inversions and/or the re-positioning of boundaries actively re-organize the $3 \mathrm{D}$ chromatin structure and thereby re-direct regulatory activity, resulting in drastic gene misexpression and disease phenotypes.

Our results suggest that TAD structures provide robustness and precision, but are not essential for developmental gene regulation. In structural variations, efficient re-wiring of enhancer promoter interaction and aberrant disease causing gene activation is not induced by a mere loss of insulation, but requires the re-direction of chromatin contacts. Thereby, our results provide a basis to predict the regulatory effects of structural variations.

A. Despang: None. R. Schöpflin: None. M. Franke: None. S. Ali: None. I. Jerkovic: None. C. Paliou: None. W. Chan: None. B. Timmermann: None. L. Wittler: None. M. Vingron: None. S. Mundlos: None. D.M. Ibrahim: None.

\section{C02.3}

Saturation mutagenesis of disease-associated regulatory elements

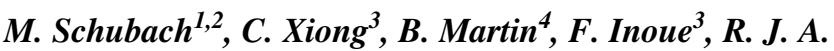
Bell $^{5}$, J. Costello ${ }^{5}$, J. Shendure ${ }^{4}$, N. Ahituv ${ }^{3}$, M. Kircher ${ }^{1,2,4}$

${ }^{1}$ Berlin Institute of Health (BIH), Berlin, Germany, ${ }^{2}$ Charité - Universitätsmedizin Berlin, Berlin, Germany, ${ }^{3}$ Department of Bioengineering and Therapeutic Sciences, University of California San Francisco, San Francisco, CA, United States, ${ }^{4}$ Department of Genome Sciences, University of Washington, Seattle, WA, United States, ${ }^{5}$ Department of Neurosurgery, University of California San Francisco, San Francisco, CA, United States
The majority of variants associated with common diseases and an unknown proportion of causal mutations for rare diseases fall in noncoding regions of the genome. Although catalogs of regulatory elements are steadily improving, we have a limited understanding of the functional effects of mutations within them. Here, we performed saturation mutagenesis in conjunction with massively parallel reporter assays (MPRAs) on 20 disease-associated gene promoters (e.g. HBGl, LDLR, TERT) and enhancers (e.g. IRF6, $S O R T 1, T C F 7 L 2)$, generating functional measurements for over 30,000 single nucleotide substitutions (SNVs) and deletions.

Across elements, we find that a majority of mutations leads to a reduction in activity (transversions more so than transitions, deletions more so than SNVs), suggesting that transcription factor (TF) binding is more easily lost than gained. Further, the density of putative TF binding sites varies widely between regulatory elements. In the Telomerase reverse transcriptase (TERT) promoter, we identify additional and potentially clinically relevant activating mutations, as well as regions that could be targeted to reduce promoter activity. By combining MPRA with GABPA knockdown, 63 TERT promoter variants show a significant change in promoter activity, highlighting GABPA's role in TERT activation and oncogenesis.

Our data provides a rich compendium for studying these 20 disease-associated elements and characterizes effect sizes of regulatory mutations at an unprecedented scale. Although myriad annotations and integrative scores are available, no score consistently performs well in predicting the measured regulatory effects. Thus, our data (https:// mpra.gs.washington.edu) provides a gold standard for further score developments and supports clinical variant interpretation.

M. Schubach: None. C. Xiong: None. B. Martin: None. F. Inoue: None. R.J.A. Bell: None. J. Costello: None. J. Shendure: None. N. Ahituv: None. M. Kircher: None.

\section{C02.4}

Characterization of $G J B 2$ cis-regulatory elements in the DFNB1 locus

\section{A. Le Nabec ${ }^{1}$, A. Quillévérél, C. Le Maréchal ${ }^{1,2}$, C. Férec ${ }^{1,2}$, S. Moisan ${ }^{1,2}$}

${ }^{1}$ Univ Brest, Inserm, EFS, UMR 1078, GGB, Brest, France, ${ }^{2}$ Laboratoire de Génétique Moléculaire et d'Histocompatibilité - CHU, Brest, France

Three-dimensional chromatin organization plays a key role on gene expression. Gene regulation depends on cisregulatory elements which can interact with gene promoter 
by chromatin loop. Alteration of chromatin architecture and/ or cis-acting elements can lead to cis-ruption disorder. Numerous unelucidated nonsyndromic hearing loss and deafness 1 (DFNB1) cases carrying out only one heterozygous pathogenic mutation on Gap Junction Beta 2 (GJB2) gene, led to strongly suggest the presence of distant cisregulation. To analyze chromatin conformation of a large DFNB1 locus, we performed the 5C technology and showed several chromatin contacts with GJB2 promoter. Then, to identify potential regulatory elements of the GJB2 gene, we analysed these chromatin interacting regions with the GJB2 promoter by reporter test activity and identifying cisregulatory elements which have enhancer action and silencer effect on $G J B 2$ expression. Moreover, to explore DFNB1 locus architecture we analysed CTCF (CCCTCbinding factor) binding along $D F N B 1$ locus by ChIP-qPCR and defined an active domain in which cis-acting elements were closer brought to the GJB2 promoter through chromatin looping. This first study of DFNB1 threedimensional chromatin organization allows identification of GJB2 cis-acting elements. Finally, better understand of the molecular mechanisms of DFNB1 deafness could be important to improve genetic diagnosis of hearing loss, to adapt patients care and to develop small therapeutic molecules. This work was supported by grants from the French foundation "La Fondation pour l'Audition", the "Région Bretagne" and the association "Gaétan Salaün".

A. Le Nabec: None. A. Quillévéré: None. C. Le Maréchal: None. C. Férec: None. S. Moisan: None.

\section{C02.5}

\section{Novel insights into molecular mechanisms in X-linked} dystonia-parkinsonism (XDP)

\section{J. Pozojevic ${ }^{1}$, A. Westenberger ${ }^{2}$, C. J. Reyes ${ }^{2}$, K. Grïtz ${ }^{2}$, H. Kirchner ${ }^{3}$, C. Klein ${ }^{2}$, F. J. Kaiser ${ }^{1}$}

${ }^{1}$ Section for Functional Genetics, Institute of Human Genetics, Lübeck, Germany, ${ }^{2}$ Institute for Neurogenetics, Lübeck, Germany, ${ }^{3}$ Department of Internal Medicine I, Lübeck, Germany

$\mathrm{X}$-linked dystonia-parkinsonism (XDP) is a neurodegenerative movement disorder caused by an intronic SINE-VNTRAlu (SVA) retrotransposon insertion in the TAFl gene. Moreover, the number of hexanucleotide repeats within the SVA varies between patients and was recently found to inversely correlate with age at disease onset. TAF1 expression is decreased in XDP patients, and it was recently shown that by excising the SVA from XDP-model cells $T A F 1$ expression levels restore to levels found in wild type cells.
To investigate molecular mechanisms by which the SVA affects TAF1 expression, we used chromatin immunoprecipitation (ChIP) analyzing a set of epigenetic markers within the TAF1 gene region, followed by quantitative PCR (qPCR) in induced pluripotent stem cells (iPSC) from XDP patients and sex-matched controls. Thereby, we identified decreased $\mathrm{H} 3 \mathrm{~K} 4 \mathrm{me} 1$ signal within a set of defined enhancer regions in XDP patients compared to controls, indicating reduced activity of these regulatory regions. Moreover, general acetylation levels of histone 3, a marker of active chromatin, was shown to be decreased within exonic TAF1 regions in patient-derived cell lines. Additional enhancer-promoter reporter assays revealed SVA-mediated alterations of TAF1 promoter activity, even modulated by the number of hexanucleotide repeats within the SVA.

In summary, we used patient-derived cellular models and in vitro reporter gene assays to show SVA-mediated alterations of epigenetic markers within the coding as well as regulatory regions of $T A F 1$. Our results provide new insights into disease-relevant mechanisms of SVA-mediated alterations in TAF1 expression.

Grant reference: German Research Foundation (DFG) Research Unit FOR2488,ProtectMove“

J. Pozojevic: None. A. Westenberger: None. C.J. Reyes: None. K. Grütz: None. H. Kirchner: None. C. Klein: None. F.J. Kaiser: None.

\section{C02.6}

Understanding the roles and the regulation of the Mowat-Wilson Syndrome transcription factor ZEB2 during development and disease

J. Birkhoff ${ }^{1}$, A. Korporaal ${ }^{1}$, S. G. Caraffi ${ }^{2}$, I. Ivanovski ${ }^{2}$, S. Garcia-Minaur ${ }^{3}$, P. Kolovos ${ }^{4}$, R. Brouwer ${ }^{1}$, W. van Ijcken $^{1}$, L. Garavelli' ${ }^{2}$ D. Huylebroeck ${ }^{I}$, A. Conidi ${ }^{1}$

${ }^{1}$ Erasmus MC, Rotterdam, Netherlands, ${ }^{2}$ IRCSS Reggio Emilia, Reggio Emilia, Italy, ${ }^{3}$ La Paz Hospital, Madrid, Spain, ${ }^{4}$ BRIC Institute, Copenhagen, Denmark

Mowat-Wilson Syndrome (MOWS, OMIM \#235730) is a rare intellectual disability (ID) syndrome, often associated with epilepsy, Hirschsprung disease (HD) and multiple congenital anomalies including agenesis of the corpus callosum, congenital heart and eye defects. It is caused by ZEB2 haploinsuficiency, and the degree of syndrome severity is directly correlated with the type of ZEB2 variants: from $Z E B 2$ locus deletions associated with severe phenotypes, to missense mutations that might result in residual ZEB2 protein functions, accompanying milder MOWS. Zeb2 has been extensively studied in conditional knockout mouse embryos in different cell types and is primarily known as transcriptional repressor with an anti- 
BMP, anti-Wnt, and anti-Notch activity in cell differentiation and maturation, including in the central and peripheral nervous systems. In collaborative efforts with clinical geneticists, we have documented the clinical phenotype (ID, epilepsy, HD, developmental milestones), and the causing ZEB2 mutations in a cohort of 82 MOWS patients. We followed up interesting $Z E B 2$ variants associated with mild MOWS and performed RNA-sequencing on both WT and MOWS iPSCs to identify altered genes signature. We have characterized active and repressive enhancers during differentiation within the $3,5 \mathrm{Mb}$ gene desert located upstream of ZEB2. We performed targeted chromatin conformation capture technique to characterize the locus dynamics during differentiation. Deletion of subregions within this gene desert results in similar effects (cell differentiation, target genes) to those we have previously described when differentiating Zeb2 KO mESCs. These findings will contribute to elucidate $Z E B 2$ regulation and its role in MOWS, and the common and patients' specific clinical presentations.

J. Birkhoff: None. A. Korporaal: None. S.G. Caraffi: None. I. Ivanovski: None. S. Garcia-Minaur: None. P. Kolovos: None. R. Brouwer: None. W. van Ijcken: None. L. Garavelli: None. D. Huylebroeck: None. A. Conidi: None.

\section{C02.6}

Understanding the roles and the regulation of the Mowat-Wilson Syndrome transcription factor ZEB2 during development and disease

J. Birkhoff', A. Korporaal', S. G. Caraffi, I. Ivanovski', S. Garcia-Minaur ${ }^{3}$, P. Kolovos ${ }^{4}$, R. Brouwer ${ }^{1}$, W. van Ijcken $^{1}$, L. Garavelli ${ }^{2}$, D. Huylebroeck ${ }^{1}$, A. Conidi ${ }^{1}$

${ }^{1}$ Erasmus MC, Rotterdam, Netherlands, ${ }^{2}$ IRCSS Reggio Emilia, Reggio Emilia, Italy, ${ }^{3}$ La Paz Hospital, Madrid, Spain, ${ }^{4}$ BRIC Institute, Copenhagen, Denmark

Mowat-Wilson Syndrome (MOWS, OMIM \#235730) is a rare intellectual disability (ID) syndrome, often associated with epilepsy, Hirschsprung disease (HD) and multiple congenital anomalies including agenesis of the corpus callosum, congenital heart and eye defects. It is caused by ZEB2 haploinsuficiency, and the degree of syndrome severity is directly correlated with the type of $Z E B 2$ variants: from $Z E B 2$ locus deletions associated with severe phenotypes, to missense mutations that might result in residual ZEB2 protein functions, accompanying milder MOWS. Zeb2 has been extensively studied in conditional knockout mouse embryos in different cell types and is primarily known as transcriptional repressor with an antiBMP, anti-Wnt, and anti-Notch activity in cell differentiation and maturation, including in the central and peripheral nervous systems. In collaborative efforts with clinical geneticists, we have documented the clinical phenotype (ID, epilepsy, HD, developmental milestones), and the causing $Z E B 2$ mutations in a cohort of 82 MOWS patients. We followed up interesting ZEB2 variants associated with mild MOWS and performed RNAsequencing on both WT and MOWS iPSCs to identify altered genes signature. We have characterized active and repressive enhancers during differentiation within the $3,5 \mathrm{Mb}$ gene desert located upstream of ZEB2. We performed targeted chromatin conformation capture technique to characterize the locus dynamics during differentiation. Deletion of subregions within this gene desert results in similar effects (cell differentiation, target genes) to those we have previously described when differentiating Zeb2 KO mESCs. These findings will contribute to elucidate $Z E B 2$ regulation and its role in MOWS, and the common and patients' specific clinical presentations.

J. Birkhoff: None. A. Korporaal: None. S.G. Caraffi: None. I. Ivanovski: None. S. Garcia-Minaur: None. P. Kolovos: None. R. Brouwer: None. W. van Ijcken: None. L. Garavelli: None. D. Huylebroeck: None. A. Conidi: None.

C03

Neurogenetic and psychiatric disorders

C03.1

Intronic expansions of an ATTTC pentamer in the STARD7 gene underlie Familial Adult Myoclonic Epilepsy linked to chromosome 2 (FAME2)

J. Gecz', Z. Afawi', M. Bahlo ${ }^{3}$, M. F. Bennett ${ }^{3}$, S. F. Berkovic ${ }^{4}$, F. Bisulli, F. Brancati ${ }^{6}$, L. Canafoglia ${ }^{7}$, G. Casari ${ }^{8,9}$, R. van Coller ${ }^{10}$, M. A. Corbett ${ }^{1}$, D. Crompton $^{11}$, C. Depienne ${ }^{12}$, R. Guerrini ${ }^{13}$, E. Hirsch ${ }^{14}$, M. Klein ${ }^{15}$, L. Licchetta ${ }^{5}$, A. van den Maagdenberg ${ }^{16}$, D. $\mathrm{Mei}^{13}$, T. Pippucci ${ }^{17}$, L. Sadleir ${ }^{18}$, I. E. Scheffer ${ }^{19}$, S. M. Sisodiya ${ }^{20}$, P. Striano ${ }^{21}$, A. Suppa ${ }^{22,23}$, M. A. Tijssen ${ }^{24}$, L. Veneziano ${ }^{25}$, F. Zara ${ }^{26}$, The FAME Consortium

${ }^{1}$ The University of Adelaide, Adelaide, Australia, ${ }^{2}$ Tel Aviv University, Tel Aviv, Israel, ${ }^{3}$ The Walter and Eliza Hall Institute of Medical Research, Melbourne, Australia, ${ }^{4}$ University of Melbourne, Melbourne, Australia, ${ }^{5}$ IRCCS Institute of Neurological Sciences, Bologna, Italy, ${ }^{6}$ University of L'Aquila, L'Aquila, Italy, ${ }^{7}$ Fondazione IRCCS Istituto Neurologico Carlo Besta, Milano, Italy, ${ }^{8}$ Telethon Institute of Genetics and Medicine, Naples, Italy, ${ }^{9}$ San Raffaele University, Milano, Italy, ${ }^{10}$ University of Pretoria, 
Pretoria, South Africa, ${ }^{11}$ Northern Health, Melbourne, Australia, ${ }^{12}$ Institut für Humangenetik, Universitätsklinikum Essen, Essen, Germany, ${ }^{13}$ Meyer Children's Hospital, Florence, Italy, ${ }^{14}$ Strasbourg University Hospital, Strasbourg, France, ${ }^{15}$ University of Frankfurt, Frankfurt, Germany, ${ }^{16}$ Leiden University Medical Centre, Leiden, Belgium, ${ }^{17}$ Sant'Orsola-Malpighi University Hospital, Bologna, Italy, ${ }^{18}$ University of Otago, Wellington, New Zealand, ${ }^{19}$ University of Melbourne, Melbourne, Italy, ${ }^{20}$ UCL Queen Square Institute of Neurology, London, United Kingdom, ${ }^{21}$ University of Genoa, Genova, Italy, ${ }^{22}$ University of Rome, Rome, Italy, ${ }^{23}$ IRCCS Neuromed Institute, Rome, Italy, ${ }^{24}$ University of Groningen, Groningen, Netherlands, ${ }^{25}$ Institute of Translational Pharmacology, Rome, Italy, ${ }^{26} G$ Gaslini Institute, Genova, Italy

Dynamic mutations are pathogenic expansions of short tandem repeat sequences and are frequently associated with disorders of movement. Familial Adult Myoclonic Epilepsy (FAME) is characterised by cortical myoclonic tremor usually beginning in the second decade of life with later onset of myoclonic and generalised tonic clonic seizures. There have been at least four different FAME loci identified through linkage analysis of large autosomal dominant families that are located on chr2, 3, 5 and 8. Expansion of an endogenous ATTTT pentamer coupled with insertion and expansion of a novel ATTTC pentamer within the SAMD12 gene are the cause of chr8 FAME (FAME1). Using both long (Oxford Nanopore and PacBio) and short (Illumina and Complete Genomics) read whole genome sequencing, repeat primed PCR and Southern blotting we show that chr2 FAME is caused by a similar dynamic mutation of the same pentanucleotide repeats that arise in the first intron of the STARD7 gene. The ATTTC repeat expansion segregates with FAME in over 250 individuals from all 22 families tested so far. RNA-Seq from patient derived fibroblast cell lines showed no accumulation of the AUUUU or AUUUC repeat sequences, however a significant enrichment of differentially expressed genes implicated in lysosomal storage disorders and progressive myoclonic epilepsy suggested that molecular pathways involved in cellular waste collection were active. Our data demonstrate the role of ATTTC repeat expansion in FAME2 and strongly support, regardless of the genomic locus, ATTTC pentamer expansions as a general cause of FAME, which affect thousands of individuals worldwide.

J. Gecz: None. Z. Afawi: None. M. Bahlo: None. M.F. Bennett: None. S.F. Berkovic: None. F. Bisulli: None. F. Brancati: None. L. Canafoglia: None. G. Casari: None. R. van Coller: None. M.A. Corbett: None. D. Crompton: None. C. Depienne: None. R. Guerrini: None. E. Hirsch: None. M. Klein: None. L. Licchetta: None. A. van den Maagdenberg: None. D. Mei: None. T. Pippucci: None.
L. Sadleir: None. I.E. Scheffer: None. S.M. Sisodiya: None. P. Striano: None. A. Suppa: None. M.A. Tijssen: None. L. Veneziano: None. F. Zara: None.

C03.2

SINEUPs technology: a new route to possibly treat haploinsufficiency-induced Epilepsy and Autism Spectrum Disorders (ASDs)

\section{Arnoldi ${ }^{I}$, F. Di Leva', G. L. Carvill', S. Zucchelli, S. Gustincich ${ }^{4}$, M. Biagioli $^{1}$}

${ }^{I}$ Centre for Integrative Biology, University of Trento, Trento, Italy, ${ }^{2}$ Feinberg School of Medicine, Northwestern University, Chicago, IL, United States, ${ }^{3}$ Department of Health Sciences, Università del Piemonte Orientale, Novara, Italy, ${ }^{4}$ Department of Neuroscience and Brain Technologies, Istituto Italiano di Tecnologia (IIT), Genova, Italy

Introduction: Recurrent mutations in chromodomain helicase DNA-binding protein 2 and 8 (CHD2 and CHD8), functioning in chromatin regulation and transcription, are emerging as prominent risk factors for Epilepsy and ASD, respectively. Most of the mutations in these genes are disruptive, leading to haploinsufficiency. Thus, any molecular manipulation eliciting an increase in CHD2/CHD8 proteins, could prove beneficial for therapeutic development. Here, we intended to provide a Proof-of-Principle of how SINEUP - recently described class of non-coding RNAs able to augment, in a specific and controlled way, the expression of target proteins - can rise the translation of CHD2/CHD8 proteins and possibly rescue haploinsufficiency-associated phenotypes.

Methods: We designed and cloned SINEUP targeting human $C H D 2 / C H D 8$ isoforms and tested their efficacy to increase CHD2/CHD8 protein translation in human iPSderived neuronal progenitors (iPS-NPC) expressing normal and reduced levels of the target proteins and in patients' fibroblasts bearing CHD2/CHD8 heterozygous loss-offunction mutations.

Results: While different $\mathrm{CHD} 2 / \mathrm{CHD} 8$-SINEUP molecules were not able to significantly increase protein level in parental iPS-NPC, expressing physiologic levels of CHD2/ CHD8, SINEUP revealed to be effective in upregulating CHD2/CHD8 protein translation when reduced levels of the target proteins were expressed. The efficacy of CHD2/ CHD8 SINEUP is currently tested on patient derived cell lines harbouring $C H D 2 / C H D 8$ truncating mutations.

Conclusion: Our data represent an initial Proof-ofConcept that SINEUP, RNA-based approach could reveal functional to rescue $C H D 2 / C H D 8$ haploinsufficiency with 
important implications for therapeutic development in Epilepsy and ASD.

Project sponsored by CURE Epilepsy and Brain \& Behaviour foundations.

M. Arnoldi: None. F. Di Leva: None. G.L. Carvill: None. S. Zucchelli: None. S. Gustincich: None. M. Biagioli: None.

\section{C03.3}

Brain somatic mutations associated with aging contribute to dysregulation of Tau phosphorylation in Alzheimer's disease

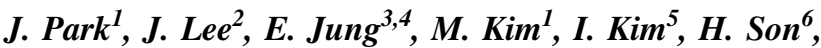
S. $\mathrm{Kim}^{6}$, S. Kim ${ }^{7}$, Y. Park ${ }^{8}$, I. Mook-Jung ${ }^{3,4}, \mathrm{~S} . \mathrm{Yu}^{2}$, J. Lee $^{1,5}$

${ }^{1}$ Biomedical Science and Engineering Interdisciplinary Program, Korea Advanced Institute of Science and Technology (KAIST), Daejeon, Korea, Republic of, ${ }^{2}$ Center for Computational Science Platform, Division of National Supercomputing, Korea Institute of Science and Technology Information, Daejeon, Korea, Republic of, ${ }^{3}$ Department of Biochemistry \& Biomedical Sciences, College of Medicine, Seoul National University, Seoul, Korea, Republic of, ${ }^{4}$ Neuroscience Research Institute, College of Medicine, Seoul National University, Seoul, Korea, Republic of, ${ }^{5}$ Graduate School of Medical Science and Engineering, Korea Advanced Institute of Science and Technology (KAIST), Daejeon, Korea, Republic of, ${ }^{6}$ Department of Biomedical Systems Informatics, Brain Korea 21 PLUS Project for Medical Science, Yonsei University College of Medicine, Seoul, Korea, Republic of, ${ }^{7}$ Stanley Medical Research Institute (SMRI), Laboratory of Brain Research, Daejeon, MD, United States, ${ }^{8}$ Center for Cognition and Sociality, Institute for Basic Science (IBS), Daejeon, Korea, Republic of

Brain somatic mutations arising from the neural stem cell niche appear to underlie several neurodevelopmental disorders and brain tumors. Studies, however, have yet to shed light on the significance and pathogenic roles of brain somatic mutations in Alzheimer's disease (AD). Here, we performed deep whole-exome sequencing (average read depth 584X) in 111 postmortem hippocampal formation and matched blood tissues from 52 AD patients and 11 nondemented individuals. We found an average of 11.96 and 12.55 somatic single nucleotide variations (SNVs) in brain tissue from $\mathrm{AD}$ patients and controls, respectively, and 59.31 and 59.17 SNVs from blood. The number of somatic mutations in brain specimens increased significantly with aging, and the rate of mutation accumulation in the brain was 4.8 -fold slower than that in blood. The pathogenic somatic mutations identified in $26.9 \%$ (14 of 52) of $\mathrm{AD}$ individuals were enriched in PI3K-AKT, MAPK, and AMPK pathway genes known to contribute to hyperphosphorylation of Tau. Further, we discovered that a pathogenic brain somatic mutation in PIN1 was more abundant in AT8-positive neurons in the entorhinal cortex and leads to a loss of function mutation. In vitro mimicking of haploinsufficiency of PIN1 aberrantly increased the phosphorylation and aggregated form of Tau. Altogether, this study provides new insights into the genetic architecture underlying the pathogenesis of $\mathrm{AD}$, demonstrating that brain somatic mutations associated with aging contribute to the initial appearance of Tau pathology in the hippocampal formation of the $\mathrm{AD}$ brain.

J. Park: None. J. Lee: None. E. Jung: None. M. Kim: None. I. Kim: None. H. Son: None. S. Kim: None. S. Kim: None. Y. Park: None. I. Mook-Jung: None. S. Yu: None. J. Lee: None.

\section{C03.4}

Loss of neutral sphingomyelinase-3 (SMPD4) links neurodevelopmental disorders to cell cycle and nuclear envelope anomalies
D. J. Smits ${ }^{1}$, P. Magini ${ }^{2}$, L. Vandervore ${ }^{3,1}$, R. Schot ${ }^{\text {, }}$, M. Columbaro ${ }^{2}$, E. Kasteleijn ${ }^{1}$, M. van der Ent ${ }^{4}$, F. Palombo ${ }^{2}$, L. Iommarini, M. H. Lequin', A. M. Porcelli, P. Govaert ${ }^{8}$, M. Dremmen ${ }^{9}$, M. C. Y. de Wit $^{10}$, M. Severino ${ }^{11}$, M. T. Divizia ${ }^{12}$, N. Ordonez- Herrera $^{13}$, A. Alhashem ${ }^{14}$, A. Al Fares ${ }^{14}$, M. Al Ghamdi ${ }^{14}$, M. Al Ghamdi' ${ }^{14}$, A. Rolfs ${ }^{13}$, P. Bauer ${ }^{13}$, J. Demmers ${ }^{15}$, F. Verheijen ${ }^{1}, M$. Wilke ${ }^{1}$, M. van Slegtenhorst ${ }^{l}$, P. van der Spek $^{16}$, A. Jansen ${ }^{3}$, R. Stottmann ${ }^{17}$, R. Hufnagel ${ }^{17}$,
R. Hopkin ${ }^{17}$, D. Aljeaid ${ }^{17}$, W. Wiszniewski ${ }^{18}$,
P. Gawlinski ${ }^{18}$, W. B. Dobyns ${ }^{18}$, M. Seri' ${ }^{2}$, T. Pippucci ${ }^{2}$, M. Fornerod ${ }^{4}$, G. M. S. Mancini ${ }^{I}$

${ }^{1}$ Department of Clinical Genetics, Erasmus University Medical Center, Rotterdam, Netherlands, ${ }^{2}$ Medical Genetics Unit, University of Bologna, Bologna, Italy, ${ }^{3}$ Neurogenetics research group, Vrije Universiteit Brussel, Brussel, Belgium, ${ }^{4}$ Department of Cell Biology, Erasmus University Medical Center, Rotterdam, Netherlands, ${ }^{5}$ University of Bologna, Bologna, Italy, ${ }^{6}$ University Medical Center Utrecht, Utrecht, Netherlands, ${ }^{7}$ Department of Pharmacy and Biotechnologies, University of Bologna, Bologna, Italy, ${ }^{8}$ Department of Pediatrics, division of neonatology, Erasmus University Medical Center, Rotterdam, Netherlands, ${ }^{9}$ Department of Radiology, Erasmus University Medical Center, Rotterdam, Netherlands, ${ }^{10}$ Department of Neurology, Erasmus University Medical Center, Rotterdam, Netherlands, ${ }^{11}$ Neuroradiology department, Istituto Giannina Gaslini, Genoa, Italy, ${ }^{12}$ Medical Genetics Unit, Istituto 
Giannina Gaslini, Genoa, Italy, ${ }^{13}$ Centogene AG, Rostock, Germany, ${ }^{14}$ Department of Genetics, King Faisal Specialist Hospital and Research Center, Riyadh, Saudi Arabia, ${ }^{15}$ Department of Molecular Genetics, Proteomics center, Erasmus University Medical Center, Rotterdam, Netherlands, ${ }^{16}$ Department of Bioinformatics, Erasmus University Medical Center, Rotterdam, Netherlands, ${ }^{17}$ Division of Human Genetics, Cincinnati Children's Hospital Medical Center, University of Cincinnati College of Medicine, Cincinnati, OH, United States, ${ }^{18}$ Department of Molecular and Medical Genetics, Oregon Health and Science University, Portland, OR, United States

Introduction: Several neurodevelopmental processes including neuronal survival, migration and differentiation are controlled by sphingolipid metabolism. Sphingomyelinases generate ceramide from sphingomyelin as a second messenger in intracellular signaling pathways involved in cell proliferation, differentiation, or apoptosis. While the role of acid sphingomyelinase is well established, the role of neutral sphingomyelinases in human neurodevelopment has remained elusive.

Methods: Twenty-five children from ten unrelated families presented with microcephaly with simplified gyral pattern, cerebellar hypoplasia, severe developmental encephalopathy, congenital arthrogryposis, diabetes mellitus and early demise. All index cases have bi-allelic loss of function variants in the SMPD4 gene, coding for neutral sphingomyelinase-3.We performed additional studies to elucidate the mechanisms contributing to disease pathogenesis.

Results: Fibroblasts from affected individuals showed morphologic endoplasmic reticulum (ER) cisternae abnormalities, consistent with a previously suggested function of SMPD4 in the ER. Overexpression of human Myc-tagged SMPD4 in HEK392T cells showed localization of the protein to both the nuclear envelope and the ER. Previous studies localized SMPD4 to the outer nuclear membrane. Mass spectrometry of SMPD4-associated proteins detected peptides belonging to nuclear pore complex proteins. We also observed delayed cell cycle progression after downregulation of SMPD4 by siRNA. These data are similar to former studies in HeLa cells showing mitotic abnormalities after siSMPD4 treatment.

Conclusion: Our study describes a novel microcephaly syndrome characterized by severe neurologic abnormalities. Our results suggest a major function of neutral sphingomyelinase- 3 in regulation of mitosis through modulation of nuclear membrane lipids, pointing to a novel mechanism and pathway in the pathogenesis of microcephaly.

D.J. Smits: None. P. Magini: None. L. Vandervore: None. R. Schot: None. M. Columbaro: None. E.
Kasteleijn: None. M. van der Ent: None. F. Palombo: None. L. Iommarini: None. M.H. Lequin: None. A.M. Porcelli: None. P. Govaert: None. M. Dremmen: None. M.C.Y. de Wit: None. M. Severino: None. M.T. Divizia: None. N. Ordonez-Herrera: None. A. Alhashem: None. A. Al Fares: None. M. Al Ghamdi: None. M. Al Ghamdi: None. A. Rolfs: None. P. Bauer: None. J. Demmers: None. F. Verheijen: None. M. Wilke: None. M. van Slegtenhorst: None. P. van der Spek: None. A. Jansen: None. R. Stottmann: None. R. Hufnagel: None. R. Hopkin: None. D. Aljeaid: None. W. Wiszniewski: None. P. Gawlinski: None. W.B. Dobyns: None. M. Seri: None. T. Pippucci: None. M. Fornerod: None. G.M.S. Mancini: None.

\section{C03.5}

Exploring the impact of CHD2 mutations on DNA double strand break (DSB) repair via non-homologous end joining (NHEJ) using Cas9 and Nanopore sequencing in human induced pluripotent stem cells (hIPSC)

\section{Tully, M. Alsaqati, A. Baldwin, W. Plumbly,}

\section{A. Harwood}

Neuroscience and Mental Health Research Institute, Cardiff, United Kingdom

Introduction: Heterozygous CHD2 mutations cause early onset epileptic. The mechanism by which this occurs is not understood, however previous evidence suggests a role for CHD2 mediated chromatin remodelling during NHEJ.

Methods: hIPSCs with doxycycline inducible Cas9 expression were used to create a heterozygous CHD2 mutant with an $11 \mathrm{bp}$ frameshift mutation (CHD2+/-). To study DSB repair, five genomic targets were chosen, and three gRNAs lipofected for each, in wild type (WT) and CHD2+/- cell cultures. In order to capture a wide range of indels, a $1 \mathrm{~kb}$ region was amplified around each target using PCR - with a second used to apply DNA barcodes to each sample. Ultra-deep whole-molecule sequencing was performed using the oxford nanopore minion. A comparison was made between the indels seen in the $\mathrm{CHD} 2+/-$ and WT cell lines.

Results: A total of $4 \times 10^{6}$ reads were obtained. In all targets, CHD2 mutations resulted in statistically significant increase in the number of larger (40bp-500bp) deletions, related to simultaneous cutting with more than one gRNA. Depending on the target, the increase ranged between 2-fold $\left(\mathrm{p}=1.48 \times 10^{-22}\right)$ and 27.1 -fold $\left(\mathrm{p}=\mathrm{x} 10^{-260}\right)$. At 3 of 5 targets, there was also a discernible shift in the pattern of smaller indels ( $<50 \mathrm{bp}$ length) at each individual cut site. 
Conclusion: The increase in mutations caused by multiple cuts suggests that heterozygous mutations alter the dynamics of DNA repair, causing cuts to persist longer. In the developing brain, this could increase the risk of translocations occurring, leading to a potential increase in pathological mosaicism in the mature brain.

I. Tully: None. M. Alsaqati: None. A. Baldwin: None. W. Plumbly: None. A. Harwood: None.

\section{C03.6}

De-novo mutations in TAOK1 cause neurodevelopmental disorders

M. Dulovic Mahlow ${ }^{1}$, J. Trinh ${ }^{1}$, K. Kumar Kandaswamy ${ }^{2}$, G. Julius Braathen ${ }^{3}$, N. Di Donato ${ }^{4}$, M. Werber ${ }^{2}$,

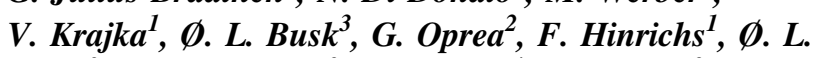
Holla $^{3}$, M. E.R. Weiss ${ }^{2}$, A. Kahlert ${ }^{4}$, S. Kishore ${ }^{2}$, K. Tveten ${ }^{3}, M . \operatorname{Vos}^{1}$, A. Rolfs ${ }^{5,2}, K$. Lohmann ${ }^{1}$

${ }^{1}$ Institute of Neurogenetics, Luebeck, Germany, ${ }^{2}$ Centogene AG, Rostock, Germany, ${ }^{3}$ Department of Medical Genetics, Telemark Hospital Trust, Skien, Norway, ${ }^{4}$ Institute of Clinical Genetics, Technical University of Dresden, Dresden, Germany, ${ }^{5}$ University of Rostock, Rostock, Germany

Introduction: De-novo mutations represent a significant cause of neurodevelopmental disorders (NDD) such as neurodevelopmental delay and intellectual disability. However, only about 50\% of the cases can be genetically explained.

Methods: We identified de-novo variants in the TAOKI (thousand and one amino acid kinase 1) gene in NDD patients. To validate this potential novel disease gene we (1) used GeneMatcher to identify additional patients, (2) established a primary fibroblast line of one of the patients and collected RNA from patient's fibroblasts and blood (PAXTube), and (3) established an RNAi-mediated knockdown Drosophila model.

Results: We compared the frequency of de-novo variants in patient-parent trios with $(\mathrm{n}=2,030)$ vs. without $(\mathrm{n}=$ $2,755)$ NDD. We identified de-novo variants in TAOK1 in four NDD patients but not in non-NDD patients. Two additional patients were found through GeneMatcher. All variants were absent from gnomAD. The patients shared a common phenotype of developmental delay. Additionally, four patients had muscular hypotonia and three autism. In the fibroblast culture, we demonstrated highly reduced TAOK1 mRNA levels compatible with nonsense-mediated mRNA decay. No detectable phosphorylated TAOK1 protein was found in these cells. Knockdown of TAO (CG14217) in Drosophila resulted in developmental delay and reduced survival compared to control flies. Immunostaining of the neuromuscular junctions (NMJs), their endings (boutons), and the ventral nerve cord (VNC) revealed changed NMJ and VNC morphology and significantly decreased amount of boutons compared to control flies.

Conclusion: We provide compelling evidence that denovo mutations in TAOK1 are causative for a novel form of NDD.

M. Dulovic Mahlow: None. J. Trinh: None. K. Kumar Kandaswamy: None. G. Julius Braathen: None. N. Di Donato: None. M. Werber: None. V. Krajka: None. Ø. L. Busk: None. G. Oprea: None. F. Hinrichs: None. Ø. L. Holla: None. M. E.R. Weiss: None. A. Kahlert: None. S. Kishore: None. K. Tveten: None. M. Vos: None. A. Rolfs: None. K. Lohmann: None.

\section{C04 \\ Fertility}

\section{C04.1}

Ectopic expression of CGG repeats leads to impaired response to gonadotropin hormones and reduced fertility with age in a mouse model of the FMR1 premutation

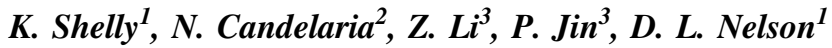 \\ ${ }^{I}$ Duncan Neurological Research Institute, Houston, TX, United States, ${ }^{2}$ Baylor College of Medicine, Houston, TX, United States, ${ }^{3}$ Emory University School of Medicine, Atlanta, GA, United States}

Carriers of expanded CGG repeats in the 5'UTR of $F M R l$ are at risk for Fragile $\mathrm{X}$-associated Primary Ovarian Insufficiency (FXPOI). Two mouse lines expressing either CGG RNA-only (RNA-only) or CGG RNA and its translated polyglycine product FMRpolyG (FMRpolyG +RNA) were used to assess fertility using breeding and superovulation studies in order to model FXPOI. Phenotypic data were collected longitudinally, and ovarian histology was examined. CGG RNA and FMRpolyG +RNA both contribute to ovarian dysfunction, albeit differently. Immunostaining shows FMRpolyG is present in oocytes and granulosa in early postnatal life and gonadotropin stimulation reveals that young RNA-only and FMRpolyG + RNA mice ovulate fewer oocytes/female. Ovaries during ovulation ( $8 \mathrm{~h}$ post-hCG administration) show impaired cumulus expansion in the preovulatory follicles of RNA-only and FMRpolyG+RNA ovaries. With age, FMRpolyG+RNA mice exhibit declining fertility, but this reduction was not seen in RNA-only mice. Cessation of breeding in FMRpolyG+RNA females is preceded by significant weight gain compared to control mice, and 
histology shows a lack of ovulation as well as hyperplastic stroma and disorganized theca in aged ovaries. An increased incidence of cysts present in both groups of CGGexpressing ovaries suggest altered steroidogenesis and is confirmed by qRT-PCR from whole ovary tissue collected from aged mice. Globally expressed FMRpolyG+CGG RNA leads to anovulation, altered steroidogenic profile, and fertility decline with age, while CGG RNA-only mice do not exhibit a decline in fertility or increased weight but diminished response to superovulation and cumulus expansion defects similar to the FMRpolyG+RNA females suggest that CGG RNA-alone can affect ovarian function.

K. Shelly: None. N. Candelaria: None. Z. Li: None. P. Jin: None. D.L. Nelson: None.

\section{C04.2}

Proteomics and single-cell RNA analysis of Akap4knockout mice model confirm indispensable role of Akap4 in spermatogenesis

N. $\mathrm{Li}^{1}, \mathrm{X} . \mathrm{Fang}^{1}$, L. Huang ${ }^{1,2}, J . \mathrm{Xu}^{3}, \mathrm{C} . \mathrm{Ma}^{3}$, Z. Chen ${ }^{3}$, Z. Zhang ${ }^{1}$, C. Liao ${ }^{1}$, S. Zheng ${ }^{1}$, P. Huang ${ }^{4,2}$, W. Xu ${ }^{5}$, L. Sun ${ }^{3}$

${ }^{1}$ Guangzhou Institute of Pediatrics, Guangzhou Women and Children's Medical Center, Guangzhou Medical University, Guangzhou, China, ${ }^{2}$ Department of Urology, Zhujiang Hospital, Southern Medical University, Guangzhou, China, ${ }^{3}$ Center of Reproductive Medicine, Guangzhou Women and Children's Medical Center, Guangzhou Medical University, Guangzhou, China, ${ }^{4}$ Department of Urology, Okayama University Graduate School of Medicine, Dentistry and Pharmaceutical Sciences, Okayama, Japan, ${ }^{5}$ Reproductive Endocrinology and Regulation Laboratory, Key Laboratory of Obstetric, Gynecologic and Pediatric Diseases and Birth Defects of Ministry of Education, West China Second University Hospital, Sichuan University, Chengdu, China

Sperm fibrous sheath, a unique cytoskeletal structure, is implicated in various sperm physiological functions, such as sperm maturation, motility and capacitation. AKAP4 has been described to be required for structural and functional integrity of the fibrous sheath. We generated Akap4knockout mice line using CRISPR-Cas9 system. Cytomorphology and motility of sperms and testes were studied, confirming loss of Akap4 led to abnormal sperm morphology, motility and infertility. The proteomic components of testes were examined and Akap4 was found to be significantly decreased in the Akap4-knockout mice. Testes single-cell RNA sequencing and analysis revealed three genes with significant change in the general cell population, i.e., Akap4, Haspin, and Ccdc38. The single-cell RNA expression profiles also showed that the major difference between Akap4-knockout and wild-type testes existed in the elongating cell cluster, where in the Akap4-knockout testes, a subgroup of elongating cells with maker genes involved in cell adhesion and migration were increased, while a subgroup of elongating cells marked by mitochondrial sheath genes were decreased. Our results revealed the complex and well-coordinated procedures of spermatogenesis, and substantiated Akap4's indispensable roles in the integrity of sperm flagellum and the step-wise maturation of sperms.

N. Li: None. X. Fang: None. L. Huang: None. J. Xu: None. C. Ma: None. Z. Chen: None. Z. Zhang: None. C. Liao: None. S. Zheng: None. P. Huang: None. W. Xu: None. L. Sun: None.

C04.3

Discovery of selfish mutations expanding in the male germline with duplex sequencing

R. Salazar ${ }^{1}$, B. Arbeithuber ${ }^{2}$, M. Ivankovic ${ }^{1}$, G. Povysil ${ }^{3,4}$, M. Heinzl', I. Hartl', S. Moura', J. Pröll ${ }^{5}$, I. TiemannBoege $^{I}$

${ }^{1}$ Institute of Biophysics, Johannes Kepler University, Linz, Austria, ${ }^{2}$ Department of Biology, Penn State University, State College, PA, United States, ${ }^{3}$ Institute of Bioinformatics, Johannes Kepler University, Linz, Austria, ${ }^{4}$ Institute for Genomic Medicine, Columbia University Irving Medical Center, New York, NY, United States, ${ }^{5}$ Center for Medical Research, Faculty of Medicine, Johannes Kepler University, Linz, Austria

Introduction: De novo mutations (DNM) are an important player in heritable diseases and evolution, yet little is known about the different mutagenic processes in our germline. Of interest are a few highly recurrent DNM associated with congenital disorders and/or rasopathies, that have been described as selfish mutations expanding in the male germline. We have adapted an ultra-sensitive sequencing (USS) technology based on duplex-barcoding to distinguish both DNA strands, thus increasing the sensitivity to one mutation in $10^{7}$ sequenced bases. This technology was applied to study the expansion of selfish mutations in the FGFR3 gene in the male germline.

Materials and Methods: A 3000bp coding region of the FGFR3 gene including the transmembrane and the tyrosine kinase domains was sequenced with USS in two DNA sperm pools from an old and young donor group.

Results: DNM with frequencies of $10^{-3}-10^{-5}$ were identified in 41 exonic positions, several of which have been described in congenital disorders and/or cancer. The achondroplasia associated mutation c.1138G $>$ A was detected at similar frequencies as the reported occurrence. 
Also, various substitutions are viable and have been reported in population data (gnomAD), albeit at lower frequencies. Mutations found in both pools show higher frequencies in older donors.

Conclusion: Our USS method has proven to be successful in detecting low frequency DNM. Also, it is an important strategy to identify driver mutations in the male germline expanding with paternal age with potentially important health consequences.

Project funded by the Linz Institute of Technology (LIT213201001) and FWF (FWF308667000).

R. Salazar: None. B. Arbeithuber: None. M. Ivankovic: None. G. Povysil: None. M. Heinzl: None. I. Hartl: None. S. Moura: None. J. Pröll: None. I. TiemannBoege: None.

\section{C04.4}

Exome sequencing reveals de novo mutations and deletions in severe idiopathic male infertility

M. S. Oud ${ }^{1}$, R. M. Smits ${ }^{2}$, F. K. Mastrorosa ${ }^{3}$, H. Smith ${ }^{3}$, M. J. Xavier ${ }^{3}$, G. S. Holt ${ }^{3}$ H. Sheth ${ }^{3}$, B. J. Houston ${ }^{4}$, M. K. O'Bryan ${ }^{4}$, P. F. de Vries $^{1}$, B. Alobaidi ${ }^{3}$, H. Ismail ${ }^{3}$, A. Garcia-Rodriguez ${ }^{3}$, A. Mikulasova ${ }^{5}$, C. Gilissen ${ }^{1}$, L. E. L. M. Vissers ${ }^{1}$, F. Tüttelmann ${ }^{6}$, K. McEleny ${ }^{7}$, J. Coxhead $^{8}$, S. Cockell', D. D. M. Braat ${ }^{2}$, K. Fleischer $^{2}$, G. W. van der Heijden ${ }^{1,2}$ L. Ramos ${ }^{2}$, J. A. Veltman ${ }^{1,3}$

${ }^{1}$ Department of Human Genetics, Radboudumc, Nijmegen, Netherlands, ${ }^{2}$ Department of Obstetrics and Gynaecology, Radboudumc, Nijmegen, Netherlands, ${ }^{3}$ Institute of Genetic Medicine, Newcastle upon Tyne, United Kingdom, ${ }^{4}$ School of Biological Sciences, Monash University, Melbourne, Australia, ${ }^{5}$ Institute of Cellular Medicine, Newcastle upon Tyne, United Kingdom, ${ }^{6}$ Institute of Human Genetics, Münster, Germany, ${ }^{7}$ Newcastle Fertility Centre, Newcastle upon Tyne, United Kingdom, ${ }^{8}$ Genomics Core Facility, Newcastle University, Newcastle upon Tyne, United Kingdom, ${ }^{9}$ Bioinformatics Support Unit, Newcastle University, Newcastle upon Tyne, United Kingdom

Human reproduction is vital to our species but worldwide infertility affects 1 in 6 couples, of which half of the cases are explained by a male factor. Although thousands of genes are known to be involved in spermatogenesis, the genetic causes behind severe spermatogenic failure remain largely unknown. De novo mutations (DNMs), that arise spontaneously in the germline or postzygotically, are known to play a prominent role in early-onset disorders with reduced fitness. Here we report on the first exome sequencing study to investigate the role of DNMs in male infertility. We examined and sequenced 108 patients suffering from azoospermia or severe oligozoospermia $(<5$ million sperm/ml) and their fertile parents. In total, we identified and validated 92 protein-altering DNMs, which show an enrichment in protein network edges. Also, we found an enrichment of loss-of-function (LoF) variants in extremely LoF intolerant genes. Of all DNMs, 22 are likely to disrupt normal gene function and lie in genes involved in sperm production such as TOPAZ1 and ODF1. Complementarily, we detected de novo copy number variants in 2 patients affecting multiple genes involved in cell replication and gamete production. Currently, we are performing replication studies in additional cohorts to identify recurrently mutated genes. Additionally, detailed phenotypic reevaluation of patient phenotype, immunofluorescence stainings of patient material and genetic screens using Drosophila Melanogaster are being performed to more confidently link genes affected by a DNM to male infertility. Our data provide the first indications that DNMs may play an important role in severe male infertility.

M.S. Oud: None. R.M. Smits: None. F.K. Mastrorosa: None. H. Smith: None. M.J. Xavier: None. G.S. Holt: None. H. Sheth: None. B.J. Houston: None. M.K. O'Bryan: None. P.F. de Vries: None. B. Alobaidi: None. H. Ismail: None. A. Garcia-Rodriguez: None. A. Mikulasova: None. C. Gilissen: None. L.E.L.M. Vissers: None. F. Tüttelmann: None. K. McEleny: None. J. Coxhead: None. S. Cockell: None. D.D.M. Braat: None. K. Fleischer: None. G.W. van der Heijden: None. L. Ramos: None. J.A. Veltman: None.

\section{C04.5}

CETN1 is associated with spermatogenesis and male fertility: Genetic and functional perspectives

D. V. S. Sudhakar', S. Jaishankar ${ }^{2}$, R. Phanindranath', A. Pawar', V. Jayaraman ${ }^{3}$, D. Verma ${ }^{4}$, J. Reshma Devi', B. Senthilkumaran ${ }^{3}$, R. Dada ${ }^{5}, K_{\text {. Singh }}{ }^{6}$, R. Singh ${ }^{7}$, N. J. Gupta ${ }^{8}$, B. N. Chakravarth ${ }^{8}$, M. Deendayal', K. V. Chary ${ }^{4}$, A. Anand ${ }^{2}$, Y. Sharma ${ }^{1}$, D. Gedala Rao ${ }^{10}$, K. C. Mantravadi ${ }^{10}$, J. Gopalakrishnan ${ }^{11}$, K. Thangaraj $^{1}$

${ }^{I}$ Centre for Cellular and Molecular Biology, Hyderabad, India, ${ }^{2}$ Jawaharlal Nehru Centre for Advanced Scientific Research (JNCASR), Bengaluru, India, ${ }^{3}$ University of Hyderabad, Hyderabad, India, ${ }^{4}$ Tata Institute of Fundamental Research (TIFR), Mumbai, India, ${ }^{5}$ All India Institute of Medical Sciences, New Delhi, India, ${ }^{6}$ Department of Molecular and Human Genetics, Banaras Hindu University (BHU), Varanasi, India, ${ }^{7}$ Division of Endocrinology, CSIRCentral Drug Research Institute, Lucknow, India, ${ }^{8}$ Institute of Reproductive Medicine, Kolkata, India, ${ }^{9}$ Infertility Institute and Research Centre, Secunderabad, India, ${ }^{10}$ Oasis Centre for Reproductive Medicine, Hyderabad, India, ${ }^{11}$ Center of Molecular Medicine, Cologne, Germany 
Introduction: Approximately, $10-15 \%$ of the couples are infertile worldwide and male factor alone accounts for $30-50 \%$ of infertility. The etiology in large proportion of infertile men still remains unknown. Therefore, to identify novel genetic factors we selected CETN1 based on our exome sequencing studies, sequenced it in large cohort of case-controls and performed various functional studies.

Materials and Methods: We performed exome sequencing of 44 idiopathic infertile men. CETN1 was one of the seven potential candidates identified ; hence, we further sequenced CETN1 in 1200 infertile men and 900 fertile men. Functional characterization of two variants from CETN1 was performed using various biophysical (CD, 2DHSQC etc.,), cell biology (using different cell lines and iPSC) approaches and luciferase assays. Quantitative estimation of Centrin-1 in oligospermic men with IVF failure history was done by ELISA.

Results: Two variants; rs61734344 (p.Met72Thr) and rs367716858 (5'UTR) showed significant association with infertility (p-values: $8.46 \mathrm{e}-06$ and 0.001 , respectively). Biophysical studies showed that mutation p.Met72Thr alters calcium-binding affinity and calcium-induced structural changes in Centrin-1. Somatic cells and differentiated iPSC over-expressing p.Met72Thr showed higher multipolar cells, fragmented nuclei and cell death. Studies using RPE cell lines showed that p.Met72Thr affects ciliary assembly dynamics and cell proliferation. Whereas, the 5'UTR mutation leads to over-expression of Centrin-1 due to loss of potential methylation site. Further, the reproductive outcome was markedly affected in patients with higher levels of Centrin-1 protein in their sperm centrosomes. This is the first study to demonstrate the role of CETN1 in human spermatogenesis and male fertility.

D.V.S. Sudhakar: None. S. Jaishankar: None. R. Phanindranath: None. A. Pawar: None. V. Jayaraman: None. D. Verma: None. J. Reshma Devi: None. B. Senthilkumaran: None. R. Dada: None. K. Singh: None. R. Singh: None. N.J. Gupta: None. B.N. Chakravarth: None. M. Deendayal: None. K.V. Chary: None. A. Anand: None. Y. Sharma: None. D. Gedala Rao: None. K.C. Mantravadi: None. J. Gopalakrishnan: None. K. Thangaraj: None.

\section{C04.6}

Duplication and deletion of key SOX9 enhancers cause sex reversal in humans

\section{B. M. Croft, T. Ohnesorg, A. H. Sinclair}

Murdoch Children's Research Institute, Melbourne, Australia
Introduction: Disorders of sex development (DSDs) are conditions affecting development of the gonads or genitalia. Variants in two key genes, $S R Y$ and its target $S O X 9$, are an established cause of 46,XY DSD, but the genetic basis of many DSDs remains unknown. SRY-mediated SOX9 upregulation in the early gonad is crucial for testis development, yet the regulatory elements underlying this have not been identified in humans.

Methods: We analysed copy number variations (CNVs) in the upstream regulatory region of SOX9 in DNA from patients with DSD, allowing us to define several minimal critical regions for sex-reversal.

Results: We redefined the upstream regulatory landscape of human SOX9. Using new patient data, we refined the $32.5 \mathrm{~kb}$ XYSR and $24 \mathrm{~kb}$ RevSex intervals and analysed these genomic regions using bioinformatic and luciferase tiling approaches, to identify three putative enhancers $5^{\prime}$ of SOX9. In cell-based reporter assays these enhancers responded to different combinations of testis-specific regulators including SRY, SF1 and SOX9 itself. When combined, all three enhancers show synergistic activity, significantly increasing their individual enhancer activity.

In vivo, deletion of these three enhancers in mice resulted in different outcomes ranging from: no apparent effect to reduced Sox 9 transcription and complete sex reversal.

Conclusion: This is the first study to identify SOX9 enhancers that, when duplicated or deleted, result in 46,XX or $46, \mathrm{XY}$ sex reversal, respectively. These enhancers provide a hitherto missing link by which SRY activates SOX9 initiation, upregulation and maintenance in humans, and establish SOX9 enhancer mutations as a significant cause of DSD.

B.M. Croft: None. T. Ohnesorg: None. A.H. Sinclair: None.

\section{C05}

Developmental disorders 1

\section{C05.1}

Human and mouse gene essentiality screens allow to identify candidate genes for developmental disorders

\section{Munoz Fuentes ${ }^{\text {I, P. } \text { Cacheiro }^{2} \text {, T. H. Meehan }}{ }^{\text {, }}$ D. Smedley ${ }^{2}$}

${ }^{1}$ EMBL-EBI, Cambridge, United Kingdom, ${ }^{2}$ Queen Mary University of London, London, United Kingdom

The identification of the causal variants of rare genetic diseases from voluminous patient sequencing data is an ongoing challenge. Here we combine cellular essentiality screen data with in vivo organismal gene essentiality from 
the International Mouse Phenotyping Consortium to aid in the prioritization of candidate disease genes. We categorized genes within a spectrum of phenotypic effects, and identified a Developmental Lethal (DL) category, which we define as genes not essential for cell survival but required for organismal development. With mouse viability data available for $\sim 4,900$ genes, 764 genes fall into the DL category. We found that this category presents a significant overrepresentation of Mendelian disease genes (OMIM and Orphanet associations, 2.5-fold increase odds), and an even higher enrichment (3.2-fold increase) for human genes associated with developmental disorders (from the Deciphering Developmental Disorders Study, DDD). We further investigated DL genes that were: (i) not described as associated with human disease by OMIM, Orphanet or DDD; and (ii) highly intolerant to LoF mutations, comprising 164 genes. Of these, 24 genes overlap with those containing heterozygous functional de novo variants in undiagnosed cases from the DDD study (DDD Research Variants). Further investigation of this set of genes is ongoing by comparing the clinical features of developmental disorders with homozygous and heterozygous knockout mouse models, to identify potential disease variants in cases with undiagnosed developmental disorders.

V. Munoz Fuentes: None. P. Cacheiro: None. T.H. Meehan: None. D. Smedley: None.

\section{C05.2}

C-type natriuretic peptide improves growth retardation in a mouse model of cardio-facio-cutaneous syndrome associated with a Braf mutation

\section{S. Inoue ${ }^{1}$, N. Morozumi ${ }^{2}$, K. Yoshikiyo ${ }^{2,3}$, H. Maeda ${ }^{2,4}$, Y. Aoki ${ }^{1}$}

${ }^{I}$ Department of Medical Genetics, Tohoku University School of Medicine, Sendai, Japan, ${ }^{2}$ Immunology \& Inflammatory Disease Field, Asubio Pharma Co., LTD, Kobe, Japan, ${ }^{3}$ Cell Therapy Research Laboratories, Daiichi Sankyo CO., LTD, Tokyo, Japan, ${ }^{4}$ End-Organ Disease Laboratories, Daiichi Sankyo CO., LTD, Tokyo, Japan

Cardio-facio-cutaneous (CFC) syndrome, a genetic disorder caused by germline mutations in BRAF, KRAS, MAP $2 K 1$ and $M A P 2 K 2$, is characterized by growth retardation, heart defects, dysmorphic facial appearance and dermatologic abnormalities. We have previously reported that knock-in mice $\left(\right.$ Braf $^{2241 R /+}$ ) expressing the CFC syndrome-associated mutation, Braf Q241R, showed growth retardation because of gastrointestinal dysfunction. However, other factors associated with growth retardation, including chondrogenesis and endocrinological profile, have not been examined.
Here, we show that 3- and 4-week-old Braf $f^{2241 R /+}$ mice have decreased body weight and length, as well as reduced growth plate width in the proximal tibiae. Furthermore, proliferative and hypertrophic chondrocyte zones of the growth plate were reduced in $B r a f^{2241 R /+}$ mice compared with control mice. Immunohistological analysis revealed that ERK activation was enhanced in hypertrophic chondrocytes in Braf ${ }^{Q 24 I R /+}$ mice. In accordance with growth retardation and reduced growth plate width, decreased serum levels of insulin-like growth factor 1 (IGF-1) and IGF binding protein 3 (IGFBP-3) were observed in Braf ${ }^{Q 241 R /+}$ mice at 3 and 4 weeks of age. In contrast, serum growth hormone levels were comparable in control and Braf $f^{Q 241 R /+}$ mice. Treatment with C-type natriuretic peptide (CNP), a stimulator of endochondral bone growth and a potent inhibitor of the FGFR3-RAF1-MEK/ERK signaling, increased body and tail lengths in control and Braf $f^{Q 241 R /+}$ mice. In conclusion, ERK activation in chondrocytes and low serum IGF-1/IGFBP-3 levels could be associated with the growth retardation observed in Braf ${ }^{241 R /+}$ mice. Our data also suggest that CNP is a potential therapeutic target in CFC syndrome and other RASopathies.

S. Inoue: None. N. Morozumi: None. K. Yoshikiyo: None. H. Maeda: None. Y. Aoki: None.

\section{C05.3}

Towards the treatment of Cantú syndrome

H. I. Roessler', C. McClenaghan ${ }^{2}$, Y. Huang ${ }^{2}$, C. M. Halabi ${ }^{2,3}$, T. Harter ${ }^{2}$, S. Savelberg ${ }^{1}$, F. Tessadori, ${ }^{4,1}$, J. Bakkers ${ }^{4,5}$, A. Kovacs ${ }^{6}$, M. S. Remedi ${ }^{2,7}$, E. P. Kirk ${ }^{8,9}$, I. Scurr ${ }^{10}$, S. F. Smithson ${ }^{10}$, M. M. van Haelst ${ }^{11,12}$, D. K. Grange ${ }^{2,3}$, C. G. Nichols ${ }^{2}$, G. van Haaften ${ }^{I}$

${ }^{1}$ Department of Genetics, Center for Molecular Medicine, University Medical Center Utrecht, Utrecht, Netherlands, ${ }^{2}$ Center for the Investigation of Membrane Excitability Diseases (CIMED), and Department of Cell Biology and Physiology, Washington University School of Medicine, St. Louis, MO, United States, ${ }^{3}$ Center for the Investigation of Membrane Excitability Diseases (CIMED), and Departments of Pediatrics, Washington University School of Medicine, St. Louis, MO, United States, ${ }^{4}$ Hubrecht Institute-KNAW and University Medical Centre Utrecht, Utrecht, Netherlands, ${ }^{5}$ Department of Medical Physiology, Division of Heart and Lungs, University Medical Center Utrecht, Utrecht, Netherlands, ${ }^{6}$ Center for the Investigation of Membrane Excitability Diseases (CIMED), Medicine, Washington University School of Medicine, St. Louis, St. Louis, MO, United States, ${ }^{7}$ Center for the Investigation of Membrane Excitability Diseases (CIMED), Medicine, Washington University School of Medicine, St. Louis, MO, United States, ${ }^{8}$ Dept of Medical Genetics, Sydney 
Children's Hospital, Randwick NSW, Australia, ${ }^{9}$ School of Women's and Children's Health, University of New South Wales, Randwick NSW, Australia, ${ }^{10}$ Department of Clinical Genetics, St Michael's Hospital, Bristol, United Kingdom, ${ }^{11}$ Department of Clinical Genetics, Amsterdam Medical Center, University of Amsterdam, Amsterdam, Netherlands, ${ }^{12}$ Department of Clinical Genetics, VU Medical Center, VU University Amsterdam, Amsterdam, Netherlands

Cantú Syndrome (CS) is a rare genetic disorder caused by gain-of-function (GOF) mutations in genes encoding the pore-forming (Kir6.1, KCNJ8) and regulatory (SUR2, $A B C C 9$ ) subunits of ATP-sensitive potassium (KATP) channels. CS patients present with hypertrichosis and facial anomalies alongside a varying range of cardiovascular abnormalities. To generate a critical mass patients to find further clinical characteristics of the disease as well as to ensure a rapid progression towards future interventional studies we developed a standardized registry. Clinical data is obtained during annual Cantú research clinics. CS is debilitating with no targeted therapy available. Clinical management currently involves symptomatic treatments to address second-order complications such as heart failure. The off-patent KATP inhibitor glibenclamide which is already widely applied in clinic to block GOF KATP channels involved in Type 2 diabetes holds promise as a potential treatment for CS. In order to perform therapeutic drug screening, we have developed novel CS animal models in which disease-causing mutations were knocked-in to the endogenous $A B C C 9$ loci in zebrafish and mice using CRISPR/Cas9 genome engineering. Both models recapitulate key cardiovascular features of CS which significantly reverse after glibenclamide administration. Hypercontractility and abnormally high-output were reversed in zebrafish larvae whilst cardiac hypertrophy in mice was similarly attenuated. No sustained hypoglycemia was observed after chronic exposure to glibenclamide in mice. These results provide key pre-clinical evidence for the in vivo efficacy of glibenclamide for the treatment of CS. Hence, we intend to perform a clinical trial to test glibenclamide in CS patients.

E-Rare 2014 grant 'CantuTreat' (I-2101-B26)

H.I. Roessler: None. C. McClenaghan: None. Y. Huang: None. C.M. Halabi: None. T. Harter: None. S. Savelberg: None. F. Tessadori: None. J. Bakkers: None. A. Kovacs: None. M.S. Remedi: None. E.P. Kirk: None. I. Scurr: None. S.F. Smithson: None. M.M. van Haelst: None. D.K. Grange: None. C.G. Nichols: None. G. van Haaften: None.

\section{C05.4}

A restricted spectrum of $K M T 2 D$ variants cause a multiple malformations disorder distinct from Kabuki syndrome
S. Cuvertino' ${ }^{1}$, V. Hartill ${ }^{2}$, A. Colyer ${ }^{3}$, F. Nadat ${ }^{3}$, N. Nair ${ }^{4}$, A. Stevens ${ }^{5}$, T. Garner ${ }^{5}$, V. Faundes ${ }^{1}$, S. Kimber ${ }^{6}$, F. Flinter ${ }^{7}$, S. A. Lynch $^{8}$, N. Canham ${ }^{9}$, The Deciphering Developmental Disorders Study, the 100,000 Genomes Project, B. Jackson ${ }^{3}$, G. M. Squeo ${ }^{10}$, S. Venuto ${ }^{10}$, G. Merla $^{10}$, E. Sheridan ${ }^{2}$, C. A. Johnson ${ }^{2}$, S. Banka ${ }^{11}$

${ }^{I}$ Division of Evolution and Genomic Sciences, School of Biological Sciences, Faculty of Biology, Medicine, and Health, The University of Manchester, Manchester, United Kingdom, ${ }^{2}$ Leeds Institute of Medical Research, Faculty of Medicine and Health. The University of Leeds, Leeds, United Kingdom, ${ }^{3}$ Faculty of Biological Sciences, The University of Leeds, Leeds, United Kingdom, ${ }^{4}$ Division of Musculoskeletal \& Dermatological Sciences, School of Biological Sciences, Faculty of Biology, Medicine, and Health, The University of Manchester, Manchester, United Kingdom, ${ }^{5}$ Division of Developmental Biology \& Medicine, School of Biological Sciences, Faculty of Biology, Medicine, and Health, The University of Manchester, Manchester, United Kingdom, ${ }^{6}$ Division of Cell Matrix Biology and Regenerative Medicine, School of Biological Sciences, Faculty of Biology, Medicine, and Health, The University of Manchester, Manchester, United Kingdom, ${ }^{7}$ Department of Clinical Genetics, Guy's \& St Thomas' NHS Foundation Trust, London, United Kingdom, ${ }^{8}$ School of Medicine, Children's University Hospital, Dublin, Ireland, ${ }^{9}$ Clinical Genetics, Liverpool Women's NHD Foundation Trust, Liverpool, United Kingdom, ${ }^{10}$ Division of Medical Genetics, Fondazione IRCCS Casa Sollievo della Sofferenza, San Giovanni Rotondo, Foggia, Italy, ${ }^{11}$ Manchester Centre for Genomic Medicine, St. Mary's Hospital, Manchester University Foundation NHS Trust, Health Innovation Manchester, Manchester, United Kingdom

Introduction: $K M T 2 D$ encodes a $\mathrm{H} 3 \mathrm{~K} 4$ methyltransferase made of 5537 amino acids. Loss-of-function KMT2D variants cause Kabuki syndrome (KS), characterised by intellectual disability, typical facial dysmorphism and multiple malformations of heart, kidneys and palate.

Results: Through targeted whole exome and genome sequencing we ascertained 10 individuals with de novo or inherited missense $K M T 2 D$ variants restricted to highly conserved residues within a central region predicted to form a coiled-coil dimer. All individuals presented with clinical features, strikingly distinct from KS, consisting of (a) varying combinations of branchial cysts, external ear malformations, ear pits, semi-circular canal anomalies, hearing loss, microphthalmia, lacrimal duct problems, choanal atresia, absent/hypoplastic nipples and hypothyroidism; (b) absence of typical facial features of KS; and (c) normal development in most individuals. KMT2D expression levels in fibroblast cells from two affected individuals were not 
altered compared to controls. However, mutant fibroblast had slightly decreased proliferation and migration compared to controls. The DNA methylation pattern in peripheral blood samples derived from affected individuals showed a specific signature distinct from that of KS. Circular dichroism performed on the purified KMT2D coiled-coil domain showed that alpha-helical secondary structure in the wild-type protein was perturbed by the missense variants.

Conclusion: We show that a restricted spectrum of pathogenic KMT2D missense variants results in a novel malformation disorder distinct from KS. This adds to the growing list of allelic disorders caused by mutations in chromatin remodelling genes and provides insights into function of previously unstudied region of this important protein.

S. Cuvertino: None. V. Hartill: None. A. Colyer: None. F. Nadat: None. N. Nair: None. A. Stevens: None. T. Garner: None. V. Faundes: None. S. Kimber: None. F. Flinter: None. S.A. Lynch: None. N. Canham: None. B. Jackson: None. G.M. Squeo: None. S. Venuto: None. G. Merla: None. E. Sheridan: None. C.A. Johnson: None. S. Banka: None.

\section{C05.5}

De novo C-terminal truncating mutations in $M N 1$ cause a neurodevelopmental syndrome with distinctive facial features

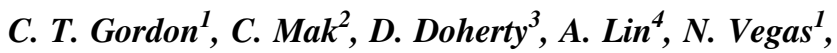
M. Cho ${ }^{5}$, G. Viot ${ }^{6}$, C. Dimartino ${ }^{1}$, J. Weisfeld-Adams ${ }^{7}$, D. Lessel ${ }^{8}$, S. Joss ${ }^{9}, C$. Li $^{10}$, C. Gonzaga-Jaureguili, Y. Zarate ${ }^{12}$, D. Horn ${ }^{13}$, C. Troyer ${ }^{14}$, S. Kant ${ }^{15}$, G. Leung ${ }^{2}$, A. Barone ${ }^{16}$, S. Yang ${ }^{5}$, E. Bend ${ }^{17}$, C. Roadhouse ${ }^{10}$, F. Zahir ${ }^{18}$, E. Stolerman ${ }^{17}$, T. Bienvenu ${ }^{19}$, N. Orenstein ${ }^{20}$, W. Dobyns ${ }^{3}$, J. Shieh ${ }^{21}$, D. Waggoner ${ }^{22}$, K. Gripp ${ }^{23}$, M. Parker $^{24}$, J. Stoler ${ }^{25}$, S. Lyonnet ${ }^{1}$, V. Cormier-Daire ${ }^{1}$, D. Viskochil ${ }^{26}$, T. Hoffman ${ }^{27}$, J. Amiel ${ }^{\text {I B. Bhung }}{ }^{2}$

${ }^{1}$ Institut Imagine, INSERM U1163, Paris, France, ${ }^{2}$ University of Hong Kong, Hong Kong, China, ${ }^{3}$ University of Washington, Seattle, WA, United States, ${ }^{4}$ MassGeneral Hospital for Children, Boston, MA, United States, ${ }^{5}$ GeneDx, Gaithersburg, MD, United States, ${ }^{6}$ Hôpital Cochin, Paris, France, ${ }^{7}$ University of Colorado, Aurora, CO, United States, ${ }^{8}$ University Medical Center HamburgEppendorf, Hamburg, Germany, ${ }^{9}$ West of Scotland Regional Genetics Service, Glasgow, United Kingdom, ${ }^{10}$ McMaster University Medical Center, Hamilton, ON, Canada, ${ }^{11}$ Regeneron Genetics Center, Tarrytown, NY, United States, ${ }^{12}$ University of Arkansas for Medical Sciences, Little Rock, AR, United States, ${ }^{13}$ Institut für Medizinische Genetik und Humangenetik, Berlin, Germany,

${ }^{14}$ University of Virginia, Charlottesville, VA, United States,
${ }^{15}$ Leiden University Medical Center, Leiden, Netherlands, ${ }^{16}$ Children's Hospital of Philadelphia, Philadelphia, PA, United States, ${ }^{17}$ Greenwood Genetic Center, Greenwood, SC, United States, ${ }^{18}$ University of British Columbia, Vancouver, BC, Canada, ${ }^{19}$ Institut Cochin, Paris, France, ${ }^{20}$ Tel Aviv University, Tel Aviv, Israel, ${ }^{21}$ University of California San Francisco, San Francisco, CA, United States, ${ }^{22}$ University of Chicago, Chicago, IL, United States, ${ }^{23}$ A. I. duPont Hospital for Children/Nemours, Wilmington, DE, United States, ${ }^{24}$ Sheffield Children's Hospital, Sheffield, United Kingdom, ${ }^{25}$ Boston Children's Hospital, Boston, MA, United States, ${ }^{26}$ University of Utah, Salt Lake City, UT, United States, ${ }^{27}$ Southern California Kaiser Permanente Medical Group, Anaheim, CA, United States

The meningioma (disrupted in balanced translocation) 1 $(M N 1)$ gene encodes a transcriptional co-regulator without homology to other proteins, previously implicated in acute myeloid leukemia and development of the palate in mice. We identified a cluster of de novo truncating mutations in $M N 1$ in a cohort of 21 individuals with strikingly similar dysmorphic facial features, especially midface hypoplasia, and intellectual disability with severe expressive language delay. MN1 is comprised of two exons. All mutations, including the recurrent variant p.Arg $1295^{*}$ observed in 8/19 probands, fall in the terminal exon or the extreme $3^{\prime}$ region of exon 1 , and are therefore predicted to result in escape from nonsense-mediated mRNA decay. This was confirmed in fibroblasts from three individuals. The C-terminal 20 amino acids of MN1, which are lacking in all patients, are very highly conserved amongst vertebrate species, suggesting an important role for the C-terminal tail in MN1 function. Previous studies have described large deletions of a region encompassing $M N I$ and other genes in individuals with variable neurodevelopmental anomalies and nonspecific facial features (dissimilar to the facial gestalt of the patients reported here). We propose that the condition described here for the first time, MEningioma $1 \mathrm{C}$-terminal Truncation (MECT) syndrome, is not due to MNl haploinsufficiency but rather is the result of dominantly acting C-terminally truncated MN1 protein. In summary, our data show that MN1 plays a critical role in human craniofacial and brain development.

C.T. Gordon: None. C. Mak: None. D. Doherty: None. A. Lin: None. N. Vegas: None. M. Cho: None. G. Viot: None. C. Dimartino: None. J. Weisfeld-Adams: None. D. Lessel: None. S. Joss: None. C. Li: None. C. GonzagaJauregui: None. Y. Zarate: None. D. Horn: None. C. Troyer: None. S. Kant: None. G. Leung: None. A. Barone: None. S. Yang: None. E. Bend: None. C. Roadhouse: None. F. Zahir: None. E. Stolerman: None. T. Bienvenu: None. N. Orenstein: None. W. Dobyns: None. J. Shieh: None. D. Waggoner: None. K. Gripp: None. M. 
Parker: None. J. Stoler: None. S. Lyonnet: None. V. Cormier-Daire: None. D. Viskochil: None. T. Hoffman: None. J. Amiel: None. B. Chung: None.

\section{C05.6}

De novo missense mutations in the $\mathrm{X}$-linked TFE3 gene cause intellectual disability with pigmentary mosaicism and storage disorder-like features

D. Lehalle ${ }^{1,2,3}$, P. Vabres ${ }^{1}$, T. Bierhals ${ }^{4}$, M. T. Cho ${ }^{5}$, B. Cogne $^{6,7}$, M. Avila $^{1}$, V. Carmignac ${ }^{1}$, L. Duplomb-Jego ${ }^{1}$, E. De Bont ${ }^{8}$, Y. Duffourd ${ }^{1}$, F. Duijkers ${ }^{9}$, O. Elpeleg ${ }^{10}$, A. Fattal-Valevskil ${ }^{11}$, D. Genevieve ${ }^{12}$, A. Guimier ${ }^{13}$,

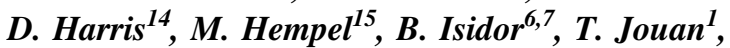
P. Kuentz ${ }^{1}$, K. Lichtenbelt ${ }^{16}$, V. Loik Ramey ${ }^{14}$, L. Pasquier ${ }^{17}$, J. St-Onge ${ }^{18,1}$, A. Sorlin ${ }^{1,2}$, J. Thevenon ${ }^{1,19}$, E. Torti $^{20}$, K. Van Gassen ${ }^{16}$, M. Van Haelst ${ }^{9}$, S. van Koningsbruggen $^{9}$, J. Riviere ${ }^{1,3,18}$, C. Thauvin ${ }^{1,2}$, J. Betschinger ${ }^{21}$, L. Faivre ${ }^{1,2}$

${ }^{1}$ Equipe GAD, INSERM LNC UMR 1231, Faculté de Médecine, Université de Bourgogne Franche-Comté, Dijon, France, ${ }^{2}$ Fédération Hospitalo-Universitaire Médecine Translationnelle et Anomalies du Développement (TRANS$L A D)$, Centre Hospitalier Universitaire Dijon et Université de Bourgogne, Dijon, France, ${ }^{3}$ Department of Human Genetics, Faculty of Medicine, McGill University, Montreal, QC, Canada, ${ }^{4}$ Institute of Human Genetics, University Medical Center Hamburg-Eppendorf, Martinistraße 52, 20246, Hamburg, Germany, ${ }^{5}$ GeneDx, Gaithersburg, $M D$, Bethesda, MD, United States, ${ }^{6} \mathrm{CHU}$ de Nantes, Service de Génétique Médicale, Nantes, France, ${ }^{7}$ L'institut du thorax, INSERM, CNRS, UNIV Nantes, CHU de Nantes, Nantes, France, ${ }^{8}$ Department of Pediatric Oncology/ Hematology, Beatrix Children's Hospital, University Medical Centre Groningen, Groningen, Netherlands, ${ }^{9}$ Department of Genetics, AUMC, Amsterdam, Netherlands, ${ }^{10}$ Monique and Jacques Roboh Department of Genetic Research, Hadassah-Hebrew University Medical Center, Jerusalem, Israel, ${ }^{11}$ Pediatric Neurology Institute, Tel Aviv Sourasky Medical Center, Sackler Faculty of Medicine, Tel Aviv University, Tel Aviv, Israel, ${ }^{12}$ Department of Clinical Genetics, Montpellier, France, ${ }^{13}$ Service de Génétique, Hôpital Necker-Enfants Malades, AP-HP, Paris, France, ${ }^{14}$ Division of Genetics and Genomics, Boston Children's Hospital, Boston, MA, United States, ${ }^{15}$ Institute of Human Genetics, University Medical Center Hamburg-Eppendorf, Martinistraße 52, 20246, Hamburg, Germany, ${ }^{16}$ Department of Genetics, University Medical Center Utrecht (UMCU), Utrecht, Netherlands, ${ }^{17}$ Service de génétique médicale, CHU Rennes, Rennes, France, ${ }^{18}$ Child Health and Human Development Program, Research Institute of the McGill University Health Centre, Montreal, QC,
Canada, ${ }^{19}$ Centre de Génétique, Hôpital Couple-Enfant, CHU de Grenoble-Alpes, La Tronche, Grenoble, France, ${ }^{20}$ GeneDx, Gaithersburg, MD, United States, ${ }^{21}$ Friedrich Miescher Institute for Biomedical Research, Basel, Switzerland

Introduction: Pigmentary mosaicism (PM) manifests by hypopigmentation along Blaschko's lines, often associated with intellectual disability. Molecular etiologies are still poorly elucidated. Together with new insights on the role for lysosomal signaling in embryonic stem cell differentiation, mutations in the X-linked transcription factor TFE3 have recently been reported in five patients with PM. Functional analysis suggested these mutations to result in ectopic nuclear gain-of-function Tfe 3 alleles.

Material and Methods: Subsequent datasharing allowed the clustering of de novo TFE3 variants identified by exome sequencing or TFE3 targeted sequencing on DNA extracted from leucocytes in patients referred for syndromic intellectual disability with or without PM.

Results: We describe the clinical and molecular data of the five previously published patients and six additional individuals harboring a de novo missense TFE3 mutation. The seven females and four males presented with pigmentation anomalies on Blaschko's lines, severe intellectual disability, epilepsy, metabolic anomalies (hepatomegaly, obesity, hypoglycemia), growth retardation and recognizable facial dysmorphism with progressive coarsening. The mutation was at a mosaic state in two male patients. Two of the eight identified mutations were localized in exon 3 , six in exon 4; three were recurrent mutations.

Conclusion: This series of 11 individuals further delineates the specific storage disorder-like phenotype with pigmentary mosaicism ascribed to de novo TFE3 missense mutation in exons 3 and 4 . It confirms the identification of a novel $\mathrm{X}$-linked condition associated with mosaicism and dysregulation within the mTOR pathway, as well as a link between lysosomal signaling and human development.

D. Lehalle: None. P. Vabres: None. T. Bierhals: None. M.T. Cho: A. Employment (full or part-time); Significant; GeneDx. B. Cogne: None. M. Avila: None. V. Carmignac: None. L. Duplomb-Jego: None. E. De Bont: None. Y. Duffourd: None. F. Duijkers: None. O. Elpeleg: None. A. Fattal-Valevski: None. D. Genevieve: None. A. Guimier: None. D. Harris: None. M. Hempel: None. B. Isidor: None. T. Jouan: None. P. Kuentz: None. K. Lichtenbelt: None. V. Loik Ramey: None. L. Pasquier: None. J. St-Onge: None. A. Sorlin: None. J. Thevenon: None. E. Torti: A. Employment (full or part-time); Significant; GeneDx. K. Van Gassen: None. M. Van Haelst: None. S. van Koningsbruggen: None. J. Riviere: None. C. Thauvin: None. J. Betschinger: None. L. Faivre: None. 


\section{C06 Cellular dysfunctions}

\section{C06.1}

DTYMK deficiency is the cause of a novel vanishing brain disease

\section{J. M. Vanoevelen ${ }^{1}$, J. Bierau ${ }^{1}$, E. Kamsteeg ${ }^{2}$,} R. A. Wevers ${ }^{3}$, V. A. Bok ${ }^{4}$, M. van der Knaap ${ }^{5}$, M. Bugiani ${ }^{6}$, M. O' Driscoll $^{7}$, A. van den Wijngaard ${ }^{1}$, H. G. Brunner ${ }^{\text {I, L. Wang }}{ }^{8}$, C. T. R. M. Stumpel ${ }^{I}$

${ }^{1}$ Department of Clinical Genetics, Maastricht University Medical Centre+, Maastricht, Netherlands, ${ }^{2}$ Department of Human Genetics, Radboud UMC, Nijmegen, Netherlands, ${ }^{3}$ Translational Metabolic Laboratory, Radboud UMC, Nijmegen, Netherlands, ${ }^{4}$ Department of Pediatrics, Màxima Medical Center, Veldhoven, Netherlands, ${ }^{5}$ Department of Child Neurology, VU UMC, Amsterdam, Netherlands, ${ }^{6}$ Department of Neuropathology, VU UMC, Amsterdam, Netherlands, ${ }^{7}$ Genome Damage and Stability Centre, University of Sussex, Brighton, United Kingdom, ${ }^{8}$ Department of Anatomy, Physiology and Biochemistry, Swedish University of Agricultural Sciences, Uppsala, Sweden

Nucleotide metabolism is a complex process regulating processes including proliferation, DNA synthesis and repair. Here, we describe DTYMK deficiency as the cause of a novel vanishing brain disease in two unrelated patients. DTYMK encodes TMPK (thymidylate monophosphate kinase), a key enzyme in dTTP biosynthesis. The clinical phenotype is characterized by microcephaly, no growth in length and severe developmental delay. MRI revealed extreme loss of white matter in the cerebral cortex and absence of basal ganglia. Two compound heterozygous variants in DTYMK were detected in the first patient whereas a second patient carried a (third) homozygous variant in DTYMK. TMPK activity was undetectable in patient fibroblasts and a proliferation defect that was absent in control cells was observed. Homozygous dtymk mutant zebrafish show microcephaly, developmental delay and massive brain edema. TMPK activity in mutant zebrafish confirmed that the allele represents a loss-of-function. Furthermore, a striking reduction of cell proliferation was detected in the brain of mutant zebrafish larvae, as well as an impairment of the DNA-damage repair mechanism. These similarities between the human and zebrafish phenotype strongly suggest a causal link between TMPK deficiency and neurodegeneration. Current research is focused on the identification of the compensatory mechanism and the pathogenic mechanism underlying the vanishing white matter but reduced proliferation and a deficient DNA repair mechanism are suspected to play major roles. In summary, by combining genetic and biochemical approaches in a cellular and a zebrafish model we identified loss-of-function in DTYMK as the cause of a novel vanishing brain disease.

J.M. Vanoevelen: None. J. Bierau: None. E. Kamsteeg: None. R.A. Wevers: None. V.A. Bok: None. M. van der Knaap: None. M. Bugiani: None. M. O' Driscoll: None. A. van den Wijngaard: None. H.G. Brunner: None. L. Wang: None. C.T.R.M. Stumpel: None.

\section{C06.2}

\section{DEGS1 Mutation causes sphingolipidosis}

\section{A. Dolgin 1 , R. Straussberg ${ }^{2,3}$, R. Xu ${ }^{4}$, I. Mileva, Y. Yogev ${ }^{1}$, C. Mao ${ }^{4}$, O. S. Birk ${ }^{5,1}$}

${ }^{I}$ The Morris Kahn Laboratory of Human Genetics, National Institute for Biotechnology in the Negev and Faculty of Health Sciences, Ben-Gurion University of the Negev, Beer Sheva, Israel, ${ }^{2}$ Neurogenetics Clinic, Neurology Unit, Schneider Children Medical Center, Petah Tikvah, Israel, ${ }^{3}$ Sackler Faculty of Medicine, Tel Aviv University, Tel Aviv, Israel, ${ }^{4}$ Department of Medicine and Stony Brook Cancer Center, The State University of New York at Stony Brook, New York, NY, United States, ${ }^{5}$ Genetics Institute, Soroka University Medical Center, Beer Sheva, Israel

Introduction: Mutations in enzymes of lipid synthesis and metabolism have been demonstrated to cause sphingolipidoses.

Materials and Methods: linkage analysis, whole exome sequencing, lipidomics analysis.

Results: We describe an autosomal recessive neurological disorder affecting consanguineous kindred. All four affected individuals, born at term following normal pregnancies, had mild to severe intellectual disability, spastic quadriplegia, scoliosis and epilepsy in most, with no dysmorphic features. Brain MRI demonstrated leukodystrophy with abnormal hyperintense signal in the periventricular perioccipital region and thinning of corpus callosum. Notably, affected individuals were asymptomatic at early infancy and developed normally until the age of 8-18 months, when deterioration ensued. Homozygosity mapping identified a single $8.7 \mathrm{Mb}$ disease-associated locus on chromosome 1q41-1q42.13 between rs1511695 and rs537250 (two-point LOD score 2.1). Whole exome sequencing identified within this locus a single diseaseassociated homozygous mutation in DEGS1, encoding C4dihydroceramide desaturase, an enzyme of the ceramide synthesis pathway. The missense mutation, segregating within the family as expected for recessive heredity, affects an evolutionary-conserved amino acid of both isoforms of $D E G S 1$ and was not found in a homozygous state in ExAC and gnomAD databases or in 300 ethnically matched 
individuals. Analysis of whole blood of affected individuals demonstrated augmented levels of dihydroceramides, dihydrosphingosine, dihydrosphingosine-1-phosphate and dihydrosphingomyelins with reduced levels of ceramide, sphingosine, sphingosine-1-phosphate and monohexosylceramides, as expected in malfunction of $\mathrm{C} 4$ dihydroceramide desaturase.

Conclusion: We demonstrate that a mutation in DEGS1 causes a sphingolipidosis presenting at age 8-18 months with severe leukodystrophy, implying future treatment modalities for this severe neurologic disease.

V.A. Dolgin: None. R. Straussberg: None. R. Xu: None. I. Mileva: None. Y. Yogev: None. C. Mao: None. O.S. Birk: None.

\section{C06.3}

MAGT1-CDG vs. XMEN: two faces of a novel glycosylation disorder

E. Blommaert', R. Péanne ${ }^{1}$, N. A. Cherepanova ${ }^{2}$, D. Rymen ${ }^{3}$, J. Jaeken ${ }^{4}$, R. Schrijvers ${ }^{5}$, F. Foulquier ${ }^{6}$, R. Gilmore ${ }^{2}$, G. Matthijs ${ }^{1}$

${ }^{1}$ Department of Human Genetics, KU Leuven, Leuven, Belgium, ${ }^{2}$ Department of Biochemistry and Molecular Pharmacology, University of Massachusetts Medical School, Worcester, MA, United States, ${ }^{3}$ Division of Metabolic Diseases, University Children's Hospital, Zurich, Switzerland, ${ }^{4}$ Center for Metabolic Diseases, $K U$ Leuven, Leuven, Belgium, ${ }^{5}$ Department of microbiology and clinical immunology, KU Leuven, Leuven, Belgium, ${ }^{6}$ Unité de Glycobiologie Structurale et Fonctionnelle, Univ. Lille, Lille, France

Introduction: Congenital Disorders of Glycosylation (CDG) are a group of rare, genetic diseases. Here, we identified a novel CDG caused by hemizygous mutations in the X-linked MAGT1. Patient 1 and 2, both boys, present with intellectual and developmental disability. Furthermore, a third MAGT1-deficient patient (P3) with an immunodeficiency characterised by Epstein-Barr virus (EBV) infections was identified. MAGT1 is a subunit of the oligosaccharyltransferase (OST) complex, with an important role in N-glycosylation. However, mutations in MAGT1 have been described to cause an immunodeficiency (XMEN), characterised by EBV infections. These studies claim that MAGT1 is a $\mathrm{Mg}^{2+}$ transporter.

Methods: Protein expression levels of OST subunits were evaluated in patient and CRISPR K.O. cell lines. Radiolabelling of glycoproteins was performed to detect $\mathrm{N}$ glycosylation defects.

Results: Protein levels of MAGT1 were unaffected for P1 (missense mutation) and absent for P2 and P3 (nonsense mutations). Expression of TUSC3, the homologue protein of MAGT1, was increased in all patient-derived fibroblasts. By radiolabelling we observed that the patient fibroblasts have a mild glycosylation defect. This is in line with reports showing that enhanced expression of TUSC3 mitigates the MAGT1 defect. Interestingly, glycoproteins were strongly hypoglycosylated in P3 lymphocytes, as they don't express TUSC3. Lastly, complementation experiments with mutated MAGT1 did not rescue the defective glycosylation in MAGT1 $^{-/-}$TUSC $^{-/-}$HEK293 cells, indicating that all three mutations are pathogenic.

Conclusion: We showed that glycosylation is impaired in all patients. Hence, we delineate MAGT1-CDG as a novel glycosylation disorder associated with two different clinical phenotypes.

Funding: ERA-NET $\mathrm{N}^{\circ} 64578, \mathrm{NIH} \mathrm{N}^{\circ} \mathrm{GM} 43768$

E. Blommaert: None. R. Péanne: None. N.A. Cherepanova: None. D. Rymen: None. J. Jaeken: None. R. Schrijvers: None. F. Foulquier: None. R. Gilmore: None. G. Matthijs: None.

\section{C06.4}

Implication of LRPG variants in familial hypercholesterolemia
Y. Ghaleb ${ }^{1,2}$, S. ElBitar ${ }^{1,2}$, A. Loste ${ }^{1}$, P. El khoury ${ }^{1,2}$, Y. Abou-khalil ${ }^{1,2}$, G. Nicolas ${ }^{3}$, M. Le Borgne ${ }^{1}$, P. Moulin 4 , M. Di-filippo ${ }^{5}$, S. Charriere $^{4}$, H. Iliozer $^{4}$, J. Rabès $^{6,7}$, C. Boileau ${ }^{1,8,9}$, M. Abifadel ${ }^{1,2}$, M. Varret $^{1}$

${ }^{1}$ LVTS INSERM U1148, Paris, France, ${ }^{2}$ Faculty of Pharmacy, Saint-Joseph University, Beirut, Lebanon, ${ }^{3}$ INSERM UMR 1149, Paris, France, ${ }^{4}$ Hospices Civils de Lyon, Louis Pradel Cardiovascular Hospital, Department of Endocrinology, nutrition and metabolic diseases, Lyon, France, ${ }^{5}$ Hospices Civils de Lyon, Department of Biochemistry and Molecular Biology, Lyon, France, ${ }^{6} A P-H P$, HUPIFO, Ambroise Paré Hospital, Laboratory of Biochemistry and Molecular Genetics, Paris, France, ${ }^{7}$ UVS, UFR des Sciences de la Santé Simone Veil, Montigny-LeBretonneux, France, ${ }^{8}$ Paris Diderot University, Paris, France, ${ }^{9}$ AP-HP, CHU Xavier Bichat, Genetic Department, Paris, France

Introduction: Familial hypercholesterolemia (FH) is a genetic disorder characterized by elevated plasma LDL-C levels. It is caused by mutations in five genes: $L D L R$, APOB, PCSK9, APOE, LDLRAP1. Other FH-causing genes remain to be characterized. The aim of this study conducted in a large French family and in unrelated French hypercholesterolemic probands is to identify new FH-causing genes which can explain the mutation negative cases. 
Materials: The studied FH family (20 members including 6 affected over 3 generations) and probands were recruited through the French FH Research Network and mutations in $L D L R, A P O B, P C S K 9$, APOE were excluded. A whole exome sequencing (WES) was realized in 3 affected members of the family and Sanger sequencing in the 84 probands. Analysis of variant effects was assessed in silico. Then we investigated the activity of the candidate gene and its variants in vitro. Wildtype and mutant plasmids were constructed and transfected into HEK293T cells. We determined protein expression and LDL uptake by FACS analysis.

Results: WES identified a heterozygous variant segregating in the family in LRP6 (p.(Val1382Phe)). Four other rare and disease causing variants in this gene were found in 4 unrelated probands (p.(Tyr972Cys), p.(Thr1479Ile), p. (Tyr1584Asn), p.(Ser1612Phe)). Functional studies showed that two variants decrease significantly the expression of LRP6 at the cell surface and increase slightly but not significantly LDL uptake. However, the expression of LDL receptor was not affected by any variant.

Conclusion: This study brings new data concerning the role of LRP6 in LDL uptake. However, several points remain to be elucidated.

Y. Ghaleb: None. S. EIBitar: None. A. Loste: None. P. El khoury: None. Y. Abou-khalil: None. G. Nicolas: None. M. Le Borgne: None. P. Moulin: None. M. Difilippo: None. S. Charriere: None. H. Iliozer: None. J. Rabès: None. C. Boileau: None. M. Abifadel: None. M. Varret: None.

\section{C06.5}

Deciphering altered inhibitor G-protein signaling in the cardiac dysfunction underlying Intellectual Developmental Disorder with Cardiac Arrhythmia (IDDCA) syndrome

\section{P. De Nittis ${ }^{1}$, A. Sarre ${ }^{2}$, J. Chrast ${ }^{1}$, W. F. Simonds ${ }^{3}$, T. Pedrazzini ${ }^{4}$, A. Reymond ${ }^{1}$}

${ }^{I}$ Center for Integrative Genomics, Lausanne, Switzerland, ${ }^{2}$ Cardiovascular Assessment Facility, University of Lausanne, Lausanne, Switzerland, ${ }^{3}$ Metabolic Diseases Branch/NIDDK, National Institutes of Health, Bethesda, $M D$, United States, ${ }^{4}$ Experimental Cardiology Unit, Department of Cardiovascular Medicine, University of Lausanne, Lausanne, Switzerland

Inactivating mutations in the guanine nucleotide-binding protein subunit- $\beta-5$ (GNB5) gene cause IDDCA syndrome, an autosomal recessive neurodevelopmental disorder associated with cognitive disability and cardiac arrhythmia. Knock-out of Gnb5 in mice results in structural and functional abnormalities of the brain. However, little is known about the role of Gnb5 in heart sinus conduction. Notably, all IDDCA individuals present heart bradycardia. Here we show that Gnb5-inhibitory signaling is essential for parasympathetic control of heart rate. We monitored i) heart electrophysiology in homozygous $(\mathrm{KO}, \mathrm{n}=8)$, heterozygous (HET, $\mathrm{n}=8$ ) and wild-type (WT, $\mathrm{n}=7$ ) mice using in vivo ECG telemetry, and ii) cardiac function by echocardiography. KO mice were smaller than WT and HET mice, and had a smaller heart, but exhibited better cardiac function, as judged by increased fractional shortening and ejection fraction. Whereas baseline ECG was not different between groups, $\mathrm{KO}$ mice treated with carbachol $(\mathrm{CCh})$, a parasympathomimetic, presented significant $\mathrm{CCh}$ induced bradycardia. In contrast, the anti-parasympathetic drug atropine had the same effect in KO, HET and WT mice. KO and WT showed similar responses to atenolol and isoprotenerol, respectively, agonist and antagonist of the $\beta$-adrenergic activity, suggesting that sympathetic modulation of the cardiac stimulation is not altered. Altogether, our data demonstrate that loss of negative regulation on the inhibitory G-protein signaling causes heart rate perturbations in Gnb5-KO mice. This effect is mainly driven by impaired parasympathetic activity. We anticipate that unravelling the mechanistic of Gnb5-signaling in the autonomic control of the heart will pave the way for future drug screening.

P. De Nittis: None. A. Sarre: None. J. Chrast: None. W.F. Simonds: None. T. Pedrazzini: None. A. Reymond: None.

C06.6

PCYT2 Variants Disrupt Etherlipid Biosynthesis and Cause a Complex Hereditary Spastic Paraplegia

J. H. McDermott ${ }^{1}$, F. Vaz ${ }^{2}$, M. Alders ${ }^{3}$, S. B. Wortmann ${ }^{4}$, S. Kölker ${ }^{5}$, M. L. Pras-Raves ${ }^{2}$, M. A. T. Vervaart ${ }^{2}$, H. Van Lenthe $^{2}$, A. C. M. Luyf $f^{2}$, H. L. Elfrink ${ }^{2}, K$. Metcalfe $^{I}$,

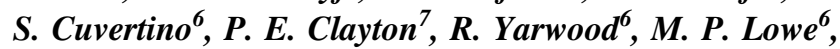
S. Lovell', R. C. Rogers ${ }^{8}$, A. H. C. Van Kampen', Deciphering Developmental Disorders Study, J. P. N. Ruiter $^{2}$, S. Ferdinandusse ${ }^{2}$, M. Van Weeghel', M. Engelen ${ }^{10}$, S. Banka ${ }^{1}$

${ }^{1}$ Manchester Centre for Genomic Medicine, Manchester, United Kingdom, ${ }^{2}$ Laboratory Genetic Metabolic Diseases, Amsterdam, Netherlands, ${ }^{3}$ Laboratory Genome Diagnostics, Amsterdam, Netherlands, ${ }^{4}$ Institute of Human Genetics, Munich, Germany, ${ }^{5}$ Division of Pediatric Neurology and Metabolic Medicine, Heidelberg, Germany, ${ }^{6}$ University of Manchester, Manchester, United Kingdom, ${ }^{7}$ Department of Pediatric Endocrinology, Manchester, United Kingdom, ${ }^{8}$ Greenwood Genetic Center, Greenwood, SC, United 
States, ${ }^{9}$ University of Amsterdam, Amsterdam, Netherlands,

${ }^{10}$ Department of Pediatric Neurology, Amsterdam, Netherlands

Introduction: PCYT2 encodes CTP:phosphoethanolamine cytidylyltransferase (ET), the rate-limiting enzyme for phosphatidylethanolamine (PE) synthesis via the CDPethanolamine pathway. $\mathrm{PE}$ is the most abundant membrane lipid that is enriched in the CNS.

Results: We identified five individuals from four families with biallelic PCYT2 variants via exome sequencing. One patient had compound heterozygous missense variants while all others possessed an identical homozygous lastexon nonsense variant. All patients had mild to severe global developmental delay, progressive spastic tetraparesis, seizures, regression and cerebral and cerebellar atrophy. Nystagmus and poor visual acuity were reported in four individuals. In patient fibroblasts, mRNA levels were normal whilst ET protein levels and enzyme activity were significantly decreased (but not absent). In silico analysis of missense variants showed that the substitutions probably affected binding, catalysis and protein stability. Hypomorphic G0 PCYT2 zebrafish generated using the CRISPRCas9 system had abnormal tail-fin morphology, whereas zebrafish with complete knockout of the gene were incompatible with life. Lipidomic analysis of patient fibroblasts demonstrated profound abnormalities in neutral etherlipid and etherphospholipids and revealed that in ET deficiency, the phosphatidyl-serine decarboxylase route is used to synthesize plasmalogens. Patient plasma lipidomics showed significant accumulation of phosphatidylcholine[O].

Conclusion: Hypomorphic biallelic PCYT2 variants cause a novel syndromic progressive neurological disorder and the complete loss of ET function is likely incompatible with life. Lipidomic abnormalities in patient tissues could be used as a biomarker facilitating diagnosis and interpretation of variants of uncertain significance. Finally, this study highlights the importance of etherlipid homeostasis in the development and function of the CNS.

J.H. McDermott: None. F. Vaz: None. M. Alders: None. S.B. Wortmann: None. S. Kölker: None. M.L. Pras-Raves: None. M.A.T. Vervaart: None. H. Van Lenthe: None. A.C.M. Luyf: None. H.L. Elfrink: None. K. Metcalfe: None. S. Cuvertino: None. P.E. Clayton: None. R. Yarwood: None. M.P. Lowe: None. S. Lovell: None. R.C. Rogers: None. A.H.C. Van Kampen: None. J. P.N. Ruiter: None. S. Ferdinandusse: None. M. Van Weeghel: None. M. Engelen: None. S. Banka: None.

\section{C07 Gene editing and reproduction}

\section{C07.1}

Experts' opinions on genome editing in humans: a collective construction of a disruptive technology

\section{Romano, H. C. Howard}

Uppsala University, Uppsala, Sweden

Germ line genome editing (GLGE) is a highly controversial matter, tending to divide the expert community revolving around it. To date, very few studies have explored in-depth the views of expert stakeholders. To help fill this gap, we interviewed 20 expert stakeholders in genome editing (academic laboratory and clinical researchers, bioethicists, lawyers, patient advocates) from Europe, North America, China and Japan. In our semi-guided interviews, we posed, among others, questions addressing legitimacy, roles and responsibilities, engagement of the public about different uses, and future scenarios in so doing constructing a collective idea of what GLGE is and could be. About legitimate uses, we found that many stakeholders are skeptical about the need to use GLGE because of ethical implications, environmental risks and the existence of more safer alternatives. Roles and responsibilities are called to be horizontal and diffused as much as possible to everybody (through adequate representation). The participation of the wider public is considered essential for transgenerational ethical dilemmas. Future scenarios are depicted as negative for, above all, women and women's bodies, the meaning and actual impact of parenting, and the creation of wider inequalities between social classes. In general, we understood that certain views are common among experts, such as the extreme caution which should guide the path from the lab to clinical trials. However, there are also many differences in perceived priorities, based on the anticipation of different cost-benefit balances and on the diverse predictions of GLGE's development and its potential impact on human kind.

V. Romano: None. H.C. Howard: None.

\section{C07.2}

How will new reproductive genetic technologies change genetically at-risk couples' reproductive decision making? Views on NIPD and gene modification

\section{I. van Dijke ${ }^{1}$, P. Lakeman ${ }^{2}$, I. B. Mathijssen ${ }^{2}$, M. C. Cornel ${ }^{3}$, L. Henneman ${ }^{3}$}

${ }^{1}$ Amsterdam UMC, Amsterdam Reproduction and Development Research Institute, Amsterdam, Netherlands, ${ }^{2}$ Amsterdam UMC, University of Amsterdam, Dept of Clinical Genetics, Amsterdam Reproduction and Development Research Institute (AR\&D), Amsterdam, Netherlands, 
${ }^{3}$ Amsterdam UMC, Vrije Universiteit Amsterdam, Dept of Clinical Genetics, Amsterdam Reproduction and Development Research Institute (AR\&D), Amsterdam, Netherlands

Genetically at-risk couples who want to avoid having an affected child have several reproductive options including prenatal diagnosis (PND) and preimplantation genetic diagnosis (PGD). In the future, non-invasive prenatal diagnosis (NIPD), germline gene modification (GGM) and somatic gene modification (SGM) might become available. This study explores if, and how, availability of possible future reproductive options would influence couples' decision making.

Interviews $(\mathrm{n}=25)$ were conducted with couples with a known increased risk of having a child with a genetic condition who received genetic counselling at one Dutch Clinical Genetics Centre between 2013-2018.

For couples, factors currently influencing decisionmaking for or against PND or PGD (e.g. attitude towards pregnancy termination, the impact of procedure) also played a role when discussing the new reproductive technologies. Couples were generally positive about NIPD as this would be more safe and enables earlier testing compared to PND. However, some who were against pregnancy termination would still prefer PGD. The perceived burden of PGD, embryo disposal, and increasing opportunities of having a 'healthy' embryo were considerations in favour of GGM. However, some considered GGM as unsafe, feared misuse and 'slippery slope' scenarios. SGM was generally not considered an option to anticipate on, since the affected child would still be born and subjected to treatment.

Genetically at-risk couples have mixed feelings: NIPD and GGM were more positively evaluated than SGM. The results suggest that shifts in use towards new technologies can be expected. Users' perspectives should be addressed for responsible implementation. Grant: Amsterdam Reproduction and Development Research Institute

I. van Dijke: None. P. Lakeman: None. I.B. Mathijssen: None. M.C. Cornel: None. L. Henneman: None.

\section{C07.3}

Perspectives of a Genetic Disease Community and Genetic Professionals on Germline Gene Editing

B. M. Hollister ${ }^{1}$, M. Gatter ${ }^{1}$, K. E. Abdallah ${ }^{1}$, A. Armsby ${ }^{2}$, A. J. Buscetta ${ }^{I}$, Y. Byeon ${ }^{I}$, K. E. Cooper ${ }^{I}$, S. Desine ${ }^{I}$, M. J. Hahn', K. E. Ormond ${ }^{3}$, V. L. Bonham ${ }^{I}$

${ }^{I}$ National Human Genome Research Institute, Bethesda, MD, United States, ${ }^{2}$ Oxford University Hospitals NHS Foundation Trust, Oxford, United Kingdom, ${ }^{3}$ Department of Genetics and Stanford Center for Biomedical Ethics at Stanford University School of Medicine, Stanford, CA, United States
Title: Perspectives of a Genetic Disease Community and Genetic Professionals on Germline Gene Editing

Introduction: Advances in CRISPR technology and the announcement of the first gene edited babies have sparked a global conversation about the future of human germline gene editing (GLGE). ESHG recommended that basic and pre-clinical germline research could be justified while acknowledging that therapeutic or preventive GLGE is not warranted at this time and merits more debate.

Internationally, policymakers, scientists and media have called for a global conversation including the voices of the public and relevant stakeholders. Our study contributes to this need by investigating the GLGE views of the sickle cell disease (SCD) community and genetic professionals.

Methods: In 2017, we utilized a mixed-methods approach, involving an original educational video on CRISPR genome editing, surveys, and 15 focus groups of United States-based SCD community members: six patient, six parent, and three physician groups. The findings were compared to an international survey of genetic professionals conducted from 2016-2017.

Results: Compared to genetics professionals, where $48.2 \%$ found GLGE to be morally acceptable, fewer in the SCD community supported GLGE $(24.0 \%, \mathrm{p}<0.0001)$. The SCD disease community saw the promise of GLGE as a potential cure for the disease; however, many expressed concerns about misuse of the technology.

Conclusion: As discourse surrounding GLGE advances, it is crucial that we engage disease communities whose lives could be altered by these interventions in the policy conversations with researchers, bioethicists, and policymakers.

Funding: NIH, National Human Genome Research Institute, USA. Research Funding: ZIAHG200394.

B.M. Hollister: None. M. Gatter: None. K.E. Abdallah: None. A. Armsby: None. A.J. Buscetta: None. Y. Byeon: None. K.E. Cooper: None. S. Desine: None. M.J. Hahn: None. K.E. Ormond: None. V.L. Bonham: None.

\section{C07.4}

National implementation of genome-wide non-invasive prenatal testing as a first-tier screening test in the Netherlands: evaluation of women's perspectives

K. R. M. van der Meij ${ }^{1}$, M. N. Bekker ${ }^{2}$, J. T. Gitsels- van der Wal ${ }^{3}$ L. Martin ${ }^{3}$, E. H. van Vliet-Lachotzki ${ }^{4}$, D. R. M. Timmermans $^{5}$, R. H. Galjaard ${ }^{6}$, E. A. Sistermans ${ }^{1}$, L. Henneman ${ }^{1}$, Dutch NIPT Consortium

${ }^{1}$ Dept of Clinical Genetics, Amsterdam UMC, Vrije Universiteit Amsterdam, Amsterdam, Netherlands, ${ }^{2}$ Dept of Obstetrics and Gynaecology, Utrecht University Medical Center, Utrecht, Netherlands, ${ }^{3}$ Dept of Midwifery Science, 
Amsterdam UMC, Vrije Universiteit Amsterdam, Amsterdam, Netherlands, ${ }^{4}$ Dutch Genetic Alliance (VSOP), Soest, Netherlands, ${ }^{5}$ Dept of Public and Occupational Health, Amsterdam UMC, Vrije Universiteit Amsterdam, Amsterdam, Netherlands, ${ }^{6}$ Dept of Clinical Genetics, Erasmus University Medical Center, Rotterdam, Netherlands

The Netherlands is one of the first countries to offer NonInvasive Prenatal Testing (NIPT) within a governmentally supported screening program as a first-tier test for all pregnant women. Since April 2017, all pregnant women can choose between NIPT, First-trimester Combined Testing (FCT) (both $\sim € 175$ ) and no test, within the TRIDENT-2 study. Due to the favorable characteristics of NIPT there has been a strong demand for implementation. However, concerns have been raised regarding the impact on informed decision-making. This study evaluates women's perspectives on the offer of NIPT.

A nationwide questionnaire was completed by $n=752$ pregnant women, after receiving counseling for prenatal screening by a certified counselor but before testing, assessing: test preferences, attitudes, perceived pressure to test, knowledge and deliberation.

Of the respondents, $79 \%$ expressed a preference for NIPT, $2 \%$ for FCT, $17 \%$ no test and $2 \%$ were unsure. Main reason for preferring NIPT was: 'high accuracy compared to FCT'(29\%). Primary reason to refrain from testing was: 'every child is welcome' $(27 \%)$. Few women $(<5 \%)$ experienced societal or provider pressure (not) to test. Half (53\%) thought that children with Down syndrome were less accepted in society. Nearly all women $(97 \%)$ reported that they made a well-informed decision. Most women (94\%) had adequate prenatal screening knowledge $(\geq 4 / 7$ correct questions). Additional measures of informed choice will be presented.

The findings indicate that pregnant women experience little pressure to test, have adequate knowledge and a high level of perceived informed decision-making with NIPT offered within a governmentally supported screening program.

Grant: ZonMw Netherlands

K.R.M. van der Meij: None. M.N. Bekker: None. J.T. Gitsels- van der Wal: None. L. Martin: None. E.H. van Vliet-Lachotzki: None. D.R.M. Timmermans: None. R. H. Galjaard: None. E.A. Sistermans: None. L. Henneman: None.

\section{C07.5}

Implementing non-invasive prenatal testing (NIPT): An interview study of pregnant women's opinions about and experiences with societal pressure, reimbursement and an expanding scope
I. M. Bakkeren, A. Kater-Kuipers, E. M. Bunnik, A. T. J. I. Go, I. D. de Beaufort, R. H. Galjaard, S. R. Riedijk

\section{Erasmus Medical Centre, Rotterdam, Netherlands}

The non-invasive prenatal test (NIPT) as first trimester prenatal screening (FTS) for trisomies 21, 18 and 13 is offered to all pregnant women in the Netherlands. NIPT using whole genome sequencing allows for an expansion of the scope of FTS and the introduction of NIPT gives rise to ethical and societal concerns about deliberated decisionmaking, pressure to engage in screening and possible lack of equal access due to the financial contribution $(€ 175)$ to NIPT. We explored the opinions and experiences of pregnant women, who were offered FTS, about these concerns and about the possibility of a broadened scope. Nineteen pregnant women representing a diversity of backgrounds were interviewed using a semi-structured interview guide. Eight women $(42.1 \%)$ did not opt for prenatal screening and eleven $(57.9 \%)$ did $($ NIPT $=4$, combined test $=7$ ). Women experienced a free choice to accept or decline prenatal screening, despite sometimes receiving advice from others. Prior to pre-test counselling, some women had already deliberated about what an abnormal test result would mean to them. Others accepted or declined FTS without deliberation. The current Dutch policy of requiring a co-payment was acceptable to some, who believed it functioned as a threshold to think carefully about FTS. Others were concerned that a financial threshold would lead to unequal access to screening. Finally, pregnant women found it difficult to formulate opinions on the scope of FTS, because of lacking knowledge. Life expectancy, severity and treatability were considered important criteria for the inclusion of a condition in NIPT.

I.M. Bakkeren: None. A. Kater-Kuipers: None. E.M. Bunnik: None. A.T.J.I. Go: None. I.D. de Beaufort: None. R.H. Galjaard: None. S.R. Riedijk: None.

\section{C07.6}

The notion of "serious" for genetic disease: To qualify or not, that is the question

\section{E. Kleiderman ${ }^{1}$, V. Ravitsky ${ }^{2}$, B. Knoppers ${ }^{1}$ \\ ${ }^{1}$ Centre of Genomics and Policy, McGill University, Montreal, QC, Canada, ${ }^{2}$ Université de Montréal, Montreal, $Q C$, Canada}

Current advances and innovation in assisted reproductive technologies aim to promote the health and well-being of future children. They offer the possibility to select embryos with the greatest potential of being born healthy (e.g. preimplantation genetic testing) and may someday correct 
faulty genes responsible for heritable diseases in the embryo (e.g. human germline genome modification - HGGM). Most laws and policy statements surrounding HGGM refer to the notion of "serious" as a core parameter in determining what genetic conditions should be targeted by these technologies. The literature suggests that it is unrealistic to expect to define the concept universally, but that when employed it must be qualified and consider patient and physician perspectives. Yet, this notion remains undefined, rendering its application vague and decision-making subjective and arbitrary. We begin by presenting two general conceptions of 'disease' and 'health': the objectivist (i.e. based on biological facts) and constructivist (i.e. based on human values) frameworks. We then consider the rationale for a rights-based approach (e.g. right to science and right to the highest attainable health). The basic challenge is sorting out whether and to what extent social conditions have a role in helping to define what qualifies as a "serious" disease - and whether the classification of "serious" is flexible, depending on shifting social conditions and landscapes. Ultimately, decisions about what constitutes a "serious" condition would be contextualized to the individual but should also give appropriate weight and consideration to the broader implications for the notion of 'health' and 'disease' in society.

E. Kleiderman: None. V. Ravitsky: None. B. Knoppers: None.

\section{C08 Prenatal Genetics}

\section{C08.1}

Increased risk at first trimester screening: trisomies are not everything, but the risk for an atypical chromosome aberration is low. Experiences from the Swedish Pregnancy Register

\section{E. Iwarsson ${ }^{1}$, P. Conner ${ }^{2}$}

${ }^{1}$ Department of Molecular Medicine and Surgery, Clinical Genetics Unit, Karolinska Institutet and Karolinska University Hospital, Stockholm, Sweden, ${ }^{2}$ Center for Fetal Medicine, Karolinska Institutet and Karolinska University Hospital, Stockholm, Sweden

Introduction: This study aimed to investigate the detection rates of all types of chromosome aberrations if either NIPT or karyotype/array-CGH was used for further investigation in pregnancies with an increased risk $(\geq 1: 200)$ following combined first trimester screening (cFTS).

Materials and Methods: Retrospective review of 129 493 pregnancies examined during 2010-2017 in Stockholm county, including children born with a postnatally detected chromosome aberration.

Results: There were 872 (0.7\%) chromosome aberrations in the cohort. Common trisomies $(21,18$ and 13) or sex chromosome aberrations (SCA) constituted $86 \%$ of these and $14 \%$ were atypical chromosome aberrations (AChrA). If an invasive test were offered to the $5779(4.5 \%)$ women with an increased risk for a trisomy, $84 \%$ of all clinically significant chromosome aberrations and $55 \%$ of all AChrAs would be detected. In total $93 \%$ of the chromosome aberrations were detectable with NIPT (trisomies/SCAs). Investigating the $2323(1.8 \%)$ women with the highest risk $(\geq 1: 50)$ after cFTS, $27 \%$ had a chromosome aberration, $2.0 \%$ had an AChrA requiring an invasive test for diagnosis.

Conclusion: If only analysis for common trisomies and SCA is offered after cFTS, the residual risk for an AChrA in women with a risk $\geq 1: 200$ is $1.0 \%$ (55/5779). Taking into account the cases resulting in a miscarriage/IUFD and those detected due to malformation on ultrasound, the residual risk for a postnatal diagnosis of an AChrA is $0.7 \%$ (41/ 5779). That might be seen as a low residual risk, but looking from another angle 74\% (41/55) of the AChrAs will remain undetected using such an approach.

E. Iwarsson: None. P. Conner: None.

C08.2

The PREGCARE study: precision genetic counselling via personalised evaluation of recurrence risk for families with a child affected by a disorder caused by a de novo mutation

U. B. Abdullah ${ }^{1,2}$, M. Bernkopf $f^{1,2}$, N. Koelling ${ }^{1,2}$, S. J. McGowan ${ }^{3}$, J. Williams ${ }^{4}$, A. H. Németh ${ }^{5,6}$, H. Stewart ${ }^{6}$, P. Clouston ${ }^{4}$, A. O. M. Wilkie ${ }^{1,2}$, A. Goriely ${ }^{1,2}$

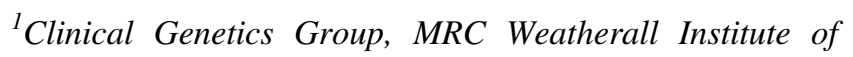
Molecular Medicine, University of Oxford, Oxford, United Kingdom, ${ }^{2}$ Nuffield Division of Clinical Laboratory Sciences, Radcliffe Department of Medicine, University of Oxford, Oxford, United Kingdom, ${ }^{3}$ Analysis, Visualisation and Informatics Group, MRC Weatherall Institute of Molecular Medicine, University of Oxford, Oxford, United Kingdom, ${ }^{4}$ Oxford Medical Genetics Laboratories, Oxford University Hospitals NHS Foundation Trust, The Churchill Hospital, Oxford, United Kingdom, ${ }^{5}$ Nuffield Department of Clinical Neurosciences, University of Oxford, Oxford, United Kingdom, ${ }^{6}$ Oxford Centre for Genomic Medicine, Oxford University Hospitals NHS Foundation Trust, Nuffield Orthopaedic Centre, Oxford, United Kingdom

While disorders caused by de novo mutations (DNMs) are usually assumed to be one-off events, in practice, the same mutation recurs in a subsequent pregnancy 
in 1-2\% of cases. However, this population-wide average does not reflect a family's individual risk: while most couples have a negligible risk, for a minority, the DNM is present in multiple parental gonadal cells (mosaicism) and the risk of a future offspring being affected is up to $50 \%$.

The PREGCARE (Precision Genetic Counselling and Reproduction) study aims to develop a systematic strategy to identify high-risk mosaic cases and provide families with a personalised assessment of transmission risk. Individual risk stratification is achieved through analysis of 14 tissue samples of various embryonic origin from the family trio (including a father's semen sample) using ultra-deep Illumina sequencing $(\sim 30,000 \mathrm{x})$ and DNM haplotype-phasing via Nanopore long-read-sequencing, to determine the parental origin.

We present data from the 20 first families to illustrate the power of this approach. While we detected instances of mosaicism, such as a maternally-originating KIF11 mutation $(\mathrm{VAF}=0.1-0.5 \%)$, for most families, the DNM was undetectable by deep-sequencing of parental samples (including sperm). Next, long-read-haplotyping permitted to single-out DNMs of paternal origin, confirming that these families have an extremely-low risk $(\leq 0.1 \%)$ of having another affected child.

Given our current understanding of mosaicism, this strategy should reassure up to $\sim 75 \%$ of couples that their recurrence risk is negligible. The ability to provide personalised transmission risk prior to conception is likely to impact upon clinical practice and family planning decisions.

U.B. Abdullah: None. M. Bernkopf: None. N. Koelling: None. S.J. McGowan: None. J. Williams: None. A. H. Németh: None. H. Stewart: None. P. Clouston: None. A.O.M. Wilkie: None. A. Goriely: None.

\section{C08.3}

\section{Validation of simultaneous detection of fetal} chromosome aneuploidy and monogenic diseases by a novel noninvasive prenatal testing method: Targeted And Genome-wide simultaneous sequencing (TAGs-seq)

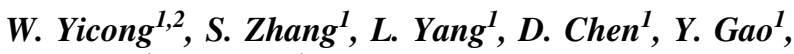 Y. Zhang ${ }^{1}$, F. Chen ${ }^{1}$}

${ }^{1}$ BGI-Shenzhen, Shenzhen, China, ${ }^{2}$ School of Bioscience and Bioengineering, South China University of Technology, Guangzhou, China

Introduction: Next generation sequencing (NGS)-based cell-free DNA (cfDNA) analysis has been widely adopted for non-invasive prenatal screening (NIPS) for fetal chromosome aneuploidy and monogenic diseases through different experiment strategies. We developed a new sequencing method embracing both advantages of targeted and genome-wide sequencing, so that it can simultaneously screen for fetal chromosomal aneuploidy and monogenic diseases in a noninvasive manner.

Methods: The novel method, called TAGs-seq, integrates a multiplex PCR step into the genome-wide NIPS library construction to simultaneously amplify whole genome and target region in one tube. After NGS, the genome-wide region displays a low sequencing depth $(0.1-0.5 \mathrm{X})$ and the target regions an ultra-high depth $(>1000 \mathrm{X})$, which were sufficient for detecting chromosome aneuploidy and singe base mutations, respectively.

Results: We validated in-blind in 517 plasma samples with previously confirmed outcomes of fetal. The TAGs-seq NIPS identified 23 cases of common aneuploidy, 9 cases of fetal de novo mutations, 27 cases of maternal monogenic diseases carrier and 458 cases of normal controls. Fetal results were concordant to the invasive diagnostic results, while maternal carrier results were concordant to sanger sequencing results.

Conclusion: We developed a novel TAGs-seq NIPS, which exploited whole-genome sequencing data and targeted sequencing data, providing a convenient, low-cost, and expandable solution to detect fetal chromosome aneuploidy and de novo mutations, as well as maternal carrier of monogenic diseases in a single experiment.

Details of positive cases detected by TAGs-seq in 517 prenant women plasma samples

Disease Mutation NO.

Fetal chromosomal aneuploidy

$\begin{array}{lll}\text { Down Syndrome } & \text { T21 } & 14 \\ \text { Edward Syndrome } & \text { T18 } & 5 \\ \text { Patau Syndrom } & \text { T13 } & 4\end{array}$

Fetal de novo monogenic disease

$\begin{array}{lll}\text { Achondroplasia } & \text { FGFR3 c.1138G }>\text { A } & 8 \\ \text { Achondroplasia } & \text { FGFR3 c.1118A }>\text { G } & 1\end{array}$

Maternal monogenic disease carrier

$\beta$ thalassemia $\quad$ HBB: c. $-78 \mathrm{~A}>\mathrm{G} \quad 1$

$\beta$ thalassemia $\quad$ HBB: c.316-197C $>\mathrm{T} \quad 1$

$\beta$ thalassemia $\quad$ HBB: c.216_217insA 1

Galactosemia $\quad$ GALT: c.940A $>\mathrm{G} \quad 7$

Wilson's disease $\quad$ ATP7B: c.3443T $>\mathrm{C} \quad 1$

Wilson's disease $\quad$ ATP7B: c. $2755 \mathrm{C}>\mathrm{G} \quad 4$

Wilson's disease $\quad$ ATP7B: c.2333G $>\mathrm{T} \quad 1$

Methylmalonic acidemia MUT: c. $1663 \mathrm{G}>\mathrm{A} \quad 1$

$\begin{array}{lll}\text { Cystic fibrosis } & \text { CFTR: c. } 2909 \mathrm{G}>\mathrm{A} & 1\end{array}$ 


\begin{tabular}{llr} 
Niemann-Pick Disease & NPC1: c.2073G $>A$ & 1 \\
Biotinidase Deficiency & BTD: c.1336G $>C$ & 1 \\
$\begin{array}{l}\text { Hereditary Hearing } \\
\text { Impairment }\end{array}$ & SLC26A4: IVS7-2A $>$ G 3 \\
$\begin{array}{l}\text { Hereditary Hearing } \\
\text { Impairment }\end{array}$ & GJB2: c.235 delC & 1 \\
$\begin{array}{l}\text { Hereditary Hearing } \\
\text { Impairment }\end{array}$ & SLC26: c.2027T>A & 2 \\
$\begin{array}{l}\text { Hereditary Hearing } \\
\text { Impairment }\end{array}$ & GJB3: c.538C $>$ T & 1 \\
\hline
\end{tabular}

W. Yicong: None. S. Zhang: None. L. Yang: None. D. Chen: None. Y. Gao: None. Y. Zhang: None. F. Chen: None.

\section{C08.4}

Systematic evaluation of prenatal and pediatric diagnostic yields from whole-genome sequencing in 8,954 individuals

C. Lowther ${ }^{1,2}$, H. Brand ${ }^{1,2}$, B. B. Currall, J. L. Giordano ${ }^{3}$, V. S. Aggarwal', H. Z. Whang', X. Zhao ${ }^{1,2}$, D. Lucente ${ }^{1}$, L. Margolin ${ }^{2}$, D. M. Werling ${ }^{5}$, J. Y. An ${ }^{5}$, S. Dong ${ }^{5}$, S. J. Sanders ${ }^{5}$, B. Devlin', K. Gilmore ${ }^{7}$, B. Powell ${ }^{8}$, A. Brandt ${ }^{9}$, A. H. O'DonnellLuria $^{1,2,10}$, N. J. Lennon ${ }^{2}$, D. B. Goldstein ${ }^{11}$, H. L. Rehm ${ }^{1,2}$, N. L. Vora ${ }^{7}$, D. G. MacArthur ${ }^{1,2}$, B. Levy ${ }^{4}$, R. Wapner $^{3}$, M. E. Talkowskil,2

${ }^{1}$ Center for Genomic Medicine, Boston, MA, United States, ${ }^{2}$ Program in Population and Medical Genetics and Genomics, The Broad Institute of M.I.T. and Harvard, Cambridge, MA, United States, ${ }^{3}$ Department of Obstetrics \& Gynecology, Columbia University Medical Center, New York, NY, United States, ${ }^{4}$ Department of Pathology and Cell Biology, Columbia University Medical Center, New York, NY, United States, ${ }^{5}$ Department of Psychiatry, UCSF Weill Institute for Neurosciences, University of California, San Francisco, San Francisco, CA, United States, ${ }^{6}$ Department of Psychiatry, University of Pittsburgh School of Medicine, Pittsburgh, PA, United States, ${ }^{7}$ Department of Obstetrics and Gynecology, Division of Maternal-Fetal Medicine, University of North Carolina at Chapel Hill, Chapel Hill, NC, United States, ${ }^{8}$ Center for Genomic Medicine Department of Genetics, School of Medicine, University of North Carolina at Chapel Hill, Chapel Hill, NC, United States, ${ }^{9}$ Department of Genetics, School of Medicine, University of North Carolina at Chapel Hill, Chapel Hill, NC, United States, ${ }^{10}$ Division of Genetics and Genomics, Boston Children's Hospital, Boston, MA, United
States, ${ }^{11}$ Institute for Genomic Medicine, Columbia University Medical Center, New York, NY, United States

Clinical genetic screening in prenatal and pediatric cohorts have traditionally required a decision to test targeted genes or mutational classes, as evaluation of all variant classes has been intractable. Whole genome sequencing (WGS) has the potential to transform diagnostic testing by capturing all classes variation with a single technology. Here, we compared diagnostic yields from WGS to those from karyotype, CMA, and whole exome sequencing (WES) in a pediatric cohort of 2,100 quartet families with a proband diagnosed with autism spectrum disorder (ASD; $\mathrm{n}=8,400$ ) and a prenatal cohort of 218 cases with a structural defect detected on ultrasound. We first benchmarked our bioinformatic pipelines on 519 ASD quartets, discovering 3.4M SNVs, 0.3M indels, and 5,863 structural variants (SVs) per genome. WGS recapitulated $99.6 \%$ of all CMA-predicted CNVs and $>97 \%$ of all de novo coding variants from WES. Molecular validation of 171 de novo SVs revealed a $97 \%$ confirmation rate. The yield from WGS exceeded all other technologies, but provided only $\sim 0.3 \%$ increased diagnostic yield over the combination of all conventional methods. We next evaluated WGS in the fetal structural anomaly samples and discovered a diagnostic variant in $9.8 \%$ of cases that had negative karyotype and CMA results. This study suggests a modest overall increased diagnostic yield of WGS compared to the combination of all conventional methods, and should temper enthusiasm regarding substantial increases in interpretable pathogenic variants from WGS. Nonetheless, WGS was superior to any individual method thus warranting evaluation as a first-tier screen in prenatal and pediatric diagnostic testing.

C. Lowther: None. H. Brand: None. B.B. Currall: None. J.L. Giordano: None. V.S. Aggarwal: None. H.Z. Whang: None. X. Zhao: None. D. Lucente: None. L. Margolin: None. D.M. Werling: None. J.Y. An: None. S. Dong: None. S.J. Sanders: None. B. Devlin: None. K. Gilmore: None. B. Powell: None. A. Brandt: None. A.H. O'Donnell-Luria: None. N.J. Lennon: None. D.B. Goldstein: None. H.L. Rehm: None. N.L. Vora: None. D. G. MacArthur: None. B. Levy: None. R. Wapner: None. M.E. Talkowski: None.

\section{C08.5}

Non-invasive prenatal diagnosis of sickle cell disease by next generation sequencing of cell-free DNA

J. C. van Campen ${ }^{1}$, L. Silcock ${ }^{2}$, M. Yau ${ }^{3}$, Y. Daniel',

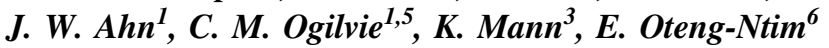

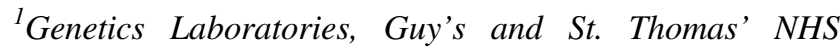
Foundation Trust, London, United Kingdom, ${ }^{2}$ Nonacus 
Ltd., Birmingham, United Kingdom, ${ }^{3}$ Viapath Genetics Laboratories, Guy's Hospital, London, United Kingdom, ${ }^{4}$ Viapath Haematological Sciences Laboratories, Guy's Hospital, London, United Kingdom, ${ }^{5}$ Department of Medical and Molecular Genetics, King's College, London, United Kingdom, ${ }^{6}$ Department of Women's Services, Guy's and St. Thomas' NHS Foundation Trust, London, United Kingdom

Introduction: Non-invasive prenatal diagnosis (NIPD) of paternally inherited and de novo autosomal dominant disorders is in diagnostic use in the UK. However, robust NIPD of autosomal recessive conditions has proven more challenging as this requires detection of fetal inheritance of a maternal allele from a mixed maternal-fetal pool of cell free DNA. The autosomal recessive disorder sickle cell disease is the most common referral indication for molecular genetic invasive prenatal diagnosis in the UK, however, NIPD is not yet available.

Methods: We use targeted next generation sequencing of cell-free DNA from maternal plasma to diagnose fetal sickle cell disease based on a relative mutation dosage approach. No paternal or proband samples are required. Unique molecular identifiers (UMIs) were incorporated into library preparation to enable accurate quantification of mutant and wildtype allele reads.

Results: 24 plasma samples from pregnant sickle cell disease carriers were analysed, of which 20 were concordant with the established genotype; two with low fetal fraction were inconclusive and two were discordant. In silico size selection of cfDNA fragments was found to enhance the fetal fraction for all samples, and modifications to UMI capture improved diagnostic accuracy. Samples from as early as eight weeks gestation were successfully genotyped.

Conclusion: We demonstrate that NIPD for sickle cell disease is approaching clinical utility. Other autosomal recessive disorders may benefit from a similar approach.

This work was funded by the Guy's and St. Thomas' Hospital Charity.

J.C. van Campen: None. L. Silcock: A. Employment (full or part-time); Significant; Nonacus Ltd.. E. Ownership Interest (stock, stock options, patent or other intellectual property); Significant; Nonacus Ltd.. M. Yau: None. Y. Daniel: None. J.W. Ahn: None. C.M. Ogilvie: None. K. Mann: None. E. Oteng-Ntim: None.

\section{C08.6}

Prevalence and clinical outcome of mosaicism in uncultured chorionic villus samplings after chromosomal microarray
I. Lund $^{1,2,3}$, N. Becher ${ }^{1,2,3}$, E. Vestergaard ${ }^{2}$, R. Christensen ${ }^{1,2}$, O. Petersen ${ }^{2,4,5}$, E. Steffensen ${ }^{2}$, I. Vogel ${ }^{1,2,3}$

${ }^{1}$ Department of Clinical Genetics, Aarhus University Hospital, Aarhus N., Denmark, ${ }^{2}$ Center for Fetal Diagnostics, Aarhus University Hospital/Aarhus University, Aarhus N, Denmark, ${ }^{3}$ Department of Biomedicine, Aarhus University, Aarhus C, Denmark, ${ }^{4}$ Department of Gynecology and Obstetrics, Fetal Medicine Unit, Aarhus University Hospital, Aarhus N, Denmark, ${ }^{5}$ Department of Clinical Medicine, Aarhus University, Aarhus C, Denmark

Objective: To evaluate the prevalence and clinical outcome of mosaicism in uncultured chorionic villus samplings (CVS) using chromosomal microarray (CMA).

Materials and Methods: We retrieved all results of chromosomal microarray after chorionic villus sampling from January $1^{\text {st }} 2011$ to November $30^{\text {th }} 2017$ registered in our local laboratory database. Mosaic results from uncultured chorionic villus samples and follow-up with amniocenteses, fetal tissue or postnatal blood were studied and matched with clinical data from The Danish Fetal Medicine Database.

Results: The prevalence of mosaicism in CVS was 93/ $2,288,4.1 \%$ (95\%CI: $3.3-5.0 \%)$ of which 16 cases $(17.2 \%$, 95\% CI: $10.5-26.7 \%)$ concerned submicroscopic copy number variations $(\mathrm{CNVs})<10$ megabases $(\mathrm{Mb})$. Follow-up analysis was performed in 62 cases. True fetal mosaicism (TFM) was confirmed in $7 / 38,18.4 \%$, when mosaicism involved whole chromosome aneuploidy and in 6/24 cases, $25.0 \%$, when involving a CNV $(p=0.59)$. Mean birth weight $\mathrm{z}$-score was higher in cases of confined placental mosaicism for a CNV $(0.21)$ than cases involving whole chromosomes $(-0.74)(p=0.02)$.

Conclusion: Prevalence of mosaicism in CVS is significantly higher after CMA on uncultured cells than after conventional karyotyping. The risk of TFM is equally high in cases of mosaicism for CNVs and whole chromosomes.

I. Lund: None. N. Becher: None. E. Vestergaard: None. R. Christensen: None. O. Petersen: None. E. Steffensen: None. I. Vogel: None.

\section{C09 Cancer genetics}

\section{C09.1}

Germline genetic variation drives the somatic landscape of tumors

N. Zaitlen ${ }^{1}$, S. Mangul ${ }^{1}$, A. Gusev ${ }^{2}$ 
${ }^{1}$ UCLA, LA, CA, United States, ${ }^{2}$ DFCI, Boston, MA, United States

Hundreds of thousands of cancer patients have had their tumors sequenced to identify clinically actionable mutations. This activity has produced valuable research data leading to significant discoveries in basic and translational domains. Unfortunately, the targeted nature of tumor sequencing precludes powerful interrogation of germlinesomatic interactions. To address this shortcoming we developed a computational approach to infer genome-wide germline variation, including HLA, TCR/BCR, and microbial sequences, from off-target sequencing reads. We applied our method to the DFCI PROFILE cohort ( $>20,000)$, producing the world's largest germlinesomatic data set by over an order of magnitude. Levering this resource, we test for associations between germline and somatic variation. Specifically, for the mutational burden in each PROFILE target gene as well for total mutational burden (TMB), we conduct association, heritability, population prevalence, and polygenic risk score association tests. Our completed analysis of the lung adenocarcinoma (LUAD) sub-cohort has already produced strongly significant associations between germline and somatic events. We identify over forty significant germline associations (pvalue $<5 \mathrm{e}-8$ ) to mutations in oncogenes including BRCA1, ALK, DNMT3A, EPHA3/5, GLI2, as well total mutational burden. We estimate heritability over $60 \%$ for multiple genes including BRCA1, EPHA3/5. We show that asian genetic ancestry is associated with EGFR and KRAS mutations. Finally, we show that polygenic risk for lung cancer subtypes is associated to mutations in specific genes and total mutational burden. These results demonstrate that germline variation is a major contributor to the dynamics of somatic mutations and cancer outcomes.

N. Zaitlen: None. S. Mangul: None. A. Gusev: None.

\section{C09.2}

Germline TP53 mutations: the predominant genetic cause of adrenocortical carcinoma

M. Renaux-Petel ${ }^{1,2}$, F. Charbonnier ${ }^{1}$, I. Tournier ${ }^{\text {I, }}$, G. Lienard ${ }^{I}$, J. Bou ${ }^{I}$, E. Kasper ${ }^{I}$, B. Leheup ${ }^{3}$, L. Mansuy ${ }^{4}$, L. Guerrini-Rousseau ${ }^{5}$, L. Brugières ${ }^{5}$, A. Liard-Zmuda ${ }^{2}$, S. Baert-Desurmont ${ }^{1}$, T. Frebourg ${ }^{I}$, G. Bougeard ${ }^{1}$

${ }^{1}$ Department of Genetics, Normandy Centre for Genomic and Personalized Medicine, Normandy University, UNIROUEN, Inserm U1245 and Rouen University Hospital, Rouen, France, ${ }^{2}$ Department of Child and Adolescent Surgery, Rouen University Hospital, Rouen, France, ${ }^{3}$ Department of Clinical Genetics, Nancy University Hospital, Nancy, France, ${ }^{4}$ Department of Paediatric
Oncology, Nancy University Hospital, Nancy, France, ${ }^{5}$ Child and adolescent cancer Department, Gustave Roussy Cancer Campus, Villejuif, France

Adrenocortical carcinoma (ACC) is a rare aggressive tumour that can be associated with Li-Fraumeni syndrome (LFS), resulting from germline TP53 mutations. The aim of this study was to establish the frequency of germline TP53 alterations in ACC from a large series of 147 French patients (98 children, 49 adults). Sanger sequencing and QMPSF screening for genomic rearrangements revealed a deleterious germline TP53 alteration in 53 cases (36\%), among which only $32(60 \%)$ had a familial history suggestive of LFS. Re-analysis of 55 negative cases, using NGS performed at high depth, subsequently identified 2 additional cases of mosaic mutations. The mutation detection rate was $49 \%$ in children and $14 \%$ in adults, and reached $72 \%$ in female patients presenting with an ACC before 2 years of age. Among 16 ACC patients treated for a secondary tumour, 12 carried a germline TP53 alteration. p. (Arg158His) and p.(Arg337His) mutations were recurrent and the majority of the alterations were missense mutations without dominant-negative effect. This contrasts with the general distribution of germline TP53 alterations characterized by the predominance of dominant-negative missense mutations with higher penetrance and confirms that most of the germline TP53 alterations detected in ACC patients are associated with a lower penetrance. This study shows that germline TP53 alterations are the main cause of ACC, in particular in childhood, which is probably explained by a critical role of p53 in the development of the adrenal cortex. These data justify ensuring an exhaustive analysis of TP53 in all ACC cases, independently of the familial history.

M. Renaux-Petel: None. F. Charbonnier: None. I. Tournier: None. G. Lienard: None. J. Bou: None. E. Kasper: None. B. Leheup: None. L. Mansuy: None. L. Guerrini-Rousseau: None. L. Brugières: None. A. LiardZmuda: None. S. Baert-Desurmont: None. T. Frebourg: None. G. Bougeard: None.

\section{C09.3}

Cell free-DNA pinpoints specific clonal expansion at disease progression in solid cancers

\author{
M. Palmieri', M. Baldassarri', F. Fava ${ }^{1,2}$, A. Fabbiani, ${ }^{1,2}$, \\ E. Gelli, R. Tita ${ }^{2}$, P. Torre ${ }^{3}$, R. Petrioli, \\ T. Hadijstilianou ${ }^{4}$, D. Galimberti ${ }^{4}$, E. Cinotti ${ }^{5}$, \\ M. Mencarelli ${ }^{2}$, A. Pinto ${ }^{2}$, S. Marsili ${ }^{3}$, E. Frullanti ${ }^{\text {, }}$, \\ A. Renieri ${ }^{1,2}$
}

${ }^{1}$ Medical Genetics, University of Siena, Siena, Italy, ${ }^{2}$ Genetica Medica, Azienda Ospedaliera Universitaria Senese, Siena, Italy, ${ }^{3}$ Medical Oncology, Azienda 
Ospedaliera Universitaria Senese, Siena, Italy, ${ }^{4}$ Department of Ophthalmology, Referral Center for Retinoblastoma, Azienda Ospedaliera Universitaria Senese, Siena, Italy, ${ }^{5}$ Department of Medical, Surgical and NeuroSciences, Dermatology Unit, University of Siena, Siena, Italy

Ten different solid tumors were selected in order to identify clones escaping standard treatments. In a cohort of 39 patients, next generation sequencing of 52 cancer-driver genes on cell free-DNA was able to pick up clones responsible for disease progression in $60 \%$ of cases. Each tumor had a mean of 1,3 mutated genes with 1-3 range. Disease progression was associated with an increased levels of cfDNA. Point mutations in TP53, PIK3CA, and CNV in FGFR3 were among the most responsible, with a rate of 41 , 16 , and $13 \%$, respectively. Increased CNV of FGF receptors were identified in addition to non small cell adenocarcinoma of lung also in pancreatic, gastric and cholangiocarcinoma. Other clones had mutation in ESR1 (breast), CTNNB1 (uterus), KRAS and CCND2 (pancreas), EGFR and BRAF (lung). Retinoblastoma cases not responsible to repeated cycles of intraocular Melphalan showed expanding mutated clones in PTEN or SMAD4. These results show that irrespective to tumor mutational burden at origin and subsequent complex clonal evolution, a simplified mutational load is present at disease progression. One or few "sniper" clones drive progression and the molecular profile has a weak correlation with the primary tumor. Single driver mutations in TP53 remain the main target of a not yet developed specific therapy in most tumors such as breast, ovarian, uterine, lung, gastric cancers and glioblastoma. Among the actionable mutations, PIK3CA were found not only in breast cancers but also in uterine carcinoma, Sezary syndrome and glioblastoma, pinpointing the needs of specific trials in these tumors.

M. Palmieri: None. M. Baldassarri: None. F. Fava: None. A. Fabbiani: None. E. Gelli: None. R. Tita: None. P. Torre: None. R. Petrioli: None. T. Hadijstilianou: None. D. Galimberti: None. E. Cinotti: None. M. Mencarelli: None. A. Pinto: None. S. Marsili: None. E. Frullanti: None. A. Renieri: None.

\section{C09.4}

Molecular classification of B-other pediatric B-cell precursor acute lymphoblastic leukemia by DNA methylation and RNA-sequencing

Y. Marincevic-Zuniga ${ }^{1}$, S. Nystedt ${ }^{1}$, S. Nilsson ${ }^{1}$, J. Almlöf ${ }^{1}$, H. Lilljebjörn ${ }^{2}$, T. Fioretos ${ }^{2}$, T. Flaegstad ${ }^{3}$, U. Norén-Nyström ${ }^{4}$, M. Heyman ${ }^{5}$, K. Schmiegelow ${ }^{6}$, J. Kanerva ${ }^{7}$, G. Lönnerholm ${ }^{8}$, J. Nordlund ${ }^{1}$
${ }^{1}$ Molecular Medicine and Science for Life Laboratory, Uppsala University, Uppsala, Sweden, ${ }^{2}$ Department of Laboratory Medicine, Lund University, Lund, Sweden, ${ }^{3}$ Department of Pediatrics, Troms $\phi$ University and University Hospital, Tromsø, Norway, ${ }^{4}$ Department of Clinical Sciences, Pediatrics, Umeå University, Umeå, Sweden, ${ }^{5} 5$ Childhood Cancer Research Unit, Karolinska Institutet, Astrid Lindgren Children's Hospital, Karolinska University Hospital, Stockholm, Sweden, ${ }^{6}$ Pediatrics and Adolescent Medicine, Rigshospitalet, and the Medical Faculty, Institute of Clinical Medicine, University of Copenhagen, Copenhagen, Denmark, ${ }^{7}$ Division of Hematology-Oncology and Stem Cell Transplantation, Children's Hospital, Helsinki University Central Hospital and University of Helsinki, Helsinki, Finland, ${ }^{8}$ Department of Women's and Children's Health, Uppsala University, Uppsala, Sweden

Introduction: Recurrent chromosomal alterations in pediatric B-cell precursor acute lymphoblastic leukemia (BCPALL) are important markers for cytogenetic subgrouping and risk-directed therapy stratification. Many of these alterations are strongly associated with specific gene expression and DNA methylation patterns that can be used for ALL subtype classification. Amongst the Nordic BCPALL cases diagnosed between 1996 and 2012, subtypedefining aberrations were not detected at diagnosis in approximately $25 \%$ of cases, representing a large group of uncharacterized cases collectively referred to as B-other.

Materials and Methods: We designed and applied DNA methylation classifiers as a tool for subtype discovery in 1142 Nordic ALL patients including 284 patients with Bother phenotype. Classifiers were designed for 10 recurrent ALL subtypes including the recently described DUX4- and ZNF384-rearranged BCP-ALL subtypes. The classification results were validated by RNA-sequencing for fusion gene detection and copy number analysis.

Results: DNA methylation classification successfully assigned an unambiguous new subtype to $>50 \%$ of the 284 patients in the B-other group. With RNA-sequencing, we verified the DNA methylation result by identifying fusion genes involving $D U X 4, Z N F 384$, or MEF2D genes, fusions common in the BCR-ABL1-like and ETV6-RUNX1-like groups, and fusion genes/mutations affecting PAX5. Furthermore, we show the clinical relevance of these new groups in Nordic ALL patients by differing event free and overall survival rates.

Conclusion: DNA methylation classification is a powerful tool for subtype discovery that in combination with RNA-sequencing and copy number analysis enables improved delineation of the genetic alterations of previously uncharacterized BCP-ALL patients.

Y. Marincevic-Zuniga: None. S. Nystedt: None. S. Nilsson: None. J. Almlöf: None. H. Lilljebjörn: None. T. 
Fioretos: None. T. Flaegstad: None. U. Norén-Nyström: None. M. Heyman: None. K. Schmiegelow: None. J. Kanerva: None. G. Lönnerholm: None. J. Nordlund: None.

\section{C09.5}

Polygenic risk scores modify age-dependent breast cancer risk in $\mathrm{CHEK} 2$ germline mutation carriers

J. Borde ${ }^{1}$, C. Ernst ${ }^{1}$, K. Weber-Lassalle ${ }^{1}$, D. Niederacher ${ }^{2}$, J. Hauke ${ }^{1}$, J. Horváth ${ }^{3}$, N. Weber-Lassalle ${ }^{1}$, A. Meindl, E. Pohl-Rescigno ${ }^{1}$, N. Arnold ${ }^{5}$, A. Lee Le C. Engel $^{7,8}$, B. Wappenschmidt ${ }^{1}$, M. Schmidt ${ }^{9}$, A. Antoniou ${ }^{6}$, R. K. Schmutzler ${ }^{1}$, K. Kuchenbäcker ${ }^{10,11}$, E. Hahnen ${ }^{1}$

${ }^{1}$ Center for Hereditary Breast and Ovarian Cancer, Center for Integrated Oncology (CIO), University of Cologne, Faculty of Medicine and University Hospital Cologne, Cologne, Germany, ${ }^{2}$ Department of Gynaecology and Obstetrics, University Hospital Duesseldorf, HeinrichHeine University Duesseldorf, Duesseldorf, Germany, ${ }^{3}$ Institute for Human Genetics, University Hospital Muenster, Muenster, Germany, ${ }^{4}$ Department of Gynecology and Obstetrics, LMU Munich, University Hospital Munich, Munich, Germany, ${ }^{5}$ Institute of Clinical Molecular Biology, Department of Gynaecology and Obstetrics, University Hospital of Schleswig-Holstein, Campus Kiel, ChristianAlbrechts University Kiel, Kiel, Germany, ${ }^{6}$ Centre for Cancer Genetic Epidemiology, Department of Public Health and Primary Care, University of Cambridge, Cambridge, United Kingdom, ${ }^{7}$ Institute for Medical Informatics, Statistics and Epidemiology, University of Leipzig, Leipzig, Germany, ${ }^{8}$ LIFE ᄀ- Leipzig Research Centre for Civilization Diseases, University of Leipzig, Leipzig, Germany, ${ }^{9}$ Division of Molecular Pathology, Netherlands Cancer Institute, Amsterdam, Netherlands, ${ }^{10}$ Division of Psychiatry, University College London, Maple House, 149 Tottenham Court Road, London, United Kingdom, ${ }^{11} \mathrm{UCL}$ Genetics Institute, University College London, Gower Street, London, United Kingdom

CHEK2 germline mutations are associated with a $20-30 \%$ lifetime risk for breast cancer (BC). It is suggested that additional genetic factors, such as BC-associated SNPs identified by GWAS, modify individual BC risks. For BRCA1/2 mutation carriers, the combined effects of BCassociated SNPs (polygenic risk score, PRS) have been shown to be informative for individual risk-stratification. The question arises whether PRS-based risk-stratification is applicable for $C H E K 2$ mutation carriers. We analyzed 685 female $C H E K 2$ germline mutation carriers (464 c. 1100 delC and 143 carriers of other protein-truncating variants, 78 individuals with pathogenic missense variants). A total of
573 mutation carriers were affected by BC (474 unilateral $\mathrm{BC}, 99$ bilateral BC), with a mean age at first/secondary diagnosis of 45.7 (23-84)/49.2 (31-79) years, respectively. 112 mutation carriers were not affected by BC (mean age at last follow-up: 53.1 years). Study design: A cohort approach including retrospective and prospective events was applied. Recruitment was through genetic counselling centers including index patients and relatives. Genotyping of 77 BC-associated SNPs (Mavaddat et al., 2015) was performed by Fluidigm ${ }^{\circledR}$ Access Array and NGS. PRScalculation was performed according to Kuchenbaecker et al., 2017. Kaplan-Meier analysis revealed that mutation carriers within the highest PRS decile reported a significantly earlier age at first onset compared with patients within the lowest decile $(\mathrm{P}=5 \mathrm{e}-06)$. Patients within the highest decile show a mean age at first diagnosis of 43.6 (23-69) years vs. 48.7 (27-65) years in the lowest decile $(\mathrm{p}=0.007)$. Thus, combined genotyping of BC-associated SNPs may improve personal risk prediction for $C H E K 2$ mutation carriers.

J. Borde: None. C. Ernst: None. K. Weber-Lassalle: None. D. Niederacher: None. J. Hauke: None. J. Horváth: None. N. Weber-Lassalle: None. A. Meindl: None. E. Pohl-Rescigno: None. N. Arnold: None. A. Lee: None. C. Engel: None. B. Wappenschmidt: None. M. Schmidt: None. A. Antoniou: None. R.K. Schmutzler: None. K. Kuchenbäcker: None. E. Hahnen: None.

\section{C09.6}

Application of genomics and cognitive technology in precision oncological medicine

I. Sánchez Guiu', D. Cantalapiedra ${ }^{1}$, V. Felipe-Ponce ${ }^{I}$, S. Santillán ${ }^{1}$, J. García ${ }^{2}$, S. Lois ${ }^{2}$, J. Triviñón R. Miñambres ${ }^{3}$, B. Cortina ${ }^{3}$, M. Vázquez San Antonio ${ }^{4}$, C. Collado Micó ${ }^{4}$, V. Fernández Pedrosa ${ }^{1}$, C. RodriguezAntona $^{5}$, O. Calvete ${ }^{6}$, A. Gonzalez Neira ${ }^{7}$, C. MartínezLaperche $^{8}$, I. Buño Bordeh ${ }^{8}$, L. Rodríguez Rojas ${ }^{9}$, A. Zambrano ${ }^{10}$, J. Escobar ${ }^{11}$, J. Falla ${ }^{12}$, N. Tolaba ${ }^{13}$, P. Bazzoni ${ }^{13}$, M. Montero Alvi ${ }^{13}$, E. de Álava ${ }^{14}$, D. Azuara ${ }^{15}$, J. Ruffinelli ${ }^{15}$, M. Varela ${ }^{15}$, E. Nadal ${ }^{15}$, C. Lázaro ${ }^{15}$, C. Moya ${ }^{1}$, G. Ribas ${ }^{1}$

${ }^{1}$ Medical Genetics Unit, Sistemas Genómicos, Valencia, Spain, ${ }^{2}$ Bioinformatics Department, Sistemas Genómicos, Valencia, Spain, ${ }^{3} I+D+i$ Department, Sistemas Genómicos, Valencia, Spain, ${ }^{4}$ Next-Generation Sequencing Laboratory, Sistemas Genómicos, Valencia, Spain, ${ }^{5}$ Hereditary Endocrine Cancer Group, Human Cancer Genetics Programme, Spanish National Cancer Research Centre (CNIO), Madrid, Spain, ${ }^{6}$ Human Genetics Group, Spanish National Cancer Research Center (CNIO), Madrid, Spain, ${ }^{7}$ Human Genotyping Unit-CeGen, Human Cancer Genetics Programme, 
Spanish National Cancer Research Centre (CNIO), Madrid, Spain, ${ }^{8}$ Gregorio Marañón Health Research Institute (IiSGM), Madrid, Spain, ${ }^{9}$ Genetics Service, Fundación Valle del Lili, Cali, Colombia, ${ }^{10}$ Oncology Service, Fundación Valle del Lili, Cali, Colombia, ${ }^{11}$ Pathology Department, Fundación Valle del Lili, Cali, Colombia,

${ }^{12}$ Research Centre, Fundación Valle del Lili, Cali, Spain,

${ }^{13}$ Pathology and Genetics Anatomy Program, Arturo Oñativia Hospital, Salta, Argentina, ${ }^{14}$ Anatomical Pathology, Virgen del Rocio Hospital, Sevilla, Spain, ${ }^{15}$ Catalan Institute of Oncology, Duran I Reynals Hospital, Barcelona, Spain

Introduction: Precision medicine unifies clinical, anatomopathological and molecular data, and gives a biological profile tumor for each patient, favoring not only the diagnosis but giving personalized treatment alternatives and monitoring disease progression. New technologies are necessary in order to overcome these challenges. This study tested the robustness and feasibility of such technology, called IBM Watson for Genomics (WFG), which uses natural language processing to obtain evidence-based targeted therapy and clinical trial options in an automated manner.

Material and Methods: 39 different types of cancer in different stages (colon, lung, gastric, myeloid leukemia, angiosarcoma, bone, thyroid and renal sarcoma) were sequenced using different options of NGS panels (Trusight-170, LMA-NEOPLASMeprofile, Oncominefusion-assay and Archer-FusionPlex) on the HiSeq/ MiSeq and S5-XL platforms of IonTorrent. The resulting data was interpreted by WFG. Results: WFG detected actionable alterations in 28 tumors. In $17 \%$ of cases WFG agreed with the treatment applied by the oncologists or detected resistance to drugs in patients who did not respond to treatment. $64 \%$ of patients whose first line treatment failed, WFG detected alternative therapies and classified patients as candidates for clinical trials and/or for those pending approval. In oncohaematological tumors, it was possible to evaluate the genomic profile throughout the disease (diagnosis, relapse and recurrence) as well as its clonal evolution. This approach allowed the selection of drugs that led to the maximum beneficial effect.

Conclusion: Genetic characterization of tumors through NGS, complemented by evidence-based therapeutic intervention options, provides accurate molecular diagnosis, tumor sub-classification and treatment options. Additionally, this strategy offers new information to evaluate patients.

I. Sánchez Guiu: None. D. Cantalapiedra: None. V. Felipe-Ponce: None. S. Santillán: None. J. García: None. S. Lois: None. J. Triviño: None. R. Miñambres: None. B.
Cortina: None. M. Vázquez San Antonio: None. C. Collado Micó: None. V. Fernández Pedrosa: None. C. Rodriguez-Antona: None. O. Calvete: None. A. Gonzalez Neira: None. C. Martínez-Laperche: None. I. Buño Bordeh: None. L. Rodríguez Rojas: None. A. Zambrano: None. J. Escobar: None. J. Falla: None. N. Tolaba: None. P. Bazzoni: None. M. Montero Alvi: None. E. de Álava: None. D. Azuara: None. J. Ruffinelli: None. M. Varela: None. E. Nadal: None. C. Lázaro: None. C. Moya: None. G. Ribas: None.

\section{C10 Cardiovascular disorders}

\section{C10.1}

Sequence variants associated with resistant hypertension implicate mechanisms affecting potassium levels

V. Tragante ${ }^{I}$, P. Sulem ${ }^{I}$, G. Thorleifsson ${ }^{1}$, M. L. Frigge ${ }^{I}$, J. G. Arthur ${ }^{2}$, F. W. Asselbergs ${ }^{3}$, D. C. Crawford ${ }^{4}$, A. M. Deaton ${ }^{1}$, G. I. Eyjolfsson ${ }^{5}$, S. Gretarsdottir ${ }^{\text {, }}$, G. H. Halldorsson ${ }^{1}$, A. Helgadottir ${ }^{1}$, I. Jonsdottir ${ }^{1}$, R. P. Kristjansson ${ }^{1}$, P. Melsted ${ }^{1}$, A. Oddson ${ }^{1}$, I. Olafsson ${ }^{6}$, R. Palsson ${ }^{6}$, O. Sigurdardottir ${ }^{7}$, E. Sigurdsson ${ }^{8}$, J. K. Sigurdsson ${ }^{1}$, G. Sveinbjornsson ${ }^{1}$, G. Masson ${ }^{1}$, D. O. Arnar ${ }^{\text {, G. Ghorgeirsson }}{ }^{1}, U$. Thorsteinsdottir ${ }^{1}$, D. F. Gudbjartsson ${ }^{1}$, H. Holm ${ }^{1}$, K. Stefansson ${ }^{1}$

${ }^{1}$ deCODE Genetics/Amgen, Reykjavik, Iceland, ${ }^{2} 10 x$ Genomics, Pleasanton, CA, United States, ${ }^{3}$ University Medical Center Utrecht, Utrecht, Netherlands, ${ }^{4}$ Case Western Reserve University, Cleveland, OH, United States, ${ }^{5}$ The Laboratory in Mjodd, Reykjavik, Iceland, Reykjavik, Iceland, ${ }^{6}$ Landspitali-The National University Hospital of Iceland, Reykjavik, Iceland, ${ }^{7}$ Akureyri Hospital, Akureyri, Iceland, ${ }^{8}$ University of Iceland, Reykjavik, Iceland

Resistant hypertension (rHTN) is a severe form of hypertension associated with marked cardiovascular morbidity. We performed a genome-wide association study of 14,756 rHTN cases using 24,006 controlled hypertensives as controls (cHTN), both defined based on drug prescription and blood pressure data from Iceland, the UK Biobank and eMERGE. We found four genome-wide significant rHTN loci, all known blood pressure loci, and identified eight more rHTN loci using reported blood pressure associations as a prior. Several of the strongest hypertension variants do not show association with rHTN while others increase the risk of rHTN more than that of hypertension. Parent-oforigin analysis shows increased risk of rHTN associated with transmission by the father for three variants (rs880315 near CASZ1, rs569550 near LSPI and rs1006096 near 
RXFP2). Nine of the twelve rHTN variants associate with blood potassium levels, with the risk allele associating with lower potassium levels, and the potassium effect of blood pressure variants predicts their association with rHTN beyond their blood pressure effect. Furthermore, a genetic risk score of the rHTN variants associates with hyperaldosteronism. We also observed that, before therapy, individuals with rHTN have lower potassium levels than both normotensives and those with controlled hypertension. The most significant rHTN variant is in KCNK3, a potassium channel gene that has been associated with pulmonary arterial hypertension in men and hyperaldosteronism in mice. In conclusion, we show genetic differences between hypertension and rHTN and a prominent role of mechanisms affecting potassium homeostasis for development of rHTN, implicating mineralocorticoid-related pathways, aldosterone in particular.

V. Tragante: A. Employment (full or part-time); Significant; deCODE Genetics. P. Sulem: A. Employment (full or part-time); Significant; deCODE Genetics. G. Thorleifsson: A. Employment (full or part-time); Significant; deCODE Genetics. M.L. Frigge: A. Employment (full or part-time); Significant; deCODE Genetics. J.G. Arthur: A. Employment (full or part-time); Significant; 10x genomics. F.W. Asselbergs: None. D.C. Crawford: None. A.M. Deaton: A. Employment (full or part-time); Significant; Amgen. G.I. Eyjolfsson: None. S. Gretarsdottir: A. Employment (full or part-time); Significant; deCODE Genetics. G.H. Halldorsson: A. Employment (full or parttime); Significant; deCODE Genetics. A. Helgadottir: A. Employment (full or part-time); Significant; deCODE Genetics. I. Jonsdottir: A. Employment (full or part-time); Significant; deCODE Genetics. R.P. Kristjansson: A. Employment (full or part-time); Significant; deCODE Genetics. P. Melsted: A. Employment (full or part-time); Significant; deCODE Genetics. A. Oddson: A. Employment (full or part-time); Significant; deCODE Genetics. I. Olafsson: None. R. Palsson: None. O. Sigurdardottir: None. E. Sigurdsson: None. J.K. Sigurdsson: A. Employment (full or part-time); Significant; deCODE Genetics. G. Sveinbjornsson: A. Employment (full or parttime); Significant; deCODE Genetics. G. Masson: A. Employment (full or part-time); Significant; deCODE Genetics. D.O. Arnar: A. Employment (full or part-time); Significant; deCODE Genetics. G. Thorgeirsson: A. Employment (full or part-time); Significant; deCODE Genetics. U. Thorsteinsdottir: A. Employment (full or part-time); Significant; deCODE Genetics. D.F. Gudbjartsson: A. Employment (full or part-time); Significant; deCODE Genetics. H. Holm: A. Employment (full or parttime); Significant; deCODE Genetics. K. Stefansson: A. Employment (full or part-time); Significant; deCODE Genetics.
C10.2

Multi-omics approach identifies three novel genes for bicuspid aortic valve related aortopathy

\section{Luyckx', A. A. Kumar', E. Gillis ${ }^{\text {I, R. A. Gould }}$, H. Aziz ${ }^{2}$, C. E. Woods ${ }^{2}$, M. A. Seman-Senderos ${ }^{2}$, G. MacCarrick ${ }^{2}$, E. Sparks ${ }^{2}$, A. S. MacCallion ${ }^{2}$, L. Van

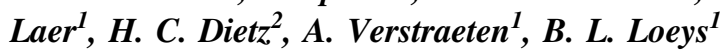

${ }^{1}$ Center for Medical Genetics, Edegem, Belgium, ${ }^{2}$ McKusick-Nathans Institute of Genetic Medicine, Johns Hopkins University School of Medicine, Baltimore, MD, United States

Bicuspid aortic valve (BAV) is the most common congenital heart defect (CHD), affecting $1-2 \%$ of the population. Although the associated development of thoracic aortic aneurysms (TAAs) was historically attributed to altered blood flow, high heritability (89\%) suggests a strong genetic contribution. Incomplete penetrance and variable clinical expression hampered gene identification. The only established BAV/TAA-gene is NOTCHI, accountable for less than $1 \%$.

Combined approach of copy number variation (CNV) analysis, resequencing of candidate genes and exome sequencing of families identified three novel candidate genes which were further validated in a large BAV/TAAcohort $(n=637)$.

$\mathrm{CNV}$ analysis revealed a $89 \mathrm{~kb}$ duplication upstream of the T-box transcription factor TBX20. Fine-mapping of the duplication confirmed involvement of a TAD-boundary and two of nine region-overlapping Decipher patients, carrying a bigger deletion, presented with syndromic CHD. After resequencing, variant burden analysis strengthened the genetic evidence for TBX2O involvement in BAV/TAA $(1.1 \% ; \mathrm{p}=0.03)$.

Targeted resequencing of 22 candidate genes unveiled SMAD6 as the most common BAV/TAA-gene $(2.5 \%$; $\mathrm{p}=$ 0.002). Investigation of an additional cohort of 473 TAApatients, demonstrated that although only $14 \%$ also presented BAV, all novel likely pathogenic SMAD6 variants $(\mathrm{n}=7)$ were identified in BAV/TAA individuals, further consolidating the role of SMAD6 variants to the BAV/TAAaetiology.

Finally, exome sequencing discovered a $\mathrm{ROBO} 4$ splicesite mutation segregating in a BAV/TAA-family. Resequencing revealed enrichment for rare $\mathrm{ROBO}_{4}$ variants $(1.8 \% ; \mathrm{p}=0.04)$. ROBO4 silencing or mutant ROBO4 expression in endothelial cells results in impaired barrier function and a synthetic repertoire suggestive of endothelial-to-mesenchymal transition; concordant findings are observed in patient aortic walls and Robo4-deficient animal models. 
I. Luyckx: None. A.A. Kumar: None. E. Gillis: None. R.A. Gould: None. H. Aziz: None. C.E. Woods: None. M. A. Seman-Senderos: None. G. MacCarrick: None. E. Sparks: None. A.S. MacCallion: None. L. Van Laer: None. H.C. Dietz: None. A. Verstraeten: None. B.L. Loeys: None.

\section{C10.3}

Investigating atherosclerosis progression through singlecell transcriptional profiling of immune cells of the atherosclerotic plaque

A. Sartori ${ }^{1}$, K. Thanopoulou ${ }^{2}$, C. Borel ${ }^{1}$, M. Manioudaki ${ }^{2}$, I. Galani ${ }^{2}$, E. Andreakos ${ }^{2}$, E. T. Dermitzakis ${ }^{1,3,4}$

${ }^{1}$ University of Geneva, Geneva, Switzerland, ${ }^{2}$ Biomedical Research Foundation of the Academy of Athens, Athens, Greece, ${ }^{3}$ Health 2030 Genome Center, Geneva, Switzerland, ${ }^{4}$ iGE3 Institute of Genetics and Genomics of Geneva, Geneva, Switzerland

Atherosclerosis is a chronic vascular disease characterized by the deposition of lipid molecules into the arteria intima and the formation of plaques. The pathogenesis of the plaque is a dynamic process and its cellular composition is a major determinant factor in disease development. In this study, we propose a single-cell RNA-seq approach to characterize the immune cell type composition of the plaque at different disease stages from CD45+ sorted immune cells from carotid tissue. We aim to reveal the degree of heterogeneity and variability of cell composition in plaques among individuals, its influence in plaque development, and transcriptional differences between individuals with plaque fissuring (symptomatic) vs. asymptomatic individuals. We have sequenced 16225 single-cells from 3 symptomatic and 7 asymptomatic patients (median 1152 genes; 360k reads per cell). The top highly expressed genes including MALAT1, CD74, B2M and TMSB4X were associated with atherosclerotic lesions, inflammation and adverse cardiovascular outcome. We identified 15 clusters of co-expressed genes differentiating between immune cell subtypes at the plaque level composition with a major proportion of $\mathrm{T}$ cells and macrophages, suggesting that the plaque exhibits the characteristics of a classically chronic inflamed site. We identified 4976 differentially expressed genes among clusters suggesting the presence of a high degree of cell heterogeneity in the plaque. Moreover, Gene Ontology revealed enrichment of pathways involved in pathogenesis of inflammatory diseases, apoptosis, immune response, such as NF-kB and TNF. Further work aims to investigate the presence of an expression signature between symptomatic and asymptomatic patients to better characterize disease progression.
A. Sartori: None. K. Thanopoulou: None. C. Borel: None. M. Manioudaki: None. I. Galani: None. E. Andreakos: None. E.T. Dermitzakis: None.

\section{C10.4}

Metabolomic profiling of ANGPTL3 deficiency

\section{E. Tikkanen, P. Würtz}

\section{Nightingale Health Ltd., Helsinki, Finland}

Introduction: Loss-of-function variants in the angiopoietin-like 3 gene (ANGPTL3) have been associated with low levels of plasma lipoproteins and decreased coronary artery disease risk. We aimed to determine detailed metabolic effects of genetically-induced ANGPTL3 deficiency in fasting and postprandial state.

Materials and Methods: We studied individuals carrying S17X loss-of-function mutation inANGPTL3 (6 homozygous and 32 heterozygous carriers) and 38 noncarriers. Nuclear magnetic resonance metabolomics was used to quantify 225 circulating metabolic measures. We compared metabolic differences between loss-of-function carriers and noncarriers in fasting state and after a high fat meal.

Results: In fasting, ANGPTL3 deficiency was characterized by similar extent of reductions in low-density lipoprotein cholesterol $(0.74 \mathrm{SD}$-units lower concentration per loss-of-function allele [95\%CI 0.42-1.06]) as observed for many triglyceride-rich lipoprotein measures, including very-low-density lipoprotein cholesterol (0.75 [0.45-1.05]). Within most lipoprotein subclasses, absolute levels of cholesterol were decreased more than triglycerides, resulting in the relative proportion of cholesterol being reduced within triglyceride-rich lipoproteins and their remnants. Further, beta-hydroxybutyrate was elevated $(0.55$ [0.21-0.89]). Homozygous ANGPTL3 loss-of-function carriers showed essentially no postprandial increase in triglyceride-rich lipoproteins and fatty acids, without evidence for adverse compensatory metabolic effects.

Conclusion: In addition to overall triglyceride and lowdensity lipoprotein cholesterol lowering effects, ANGPTL3 deficiency results in reduction of cholesterol proportion within triglyceride-rich lipoproteins and their remnants. Further, ANGPTL3 loss-of-function carriers had elevated ketone body production, suggesting enhanced hepatic fatty acid beta-oxidation. The detailed metabolic profile in human knockouts of ANGPTL3 reinforces inactivation of ANGPTL3 as a promising therapeutic target for decreasing cardiovascular risk.

E. Tikkanen: A. Employment (full or part-time); Significant; Nightingale Health Ltd. P. Würtz: A. Employment (full or part-time); Significant; Nightingale Health Ltd. 


\section{C10.5}

The Future is Now: Genomic Studies Must be Globally Representative

G. L. Wojcik', M. Graff ${ }^{2}$, K. Nishimura ${ }^{3}$, R. Tao ${ }^{4}$, J. Haessler ${ }^{3}$, C. R. Gignoux ${ }^{1}$, H. M. Highland ${ }^{5}$, Y. M. Patel ${ }^{6}$, S. A. Bien ${ }^{3}$, S. Buyske ${ }^{7}$, C. Haiman ${ }^{8}$, C. Kooperberg, L. Le Marchand ${ }^{10}$, R. J. F. Loos ${ }^{11}$, T. C. Matise ${ }^{7}$, U. Peters ${ }^{9}$, E. E. Kenny ${ }^{\text {I2 }}$, C. S. Carlson ${ }^{9}$, K. E. North ${ }^{2}$

${ }^{1}$ Stanford University, Stanford, CA, United States, ${ }^{2}$ Department of Epidemiology, University of North Carolina, Chapel Hill, NC, United States, ${ }^{3}$ Fred Hutchinson Cancer Research Center, Seattle, WA, United States, ${ }^{4}$ Vanderbilt University Medical Center, Nashville, TN, United States, ${ }^{5}$ University of North Carolina, Chapel Hill, NC, United States, ${ }^{6}$ Keck School of Medicine, University of Southern California, Los Angeles CA, Los Angeles, CA, United States, ${ }^{7}$ Rutgers University, New Brunswick, NJ, United States, ${ }^{8}$ Keck School of Medicine, University of Southern California, Los Angeles, CA, United States, ${ }^{9}$ Fred Hutchinson Cancer Center, Seattle, WA, United States, ${ }^{10}$ University of Hawaii, Honolulu, HI, United States, ${ }^{11}$ Icahn School of Medicine at Mount Sinai, New York NY, New York, NY, United States, ${ }^{12}$ Icahn School of Medicine at Mount Sinai, New York, NY, United States

The technological revolution in human genetics is empowering population-level investigations into the biology of complex traits, drug development, and clinical guidelines. Although these discoveries rely on genetic variation present across individuals, association studies have overwhelmingly been performed in populations of European descent.

In light of differential genetic architecture between populations, biased representation in research can exacerbate existing disease and health care disparities. Critical variants may be missed if they are at a low frequency or completely absent in European populations, especially as the field shifts its attention towards rare variants, which are more likely to be population-specific. More concerning, the effect sizes and their derived risk prediction scores derived in one population will not accurately extrapolate to other populations.

Herein, we describe our recent work of the Population Architecture using Genomics and Epidemiology (PAGE) study, where we have conducted a GWAS of 26 cardiometabolic phenotypes in 49,839 non-European individuals. We identify 27 novel loci and 38 secondary signals at known cardiometabolic loci, as well as replicate 1,444 GWAS catalog associations across these traits. Our data shows strong evidence of effect-size heterogeneity across ancestries for published GWAS associations, substantial benefits for fine mapping using diverse cohorts, and insights into clinical implications. We fear that the lack of representation of diverse populations in genetic research will result in inequitable access to precision medicine for those with the highest burden of disease. Thus, we strongly advocate for continued, large genome-wide efforts in diverse populations to maximize genetic discovery and reduce health disparities.

G.L. Wojcik: None. M. Graff: None. K. Nishimura: None. R. Tao: None. J. Haessler: None. C.R. Gignoux: None. H.M. Highland: None. Y.M. Patel: None. S.A. Bien: None. S. Buyske: None. C. Haiman: None. C. Kooperberg: None. L. Le Marchand: None. R.J.F. Loos: None. T.C. Matise: None. U. Peters: None. E.E. Kenny: None. C.S. Carlson: None. K.E. North: None.

\section{C10.6}

Genetics of human plasmalipidome and its link to cardiovascular diseases

R. Tabassum ${ }^{I}$, J. T. Rämö ${ }^{I}$, P. Ripatti ${ }^{1}$, J. T. Koskela ${ }^{I}$, M. Kurki ${ }^{1,2,3}$, J. Karjalainen ${ }^{1,4,5}$, S. Hassan ${ }^{1}$, J. NunezFontarnau $^{l}$, T. T. Kiiskinen ${ }^{1}$, S. Soderlund ${ }^{6}$, N. Matikainen ${ }^{6,7}$, M. J. Gerl ${ }^{8}$, M. A. Surma ${ }^{8,9}, C . K_{\text {Llose }}^{8}$, N. O. Stitziel ${ }^{10,11,12}$, H. Laivuori ${ }^{1,13,14}$, A. S. Havulinna ${ }^{1,15}$, S. K. Service ${ }^{16}$, V. Salomaa ${ }^{15}$, M. Pirinen ${ }^{1,17,18}$, F. Project ${ }^{1}$, M. Jauhiainen ${ }^{15,19}$, M. J. Daly ${ }^{1,4}$, N. B. Freimer ${ }^{16}$, A. Palotie ${ }^{1,4,20}$, M. Taskinen ${ }^{6}$, K. Simons ${ }^{8,21}$, S. Ripatti ${ }^{1,4,22}$

${ }^{l}$ Institute for Molecular Medicine Finland, HiLIFE, University of Helsinki, Helsinki, Helsinki, Finland, ${ }^{2}$ Broad Institute of MIT and Harvard, Cambridge, MA, United States, ${ }^{3}$ Psychiatric \& Neurodevelopmental Genetics Unit, Massachusetts General Hospital, Boston, MA, United States, ${ }^{4}$ Broad Institute of the Massachusetts Institute of Technology and Harvard University, Cambridge, MA, United States, ${ }^{5}$ Analytic and Translational Genetics Unit, Massachusetts General Hospital and Harvard Medical School, Boston, MA, United States, ${ }^{6}$ Research Programs Unit, Diabetes \& Obesity, University of Helsinki and Department of Internal Medicine, Helsinki University Hospital, Helsinki, Finland, ${ }^{7}$ Endocrinology, Abdominal Center, Helsinki University Hospital, Helsinki, Finland, ${ }^{8}$ Lipotype GmbH, Dresden, Germany, ${ }^{9}$ PORT, Wroclaw, Poland, ${ }^{10}$ Cardiovascular Division, Department of Medicine, Washington University School of Medicine, Saint Louis, MO, United States, ${ }^{11}$ Department of Genetics, Washington University School of Medicine, Saint Louis, MO, United States, ${ }^{12}$ McDonnell Genome Institute, Washington University School of Medicine, Saint Louis, MO, United States, ${ }^{13}$ Department of Obstetrics and Gynecology, Tampere University Hospital and University 
of Tampere, Helsinki, Finland, ${ }^{14}$ Medical and Clinical Genetics, University of Helsinki and Helsinki University Hospital, Helsinki, Finland, ${ }^{15}$ National Institute for Health and Welfare, Helsinki, Finland, ${ }^{16}$ Center for Neurobehavioral Genetics, Semel Institute for Neuroscience and Human Behavior, University of California, Los Angeles, CA, United States, ${ }^{17}$ Department of Public Health, University of Helsinki, Helsinki, Finland, ${ }^{18}$ Helsinki Institute for Information Technology HIIT and Department of Mathematics and Statistics, University of Helsinki, Helsinki, Finland, ${ }^{19}$ Minerva Foundation Institute for Medical Research, Biomedicum, Helsinki, Finland, ${ }^{20}$ Psychiatric \& Neurodevelopmental Genetics Unit, Department of Psychiatry, Analytic and Translational Genetics Unit, Department of Medicine, and the Department of Neurology, Massachusetts General Hospital, Boston, MA, United States, ${ }^{21}$ Max Planck Institute of Cell Biology and Genetics, Dresden, Germany, ${ }^{22}$ Department of Public Health, Clinicum, Faculty of Medicine, University of Helsinki, Helsinki, Finland

Introduction: Human plasma comprises of numerous molecular lipid species that outperform traditional risk factors in cardiovascular diseases (CVD) risk prediction.

Methods: We performed a genome-wide association study (GWAS) of 141 lipid species in 2,181 individuals, followed by phenome-wide scans (PheWAS) with $23 \mathrm{CVD}$ end-points in $>500,000$ individuals from the Finnish and UK Biobanks. We also determined (1) how heritable are lipid species and do they share genetic components and (2) could we gain mechanistic insights into lipid metabolism.

Results: SNP based heritabilities for lipid species ranged from $10-54 \%$. Long polyunsaturated lipids showed highest heritability and genetic sharing, suggesting considerable genetic regulation at fatty acids levels. The observed low genetic sharing between traditional lipids and lipid species suggested that routine lipid screens may fail to capture potential disease risk factors. GWAS identified 35 lipidspecies-associated loci $\left(\mathrm{P}<5 \times 10^{-8}\right)$, with 15 new lipid loci and 37 new SNP-lipid species pair associations e.g. association between $A B C G 5 / 8$ and $\operatorname{CE}(20: 2 ; 0)$. We show that $L P L$ (lipoprotein lipase) may have more efficient hydrolysis of medium length TAGs (triacylglycerides) than other TAGs. The association patterns of SYNGR1, MIR100HG, and PTPRN2 suggested their probable roles in desaturation and/or elongation of fatty acids. PheWAS revealed that 7 of 35 lipid-species-associated loci also associate with CVD related outcomes, including $A B C G 5 / 8, A P O A 1, B L K$, $F A D S 2$, $L P L$, and two new loci-COL5Aland GALNT16 (false discovery rate $<0.05$ ).

Conclusion: We demonstrate that lipidomics enable deeper insights to the genetic regulation of lipid metabolism than clinically used lipid measures, which in turn might help guide future disease biomarker discovery.

R. Tabassum: None. J.T. Rämö: None. P. Ripatti: None. J.T. Koskela: None. M. Kurki: None. J. Karjalainen: None. S. Hassan: None. J. Nunez-Fontarnau: None. T.T. Kiiskinen: None. S. Soderlund: None. N. Matikainen: None. M.J. Gerl: A. Employment (full or part-time); Significant; Lipotype GmbH. M.A. Surma: A. Employment (full or part-time); Significant; PORT. E. Ownership Interest (stock, stock options, patent or other intellectual property); Significant; Lipotype GmbH. C. Klose: A. Employment (full or part-time); Significant; Lipotype GmbH. N.O. Stitziel: None. H. Laivuori: None. A.S. Havulinna: None. S.K. Service: None. V. Salomaa: None. M. Pirinen: None. F. Project: None. M. Jauhiainen: None. M.J. Daly: None. N.B. Freimer: None. A. Palotie: None. M. Taskinen: None. K. Simons: E. Ownership Interest (stock, stock options, patent or other intellectual property); Significant; Lipotype GmbH. S. Ripatti: None.

\section{C11 Statistical and population genetics}

\section{C11.1}

Maximum likelihood method quantifies the overall contribution of gene-environment interaction to complex traits: an application to obesity traits
J. Sulc ${ }^{1}$, N. Mounier ${ }^{1}$, T. Winkler ${ }^{2}$, A. Wood $^{3}$, T. Frayling, I. M. Heid ${ }^{2}$, M. R. Robinson ${ }^{4}$, Z. Kutalik ${ }^{1,3}$
${ }^{1}$ University Center for Primary Case and Public Health, Lausanne, Switzerland, ${ }^{2}$ Department of Genetic Epidemiol- ogy, University of Regensburg, Regensburg, Germany, ${ }^{3}$ Genetics of Complex Traits, University of Exeter Medical School, University of Exeter, Exeter, United Kingdom, ${ }^{4}$ Department of Computational Biology, University of Lausanne, Lausanne, Switzerland

Introduction: As genome-wide association studies (GWAS) increased in size, numerous gene-environment interactions (GxE) have been discovered, many of which however explore only one environment at a time and may suffer from statistical artefacts leading to biased interaction estimates.

Materials and Methods: Here we propose a maximum likelihood method to estimate the contribution of GxE to complex traits taking into account all interacting environmental variables at the same time, without the need to measure any. This is possible because GxE induces fluctuations in the conditional trait variability, the extent of which depends on the strength of GxE. The approach can be 
applied to continuous outcomes and for single SNPs or genetic risk scores (GRS).

Results: Extensive simulations covering a wide range of scenarios demonstrated that our method yields unbiased interaction estimates. We also offer a strategy to distinguish specific GxE from general heteroscedasticity (scale effects). Applying our method to 32 obesity-related traits in the UK Biobank reveals that for body mass index (BMI) the GRSxE explains an additional $1.9 \%$ variance on top of the $5.2 \%$ GRS contribution. However, this interaction is not specific to the BMI-GRS and holds for any variable equally correlated with BMI. On the contrary, the GRSxE interaction effect for leg impedance $(0.07)$ is significantly $\left(\mathrm{P}<10^{-56}\right)$ larger than it would be expected for a similarly correlated variable $(-0.16)$.

Conclusion: We showed that our method could robustly detect the global contribution of GxE to complex traits, which turned out to be substantial for some obesity measures.

J. Sulc: None. N. Mounier: None. T. Winkler: None. A. Wood: None. T. Frayling: None. I.M. Heid: None. M.R. Robinson: None. Z. Kutalik: None.

\section{C11.2}

\section{Leveraging correlated risks to increase power in Genome-Wide Association Studies}

\author{
N. Mounier ${ }^{1,2}$, P. R. H. J. Timmers ${ }^{3}$, J. F. Wilson ${ }^{3,4}$, \\ P. K. Joshi, Z. Kutalik ${ }^{1,2}$
}

${ }^{1}$ University Center for Primary Care and Public Health, Lausanne, Switzerland, ${ }^{2}$ Swiss Institute of Bioinformatics, Lausanne, Switzerland, ${ }^{3}$ Centre for Global Health Research, Usher Institute of Population Health Sciences and Informatics, Edinburgh, United Kingdom, ${ }^{4} \mathrm{MRC}$ Human Genetics Unit, Institute of Genetics and Molecular Medicine, Edinburgh, United Kingdom

Genome-Wide Association Studies (GWASs) are nowadays often conducted in $>1 \mathrm{M}$ samples. Improved discovery, by further increasing study sizes is not the only strategy. Leveraging published studies of related traits can improve inference. To this end, we developed a Bayesian GWAS approach that builds informative priors from GWASs of related risk factors. Mendelian Randomisation is used to derive multivariate causal effects of a set of iteratively selected risk factors on the target outcome. These causal effects of the risk factors on the outcome are then combined with the SNP's effect on the identified risk factors to build a Bayesian prior for the SNP's effects on the outcome. We found a set of universally optimal parameters (instrument strength, clumping stringency and shrinkage intensity) that maximizes the out-of-sample squared correlation between prior and observed effects (up to $\sim 40 \%$ ). We use Bayes Factors (BFs) to quantify the evidence in favour of the prior and derived an analytical formula for the null BFs distribution, which led to a 6-fold reduced runtime and a more accurate estimation of P-values, compared to permutation-based approaches. We are able to gauge genetic risk score (GRS) performance using only summary statistics from training and test samples. Its application revealed that GRSs based on posterior effects outperform standard GRS. Applying this technique to human lifespan, we identified 7 new loci $(P<5 e-8)$, which were missed by standard GWAS. The method and visualization of its results have been implemented in an $\mathrm{R}$ package (https://github. com/n-mounier/bGWAS).

N. Mounier: None. P.R.H.J. Timmers: None. J.F. Wilson: None. P.K. Joshi: None. Z. Kutalik: None.

C11.3

One and a half million genome wide-association studies of brain morphometry: a proof-of-concept study

G. Roshchupkin ${ }^{1}$, M. A. Ikram ${ }^{1}$, K. Wittfeld ${ }^{2}$, M. Zwiers ${ }^{3}$, N. Jahanshad ${ }^{4}$, A. Teumer ${ }^{2}$, P. Thompson ${ }^{4}$, B. Franke ${ }^{3}$, H. Grabe ${ }^{2}$, W. Niessen ${ }^{1}$, H. H. H. Adams ${ }^{1}$

${ }^{1}$ Erasmus MC, Rotterdam, Netherlands, ${ }^{2}$ University of Greifswald, Greifswald, Germany, ${ }^{3}$ Radboudumc, Nijmegen, Netherlands, ${ }^{4}$ University of Southern California, Los Angeles, CA, United States

Objective: The human brain has an intricate structure that is partly determined by genetics. While neuroimaging can discern millions of morphometric features, genome-wide association studies have been limited to only a number of aggregate measures due to technical reasons.

Methods: We studied 18.000 individuals from 12 cohorts within the CHARGE consortium and UK Biobank participants. The structure of the cortical and subcortical grey matter was characterized using voxel-based morphometry, a technique that generates a volume for each of the 1.5 million voxels. Subsequently, genome-wide association studies were performed for all these voxels using a newly developed software algorithm. The genome-wide brain-wide significance threshold was determined at $\mathrm{p}=5 \times 10^{-13}$.

Results: The association analyses between 9 million genetic variants and 1.5 million brain voxels took $17 \mathrm{~h}$ to compute, which would require over 2 years using conventional software. We found 1.270.907 genome-wide brainwide significant associations between genetic variants and grey matter voxels, distributed between 55 brain regions with minimum $\mathrm{p}$-value $=2 \times 10^{-87}$. These associations came from 930 variants that mapped to 29 independent loci. 
We found 62.920 unique voxels significantly associated at least with one variant.

Conclusion: The genome-wide interrogation of highresolution neuroimaging data is a powerful approach to identify genetic determinants of brain structure, even at sample sizes considerably smaller than studies of aggregate measures. This successful first attempt at high-throughput GWAS could be extended to various fine-grained (endo) phenotypes, which might prove useful for understanding the genetic architecture of other complex traits.

G. Roshchupkin: None. M.A. Ikram: None. K. Wittfeld: None. M. Zwiers: None. N. Jahanshad: None. A. Teumer: None. P. Thompson: None. B. Franke: None. H. Grabe: None. W. Niessen: None. H.H.H. Adams: None.

\section{C11.4}

Genome-wide copy number variant association study reveals several novel disease-associated loci

\section{Lepamets $^{1,2}$, K. Lepik ${ }^{3,1}$, Z. Kutalik ${ }^{4}$, R. Mägi ${ }^{1}$}

${ }^{1}$ Estonian Genome Center, Institute of Genomics, University of Tartu, Tartu, Estonia, ${ }^{2}$ Institute of Molecular and Cell Biology, University of Tartu, Tartu, Estonia, ${ }^{3}$ Institute of Computer Science, University of Tartu, Tartu, Estonia, ${ }^{4}$ Institute of Social and Preventive Medicine, Lausanne University Hospital, Lausanne, Switzerland

Genome-wide association study (GWAS) is a successful tool for identifying common single nucleotide polymorphisms (SNPs) contributing to complex human traits and diseases. However, relatively little of heritability of those traits has been explained by SNPs due to their small effect sizes. Therefore, it is necessary to analyze other types of genetic variations including DNA copy number variations (CNVs).

Even though large (>100kbp) rare CNVs are studied for a wide range of complex traits and diseases, detecting shorter CNVs from SNP genotyping array data remains challenging due to large number of false positives. To address this issue, we have developed a statistical model to predict the quality of possible CNV regions detected by PennCNV software. Our model outperforms the best currently published $\mathrm{CNV}$ quality score.

Using our CNV quality measure, we conducted genomewide $\mathrm{CNV}$ analysis on ten complex diseases (incl. type 2 diabetes, inflammatory bowel disease (IBD) and rheumatoid arthritis (RA), etc.) in up to 398,662 unrelated white British individuals from UK Biobank cohort. In total, we found 18 genome-wide significant $(\mathrm{P}<1.7 * 10 \mathrm{e}-6) \quad \mathrm{CNV}$-disease associations, out of which 15 are novel. Noteworthy examples are association between RA and a 6p21.3 deletion $\left(\mathrm{P}=8.5^{*} 10 \mathrm{e}-7\right)$ overlapping a known strong GWAS signal
$(\mathrm{P}=10 \mathrm{e}-250)$ and a 10p11.21 deletion associated with IBD $\left(\mathrm{P}=9.1^{*} 10 \mathrm{e}-7\right)$ that overlaps the tight junction-related gene PARD3, which has been associated with IBD in candidate gene studies. Genome-wide CNV association studies help us to better understand/fine-map the underlying genetic mechanisms for complex diseases. Our analyses are a first step in compiling a large CNV-trait database.

M. Lepamets: None. K. Lepik: None. Z. Kutalik: None. R. Mägi: None.

\section{C11.5}

Fine-scale population structure and demographic change through time and space in the Netherlands

\section{R. P. Byrne ${ }^{1}$, W. van Rheenen ${ }^{2}$, L. H. van den Berg',} J. H. Veldink ${ }^{2}$, R. L. McLaughlin ${ }^{1}$

${ }^{1}$ Smurfit institute of genetics, Trinity College Dublin, Dublin, Ireland, ${ }^{2}$ Department of Neurology, Brain Centre Rudolf Magnus, University Medical Center Utrecht, Utrecht, Netherlands

Introduction: We present a detailed genetic analysis of population structure, migration and demographic changes in the Netherlands using powerful methods incorporating haplotype sharing and local geographic origin.

Methods: We combined genome-wide SNP and geographic origin data $(\mathrm{N}=1422)$ to explore the interplay between genetics and geography in the Netherlands. Using ChromoPainter/fineSTRUCTURE we partitioned our data based on patterns of haplotype sharing to identify fine-scale population structure. We analysed patterns of identity-bydescent (IBD) sharing for segments of different lengths to estimate the time depth of the structure. We investigated whether the structure reflects barriers to gene flow using the Estimated Effective Migration Surfaces (EEMS) method. We also analysed spatial clines in haplotype sharing from neighbouring countries using a European reference $(\mathrm{N}=$ 4514). Finally, we estimated recent changes in effective population size using the IBDNe method.

Results: We identified 16 genetic clusters which correlate closely with regional geography. At its finest level, clustering distinguishes subtly different eastern and western genetic groups in the North-Brabant province, while deeper clustering delineates a north-south split bisecting the country. We estimate that this north-south genetic structure is at least 2700 years old, while east-west structure is more recent. EEMs revealed a strong migrational cold spot splitting the country north to south, overlapping the Rhine. Overlaying this deep structure we observe strong opposing northeast-southwest spatial clines in German and Belgian haplotype sharing. Finally we infer a super-exponential population growth in the past 50 generations. 
Grants: MND Association (957-799); Science Foundation Ireland (17/CDA/4737)

R.P. Byrne: None. W. van Rheenen: None. L.H. van den Berg: None. J.H. Veldink: None. R.L. McLaughlin: None.

\section{C11.6}

The landscape of pervasive horizontal pleiotropy in human genetic variation is driven by extreme polygenicity of human traits and diseases

\section{Verbanck, D. M. Jordan, R. Do}

The Charles Bronfman Institute for Personalized Medicine, Icahn School of Medicine at Mount Sinai, New York, NY, United States

Understanding the nature of horizontal pleiotropy, where one genetic variant has independent effects on multiple observable traits, is vitally important for our understanding of the genetic architecture of human phenotypes. Many recent studies have pointed to the existence of horizontal pleiotropy, but its extent remains unknown, largely due to difficulty in disentangling the inherently correlated nature of observable traits. We have developed a statistical framework to quantify horizontal pleiotropy in human genetic variation, using a two-component score computed from summary statistics. This score uses a whitening procedure to remove correlations between observable traits and normalize effect sizes across all traits. When applied to simulated association summary statistics, our score successfully detects horizontal pleiotropy under a range of different models. When applied to 372 heritable phenotypes from the UK Biobank, our pleiotropy score detects a significant excess of horizontal pleiotropy which is pervasive throughout the human genome and especially prominent among highly polygenic phenotypes. We identify 24,968 variants in 7,831 loci with extreme horizontal pleiotropy, a majority of which have never been reported in any published GWAS. Examining functional annotations, the pleiotropy score is significantly higher in active regions and lower in inactive regions. In addition, the pleiotropy score correlates with transcriptional measures of pleiotropy, it is significantly higher for variants which are eQTLs i) for many genes across many tissues; ii) for genes whose orthologs are associated with multiple phenotypes in mice or yeast. Our results highlight the central role horizontal pleiotropy plays in the genetic architecture of human phenotypes.

M. Verbanck: None. D.M. Jordan: None. R. Do: B. Research Grant (principal investigator, collaborator or consultant and pending grants as well as grants already received); Significant; AstraZeneca, Goldfinch Bio.
C12 Intellectual Disability

\section{C12.1}

Phenotypic spectrum of novel intellectual disability syndrome due to de novo variants in $K M T 2 E$

\author{
A. O'Donnell-Luria ${ }^{1,2,3}$, L. S. Pais ${ }^{1,2}$, V. Faundes ${ }^{4,5}$, \\ KMT2E Consortium, X. Soto ${ }^{6}$, N. Papalopulu ${ }^{6}$, \\ S. Banka ${ }^{5,7}$, L. H. Rodan ${ }^{1,3}$
}

${ }^{1}$ Boston Children's Hospital, Boston, MA, United States, ${ }^{2}$ Broad Institute of MIT and Harvard, Cambridge, MA, United States, ${ }^{3}$ Harvard Medical School, Boston, MA, United States, ${ }^{4}$ Instituto de Nutrición y Tecnología de los Alimentos (INTA), Universidad de Chile, Santiago, Chile, ${ }^{5}$ Manchester Centre for Genomic Medicine, Manchester University NHS Foundation Trust, Manchester, United Kingdom, ${ }^{6}$ Division of Developmental Biology \& Medicine, The University of Manchester, Manchester, United Kingdom, ${ }^{7}$ School of Medical Sciences, The University of Manchester, Manchester, United Kingdom

$K M T 2 E$ encodes a histone methyltransferase epigenetic protein, a transcriptional regulator reported to play key roles in diverse biological processes, including cell cycle progression, and genomic stability maintenance. Here we describe the neurodevelopmental phenotype that results from disruption of KMT2E in 38 affected individuals from 36 families. From the Genome Aggregation Database (gnomAD), KMT2E is a candidate haploinsufficient gene with a LOEUF score of 0.06 . This case series includes 28 protein truncating variants (PTVs), four missense variants, and four large deletions encompassing KMT2E, most of which were confirmed to be de novo. Most affected individuals with protein truncating variants presented with mild intellectual disability, autism and/or epilepsy. Epilepsy more common in females and autism more common in males; many had relative macrocephaly and hypotonia. There appears to be a subtle facial gestalt characterized by dolichocephaly, deep set eyes with down-slanting palpebral fissures, and infraorbital creases. Individuals with microdeletions encompassing KMT2E showed a greater degree of developmental delay. Individuals with missense variants in KMT2E presented with the most severe developmental delays and treatment-resistant infantile epileptic encephalopathy; microcephaly was common in this group. Haploinsufficiency versus gain-of-function or dominant negative effects specific to these missense variants in KMT2E may explain this divergence in phenotype but requires independent validation. To support our findings for loss of function in $K M T 2 E$, we performed functional analysis by generating a kmt2e knockout Zebrafish using CRISPR-Cas9 technology. The G0 fish revealed dose-dependent phenotypes that 
were consistent with the human syndrome further proving the importance of this gene in development.

A. O'Donnell-Luria: None. L.S. Pais: None. V. Faundes: None. X. Soto: None. N. Papalopulu: None. S. Banka: None. L.H. Rodan: None.

\section{C12.2}

$C T C F$ variants in 31 individuals with a variable neurodevelopmental disorder broaden the mutational and clinical spectrum

E. D. H. Konrad ${ }^{I}$, N. Nardini ${ }^{I}$, M. Blyth ${ }^{2}$, K. Prescott ${ }^{2}$, A. M. Bouman ${ }^{3}$, E. H. Brilstra ${ }^{4}$, A. Caliebe , R. Ibitoye $^{6}$,

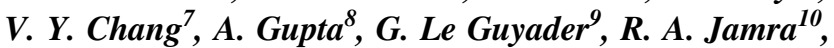
K. Platzer ${ }^{10}$, M. C. J. Jongmans ${ }^{11}$, A. Kenney ${ }^{12}$, M. Kempers ${ }^{11}$, R. Pfundt ${ }^{11}$, D. Khattar ${ }^{13}$, O. Kuismin ${ }^{14}$, E. Legius ${ }^{15}$, K. D. Lichtenbelt ${ }^{16}$, T. J. Maarup ${ }^{17}$, M. McEntagart ${ }^{18}$, K. Õunap ${ }^{19}$, M. E. Pierpont ${ }^{20}$, S. L. Santoro ${ }^{21}$, H. M. Schnelle ${ }^{22}$, E. Fassi ${ }^{23}$, D. Young ${ }^{24}$, A. Ziegler ${ }^{25}$, Deciphering Developmental Disorders (DDD) study, A. Gregor ${ }^{1}$, H. Van Esch ${ }^{15}$, C. Zweier ${ }^{1}$

${ }^{1}$ Institute of Human Genetics, Erlangen, Germany, ${ }^{2}$ Yorkshire Regional Genetics Service, Chapel Allerton Hospital, Leeds Teaching Hospitals NHS Trust, Leeds, United Kingdom, ${ }^{3}$ Department of Clinical Genetics, Erasmus Medical Center, Rotterdam, Netherlands, ${ }^{4}$ Department of Medical Genetics, University Medical Center Utrecht, Utrecht University, Utrecht, Netherlands, ${ }^{5}$ Institute for Human Genetics, Universitätsklinikum Schleswig Holstein Campus Kiel, Kiel, Germany, ${ }^{6}$ North West Thames Regional Genetics Service, Northwick Park Hospital, Harrow, United Kingdom, ${ }^{7}$ Division of Pediatric Hematology-Oncology, Department of Pediatrics, David Geffen School of Medicine, Los Angeles, CA, United States, ${ }^{8}$ Center for Individualized Medicine, Mayo Clinic, Rochester, MN, United States, ${ }^{9}$ Service de Génétique Clinique, CHU de Poitiers, Poitiers, France, ${ }^{10}$ Institute of Human Genetics, University of Leipzig Medical Center, Leipzig, Germany, ${ }^{11}$ Department of Human Genetics, Radboud University Medical Center and Radboud Institute for Molecular Life Sciences, Nijmegen, Netherlands, ${ }^{12}$ Division of Medical Genetics and Metabolism, Children's Hospital of the King's Daughters, Norfolk, VA, United States,

${ }^{13}$ Division of Human Genetics, Cincinnati Children's Hospital Medical Center, Cincinnati, OH, United States, ${ }^{14}$ Department of Clinical Genetics, Oulu University Hospital, Oulu, Finland, ${ }^{15}$ Center for Human Genetics, University Hospital Leuven, KU Leuven, Leuven, Belgium, ${ }^{16}$ Department of Genetics, University Medical Center Utrecht, Utrecht, Netherlands, ${ }^{17}$ Department of Genetics, Kaiser Permanente, Los Angeles, CA, United States, ${ }^{18}$ South West Thames Regional Genetics Centre, St.
George's Healthcare NHS Trust, St. George's, University of London, London, United Kingdom, ${ }^{19}$ Department of Clinical Genetics, United Laboratories, Tartu University Hospital, Tartu, Estonia, ${ }^{20}$ Department of Pediatrics, Division of Genetics and Metabolism, University of Minnesota, Minneapolis, MN, United States, ${ }^{21}$ Division of Molecular and Human Genetics, Columbus, OH, United States, ${ }^{22}$ Department of Medical Genetics, Haukeland University Hospital, Bergen, Norway, ${ }^{23}$ Division of Genetics and Genomic Medicine, Department of Pediatrics, Washington University School of Medicine, St. Louis, MO, United States, ${ }^{24}$ Adult Metabolic Diseases Clinic, Vancouver General Hospital, Vancouver, BC, Canada, ${ }^{25}$ Service de Génétique, CHU d'Angers, Angers, France

Background: Pathogenic variants within the chromatin organizer $C T C F$ were identified as causative for a variable neurodevelopmental disorder (NDD) in four individuals, so far.

Methods: Through an international collaboration we collected molecular and clinical data from 31 individuals with pathogenic variants in $C T C F$. We performed transcriptome analysis on RNA extracted from patient blood samples and utilized Drosophila melanogaster to investigate the impact of $C t c f$ dosage alteration on nervous system development and function.

Results: The variants identified in $C T C F$ included two large deletions, seven truncating, two splice site and 16 different missense variants, one of them in a mosaic state. One case was familial. The associated phenotype was very variable and ranged from mild developmental delay and normal IQ to severe intellectual disability. Feeding difficulties, failure to thrive $(67 \%)$ and behavioral $(76 \%)$ and vision anomalies (57\%) were common. Variable other features included microcephaly (38\%), short stature (21\%), and cardiac defects (29\%). RNA-Seq in five individuals identified 3,828 deregulated genes enriched for known NDD genes and biological processes such as transcriptional regulation. Ctcf knockdown and overexpression in Drosophila showed unaltered morphology of neuromuscular synapses and multiple dendrite neurons but resulted in impaired gross neurological functioning in the climbing assay and learning and memory deficits in the courtship conditioning paradigm.

Conclusion: We broaden the mutational and clinical spectrum of CTCF-associated NDDs and gain insights into the functional role of CTCF by identifying deregulated genes and showing that Ctcf dosage alterations result in nervous system defects in Drosophila, modeling important aspects of the human condition.

E.D.H. Konrad: None. N. Nardini: None. M. Blyth: None. K. Prescott: None. A.M. Bouman: None. E.H. Brilstra: None. A. Caliebe: None. R. Ibitoye: None. V.Y. 
Chang: None. A. Gupta: None. G. Le Guyader: None. R. A. Jamra: None. K. Platzer: None. M.C.J. Jongmans: None. A. Kenney: None. M. Kempers: None. R. Pfundt: None. D. Khattar: None. O. Kuismin: None. E. Legius: None. K.D. Lichtenbelt: None. T.J. Maarup: None. M. McEntagart: None. K. Õunap: None. M.E. Pierpont: None. S.L. Santoro: None. H.M. Schnelle: None. E. Fassi: None. D. Young: None. A. Ziegler: None. A. Gregor: None. H. Van Esch: None. C. Zweier: None.

\section{C12.3}

De novo variants disturbing the transactivation capacity of POU3F3 cause a characteristic neurodevelopmental disorder

L. Snijders Blok ${ }^{1,2}$, T. Kleefstra ${ }^{1}$, H. Venselaar ${ }^{1}$, S. Maas ${ }^{3}$, H. Y. Kroes ${ }^{4}$, A. M. A. Lachmeijer ${ }^{4}$, K. L. I. van Gassen ${ }^{4}$,

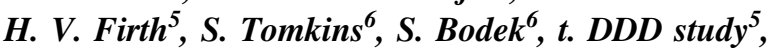

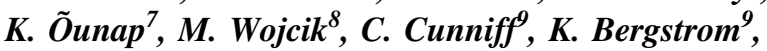
Z. Powis ${ }^{10}$, S. Tang ${ }^{10}$, D. N. Shinde ${ }^{10}, C . A u^{11}$,

A. D. Iglesias ${ }^{11}$, K. Izumi ${ }^{12}$, J. Leonard ${ }^{12}$, A. A. Tayyoun ${ }^{12}$, S. W. Baker ${ }^{12}$, M. Tartaglia ${ }^{13}$, M. Niceta ${ }^{13}$, M. L. Dentici ${ }^{13}$, N. Okamoto $^{14}$, N. Miyake ${ }^{15}$, N. Matsumoto ${ }^{15}$, A. Vitobello ${ }^{16}$, L. Faivre ${ }^{16}$, C. Philippe ${ }^{16}$, C. Gilissen ${ }^{1}$, L. van de Wiel ${ }^{1}$, R. Pfundt ${ }^{1}$, P. Deriziotis ${ }^{2}$, H. G. Brunner ${ }^{1,17}$, S. E. Fisher ${ }^{2}$

${ }^{1}$ Radboud University Medical Center, Nijmegen, Netherlands, ${ }^{2}$ Max Planck Institute for Psycholinguistics, Nijmegen, Netherlands, ${ }^{3}$ Amsterdam UMC, Amsterdam, Netherlands, ${ }^{4}$ University Medical Center Utrecht, Utrecht, Netherlands, ${ }^{5}$ Wellcome Trust Sanger Institute, Hinxton, United Kingdom, ${ }^{6}$ University Hospitals Bristol NHS Foundation Trust, Bristol, United Kingdom, ${ }^{7}$ Tartu University Hospital and Institute of Clinical Medicine, Tartu, Estonia, ${ }^{8}$ the Broad Institute of MIT and Harvard, Cambridge, MA, United States, ${ }^{9}$ Weill Cornell Medicine, New York, NY, United States, ${ }^{10}$ Ambry Genetics, Aliso Viejo, CA, United States, ${ }^{11}$ New York Presbyterian Hospital, New York, NY, United States, ${ }^{12}$ the Children's Hospital of Philadelphia, Philadelphia, PA, United States, ${ }^{13}$ Bambino Gesù Children Hospital, Rome, Italy, ${ }^{14}$ Osaka Women's and Children's Hospital, Izumi, Japan, ${ }^{15}$ Yokohama City University Graduate School of Medicine, Yokohama, Japan, ${ }^{16} \mathrm{CHU}$ Dijon Bourgogne, Dijon, France, ${ }^{17}$ Maastricht University Medical Center, Maastricht, Netherlands

The POU3F3 protein, also referred to as Brain-1, is a wellknown transcription factor involved in development of the central nervous system, but it has not previously been associated with a neurodevelopmental disorder. Here, we report the identification and phenotypic characterization of nineteen individuals with heterozygous disruptions of
POU3F3, most of which are de novo variants. All individuals had developmental delays and/or intellectual disability. Impairments in speech and language skills were common, along with characteristic prominent and cupped ears. $P O U 3 F 3$ is an intronless gene, insensitive to nonsense-mediated decay, and thirteen individuals carried variants that were predicted to yield a truncated protein. All truncating variants that we tested in cellular models led to aberrant subcellular localization of the encoded protein. Luciferase assays demonstrated negative effects of these alleles on transcriptional activation of a reporter with a FOXP2-derived binding motif, also bound by POU3F2. In addition to the loss-of-function variants, five individuals had missense variants that clustered at specific positions within the functional domains, and one small in-framedeletion was identified. Some missense variants showed reduced transactivation capacity in our assays, and one variant displayed gain-of-function effects, suggesting a distinct pathophysiological mechanism. In Bioluminescence Resonance Energy Transfer (BRET) interaction assays, all the truncated versions of POU3F3 that we tested had significantly impaired dimerization capacities, while all missense variants showed unaffected dimerization with wild-type POU3F3. Taken together, our identification and functional cell-based analyses of pathogenic variants in $P O U 3 F 3$, coupled with in-depth clinical characterization, implicate disruptions of this gene in a potentially recognizable neurodevelopmental disorder.

L. Snijders Blok: None. T. Kleefstra: None. H. Venselaar: None. S. Maas: None. H.Y. Kroes: None. A.M.A. Lachmeijer: None. K.L.I. van Gassen: None. H.V. Firth: None. S. Tomkins: None. S. Bodek: None. T. DDD study: None. K. Õunap: None. M. Wojcik: None. C. Cunniff: None. K. Bergstrom: None. Z. Powis: None. S. Tang: None. D.N. Shinde: None. C. Au: None. A.D. Iglesias: None. K. Izumi: None. J. Leonard: None. A.A. Tayyoun: None. S.W. Baker: None. M. Tartaglia: None. M. Niceta: None. M.L. Dentici: None. N. Okamoto: None. N. Miyake: None. N. Matsumoto: None. A. Vitobello: None. L. Faivre: None. C. Philippe: None. C. Gilissen: None. L. van de Wiel: None. R. Pfundt: None. P. Deriziotis: None. H.G. Brunner: None. S.E. Fisher: None.

\section{C12.4}

De novo variants in MAPK8IP3 cause intellectual disability with variable brain anomalies
K. Platzer ${ }^{1}$, H. Sticht ${ }^{2}$, S. L. Edwards ${ }^{3}$,W. Allen ${ }^{4}$, K. M. Angione ${ }^{5}$, M. T. Bonati ${ }^{6}$, C. Brasington ${ }^{7}$, M. T. Cho $^{8}$, L. A. Demmer ${ }^{7}$, T. Falik-Zaccai ${ }^{9,10}$, C. N. Gamble ${ }^{11}$, Y. Hellenbroich ${ }^{12}$, M. Iascone ${ }^{13}$, F. Kok ${ }^{14}$, S. Mahida ${ }^{15}$, H. Mandel ${ }^{9}$, T. Marquardt ${ }^{16}$,

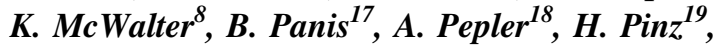


L. Ramos ${ }^{14}$, D. N. Shinde ${ }^{20}$, C. Smith-Hicks ${ }^{15}$, A. P. A. Stegmann $^{21}$, P. Stöbe ${ }^{18}$, C. T. R. M. Stumpel ${ }^{21}$, C. Wilson ${ }^{4}$, J. R. Lemke ${ }^{1}$, N. Di Donato ${ }^{22}$, K. G. Miller ${ }^{3}$, R. Abou $J^{J a m r a}{ }^{l}$

${ }^{1}$ Institute of Human Genetics, University of Leipzig Medical Center, Leipzig, Germany, ${ }^{2}$ Institute of Biochemistry, EmilFischer Center, Friedrich-Alexander-Universität ErlangenNürnberg, Erlangen, Germany, ${ }^{3}$ Genetic Models of Disease Laboratory, Oklahoma Medical Research Foundation, Oklahoma City, OK, United States, ${ }^{4}$ Department of Genetics, Fullerton Genetics Center, Asheville, NC, United States, ${ }^{5}$ Department of Pediatrics, Section of Clinical Genetics and Metabolism, University of Colorado School of Medicine, Aurora, CO, United States, ${ }^{6}$ Clinic of Medical Genetics, IRCCS Instituto Auxologico Italiano, Milan, Italy, ${ }^{7}$ Department of Pediatrics, Clinical Genetics, Levine Children's Hospital at Carolina Healthcare System, Charlotte, NC, United States, ${ }^{8}$ GeneDx, Gaithersburg, $M D$, United States, ${ }^{9}$ Institute of Human Genetics, Galilee Medical Center, Nahariya, Israel, ${ }^{10}$ The Azrieli School of Medicine, Bar-Ilan University, Safed, Israel, ${ }^{11}$ Department of Pediatrics, UT Health Medical School, Houston, TX, United States, ${ }^{12}$ Institute of Human Genetics, University of Lübeck, Lübeck, Germany, ${ }^{13}$ Laboratorio di Genetica Medica, ASST Papa Giovanni XXIII, Bergamo, Italy, ${ }^{14}$ Mendelics Genomic Analysis, Sao Paulo, Brazil, ${ }^{15}$ Department of Neurology, Kennedy Krieger Institute, The Johns Hopkins University School of Medicine, Baltimore, MD, United States, ${ }^{16}$ Department of Pediatrics, University Hospital Münster, Münster, Germany, ${ }^{17}$ Department of Pediatrics, Zuyderland Medical Center, Heerlen and Sittard, Netherlands, ${ }^{18} \mathrm{CeGaT} \mathrm{GmbH}$ and Praxis für Humangenetik Tübingen, Tübingen, Germany, ${ }^{19}$ Division of Medical Genetics, Department of Pediatrics, Saint Louis University School of Medicine, Saint Louis, MO, United States, ${ }^{20}$ Division of Clinical Genomics, Ambry Genetics, Aliso Viejo, CA, United States, ${ }^{21}$ Department of Clinical Genetics and School for Oncology and Developmental Biology, Maastricht University Medical Center, Maastricht, Netherlands, ${ }^{22}$ Institute for Clinical Genetics, Carl Gustav Carus Faculty of Medicine, TU Dresden, Dresden, Germany

MAPK8IP3 has been shown to be part of the axonal transport machinery which is essential for the function and maintenance of neurons. Therefore, MAPK8IP3 was an appealing candidate gene for further investigation after the initial identification of a de novo missense variant in an individual with a neurodevelopmental disorder. Through international collaboration and exome sequencing in a cohort that sums up to 27232 affected individuals, we identified heterozygous de novo variants in MAPK8IP3 in thirteen unrelated individuals with an overlapping phenotype of mild to severe intellectual disability and variable brain anomalies such as perisylvian polymicrogyria, cerebral or cerebellar atrophy and hypoplasia of the corpus callosum. De novo variants comprise six missense variants, three of which are recurrent, and three truncating variants. Brain anomalies were consistent among individuals harboring recurrent de novo missense variants, indicating possible variant specific phenotypic effects. We provide several lines of evidence for causation through (1) the significant enrichment of de novo variants in MAPK8IP3 within our cohort, (2) structural modelling of variants that lie in known (template) structures and (3) through using the CRISPRCas9 system to target six conserved amino acid positions in Caenorhabditis elegans to demonstrate increased axonal lysosomal density for two and an adverse locomotion phenotype for five of the six investigated human variants in this model. Taken together, we firmly establish heterozygous de novo variants in MAPK8IP3 as a novel cause of a neurodevelopmental disorder with intellectual disability and variable brain anomalies.

K. Platzer: None. H. Sticht: None. S.L. Edwards: None. W. Allen: None. K.M. Angione: None. M.T. Bonati: None. C. Brasington: None. M.T. Cho: None. L. A. Demmer: None. T. Falik-Zaccai: None. C.N. Gamble: None. Y. Hellenbroich: None. M. Iascone: None. F. Kok: None. S. Mahida: None. H. Mandel: None. T. Marquardt: None. K. McWalter: None. B. Panis: None. A. Pepler: None. H. Pinz: None. L. Ramos: None. D.N. Shinde: None. C. Smith-Hicks: None. A.P.A. Stegmann: None. P. Stöbe: None. C.T.R.M. Stumpel: None. C. Wilson: None. J.R. Lemke: None. N. Di Donato: None. K.G. Miller: None. R. Abou Jamra: None.

\section{C12.5}

Defective DNA polymerase a-primase leads to $X$-linked intellectual disability associated with severe growth retardation, microcephaly and hypogonadism

\section{H. Van Esch ${ }^{1}$, R. Colnaghi ${ }^{2}$, K. Freson ${ }^{3}$, P. Starokadomskyy ${ }^{4}, A . Z^{2} a n k l^{5}, L . B a c k x^{1}$, I. Abramowicz ${ }^{2}$, E. Outwin ${ }^{2}$, L. Rohena ${ }^{6}$, C. Faulkner ${ }^{7}$, G. Leong $^{8}$, R. Newbury-Ecob ${ }^{9}$, R. Challis $^{10}$, K. Ounap ${ }^{11}$, P. Witters ${ }^{12}$, E. Seuntjens ${ }^{13}$, K. Devriendt ${ }^{1}$, E. Burstein ${ }^{4}$, K. Low', M. O'Driscoll ${ }^{2}$}

${ }^{1}$ Center for Human Genetics, LEUVEN, Belgium, ${ }^{2}$ Genome Damage \& Stability Centre, University of Sussex, Brighton, United Kingdom, ${ }^{3}$ Center for Molecular and Vascular Biology, LEUVEN, Belgium, ${ }^{4}$ University of Texas Southwestern Medical Center, Dallas, TX, United States, ${ }^{5}$ The Children's Hospital at Westmead, Westmead, Australia, ${ }^{6}$ Department of Pediatrics, San Antonio Military Medical 
Center, San Antonio, TX, United States, ${ }^{7}$ Bristol Genetics Laboratory, Southmead Hospital, Bristol, United Kingdom, ${ }^{8}$ Department of Paediatrics, Nepean Hospital, Kingswood, Australia, ${ }^{9}$ Clinical Genetics, St Michaels Hospital, Bristol, United Kingdom, ${ }^{10}$ Institute of Genetics and Molecular Medicine, University of Edinburgh, Edinburgh, United Kingdom, ${ }^{11}$ Tartu University Hospital and Institute of Clinical Medicine, Tartu, Estonia, ${ }^{12}$ Center for Metabolic Diseases, LEUVEN, Belgium, ${ }^{13}$ Developmental Neurobiology, KU Leuven, LEUVEN, Belgium

Replicating the human genome efficiently and accurately is a daunting challenge, involving the duplication of upwards of three billion base pairs. At the core of the complex machinery that achieves this task are three members of the B family of DNA polymerases; DNA polymerase $\alpha, \delta$ and $\varepsilon$. Collectively these multimeric polymerases ensure DNA replication proceeds at optimal rates approaching $2 \times 10^{3}$ nucleotides/min, and with an error rate of less than one per million nucleotides polymerised. The majority of DNA replication of undamaged DNA is conducted by DNA polymerases $\delta$ and $\varepsilon$. DNA polymerase $\alpha$-primase complex performs limited synthesis to initiate the replication process, along with Okazaki fragment synthesis on the discontinuous lagging strand. An increasing number of human disorders caused by defects in different components of the DNA replication apparatus have been described to date. These are clinically diverse, involving a wide range of features including variable combinations of growth delay, immunodeficiency, endocrine insufficiencies, lipodystrophy and cancer predisposition. Here, using various complementary approaches including classical linkage analysis, targeted next generating sequencing and whole exome sequencing, we describe distinct missense and splice-impacting mutations in POLAl in five unrelated families presenting with an $\mathrm{X}$-linked syndrome involving intellectual disability, proportionate short stature, microcephaly and hypogonadism. POLAl encodes the p180 catalytic subunit of DNA polymerase $\alpha$-primase. A range of replicative impairments could be demonstrated in patient-derived lymphoblastoid cell lines. Our findings describe the presentation of pathogenic mutations in a catalytic component of a $B$ family DNA polymerase member; DNA polymerase $\alpha$.

H. Van Esch: None. R. Colnaghi: None. K. Freson: None. P. Starokadomskyy: None. A. Zankl: None. L. Backx: None. I. Abramowicz: None. E. Outwin: None. L. Rohena: None. C. Faulkner: None. G. Leong: None. R. Newbury-Ecob: None. R. Challis: None. K. Ounap: None. P. Witters: None. E. Seuntjens: None. K. Devriendt: None. E. Burstein: None. K. Low: None. M. O'Driscoll: None.

\section{C12.6}

Non-penetrance of a frameshifting SHANK3 deletion is associated with compensatory mechanisms in both alleles

\section{B. Haukanes, T. Nordtveit, G. Houge}

Department of Medical Genetics, Bergen, Norway

In three brothers with severe learning problems, mild ID, and variable degrees of autism, we found an early frameshifting and maternally derived SHANK3 deletion, (NM_033517.1):c.1030+2929_1688+71delinsG p. (Val344AlafsTer71), removing $10-13$ of the 22 exons. The non-mosaic SHANK3 deletion carrier mother is in regular work without learning difficulties or other problems.

To explore how the mother can be non-penetrant, we collected blood and fibroblast cells from all family members for epigenetic-, RNA- and protein studies. In fibroblasts, deep sequencing-based mRNA quantification indicated that the mother had a 3.5 fold higher SHANK3 mRNA expression from the normal allele than her sons, possibly related to an enhancer triplication $17.5 \mathrm{~kb}$ upstream only detected in the mother. This enhancer triplication has a population prevalence of $20 \%$ (561/2771). A more pronounced difference was found for the terminal SHANK3 exons 17-22 also included in two terminal isoforms (isoE/Promoter5 and isoF/Promoter6) located downstream of the deletion. To investigate the epigenetic status of the $\mathrm{CpG}$ islands related to the SHANK3 promoters P1-P6, we performed methylation-sensitive amplicon based deep sequencing of DNA purified from blood and fibroblasts after bisulphite treatment. The putative isoF promoter (core Promoter6 w/ nine $\mathrm{CpGs})$ was hypomethylated $(\bar{x}=1.5 \pm 0.94 \%)$ in fibroblast-DNA from all deletion carriers compared to control subjects $(\bar{x}=15.5 \pm 0.74 \%)$.

In conclusion, non-penetrance of an early SHANK3 deletion in a carrier mother is associated with $i$ ) higher SHANK3 expression from her normal allele, possibly related to a common enhancer triplication only in the mother, and ii) increased expression of C-terminal SHANK3 isoforms from both alleles.

B. Haukanes: None. T. Nordtveit: None. G. Houge: None.

\section{C13 Pharmacogenomics}

\section{C13.1}

Metabolomic consequences of PCSK9 inhibition compared with statin therapy

\section{P. Würtz, S. Ruosaari}


Nightingale Health Ltd., Helsinki, Finland

Introduction: Both statins and PCSK9 inhibitors lower blood low-density lipoprotein cholesterol (LDL-C) levels to reduce risk of cardiovascular events. We performed detailed metabolic profiling of a large randomised statin trial and compared the results with the effects of genetic inhibition of PCSK9, acting as a naturally occurring trial.

Materials and Methods: Altogether 228 circulating metabolic measures were quantified by nuclear magnetic resonance spectroscopy, including lipoprotein subclass concentrations and their lipid composition, fatty acids, and amino acids, for 5,359 individuals in the PROSPER trial. The corresponding metabolic measures were analysed in eight cohorts $(\mathrm{N}=72,185)$ using PCSK9 rs11591147 to mimic the therapeutic effects of PCSK9 inhibitors.

Results: Scaled to an equivalent lowering of LDL-C, the effects of genetic inhibition of PCSK9 on 228 metabolic markers were generally consistent with those of statin therapy $\left(R^{2}=0.88\right)$. However, discrepancies were observed for very-low-density lipoprotein (VLDL) lipid measures. For instance, genetic inhibition of PCSK9 had weaker effects on lowering of VLDL-cholesterol compared with statin therapy (54 vs. $77 \%$ reduction, relative to the lowering effect on LDL-C). Genetic inhibition of PCSK9 showed no significant effects on amino acids, ketones, or a marker of inflammation, whereas statin treatment weakly lowered GlycA levels.

Conclusion: Genetic inhibition of PCSK9 had similar metabolic effects as statin therapy on detailed lipid and metabolite profiles. However, PCSK9 inhibitors are predicted to have weaker effects on VLDL lipids compared with statins for an equivalent lowering of LDL-C, which potentially translate into smaller reductions in cardiovascular disease risk.

P. Würtz: A. Employment (full or part-time); Significant; Nightingale Health Ltd. S. Ruosaari: A. Employment (full or part-time); Significant; Nightingale Health Ltd.

\section{C13.2}

Longitudinal analysis of the gut microbiome reveals dynamic changes in relation to medications \& phenotypes

\section{Chen ${ }^{1,2}$, S. Garmaeva ${ }^{1}$, A. Kurilshikov ${ }^{1}$, R. Gacesa ${ }^{3}$,} A. Vich Vila ${ }^{3}, R$. Weersma ${ }^{3}, C$. Wijmenga ${ }^{1,4}$, A. Zhernakova ${ }^{1}, J . F^{1,2}$

${ }^{1}$ Department of Genetics, University Medical Center Groningen, Groningen, Netherlands, ${ }^{2}$ Department of Pediatrics, University Medical Center Groningen, Groningen, Netherlands, ${ }^{3}$ Department of Gastroenterology and Hepatology, University Medical Center Groningen,
Groningen, Netherlands, ${ }^{4}$ Jebsen Coeliac Disease Research Centre, University of Oslo, Oslo, Norway

Interaction between the human genome and gut microbiome is vital to human health. While the human genome is set at birth, the gut microbiome can undergo dynamic changes over the course of an individual's life. However, we still know little about temporal shifts in the human gut microbiome, nor about the causes and consequences of temporal shifts. We performed a longitudinal analysis on the gut microbiome of 341 participants in the Dutch population-based cohort Lifelines-DEEP, each individual having metagenomics and deep phenotypic data at two time points ( $\sim 5$ years apart). Significant temporal changes in the gut microbiome were detected at the levels of microbial composition, function and antibiotic resistance. Abundance difference was observed for $40 \%$ of taxonomies, $60 \%$ of functional pathways and $40 \%$ of antibiotic resistance genes. Furthermore, genetic stability analysis at strain level has revealed several species are under high evolution rate, including gastrointestinal disease associated Ruminococcus torques. Notable, temporal changes were associated to individual phenotypic variation and lifestyle factors. For instance, the genetic variation in Coprococcus sp ART55/ 1 strains was higher in individuals with larger changes in BMI, while Streptococcus thermophilus and Bifidobacterium longum showed higher mutation rates in proton pump inhibitor and non-steroidal anti-inflammatory users, as compared to non-users. Our data show that the gut microbiome sees dynamic changes not only in microbial composition and functional profiles but also in microbial genetic variation and antibiotic resistance. Our findings yield novel insights into the gut microbiome's impact on the development of complex diseases and traits over time.

L. Chen: None. S. Garmaeva: None. A. Kurilshikov: None. R. Gacesa: None. A. Vich Vila: None. R. Weersma: None. C. Wijmenga: None. A. Zhernakova: None. J. Fu: None.

C13.3

Lifelong genetically lowered sclerostin and risk of cardiovascular disease

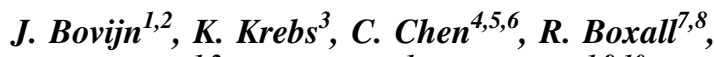

J. C. Censin ${ }^{1,2}$, T. Ferreira ${ }^{1}$, S. L. Pulit ${ }^{1,9,10}$,

C. A. Glastonbury', S. Laber ${ }^{1,2}$, I. Y. Millwood ${ }^{7,8}$, K. Lin ${ }^{7}$, L. $\mathrm{Li}^{11}$, Z. Chen ${ }^{7}$, L. Milani ${ }^{3}$, R. G. Walters ${ }^{7,8}$, R. Mägi ${ }^{3}$, B. M. Neale ${ }^{4,6}$, C. M. Lindgren ${ }^{1,2,9,12}$, M. V. Holmes ${ }^{1,7,8,12}$

${ }^{1}$ Big Data Institute, University of Oxford, Oxford, United Kingdom, ${ }^{2}$ Wellcome Centre for Human Genetics, Nuffield Department of Medicine, University of Oxford, Oxford, United Kingdom, ${ }^{3}$ Estonian Genome Center, Institute of 
Genomics, University of Tartu, Tartu, Estonia, ${ }^{4}$ Analytic and Translational Genetics Unit, Massachusetts General Hospital, Boston, MA, United States, ${ }^{5}$ Psychiatric and Neurodevelopmental Genetics Unit, Boston, MA, United States, ${ }^{6}$ Stanley Center for Psychiatric Research, Broad Institute of MIT and Harvard, Cambridge, MA, United States, ${ }^{7}$ Clinical Trial Service Unit and Epidemiological Studies Unit (CTSU), Nuffield Department of Population Health, University of Oxford, Oxford, United Kingdom, ${ }^{8}$ Medical Research Council Population Health Research Unit (MRC PHRU), Nuffield Department of Population Health, University of Oxford, Oxford, United Kingdom, ${ }^{9}$ Program in Medical and Population Genetics, Broad Institute, Cambridge, MA, United States, ${ }^{10}$ Department of Genetics, University Medical Center Utrecht, Utrecht, Netherlands, ${ }^{11}$ Department of Epidemiology \& Biostatistics, Peking University Health Science Centre, Peking University, Bejing, China, ${ }^{12}$ National Institute for Health Research Oxford Biomedical Research Centre, Oxford University Hospitals NHS Foundation Trust, John Radcliffe Hospital, Oxford, United Kingdom

Introduction: Inhibition of sclerostin is a novel therapeutic approach to lowering fracture risk. However, phase III randomised controlled trials (RCTs) of romosozumab, a sclerostin inhibitor, suggest an imbalance of serious cardiovascular events.

Methods: We used two independent variants in SOST (encoding sclerostin) associated with bone mineral density (BMD) as proxies for therapeutic inhibition of sclerostin. We estimated the effects on risk of osteoporosis, fracture, coronary heart disease (CHD) and a further 22 cardiometabolic traits, by combining data from 478,967 participants from three cohorts and up to $1,030,836$ participants from nine GWAS consortia. In addition, we performed metaanalysis of cardiovascular outcomes from RCTs of romosozumab.

Results: RCT meta-analysis identified a higher risk of cardiac ischaemic events in patients randomised to romosozumab (25 events among 4,298 individuals; odds ratio [OR] 2.98; 95\% confidence interval [CI], 1.18-7.55; $\mathrm{P}=$ 0.017). Scaled to the equivalent dose of romosozumab $\left(210 \mathrm{mg} / \mathrm{month} ; 0.09 \mathrm{~g} / \mathrm{cm}^{2}\right.$ higher BMD), the $S O S T$ variants associated with lower risk of fracture (OR, 0.59; $95 \%$ $\mathrm{CI}, 0.54-0.66 ; \mathrm{P}=1.4 \times 10^{-24}$ ), and osteoporosis (OR, $\left.0.43 ; 95 \% \mathrm{CI}, 0.36-0.52 ; \mathrm{P}=2.4 \times 10^{-18}\right)$. The SOST variants associated with higher risk of myocardial infarction and/or coronary revascularisation $(69,649$ cases; OR, 1.18 ; 95\% CI, 1.06-1.32; $\mathrm{P}=0.003)$ and type 2 diabetes (OR 1.15 ; 95\% CI, 1.05-1.27; $\mathrm{P}=0.003)$, higher systolic blood pressure $\left(1.3 \mathrm{mmHg} ; 95 \% \mathrm{CI} 0.8-1.9 ; \mathrm{P}=5.9 \times 10^{-6}\right)$ and waist-to-hip-ratio adjusted for BMI (0.05 SDs; $95 \% \mathrm{CI}$, $0.02-0.08 ; \mathrm{P}=8.5 \times 10^{-4}$ ).
Conclusion: Genetically and therapeutically lowered sclerostin leads to higher risk of cardiovascular events. Rigorous evaluation of the cardiovascular safety of romosozumab and other sclerostin inhibitors is warranted.

J. Bovijn: None. K. Krebs: None. C. Chen: None. R. Boxall: None. J.C. Censin: None. T. Ferreira: None. S.L. Pulit: None. C.A. Glastonbury: None. S. Laber: None. I. Y. Millwood: None. K. Lin: None. L. Li: None. Z. Chen: None. L. Milani: None. R.G. Walters: None. R. Mägi: None. B.M. Neale: None. C.M. Lindgren: None. M.V. Holmes: None.

\section{C13.4}

Advanced renal cancer patients with tumor KDM5C mutations show improved response to anti-angiogenic therapy

M. Santos ${ }^{1}$, J. Roldan-Romero ${ }^{1}$, J. Lanillos ${ }^{I}$, F. García ${ }^{1}$, B. Calsina ${ }^{1}$, M. Pulgarín ${ }^{1}$, Á. Martínez ${ }^{1}$, R. Letón ${ }^{1}$, C. Montero-Conde ${ }^{I}$ A. Cascón ${ }^{1,2}$, M. Robledo ${ }^{1,2}$, B. Beuselinck ${ }^{3}$, J. García-Donas $^{4}$, C. Rodríguez-Antona ${ }^{1,2}$

${ }^{1}$ Spanish National Cancer Research Centre, Madrid, Spain, ${ }^{2}$ Centro de Investigación Biomédica en Red de Enfermedades Raras, Madrid, Spain, ${ }^{3}$ University of Leuven, Leuven, Belgium, ${ }^{4}$ Clara Campal Comprehensive Cancer Center, Madrid, Spain

Introduction: Survival of metastatic renal cell carcinoma (mRCC) has doubled in the last decades with the approval of targeted drugs and recently immunotherapies. However, responses are very heterogeneous and we lack predictive biomarkers. Anti-angiogenic therapies are key first-line drugs in mRCC. Our objective was to identify mutations associated with angiogenesis inhibitors response that could help personalizing mRCC treatment.

Material and Methods: Samples were collected through an observational study from the Spanish Oncology Genitourinary Group and Leuven Hospital. Whole exome sequencing (WES) was performed on tumor and blood from 28 mRCC patients with extreme response to first-line sunitinib. 45 genes were sequenced on 156 additional tumor cases. Patients were classified as anti-angiogenic drug responders (PFS $>18$ months), non-responders $(\mathrm{PFS}<$ 6 months) or intermediate-responders (remaining patients). A KDM5C knockout (KO) model in 786-O cells was generated using CRISPR/Cas9 and characterized by proteomics (iTRAQ technology).

Results: WES revealed that mutations in KDM5C, encoding a chromatin remodeling protein, were overrepresented among responder patients, and full series analysis confirmed the association $(\mathrm{OR}=0.23 ; 95 \% \mathrm{CI}=$ $0.07-0.77 ; \mathrm{P}=0.017$ ). Proteomic analysis in the KDM5C 
cellular model revealed 414 proteins differentially expressed $(\mathrm{FC}=0.3 / 3.3 ; \mathrm{P}=0.01)$. The proangiogenic proteins SPHK2, SRC and RHOC were overexpressed $(\mathrm{P}<0.01)$ in the $\mathrm{KO}$ cells suggesting that mutated tumors are more dependent on angiogenesis.

Conclusion: Mutations in $K D M 5 C$ are associated with improved response to anti-angiogenic treatment in $\mathrm{mRCC}$, potentially due to an overexpression of proangiogenic proteins. Supported by: Spanish Ministry of Economy and Competiveness, an unrestricted educational grant from Pfizer and the Spanish Ministry of Education, Culture and Sport (FPU16/05527).

M. Santos: None. J. Roldan-Romero: None. J. Lanillos: None. F. García: None. B. Calsina: None. M. Pulgarín: None. Á. Martínez: None. R. Letón: None. C. Montero-Conde: None. A. Cascón: None. M. Robledo: None. B. Beuselinck: None. J. García-Donas: None. C. Rodríguez-Antona: None.

\section{C13.5}

Predicting Functional Effects of Missense Variants in Voltage-Gated Sodium and Calcium Channels

H. O. Heyne ${ }^{1}$, S. Iqbal ${ }^{1}$, D. Palmer ${ }^{1}$, K. Johannesen ${ }^{2}$,

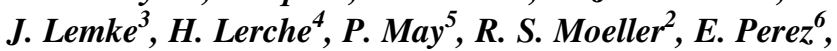
U. Scholl, S. Syrbe ${ }^{8}$, A. J. Campbell, D. Lal', H. Wang ${ }^{1}$, M. J. Daly ${ }^{10}$

${ }^{1}$ Broad Institute of Harvard and MIT, Cambridge, MA, United States, ${ }^{2}$ The Danish Epilepsy Center, Dianalund, Denmark, ${ }^{3}$ University of Leipzig, Leipzig, Germany, ${ }^{4}$ University of Tübingen, Tübingen, Germany, ${ }^{5}$ University of Luxemburg, Luxemburg, Luxembourg, ${ }^{6}$ University of Cologne, Cologne, Germany, ${ }^{7}$ Berlin Institute of Health, Charite Universitaetsmedizin Berlin, Berlin, Germany, ${ }^{8}$ University of Heidelberg, Heidelberg, Germany, ${ }^{9}$ Cleveland Clinic, Cleveland, $\mathrm{OH}$, United States, ${ }^{10}$ Institute for Molecular Medicine Finland (FIMM), Helsinki, Finland

Malfunctions of multiple voltage-gated sodium $\left(\mathrm{Na}_{\mathrm{v}} \mathrm{s}\right.$ encoded by $S C N x A$ genes) and calcium channels $\left(\mathrm{Ca}_{\mathrm{v}} \mathrm{s}\right.$ encoded by CACNA1X genes) have been associated with severe neurological, psychiatric, cardiac and other diseases. In $\mathrm{Na}_{\mathrm{v}} \mathrm{s}$ and $\mathrm{Ca}_{\mathrm{v}} \mathrm{s}$, altered channel activity is frequently grouped into gain or loss of ion channel function (GOF or LOF, respectively). This can lead to specific diseases, e.g. SCN5A LOF variants to Brugada syndrome and SCN5A GOF variants to Long QT syndrome. Based on 19 known gene-disease-mechanisms, we infer LOF (521 variants) and GOF (304 variants) of likely pathogenic variants from disease phenotypes of variant carriers.

We show regional clustering of inferred GOF and LOF variants, respectively, across the alignment of the entire gene family, suggesting shared pathomechanisms in SCN $x A / C A C N A 1 x$ genes. We then use sequence- and structure-based features (not including gene names or positions) to develop a machine learning method that can predict whether a missense variant results in a LOF or GOF associated disease phenotype $(\mathrm{ROC}=0.85)$. We validate the GOF and LOF effect prediction on functionally tested variants in SCN1A, SCN2A and SCN8A $(\mathrm{ROC}=0.83)$ and in large exome-wide datasets of individuals with diseases like autism and epilepsy. Our work also has implications for disease modeling and mechanisms of ion channels. Ultimately, functional prediction of missense variants in these clinically relevant genes may enhance precision medicine in clinical practice.

This work was supported by German Research Foundation stipends HE7987/1-1 and HE7987/1-2 awarded to $\mathrm{HOH}$.

H.O. Heyne: None. S. Iqbal: None. D. Palmer: None. K. Johannesen: None. J. Lemke: None. H. Lerche: None. P. May: None. R.S. Moeller: None. E. Perez: None. U. Scholl: None. S. Syrbe: None. A.J. Campbell: None. D. Lal: None. H. Wang: None. M.J. Daly: None.

C13.6

Taurine supplementation as a potential therapy for progressive retinal degeneration due to biallelic pathogenic variants in the Taurine transporter SLC6A6

E. Ranza ${ }^{1,2,3}$, M. Ansar ${ }^{1}$, M. Shetty ${ }^{4}$ S. A. Paracha ${ }^{5}$,

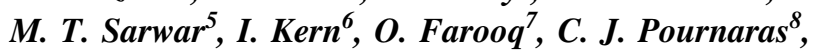
A. Malcles $^{9}$, F. A. Santoni ${ }^{1,10}$, P. Makrythanasis ${ }^{1,11}$, J. Ahmed ${ }^{5}$, K. Henry ${ }^{4}$, S. E. Antonarakis ${ }^{1,2,12}$

${ }^{1}$ Department of Genetic Medicine and Development, University of Geneva, Geneva, Switzerland, ${ }^{2}$ Service of Genetic Medicine, University Hospitals of Geneva, Geneva, Switzerland, ${ }^{3}$ current address, Medigenome, Swiss Institute of Genomic Medicine, Geneva, Switzerland, ${ }^{4}$ Dept. of Biomedical Sciences, School of Medicine and Health Sciences, University of North Dakota, Grand Forks, ND, United States, ${ }^{5}$ Institute of Basic Medical Sciences, Khyber Medical University, Peshawar, Pakistan, ${ }^{6}$ Pediatric Nephrology and Metabolism Unit, Pediatric Subspecialties Service, Children's Hospital, Geneva University Hospitals, Geneva, Switzerland, ${ }^{7}$ Bahria University Medical and Dental College, Karachi, Pakistan, ${ }^{8}$ Hirslanden Clinique La Colline, Geneva, Switzerland, ${ }^{9}$ Department of Ophthalmology, University Hospitals of Geneva, Geneva, Switzerland, ${ }^{10}$ Department of Endocrinology Diabetes and Metabolism, University hospital of Lausanne, Lausanne, Switzerland, ${ }^{11}$ Biomedical Research Foundation of the Academy of Athens, Athens, Greece, ${ }^{12} i G E 3$ Institute of Genetics and Genomics of Geneva, Geneva, Switzerland 
We have studied a Pakistani consanguineous family with two children suffering from progressive visual impairment and identified a pathogenic homozygous missense variant Gly399Val in the $8^{\text {th }}$ transmembrane domain of SLC6A6. 3D modeling of this variant has indicated that it likely causes displacement of the Tyr138 (TM3) side chain, important for transport of taurine. The two affected children exhibited very low blood taurine levels ( 6 and $7 \mu \mathrm{mol} / \mathrm{l})$, heterozygous parents had intermediate levels (24 and $34 \mu \mathrm{mol} / \mathrm{l})$, and an unaffected non-carrier sibling had normal levels $(71 \mu \mathrm{mol} / \mathrm{l})$ (normal values:37-127 $\mu \mathrm{mol} / \mathrm{l})$. In-vitro experiments showed a reduced $\mathrm{K}_{\mathrm{M}}$ and $\mathrm{V}_{\mathrm{MAX}}$ for taurine uptake by HEK-293 cells expressing the mutant (Gly399Val) SLC6A6 providing only $11-18 \%$ of transport compared to wild type SLC6A6. Similar results were obtained with fibroblasts derived from affected individuals. Clinical evaluation showed that the affected boy has complete visual loss, while the affected girl retained some visual acuity. In addition, both affected siblings have mild hypokinetic cardiomyopathy with systolic dysfunction. Slc6a6 knockout in a previously reported mouse model causes progressive retinal degeneration, cardiomyopathy and very low taurine levels in blood and other tissues. Following ethics approval from the Swiss authorities we have initiated a taurine supplementation to both affected individuals. Clinical examinations performed after one year of taurine supplementation $(100 \mathrm{mg} / \mathrm{kg} / \mathrm{day}$, in 3 doses $)$ showed normal taurine levels in blood and no progression of retinal degeneration in the affected girl.

We conclude that the supplementation of taurine could stop the progressive visual loss caused by hypomorphic functional defect in the SLC6A6 transporter in humans.

E. Ranza: None. M. Ansar: None. M. Shetty: None. S. A. Paracha: None. M.T. Sarwar: None. I. Kern: None. O. Farooq: None. C.J. Pournaras: None. A. Malcles: None. F.A. Santoni: None. P. Makrythanasis: None. J. Ahmed: None. K. Henry: None. S.E. Antonarakis: None.

\section{C14 Genetic counselling developments}

\section{C14.1}

Effect of genetic counseling on adherence to psychotropic medication in people with serious mental illness

\author{
J. Austin ${ }^{1}$, E. Morris ${ }^{1}$, R. Batallones ${ }^{1}$, P. Carrion ${ }^{1}$, \\ C. Slomp ${ }^{1}$, J. Ryan', A. Albert ${ }^{2}$ \\ ${ }^{1}$ University of British Columbia, Vancouver, BC, Canada, \\ ${ }^{2}$ Women's Health Research Institute, Vancouver, BC, \\ Canada
}

Introduction: In the context of serious mental illness (SMIs, i.e. schizophrenia, bipolar disorder, and schizoaffective disorder) medication non-adherence is an important and complex problem, for which no effective gold standard solution exists. Beliefs about illness etiology influence behaviours like medication adherence, thus genetic counseling (GC), which helps people understand illness etiology and how it relates to recommended treatments could plausibly influence medication adherence.

Materials and Methods: We recruited 110 people with SMI to a study designed to test the hypothesis that GC would increase adherence to psychotropic medications. Participants completed the Brief Adherence Rating Scale (BARS) twice (1month apart) before receiving GC (T1: baseline and T2: immediately pre-GC) and twice after GC (T3 and T4, 1 and 2 months post-GC respectively). We used mixed effects logistic regression and validated criteria to model BARS scores as a binary variable (adherent $=\mathrm{Y} / \mathrm{N})$ over the 4 time-points, and accounted for repeated measures.

Results: Using 392 observations from 108 participants (schizophrenia $\mathrm{n}=23$, schizoaffective $\mathrm{n}=14$, bipolar $\mathrm{n}=$ 71), there was a significant relationship between the probability of adherence and timepoint $(\mathrm{p}=0.031)$. Post-hoc pairwise comparisons with holm-corrected p-values indicated the probability of adherence was higher at $\mathrm{T} 4(86 \%)$ compared to $\mathrm{T} 2(78 \%)(\mathrm{p}=0.04, \mathrm{OR}=7.08(95 \%$ CI 1.73 to 28.99)).

Conclusion: Our data suggest that GC can improve adherence to psychotropic medications in individuals with SMI and that the effect size is large. Further work on the impact of GC on medication adherence, as well as mental health and economic outcomes is warranted. Supported by Pfizer Canada.

J. Austin: B. Research Grant (principal investigator, collaborator or consultant and pending grants as well as grants already received); Significant; Pfizer Canada. E. Morris: None. R. Batallones: None. P. Carrion: None. C. Slomp: None. J. Ryan: None. A. Albert: None.

\section{C14.2}

Psychiatric Genetic Counselling: Efficacy of training and implications for practice
K. A. McGhee ${ }^{1}$ M. Watson ${ }^{2}$, A. Inglis ${ }^{3}$, E. Morris $^{3}$, R. Moldovan ${ }^{4,5}$, J. C. Austin ${ }^{3}$
${ }^{I}$ Faculty of Science \& Technology, Bournemouth Univer- sity, POOLE, United Kingdom, ${ }^{2}$ Wessex Clinical Genetics Service, University Hospital Southampton NHS Foundation Trust, Southampton, United Kingdom, ${ }^{3}$ University of British Columbia, Departments of Psychiatry \& Medical Genetics, 
Vancouver, BC, Canada, ${ }^{4}$ Babes Bolyai University, ClujNapoca, Romania, ${ }^{5}$ University of Manchester, Manchester, United Kingdom

Introduction: Accompanying our growing understanding of the complex nature of major psychiatric disorders is a parallel need to assist patients and families in understanding the mechanisms and adapting to the implications of psychiatric disorders, including risk factors that might contribute to illness occurrence in families. Psychiatric genetic counselling (PGC) is not routinely offered, with a few exceptions. In order to address patients and professionals interests and needs, we designed and implemented a training course aimed at equipping them with theoretical knowledge and practical skills. The impact of the course was assessed.

Method: Data was collected from five free training courses involving 125 professionals from 18 countries, between 2015 and 2019. Responses were anonymised. Questionnaires assessing participants knowledge and self efficacy were filled out before and after the training.

Results: There was a significant increase in both knowledge $[\mathrm{t}(124)=2.05, \mathrm{p}<.004)]$ and self-efficacy $[\mathrm{t}$ $(124)=27.1, p<001]$ following the course, suggesting participants not only gained knowledge and skills during the course but also felt more empowered to offer PGC after the course.

Conclusion: Adapting existing services or designing new ones will take time, requiring further empirical evidence to show the impact and implications of PGC training. Establishing practice recommendation and standardised training guidelines that include a good understanding of psychiatric disorders and genomics, a proficient mastery of counselling skills and ethical standards is clearly timely and essential in order to ensure quality of future PGC services.

K.A. McGhee: None. M. Watson: None. A. Inglis: None. E. Morris: None. R. Moldovan: None. J.C. Austin: None.

\section{C14.3}

Large scale group genetic counselling: a novel service delivery model

\section{Z. Lohn ${ }^{1,2}$, J. Nuk ${ }^{1,2}$, A. Fok ${ }^{3}$, M. Richardson ${ }^{4}$, S. Mung ${ }^{1}$,} J. Yuson ${ }^{1}$, M. Jevon ${ }^{1}$, K. Schrader ${ }^{1,5}$, S. Sun ${ }^{1,6}$

${ }^{1}$ BC Cancer Hereditary Cancer Program, Vancouver, BC, Canada, ${ }^{2}$ Department of Medical Genetics, University of British Columbia, Vancouver, BC, Canada, ${ }^{3}$ Canada's Michael Smith Genome Sciences Centre, BC Cancer, Vancouver, BC, Canada, ${ }^{4}$ Department of Interdisciplinary Oncology, University of British Columbia, Vancouver, BC, Canada, ${ }^{5}$ Department of Molecular Oncology, University of
British Columbia, Vancouver, BC, Canada, ${ }^{6}$ Division of Medical Oncology, University of British Columbia, Vancouver, BC, Canada

Introduction: Novel streamlined models for genetic counselling are being developed in response to increasing demand for cancer genetic services. To improve access and decrease wait times, we are trialing large scale group genetic counselling in a publicly funded population-based health care setting.

Methods: This initiative compares pre-test group genetic counselling sessions (up to 50 patients per group) to traditional one-on-one genetic counselling. A secondary objective is to trial genetic testing in unaffected individuals. Patients are provided the option of either the large group arm or the traditional arm with the aim to enroll 400 patients in total. All patients receive a post-test one-on-one results session. Eligibility includes unaffected adult individuals with a family history meeting clinical testing criteria. Patients are invited to complete the Genetic Counselling Outcome Scale, a satisfaction survey and the Multidimensional Impact of Cancer Risk Assessment.

Results: To date, 344 patients (176 group; 168 one-onone) have been enrolled in the trial. Of the 174 patients who have completed testing, $15(8.6 \%)$ have had a pathogenic/ likely pathogenic variant identified, most commonly in BRCA1/BRCA2. Both the large group arm and traditional one-on-one arm have shown high patient satisfaction.

Conclusion: Emerging data shows that a large scale group genetic counselling model is feasible, effective and acceptable to patients; this model may be included in a multi-pronged approach to deliver quality hereditary cancer care while balancing the needs of the patient, the provider, and the public medical system.

Z. Lohn: None. J. Nuk: None. A. Fok: None. M. Richardson: None. S. Mung: None. J. Yuson: None. M. Jevon: None. K. Schrader: None. S. Sun: None.

\section{C14.4}

Genetic counselling experience in Iceland of web-based return of $\mathrm{BRCA2}$ research results

V. Stefansdottir, E. T. Thorolfsdottir, B. B. Gunnarsdottir, A. Ulfarsdottir, T. Jonsdottir, H. B. Hognason, J. J. Jonsson

Landspitali National University Hospital, Reykjavik, Iceland

Increasingly, genetic results from research are being returned to study participants, usually with their prior knowledge and consent. deCODE Genetics, a genetic research company has genotypic and medical data from 
more than 160.000 Icelanders. The company opened the website arfgerd.is, on May 15th 2018 where research participants can query their genotype regarding the Icelandic pathogenic founder variant (PV) BRCA2: c.771_775del5. Positive individuals are advised to contact the genetic counselling unit (GC) at Landspitali-National University Hospital for confirmatory testing and further advise.

We report the experience of the GC up to the end of 2018. At the time, 44.300 individuals had signed onto the website. A total of 340 (average age 44 years), had received positive results. We estimate that at least 40 of the 340 had received their results and genetic counselling earlier. A total of 175 of the 300 remaining had contacted the GC unit by 31.12.2018. The remaining $125(40 \%)$ had not but the reason is not clear. Additionally, 118 relatives contacted the GC unit. Of the BRCA2 positive $(\mathrm{n}=175)$, (66) $37 \%$ knew about the PV in the family while 95 (54\%) did not. Most were grateful for the knowledge. Surprised about the results were 46 (24\%), and not 61 (29\%). Worried, scared, upset or had difficulties coping with the positive results were 49 (29\%), and $13 \%$ not. The increased need for counselling and surveillance was significant, especially given the small size of the units involved. This emphasizes the importance of collaboration and consultation when such projects are launched.

V. Stefansdottir: None. E.T. Thorolfsdottir: None. B. B. Gunnarsdottir: None. A. Ulfarsdottir: None. T. Jonsdottir: None. H.B. Hognason: None. J.J. Jonsson: None.

\section{C14.5}

The making of the BRCA-chatbot - A patient centered digital counselling tool to support individuals undergoing genetic testing for hereditary breast and ovarian cancer

\section{E. Siglen ${ }^{1,2}$, H. Høberg-Vetti, ${ }^{2,1}$, M. Tveit Haavind ${ }^{2}$, V. Steen ${ }^{3,1}$, A. Hamang 4 , S. Tronsli Nergård ${ }^{5}$, N. Strømsvik , T. Akselberg Hatlebrekke $^{7}$, H. Skarbø ${ }^{8}$, C. Bjorvatn ${ }^{1,2,3}$}

${ }^{I}$ Department of Medical Genetics, Haukeland University Hospital, Bergen, Norway, ${ }^{2}$ Western Norway Familial Cancer Center, Bergen, Norway, ${ }^{3}$ Department of Clinical Science, University of Bergen, Bergen, Norway, ${ }^{4}$ Department of Medical Genetics, St. Olavs Hospital, Trondheim University Hospital, Trondheim, Norway, ${ }^{5}$ Department of Medical Genetics, Oslo University Hospital, Oslo, Norway, ${ }^{6}$ Familial Cancer Center, Department of Medical Genetics, University Hospital of North Norway, Troms $\phi$, Norway, ${ }^{7}$ Helse Vest IKT, Department for business development,
Haukeland University Hospital, Bergen, Norway, ${ }^{8}$ Digital Culture, University of Bergen, Bergen, Norway

Introduction: Nearly $50 \%$ of patients with breast cancer meet the current Norwegian BRCA1/2-testing criteria, requiring the health care system to develop sustainable solutions for genetic testing and counselling. As the capacity to meet the increased demand for personalized counselling is limited, we need new information and communication tools for this purpose.

Aim: Design and implement chatbot technology as an easy, reliable and safe communication tool to support individuals undergoing genetic testing for hereditary breast cancer.

Materials and methods: We are developing and implementing a new app that can perform chat conversations about genetic testing with patients, using so-called Chatbot technology, based on artificial intelligence. User experiences related to trust and usefulness of the Chatbot is central to the study, and will be evaluated through usability testing and focus group interviews. Results from the first pilot-test will be presented.

Discussion: The use of Chatbots in health care is emerging, thus the project is expected to be timely. We expect that this BRCA-Chatbot will serve as an important proof-ofprinciple concept and public innovation process, to supplement and improve traditional genetic counselling. The BRCA-Chatbot may personalize counselling and put the patients in a proactive role in their search for information. We anticipate that the patients using the BRCA-Chatbot will be better prepared for face-to-face consultations in the hospital, and that the tool will be a useful and trusted aid in mastering their lives after these consultations. The study is funded by the Norwegian Cancer Society and the Norwegian Breast Cancer Society.

E. Siglen: B. Research Grant (principal investigator, collaborator or consultant and pending grants as well as grants already received); Significant; The Norwegian Breast Cancer Society. H. Høberg-Vetti: None. M. Tveit Haavind: None. V. Steen: None. A. Hamang: None. S. Tronsli Nergård: None. N. Strømsvik: None. T. Akselberg Hatlebrekke: None. H. Skarbø: None. C. Bjorvatn: B. Research Grant (principal investigator, collaborator or consultant and pending grants as well as grants already received); Modest; The Norwegian Breast Cancer Society.

\section{C14.6}

myKinMatters intervention: developing an online intervention to support patients in communicating relevant health information to at-risk relatives

L. M. Ballard, A. Fenwick, A. M. Lucassen 
University Medical Center Groningen, Groningen,

Introduction: Genetic testing can reveal implications for individuals as well as their relatives. Though patients understand the importance of sharing information, especially for those with risks of preventable and treatable diseases, many relatives do not receive this in a timely fashion. Interventions which make it clear for patients whom to inform, how to inform, and what information to convey, are urgently needed.

Methods: We employed a Person Based Approach to ensure the intervention was grounded in the experiences and needs of patients attending a genomic medicine service. This involved: a meta-synthesis of previous interventions; synthesizing communication barriers/facilitators; interviewing 27 participants from the UK's 100,000 Genomes Project; and Systems Thinking to map influences on the target behaviour.

Results: We identified several interventions to aid family communication of genetic results, though most were of poor quality, ineffective, and lacked theoretical underpinning. Interview data showed that (a) many participants had not told many or any relatives they were undergoing genomic testing, and (b) that they would like help to communicate their results to relatives. These results, along with barriers, facilitators, and influences, were used to create the intervention content.

Conclusion: myKinMatters could provide a useful additional tool for clinicians to direct patients towards. As genomic medicine becomes ever more widely available, we hope such online interventions will facilitate at least some communication with relatives that otherwise might not happen. Relatives can then make informed decisions about their own testing, surveillance, or preventative options. Future research involves piloting the intervention. Fellowship: Health Education England

L.M. Ballard: None. A. Fenwick: None. A.M. Lucassen: None.

\section{C16 Personalized and predictive medicine}

\section{C16.1}

What if we would turn a diagnostic multi-cancer gene panel into a screening tool?

L. F. Johansson, K. K. van Dijk-Bos, A. H. van der Hout,

A. P. Knopperts, B. Leegte, J. ter Beest, Y. J. Vos,

C. C. van Diemen, K. Kok, I. M. van Langen,

M. A. Swertz, C. Wijmenga, R. K. Weersma, R. J. Sinke,

B. Sikkema-Raddatz, R. H. Sijmons, H. Westers

Netherlands

Introduction: In cancer genome diagnostics analysis of next-generation sequencing (NGS) gene panel data is focused on the subset of genes with a proven association to the phenotype that prompted referral. In this study we investigated which pathogenic variants are detected as secondary findings when genes proven to be associated with other familial cancer types were analyzed as well.

Materials and Methods: We have analyzed NGS panel data of 2,090 patients referred for genome diagnostics for familial cancer. We analyzed SNVs, Indels and CNVs in all 73 or 85 genes from the targeted panel for all patients, regardless of the cancer type prompting referral. Also we screened 1,326 people from the general Dutch population for pathogenic variants in the 85 cancer genes of our targeted panel.

Results: In $10.2 \%$ of patients we detected a variant matching their families' cancer phenotype. We detected secondary findings in $3.9 \%$ of patients. In the general population $3.2 \%$ of individuals carried a pathogenic variant in one of the panel genes. Limiting secondary findings to the genes listed by the EGAPP, ACMG and SFMPP societies as targets for screening and reporting $0.3,0.7$ and $1.4 \%$ actionable secondary findings would have been reported, respectively in patients. For the general population these numbers are $0.1,0.4$ and $1.5 \%$.

Conclusion: Over 1 in 30 persons in the Dutch population carries a pathogenic variant in a cancer predisposing gene. This finding gives an estimate on what to expect if screening for those genes would be offered as extension of diagnostic testing.

L.F. Johansson: None. K.K. van Dijk-Bos: None. A.H. van der Hout: None. A.P. Knopperts: None. B. Leegte: None. J. ter Beest: None. Y.J. Vos: None. C.C. van Diemen: None. K. Kok: None. I.M. van Langen: None. M.A. Swertz: None. C. Wijmenga: None. R.K. Weersma: None. R.J. Sinke: None. B. Sikkema-Raddatz: None. R.H. Sijmons: None. H. Westers: None.

C16.2

European Landscape of $\mathrm{CDH1}$ germline mutations: a new tool to understand hereditary diffuse gastric cancer (HDGC)

J. García Peláez ${ }^{1}$, A. Monteiro ${ }^{1}$, L. Sousa ${ }^{1}$, S. Castedo ${ }^{1,2,3}$,

L. Garrido ${ }^{2}$, G. Michils ${ }^{4}$, V. Bours ${ }^{5,6}$, R. de Putter ${ }^{7}$,

L. Golmard ${ }^{8}$, M. Blanluet ${ }^{8}, C$. Colas $^{8}$, P. Benusiglio',

S. Aretz ${ }^{10,11}$, I. Spier ${ }^{10,11}$, R. Hüneburg ${ }^{11,12}$, L. Gieldon ${ }^{13}$,

E. Schröck ${ }^{13}$, E. Holinski-Feder ${ }^{14,15}$, V. Steinke ${ }^{14,15}$,

D. Calistri $^{16}$, G. Tedaldi ${ }^{16}$, G. Nadia-Ranzani ${ }^{17}$,

M. Genuardi ${ }^{18,19}$, C. Silveira ${ }^{20}$, M. Krajc ${ }^{21}$, A. Blatnik ${ }^{21}$, 
S. Novakovic ${ }^{21}$, A. Patiño-García ${ }^{22}$, J. Soto ${ }^{23}$, C. Lázaro ${ }^{24}$, G. Capelláa ${ }^{24}$, J. Brunet-Vidal ${ }^{25}$, J. Balmaña ${ }^{26}$,

E. Dominguez-Garrido ${ }^{27}$, M. Ligtenberg ${ }^{28}$, E. Fewings ${ }^{29}$, R. Fitzgerald ${ }^{30}$, E. Woodward ${ }^{31,32}$, G. Evans ${ }^{33}$, H. Hanson ${ }^{34}$, K. Lagerstedt-Robinson ${ }^{35}$, S. BajalicaLagercrantz $^{35}$, M. Teixeira ${ }^{36}$, N. Hoogerbrugge $e^{28}$, M. Tischkowitz ${ }^{29}$, C. Oliveira ${ }^{1,3}$

${ }^{1}$ IPATIMUP/i3s, Porto, Portugal, ${ }^{2}$ Centro Hospitalar Universitário S. João, Porto, Portugal, ${ }^{3}$ Faculty of Medicine of the University of Porto, Porto, Portugal, ${ }^{4}$ Centre for Human Genetics, KU Leuven, Leuven, Belgium, ${ }^{5}$ Laboratory of Human Genetics, GIGA-Institute, University of Liège, Liège, Belgium, ${ }^{6}$ Center of Genetics, University Hospital (CHU), Liège, Belgium, ${ }^{7}$ Clinical Genetics department, University Hospital of Ghent, Ghent, Belgium, ${ }^{8}$ Genetic Department, Institut Curie, Paris, France, ${ }^{9}$ Département de Génétique, GH Pitié-Salpêtrière, Paris, France, ${ }^{10}$ Institute of Human Genetics, University of Bonn, Bonn, Germany, ${ }^{11}$ National Center for Hereditary Tumor Syndromes, University of Bonn, Bonn, Germany, ${ }^{12}$ Department of Internal Medicine I, University Hospital Bonn, Bonn, Germany, ${ }^{13}$ Institut für Klinische Genetik, Medizinische Fakultät Carl Gustav Carus, Dresden, Technische Universität Dresden, Dresden, Germany, ${ }^{14}$ Medizinische Klinik und Poliklinik IV, Campus Innenstadt, Klinikum der Universität, Munich, Germany, ${ }^{15}$ MGZ - Medizinisch Genetisches Zentrum, Munich, Germany, ${ }^{16}$ Biosciences Laboratory, Istituto Scientifico Romagnolo per lo Studio e la Cura dei Tumori (IRST) IRCCS, Meldola (Forlì-Cesena), Italy, ${ }^{17}$ Department of Biology and Biotechnology "Lazzaro Spallanzani", University of Pavia, Pavia, Italy, ${ }^{18}$ Fondazione Policlinico Universitario "A. Gemelli" IRCCS, UOC di Genetica Medica, Rome, Italy, ${ }^{19}$ Istituto di Medicina Genomica, Università Cattolica del Sacro Cuore, Rome, Italy, ${ }^{20}$ Genomed Diagnósticos de Medicina Molecular, S. A., Lisboa, Portugal, ${ }^{21}$ Institute of Oncology Ljubljana, Department of Molecular Diagnostics, Ljubljana, Slovenia, ${ }^{22}$ Clinical Genetics Unit and CIMA LAB Diagnostics. University Clinic of Navarra, Pamplona, Spain, ${ }^{23}$ Molecular Genetics Laboratory, Elche University Hospital, Elche, Spain, ${ }^{24}$ Hereditary Cancer Program, Catalan Institute of Oncology, IDIBELL and CIBERONC, Barcelona, Spain,

${ }^{25}$ Hereditary Cancer Programme, Catalan Institute of Oncology (ICO), Bellvitge Institute for Biomedical Research (IDIBELL), L'Hospitalet Del Llobregat, Barcelona and Biomedical Research Institute (IDIBGI), Girona, Spain, ${ }^{26}$ Hospital Vall d'Hebron and Universitat Autonoma de Barcelona, Barcelona, Spain, ${ }^{27}$ Molecular Diagnostics Laboratory, Fundación Rioja Salud, Logroño, Spain, ${ }^{28}$ Department of Human Genetics, Radboud University Medical Center and Radboud Institute for Molecular Life Sciences, Nijmegen, Netherlands, ${ }^{29}$ Academic Laboratory of Medical Genetics. National Institute for Health Research Cambridge Biomedical Research Centre, University of Cambridge, Cambridge, United Kingdom, ${ }^{30} \mathrm{MRC}$ Cancer Unit, Hutchison-MRC Research Centre, University of Cambridge, Cambridge, United Kingdom, ${ }^{31} \mathrm{NW}$ Genomic Laboratory hub, Manchester Centre for Genomic Medicine, Manchester University Hospitals NHS Foundation Trust, Manchester, United Kingdom, ${ }^{32}$ Division of Evolution and Genomic Sciences, School of Biological Sciences, Faculty of Biology, Medicine and Health, University of Manchester, Manchester Academic Health Science Centre, Manchester, United Kingdom, ${ }^{33}$ Manchester Centre for Genomic Medicine, Manchester Centre for Genomic Medicine, Manchester, United Kingdom, ${ }^{34}$ St Georges NHS Foundation Trust, London, United Kingdom, ${ }^{35}$ Department of Molecular Medicine and Surgery, Karolinska Institutet and Department of Clinical Genetics, Karolinska Univ Hospital, Stockholm, Sweden, ${ }^{36}$ IPO, Instituto Português de Oncologia do Porto, Porto, Portugal

CDH1 germline mutations/deletions cause Hereditary Diffuse Gastric Cancer-HDGC that predisposes for earlyonset diffuse gastric cancer-DGC and lobular breast cancerLBC. Carriers of Pathogenic/Likely pathogenic-P/LP variants undergo intensive screening and/or risk-reduction gastrectomy/mastectomy. Herein, we explored the phenotypic landscape of a European-cohort of $\mathrm{CDH1}$-mutationcarriers to demonstrate the value of genetic-testing driven by phenotype and clinical-criteria.

We collected and curated variant classification, clinicalcriteria and phenotypes from 700 European $\mathrm{CDH} 1$-mutation-carriers of 10 European Countries belonging to ERNGENTURIS. We excluded those presenting $3^{\prime}$-UTR, 5'UTR, intronic variants outside splicing-regions and unclassifiable to date. We focused on 389 probands bearing 214 different $C D H 1$ variants.

In our cohort, 137 families fulfilled HDGC, 31 had borderline-HDGC-criteria, 221 lacked clinical criteria. While $85 \%$ of $\mathrm{HDGC} /$ borderline-HDGC cases carried largerearrangements or truncating variants, $90 \%$ of those lacking criteria carried missense mutations. Based on ACMG classification, 155 families carried P/LP actionable-variants: 76\% HDGC; $15 \%$ borderline-HDGC, and 10\% lacking criteria. Large-rearrangements occurred in $20 \%$ of HDGC/ borderline-HDGC families. Families bearing rearrangements either presented DGC only or LBC only, while families bearing point-mutations more often displayed DGC only. The most common CDH1 germline mutation in Europe is Del_Ex1-2 found in 18 families from 6 different countries. Twenty P/LP variants are recurrent in 90 families across Europe.

This is the first family-driven analysis performed in a curated-cohort of $\mathrm{CDH}$-mutation-carriers. It demonstrates 
the value of genetic-testing driven by phenotype and clinical-criteria, highlighting rearrangements as genetic cause in $20 \%$ of families fulfilling/borderline HDGC, one of which is the most frequent in European HDGC families.

Funding: SolveRD/H2020-Grant/Ref. 779257; ERNGENTURIS; Portuguese FCT/FEDER-COMPETE-POCI01-0145-FEDER-030164

J. García Peláez: None. A. Monteiro: None. L. Sousa: None. S. Castedo: None. L. Garrido: None. G. Michils: None. V. Bours: None. R. de Putter: None. L. Golmard: None. M. Blanluet: None. C. Colas: None. P. Benusiglio: None. S. Aretz: None. I. Spier: None. R. Hüneburg: None. L. Gieldon: None. E. Schröck: None. E. HolinskiFeder: None. V. Steinke: None. D. Calistri: None. G. Tedaldi: None. G. Nadia-Ranzani: None. M. Genuardi: None. C. Silveira: None. M. Krajc: None. A. Blatnik: None. S. Novakovic: None. A. Patiño-García: None. J. Soto: None. C. Lázaro: None. G. Capellá: None. J. Brunet-Vidal: None. J. Balmaña: None. E. DomínguezGarrido: None. M. Ligtenberg: None. E. Fewings: None. R. Fitzgerald: None. E. Woodward: None. G. Evans: None. H. Hanson: None. K. Lagerstedt-Robinson: None. S. Bajalica-Lagercrantz: None. M. Teixeira: None. N. Hoogerbrugge: None. M. Tischkowitz: None. C. Oliveira: None.

\section{C16.3}

Clinical applicability of the 313-SNP based polygenic risk score for breast cancer risk prediction

\section{M. M. Lakeman ${ }^{1}$, M. Rodriguez-Girondo ${ }^{1}$, A. Lee ${ }^{2}$, A. Hollestelle ${ }^{3}$, M. K. Schmidt ${ }^{4}$ C. J. van Asperen ${ }^{1}$, P. Devilee ${ }^{1}$, HEBON consortium}

${ }^{1}$ Leiden University Medical Center, Leiden, Netherlands, ${ }^{2}$ University of Cambridge, Cambridge, United Kingdom, ${ }^{3}$ Erasmus MC Cancer Institute, Rotterdam, Netherlands, ${ }^{4}$ Netherlands Cancer Institute, Amsterdam, Netherlands

Introduction: Breast cancer associated SNPs are presently not used to guide clinical management. We explored whether a genetic test that incorporates a 313-SNP-based Polygenic Risk Score (PRS) is clinically meaningful in nonBRCA1/2 Dutch breast cancer families.

Materials and Methods: We included 2186 breast cancer cases from 1907 Dutch non-BRCA1/2 breast cancer families. The 313-SNP PRS was calculated and standardised to 3479 Dutch population controls (sPRS). The association of the sPRS with breast cancer was estimated using a logistic regression model, adjusted for family history. Standard errors were corrected to account for relatedness of individuals. Using the recently extended BOADICEA model, with and without individual PRS, lifetime risks were retrospectively calculated by simulating an individual to be at an age of one year and unaffected (for cases), i.e., lifetime risk at birth, given the family history.

Results: The mean sPRS for breast cancer cases was 0.65 $(\mathrm{SD}=0.88,95 \% \mathrm{CI}=[0.62-0.69])$. We found a significant association between sPRS and breast cancer, $\mathrm{OR}=1.70$, 95\% CI[1.67-1.72], p-value $<2$ e-16. Including the sPRS to BOADICEA family based risk prediction would have changed screening recommendations in 26,33 , and $36 \%$ of the cases according to breast cancer screening guidelines from the USA, United Kingdom and the Netherlands (NCCN, NICE, and IKNL), respectively. For the population controls, lacking family data, this was 5, 12 and 5\% respectively.

Conclusion: Our results confirm the association between breast cancer and the 313-SNP PRS in the Dutch population and support the application of the PRS in risk prediction for genetically unexplained breast cancer families.

I.M.M. Lakeman: None. M. Rodriguez-Girondo: None. A. Lee: None. A. Hollestelle: None. M.K. Schmidt: None. C.J. van Asperen: None. P. Devilee: None.

\section{C16.4}

High polygenic risk contributes to an early disease onset in common cardiometabolic diseases and cancers

N. J. Mars ${ }^{1}$, J. Koskela ${ }^{1}$, P. Ripatti ${ }^{1}$, T. Kiiskinen ${ }^{1}$, A. S. Havulinna ${ }^{1,2}$, L. Groop ${ }^{1,3}$, A. Palotie ${ }^{1,4,5}$, M. Daly ${ }^{1,4,5}$, V. Salomaa ${ }^{2}$, E. Widén ${ }^{1}$ S. Ripatti, ${ }^{1,6,7}$

${ }^{1}$ Institute for Molecular Medicine Finland, HiLIFE, University of Helsinki, Helsinki, Finland, ${ }^{2}$ National Institute for Health and Welfare, Helsinki, Finland, ${ }^{3}$ Diabetes and Endocrinology, Lund University Diabetes Centre, Malmö, Sweden, ${ }^{4}$ Analytic and Translational Genetics Unit, Massachusetts General Hospital, Boston, MA, United States, ${ }^{5}$ Stanley Center for Psychiatric Research, Broad Institute of MIT and Harvard, Cambridge, MA, United States, ${ }^{6}$ Broad Institute of MIT and Harvard, Cambridge, MA, United States, ${ }^{7}$ Department of Public Health, University of Helsinki, Helsinki, Finland

While polygenic risk scores (PRS) have shown promise in risk stratification in common diseases, evidence about their ability to predict future cases alongside clinical risk factors is scarce. Leveraging up to 23 years of prospective data from the population-based FINRISK study $(\mathrm{n}=21,951)$, we built genome-wide PRSs with $>6$ million variants for coronary heart disease (CHD), atrial fibrillation (AF), type 2 diabetes (T2D), breast cancer, and prostate cancer and tested their 1) associations with incident cases, 2) impact on age at disease onset, and 3) impact in prediction alongside established clinical risk factors. 
A high PRS $\left(>97.5^{\text {th }}\right.$ percentile) was strongly associated with higher disease incidence (hazard ratios 3.73-38.29 for top vs bottom $2.5 \%$, p-values $0.0003-6.65 \times 10^{-22}$ ). This risk increase translated into earlier disease onset by 9 to 29 years in the high-risk group. For example, for T2D the age at onset in the high-risk group was 29.0 years earlier than in the low-risk group.

Of 297 early-onset CHD (age <55) cases, the clinical ASCVD risk calculator for cardiovascular diseases identified only $13.1 \%$ as high risk $(\geq 7.5 \%)$; high $\left(>80^{\text {th }}\right.$ percentile) CHD PRS was the only risk factor in $31.0 \%$ and a familial hypercholesterolemia mutation was present in $0.3 \%$. Excluding age as a risk factor, the PRS had a higher effect for CHD risk than did the routinely measured clinical risk factors combined, with similar findings for $\mathrm{AF}$ and T2D.

In summary, high polygenic risk predicts future disease and is strongly associated with premature disease onset.

N.J. Mars: None. J. Koskela: None. P. Ripatti: None. T. Kiiskinen: None. A.S. Havulinna: None. L. Groop: None. A. Palotie: None. M. Daly: None. V. Salomaa: None. E. Widén: None. S. Ripatti: None.

\section{C16.5}

Clinically actionable results from a multi-gene screening panel in an unselected "healthy" Canadian population

\section{H. J. Andrighetti ${ }^{1}$, J. Y. J. Gu ${ }^{1}$, A. Hazell, J. Furnival, M. Zarb ${ }^{1}$ L. Velsher ${ }^{1,2}$ \\ ${ }^{1}$ Medcan, Toronto, ON, Canada, ${ }^{2}$ North York General Hospital, Toronto, ON, Canada}

Introduction: Integrating genomic screening into primary care may help individualize medical management, ideally leading to early disease detection and reduced mortality. Data are limited regarding prevalence and penetrance of "rare" actionable genetic conditions in the unselected ("healthy") population. We aim to quantify the prevalence of clinically actionable variants in a healthy Canadian population.

Methods: Since September 2017, patients of Medcan, a preventive wellness clinic in Toronto, may opt in (self-pay) to Invitae's proactive screening panel of $130+$ genes, with pre- and post-test genetic counselling. Gene list is derived from ACMG59 recommendations with additional clinically actionable genes, primarily hereditary cancer and cardiac predispositions. Those positive for pathogenic or likely pathogenic variants are referred for additional screening and management per existing clinical guidelines.

Results: Data collection is ongoing, anticipating 1700+ patients by June 2019. Data from our first 1300 patients reveal a positive rate of $14.2 \%$, excluding carriers of recessive conditions. Removing biallelic hemochromatosis variants, the positive rate is $9.8 \%$. Limiting to those reportable per ACMG59 criteria (i.e. excluding $M U T Y H$ heterozygotes, $A P C$ I1307K carriers, and variants in nonACMG59 genes, like CHEK2 and PALB2), positive rate is $3.7 \%$. Of all positives, only $5.5 \%$ would have qualified for publicly-funded genetic testing.

Conclusion: A substantial rate of clinically actionable genetic findings is seen in a "healthy" Canadian population who would not otherwise access genetic testing. Longitudinal data are needed to determine whether proactive genomic screening leads to improved health outcomes, and whether the cost-benefit balance warrants consideration of population screening within the public healthcare system.

H.J. Andrighetti: A. Employment (full or part-time); Significant; Medcan. J.Y.J. Gu: A. Employment (full or part-time); Significant; Medcan. A. Hazell: A. Employment (full or part-time); Significant; Medcan. J. Furnival: A. Employment (full or part-time); Significant; Medcan. M. Zarb: A. Employment (full or part-time); Significant; Medcan. L. Velsher: A. Employment (full or part-time); Significant; Medcan.

\section{C16.6}

Population genomic screening of all young adults in a health-care system: a cost-effectiveness analysis

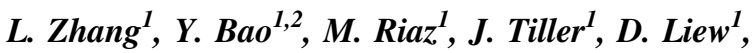 X. Zhuang ${ }^{2}$, D. J. Amor ${ }^{3}$, A. Huq ${ }^{4}$, L. Petelin ${ }^{5}$, M. Nelson ${ }^{6}$, P. J. James ${ }^{7}$, I. Winship ${ }^{7}$, J. J. McNeil', P. Lacaze L $^{1}$

${ }^{1}$ Department of Epidemiology and Preventive Medicine, School of Public Health and Preventive Medicine, Monash University, Melbourne, Australia, ${ }^{2}$ School of Public Health, Nantong University, Nantong, China, ${ }^{3}$ Victorian Clinical Genetics Services; Murdoch Children's Research Institute; Department of Paediatrics, University of Melbourne; Royal Children's Hospital, Parkville, Melbourne, Australia, ${ }^{4}$ epartment of Genomic Medicine, Royal Melbourne Hospital; Department of Medicine, Royal Melbourne Hospital, University of Melbourne, Melbourne, Australia, ${ }^{5}$ Familial Cancer Centre, Peter MacCallum Cancer Centre, Melbourne, Australia, ${ }^{6}$ Discipline of General Practice, University of Tasmania, Hobart, Australia, ${ }^{7}$ Department of Genomic Medicine, Royal Melbourne Hospital; Department of Medicine, Royal Melbourne Hospital, University of Melbourne, Melbourne, Australia

Background: Genomic screening of the adult population has significant potential to prevent disease. Some singlepayer or state-funded healthcare systems are already considering offering population genomic testing to all 
members. Yet the necessary cost-effectiveness analysis of population screening for multiple genetic conditions concurrently has been lacking.

Aim: To forecast the combined impact and costeffectiveness of offering population genomic screening to all young adults in a single-payer healthcare system.

Methods: We modelled screening of 2,688,192 individuals, all adults aged 18-25yo in Australia, for pathogenic variants in BRCA1/BRCA2/MLH1/MSH2 genes, and concurrent preconception carrier screening for cystic fibrosis (CF), spinal muscular atrophy (SMA) and fragile X syndrome (FXS), at $71 \%$ testing uptake, using per-test costs ranging AUD\$200-\$1,200 ( €125-\$760). Investment costs included genetic counseling, surveillance and interventions (reimbursed only) for at-risk individuals/couples. Costeffectiveness was defined below AUD\$50,000/DALY (Disability-Adjusted Life Year) prevented, using an incremental cost-effectiveness ratio (ICER), compared with targeted testing. Outcomes were cancer incidence/mortality, disease cases, and treatment costs reduced.

Results: Population screening would reduce mutationattributable cancers by $28.8 \%$, cancer deaths by $31.2 \%$, and CF/SMA/FXS cases by $24.8 \%$, compared with targeted testing. Assuming AUD\$400 per-test ( €250), investment would be between 4-5 times higher than current expenditure. However, screening would lead to substantial savings in medical costs and DALYs prevented, at a highly costeffective ICER (AUD\$4,038/DALY). At AUD\$200 pertest, screening would approach cost-saving for the health system (ICER = AUD\$22/DALY).

Conclusion: Preventive genomic screening in early adulthood would be highly cost-effective, potentially costsaving, in a single-payer healthcare system. But a range of ethical and service-related issues exist.

L. Zhang: None. Y. Bao: None. M. Riaz: None. J. Tiller: None. D. Liew: None. X. Zhuang: None. D.J. Amor: None. A. Huq: None. L. Petelin: None. M. Nelson: None. P.J. James: None. I. Winship: None. J.J. McNeil: None. P. Lacaze: None.

\section{C17 Genetic mechanisms in cancer}

\section{C17.1}

Identification of IncRNA-mRNA network(s) that modulate prognosis in hepatocellular carcinoma patients

C. G. Lee $e^{1,2,3}$, L. J. Lim ${ }^{1}, J . Y^{2}$, H. C. Toh ${ }^{2}$, P. K. H. Chow $^{4}$, A. Y. F. Chung ${ }^{4}$, L. L. P. J. Ooi ${ }^{4}$, S. S. Chong ${ }^{1}$

${ }^{1}$ National University of Singapore, Singapore, Singapore,

${ }^{2}$ National Cancer Centre, Singapore, Singapore,
${ }^{3}$ Duke-NUS Medical School, Singapore, Singapore, ${ }^{4}$ Singapore General Hospital, Singapore, Singapore

Introduction: Being the 6th most common and 2nd most fatal cancer in the world, prognosis for hepatocellular carcinoma (HCC) patients is poor. It is thus important to better understand its molecular pathogenesis to identify actionable targets. Long non-coding RNAs (LncRNAs) have recently been shown to be de-regulated in various cancers, including HCC. We hypothesize that lncRNAs can serve as master regulators to de-regulate gene expression in cancer pathways to modulate the prognosis of $\mathrm{HCC}$ patients.

Materials and Methods: The profile of lncRNAs and mRNAs were determined in the tumor and adjacent nontumorous tissues of HCC patients. Pearson Correlation analysis was employed to identify mRNAs that are strongly correlated with lncRNAs. These lncRNAs and their correlated mRNAs were then evaluated for association with clinical phenotypes (e.g. tumor grade). IncRNA-mRNA coexpression network analyses was then performed to identify oncogenic or tumor suppressive Potential Master IncRNA regulator(s) (PMR) which is significantly correlated with the most mRNAs which share similar clinical phenotype as the PMR.

Results: We identified a total of 37 co-expression networks with 453 clinically associated lncRNA-mRNA pairs. A notable network comprises 5 upregulated PMR lncRNAs with 91 correlated genes which are significantly associated with tumor grade. Genes in this network are enriched in cell-cycle function. Another significant network comprises 2 downregulated lncRNAs significantly correlated with 8 mainly metallothionein protein family genes and are significantly associated with tumor invasion.

Conclusion: These PMR lncRNAs thus have potential to serve as actionable targets for therapeutic intervention.

C.G. Lee: None. L.J. Lim: None. J. Yu: None. H.C. Toh: None. P.K.H. Chow: None. A.Y.F. Chung: None. L. L.P.J. Ooi: None. S.S. Chong: None.

C17.2

Germline $D G C R 8$ p.E518K alters miRNA profiles and predisposes to thyroid goiter and schwannomatosis

B. Rivera ${ }^{I}$, J. Nadaf ${ }^{1}$, M. Apellaniz-Ruiz ${ }^{I}$, S. Fahiminiya ${ }^{I}$, A. Saskin', A. Chong', S. Sharma', R. Wagener ${ }^{2}$, T. Revil ${ }^{1}$, Z. Harra ${ }^{1}$, N. Hamel', N. Sabbaghian', K. Muchantef ${ }^{1}$, C. Thomas ${ }^{1}$, M. Hébert-Blouin', A. Bassenden $^{1}$, O. Mete ${ }^{3}$, R. Paschke ${ }^{4}$, M. Pusztaszeri ${ }^{1}$, W. Paulus ${ }^{5}$, A. Berghuis ${ }^{1}$, J. Ragoussis ${ }^{1}$, R. Siebert ${ }^{2}$, S. Albrecht ${ }^{1}$, R. Turcotte ${ }^{I}$, M. Hasselblatt ${ }^{2}$, M. Fabian ${ }^{1}$, W. Foulkes ${ }^{I}$ 
${ }^{1}$ McGill University, Montreal, QC, Canada, ${ }^{2}$ Ulm University, Ulm, Germany, ${ }^{3}$ University of Toronto, Toronto, $O N$, Canada, ${ }^{4}$ University of Calgary, Calgary, AB, Canada, ${ }^{5}$ Munster University, Munster, Germany

Micro-(mi)RNA biogenesis utilizes DROSHA and DGCR8, which cleave primary miRNA transcripts into miRNA precursors, as well as DICER1, which processes precursors into mature miRNAs. DICER1 is the only known miRNA biogenesis component associated with an inherited tumor syndrome, which features multinodular goiter (MNG) and rare pediatric-onset lesions, including Wilms tumor (WT). Whole exome sequencing in six affected and three nonaffected individuals from a kindred with early-onset MNG identified a germline missense variant in DGCR8, c. $1552 \mathrm{G}>\mathrm{A}$;p.E518K, perfectly segregating with the disease. Five of the six affected individuals also have schwannomatosis and one developed a choroid plexus papilloma (CPP). The variant has never been described in the germline, it is a recurrent somatic mutation in WT and has been reported in differentiated thyroid cancer (DTC). We performed a genome wide screen for copy number alterations in the schwannomas, MNG, DTC, Wilms tumor and CPP tumors heterozygous for p.E518K, and found a copy number loss of Chrom22q, leading to loss of heterozygosity at the p.E518K locus in all samples. DGCR8 lies on chromosome 22q, immediately adjacent to the three best known schwannoma genes: LZTR1, SMARCB1 and NF2. DGCR8-c.1552G>A;p.E518K. MicroRNA and RNA sequencing show that this variant disrupts pri-miRNA to pre-miRNA processing and impacts levels of canonical and non-canonical miRNAs, leading to overexpression of effectors involved in schwannoma development. In summary, we identified $D G C R 8$ as the cause of what appears to be an autosomal dominant mendelian tumor susceptibility syndrome: familial MNG with schwannomatosis. Funded: CIHR FDN-148390 (WDF). The ALSF and the Mia-Neri Foundation (BR)

B. Rivera: None. J. Nadaf: None. M. Apellaniz-Ruiz: None. S. Fahiminiya: None. A. Saskin: None. A. Chong: None. S. Sharma: None. R. Wagener: None. T. Revil: None. Z. Harra: None. N. Hamel: None. N. Sabbaghian: None. K. Muchantef: None. C. Thomas: None. M. Hébert-Blouin: None. A. Bassenden: None. O. Mete: None. R. Paschke: None. M. Pusztaszeri: None. W. Paulus: None. A. Berghuis: None. J. Ragoussis: None. R. Siebert: None. S. Albrecht: None. R. Turcotte: None. M. Hasselblatt: None. M. Fabian: None. W. Foulkes: None.

\section{C17.3}

Structural variations at $\mathrm{CDH} 1$ intronic cis-regulatory elements cause $\mathrm{CDH} 1 / \mathrm{E}$-cadherin loss of function
R. Barbosa-Matos ${ }^{1,2}$, P. Oliveira ${ }^{1,2}$, B. Mesquita $^{1,2}$, A. André ${ }^{1,2}$, H. Pinheiro ${ }^{1,2}$, J. Carvalho ${ }^{1,2}$, J. Senz $^{3}$, P. Kaurah ${ }^{4}$, R. Bordeira-Carriço ${ }^{5,2}, J^{\text {B Bessa }}{ }^{5,2}$, D. Huntsman ${ }^{3,6,7}$, A. Ferro ${ }^{1,2}$, C. Oliveira ${ }^{1,2,8}$

${ }^{1}$ IPATIMUP, Institute of Molecular Pathology and Immunology, University of Porto, Porto, Portugal, ${ }^{2}$ i3S, Institute for Investigation and Innovation in Health, University of Porto, Porto, Portugal, ${ }^{3}$ Centre for Translational and Applied Genomics, British Columbia Cancer Agency, Vancouver, BC, Canada, ${ }^{4}$ Department of Medical Genetics, University of British Columbia, Vancouver, BC, Canada, ${ }^{5} I B M C$, Institute for Molecular and Cell Biology, University of Porto, Porto, Portugal, ${ }^{6}$ Genetic Pathology Evaluation Centre, University of British Columbia and Vancouver General Hospital, Vancouver, BC, Canada, ${ }^{7}$ Department of Pathology and Laboratory Medicine, University of British Columbia, Vancouver, BC, Canada, ${ }^{8}$ Department of Pathology, Faculty of Medicine of the University of Porto, Porto, Portugal

Introduction: Genomic structural variations in cis-regulatory elements (CREs) can affect gene regulatory networks leading to disease development. E-Cadherin loss-of-function (LoF) correlates with metastatic spread in most epithelial cancers (somatic), early-onset gastric and breast cancer (germline EOGC/EOBC) and cleft-lip/palate, however the underlying mechanisms are vastly unknown.

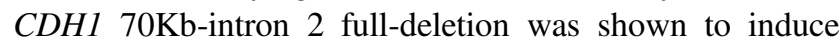
E-cadherin LoF, therefore we hypothesized that structural variation at $\mathrm{CDH} 1$ intronic CREs could further explain $\mathrm{CDH} 1$-associated disease phenotypes.

Materials and Methods: Bioinformatics analysis of genomic/epigenomic regulatory features revealed several $\mathrm{CDH} 1$ CREs, which were sequenced in the germline of EOGC/EOBC patients $(n=250)$. Using CRISPR/Cas9, selected CREs were edited, genotyped and characterized for $\mathrm{CDH1/E-cadherin} \mathrm{expression} \mathrm{in} \mathrm{GC} \mathrm{cell} \mathrm{lines} \mathrm{using} \mathrm{qRT-}$ PCR, allele-specific expression assay, western-blotting and immunocytochemistry. CREs enhancer activity was assessed in zebrafish embryos.

Results: All CREs harbored novel SNVs and/or CNVs in EOGC/EOBC patients. Homozygous editing of two CREs led to $C D H 1$ RNA and protein expression loss in GC cells. CDH1 LoF was caused by inversions and/or deletions affecting CRE sequences. In vivo, both CREs displayed tissue-specific enhancer activity, particularly in the mouth and/or midline of Zebrafish embryos, areas where $\mathrm{CDH} 1$ plays a crucial role during development.

Conclusion: With our combined dry/wet-lab approaches, we narrowed down regions within $\mathrm{CDH} 1$ intronic regions able to control $C D H 1 / \mathrm{E}-\mathrm{Cadherin}$ expression and function. We further unveiled that both deletions and inversions of 
CREs may lead to E-cadherin LoF, suggesting novel mechanisms worth screening in $\mathrm{CDH}$-associated disease phenotypes.

Funding: 1) Solve-RD project received funding from European Union's Horizon2020 research and innovation programme, grant agreement $\mathrm{N}^{\mathrm{o}}$ :779257;2)FEDER/COMPETE, "POCI-01-0145-FEDER-030164".

R. Barbosa-Matos: None. P. Oliveira: None. B. Mesquita: None. A. André: None. H. Pinheiro: None. J. Carvalho: None. J. Senz: None. P. Kaurah: None. R. Bordeira-Carriço: None. J. Bessa: None. D. Huntsman: None. A. Ferro: None. C. Oliveira: None.

\section{C17.4}

Skipping nonsense to maintain function: the paradigm of $B R C A 2$ exon 12

L. Meulemans ${ }^{1}$, R. Mesman ${ }^{2}$, S. M. Caputo $^{3,4}$, S. Krieger ${ }^{1,5,6}$, M. Guillaud-Bataille ${ }^{7}$, V. CauxMoncoutier $^{3,8}$, M. Léone ${ }^{9}$, N. Boutry-Kryza', J. Sokolowska ${ }^{10}$, H. Tubeuf ${ }^{1,11}$, O. Soukarieh ${ }^{1}$, F. Révillion ${ }^{12}$, C. Delnatte ${ }^{13}$, F. Bonnet-Dorion ${ }^{14}$,

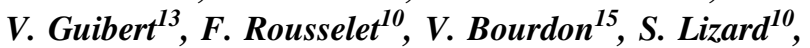
P. Vilquin ${ }^{16}$, C. Grout ${ }^{1}$, A. Drouet ${ }^{1}$, F. M. G. R. Calléja ${ }^{2}$, L. Golmard ${ }^{3}$, H. Vrieling ${ }^{2}$, D. Stoppa-Lyonnet ${ }^{3,8}$, C. Houdayer ${ }^{1,3,17}$, T. Frebourg ${ }^{1,17}$, M. Vreeswijk ${ }^{2}$, A. Martins ${ }^{1}$, P. Gaildrat ${ }^{1}$

${ }^{1}$ Normandie Univ, UNIROUEN, Inserm U1245, Normandy Centre for Genomic and Personalized Medicine, Rouen, France, ${ }^{2}$ Department of Human Genetics, Leiden University Medical Center, Leiden, Netherlands, ${ }^{3}$ Department of Genetics, Institut Curie, Paris, France, ${ }^{4}$ Paris Sciences Lettres Research University, Paris, France, ${ }^{5}$ Laboratory of Cancer Biology and Genetics, Centre François Baclesse, Caen, France, ${ }^{6}$ Normandie University, UNICAEN, Caen, France, ${ }^{7}$ Department of Genetics, Institut Gustave Roussy, Villejuif, France, ${ }^{8}$ Inserm U830, University Paris Descartes, Paris, France, ${ }^{9}$ Hospices Civils de Lyon, Bron, France, ${ }^{10}$ Department of Genetics, Nancy University Hospital, Nancy, France, ${ }^{11}$ Interactive Biosoftware, Rouen, France, ${ }^{12}$ Unit of Human Molecular Oncology, Centre Oscar Lambret, Lille, France, ${ }^{13}$ Department of Genetics, Nantes University Hospital, Nantes, France, ${ }^{14}$ Inserm U916, Department of Genetics, Institut Bergonié, Bordeaux, France, ${ }^{15}$ Department of Genetics, Institut PaoliCalmettes, Marseille, France, ${ }^{16}$ Department of Pathology and Oncobiology, Montpellier University Hospital, Montpellier, France, ${ }^{17}$ Department of Genetics, Rouen University Hospital, Rouen, France

Germline nonsense and canonical splice site variants identified in disease-causing genes are generally considered, unambiguously, as null alleles and classified as pathogenic. However, a fraction of such variants could maintain function through their impact on RNA splicing. To test this hypothesis, we used the alternatively spliced $B R C A 2$ exon 12 (E12) as a model, since its in-frame skipping leads to a potentially functional protein. All BRCA2 E12 variants corresponding to putative null variants or predicted to alter splicing $(\mathrm{n}=39)$ were selected from databases and characterized for their impact on splicing in minigene assays and in patient lymphoblastoid cell lines, when available. Moreover, a selection of variants was analyzed in mouse embryonic stem cell-based functional assays. Using these complementary approaches, we demonstrate that a subset of variants, including nonsense variants, (i) induces in-frame E12 skipping through the modification of splice sites or regulatory elements and, as a consequence, (ii) leads to the production of an internally deleted but partially functional protein. These data provide evidence for the first time in a cancer predisposition gene that variant-induced perturbation of splicing can, at least partially, rescue the loss of function of certain presumed null variants. Additional studies are required to estimate cancer-risk associated with these hypomorphic variants. The existence of such rescue mechanism may question the pathogenic classification of certain null variants identified in disease-causing genes. Our findings emphasize the importance of RNA-based analyses and assessment of protein function to ensure accurate variant classification, a paramount step in genomic medicine.

L. Meulemans: None. R. Mesman: None. S.M. Caputo: None. S. Krieger: None. M. Guillaud-Bataille: None. V. Caux-Moncoutier: None. M. Léone: None. N. BoutryKryza: None. J. Sokolowska: None. H. Tubeuf: None. O. Soukarieh: None. F. Révillion: None. C. Delnatte: None. F. Bonnet-Dorion: None. V. Guibert: None. F. Rousselet: None. V. Bourdon: None. S. Lizard: None. P. Vilquin: None. C. Grout: None. A. Drouet: None. F.M.G.R. Calléja: None. L. Golmard: None. H. Vrieling: None. D. Stoppa-Lyonnet: None. C. Houdayer: None. T. Frebourg: None. M. Vreeswijk: None. A. Martins: None. P. Gaildrat: None.

\section{C17.5}

Genome-wide association study identifies pathways associated with cervical cancer risk

D. Ramachandran', J. Dennis ${ }^{2}$, L. Fachal', P. Schürmann ${ }^{1}$, I. Runnebaum ${ }^{3}$, M. Dürst ${ }^{3}$, P. Hillemanns ${ }^{1}$, D. F. Easton ${ }^{2,4}$, T. Dörk ${ }^{1}$

${ }^{1}$ Gynaecology Research Unit, Hannover Medical School, Hannover, Germany, ${ }^{2}$ University of Cambridge, Centre for Cancer Genetic Epidemiology, Department of Public 
Health and Primary Care, Cambridge, United Kingdom, ${ }^{3}$ Department of Gynecology, Jena University Hospital, Friedrich -Schiller-University Jena, Jena, Germany, ${ }^{4}$ University of Cambridge, Centre for Cancer Genetic Epidemiology, Department of Oncology, Cambridge, United Kingdom

Cervical cancer is triggered by human papillomavirus infection but only a minority of HPV infected women show progress towards invasive disease. Genetic factors and pathways underlying cervical cancer are largely unknown. We performed a genome-wide association study to unravel low-risk cervical cancer susceptibility variants in the German population. A series of 375 patients with invasive cervical cancer and 866 healthy controls were directly genotyped for $\sim 570,000$ variants on the Illumina OncoArray BeadChip. After genotype calling, QC, PCA, and imputation, we used PLINK to analyze 21,326,396 variants for their association with cervical cancer. We did not identify single variants at the level of genome-wide significance. However, a gene-based analysis using MAGMA through mapping SNPs to 19,213 protein-coding genes indicated a significant role for OSGINI ( $\mathrm{p}=1.12 \times 10 \mathrm{E}-8)$ encoding a p53-regulated tumor suppressor. Pathway analyses using MAGMA detected an enrichment for the GO term "Protein kinases" $(p=0.02)$. To look deeper into possible pathways involved, the SNPs were filtered more stringently using ANNOVAR positional mapping, eQTL, 3D chromatin interaction mapping and common features of chromatin regulation, and 180 prioritized genes were tested using GENE2FUNC. We found significant enrichment for genes residing in a breast cancer $12 q$ amplicon $(p=$ $1.9 \times 10 \mathrm{E}-14)$ and for genes that had emerged from a previous GWAS for "Response to platinum-based neoadjuvant chemotherapy in cervical cancer" $(\mathrm{p}=3.6 \mathrm{x} 10 \mathrm{E}-18)$. Taken together, our findings strongly support the hypothesis that genetic factors play a significant role in the etiology of invasive cervical cancer. Funded by the Bruno and Helene Jöster Foundation.

D. Ramachandran: None. J. Dennis: None. L. Fachal: None. P. Schürmann: None. I. Runnebaum: None. M. Dürst: None. P. Hillemanns: None. D.F. Easton: None. T. Dörk: None.

\section{C17.6}

Mitochondrial damage due to a genetic origin explains the autoimmune response that leads to gastric neuroendocrine tumors

\section{O. Calvete ${ }^{1}$, R. Marra ${ }^{1}$, J. Reyes $^{2}$, J. Benitez $^{I}$}

${ }^{1}$ Centro Nacional de Investigaciones Oncologicas (CNIO), Madrid, Spain, ${ }^{2}$ Centro Nacional de Hospital Comarcal de
Inca. Balearic Islands Health Investigation Institute (IDISBA) Oncologicas (CNIO), Inca, Spain

Type I gastric neuroendocrine tumors (gNETs) arise from hypergastrinemia in patients with autoimmune chronic atrophic gastritis. According to the classical model, the gastric $\mathrm{H}+/ \mathrm{K}+\mathrm{ATPase}$ was the causative autoantigen recognized by $\mathrm{CD} 4+\mathrm{T}$ cells in chronic autoimmune scenario that produces the parietal cell atrophy, which correlates with gastric achlorhydria and increases the risk for gNETs and gastric neoplasms. However, the mechanism by which the inflammatory response correlates with IL-17 secretion and PC atrophy is not clearly defined. Recently, we found that the ATP4 $\mathrm{A}^{\text {p.R703C }}$ mutation impaired PC function and normal gastric acidification, which drove familial gNET. Our group constructed a knock-in mouse model for the ATP4A mutation, which at one year recapitulated human premalignant conditions and achlorhydria. We have monitored the mouse model for one more year and have served us to better understand the relation between impaired capability to export protons across the plasma membrane of PCs and tumor progression. The ATP4A $\mathrm{A}^{\mathrm{p} . \mathrm{R} 703 \mathrm{C}}$ mutation drives gastric achlorhydria, but also deregulates the acidbase balance within PCs, affecting mitochondrial biogenesis. Mitochondrial malfunction activates ROS signaling, which triggers caspase-3-mediated apoptosis. Therefore, mitochondria alteration would initiate the chronic inflammation and does not involve an IL-17 secretion-mediated mechanism rather than the classical explanation. In addition, by restoring gastric euchlorhydria, mitochondrial function is recovered. Finally, infection by $H$. pylori promotes destabilization of the mitochondria of the PCs by a mechanism similar to that described for APT4A $\mathrm{A}^{\mathrm{p} . \mathrm{R} 703 \mathrm{C}}$ carriers, which suggests that restoring gastric euchlorhydria might be indicated for the treatment of these patients.

O. Calvete: None. R. Marra: None. J. Reyes: None. J. Benitez: None.

\section{C18 Therapies}

\section{C18.1}

AAVHSC15 Packaging Human Phenylalanine Hydroxylase Results in Sustained in vivo Correction of Phenylketonuria Following a Single IV Administration in the Murine Model

S. S. Ahmed, M. Wang, J. L. Ellsworth, O. L. Francone, D. Faulkner, A. Sengooba, H. Rubin, S. Dollive, D. Lamppu, T. Wright, D. Kinch, A. Seymour

Homology Medicines Inc, Bedford, MA, United States 
Phenylketonuria (PKU) is a rare hepatic metabolic disease caused by mutations in the phenylalanine hydroxylase $(P A H)$ gene causing elevated levels of phenylalanine that lead to neurocognitive defects. Current treatments do not address the underlying genetic defect. A suite of Clade $\mathrm{F}$ adeno-associated viruses was isolated from human peripheral blood CD34+ hematopoietic stem cells (AAVHSCs) and show high hepatic affinity and potential for gene therapy. Here we describe the ability of AAVHSC-mediated gene therapy to durably correct the disease phenotype in the $P a h^{\text {enu2 }}$ mouse model of severe PKU.

Gene therapy vectors containing a human $P A H$ transgene driven by ubiquitous or liver-specific promoters packaged in AAVHSC15 were tested in $P a h^{\text {enu2 }}$ mice. Vectors were administered in a single intravenous dose followed by periodic assessment of serum phenylalanine and tyrosine (byproduct of phenylalanine metabolism). At study termination, livers were processed to measure vector genomes, mRNA and PAH enzyme activity.

One week post-dose of an initial research vector using an ubiquitous promoter, phenylalanine levels decreased from $2000 \mu \mathrm{M}$ to $<150 \mu \mathrm{M}(\mathrm{p}<0.0001)$ and were sustained out to $>28$ weeks $(\mathrm{p}<0.0001)$. To enhance long-term durability with decreased doses, vector sequences in AAVHSC15PAH were optimized using different liver-specific promoters. We selected a vector, HMI-102, that decreased serum phenylalanine and increased serum tyrosine at tenfold lower doses compared to the initial research vector. A durable response was seen out to 48 weeks, consistent with the model's lifespan. These data demonstrate that a single dose of HMI-102 resulted in long-term correction of PKU in $P a h^{\text {enu2 }}$ mice on protein-containing chow.

S.S. Ahmed: A. Employment (full or part-time); Significant; Homology Medicines Inc. M. Wang: A. Employment (full or part-time); Significant; Homology Medicines Inc. J.L. Ellsworth: A. Employment (full or part-time); Significant; Homology Medicines Inc. O.L. Francone: A. Employment (full or part-time); Significant; Homology Medicines Inc. D. Faulkner: A. Employment (full or part-time); Significant; Homology Medicines Inc. A. Sengooba: A. Employment (full or part-time); Significant; Homology Medicines Inc. H. Rubin: A. Employment (full or part-time); Significant; Homology Medicines Inc. S. Dollive: A. Employment (full or part-time); Significant; Homology Medicines Inc. D. Lamppu: A. Employment (full or part-time); Significant; Homology Medicines Inc. T. Wright: A. Employment (full or part-time); Significant; Homology Medicines Inc. D. Kinch: A. Employment (full or part-time); Significant; Homology Medicines Inc. A. Seymour: A. Employment (full or part-time); Significant; Homology Medicines Inc.

\section{C18.2}

Viral vector therapy as a therapeutic option for peripheral nerve disease associated with metachromatic leukodystrophy

\section{S. K. Newman, T. Rupar}

\section{University of Western Ontario, London, ON, Canada}

Metachromatic leukodystrophy (MLD) is an autosomal recessive neurodegenerative disease caused by the deficiency of the lysosomal enzyme arylsulfatase A (ARSA) leading to accumulation of sulfatide, causing degeneration of myelin in the CNS and PNS. Currently, no treatment exists. Hematopoietic stem cell transplantation (HSCT) provides modest benefit in pre-symptomatic patients but this well short of a realistic treatment. Gene transfer experiments using lenti viral vectors have shown success in decreasing sulfatide accumulation in the CNS of $A_{R S A^{-1-}}$ mouse models; however, this treatment has shown no success in targeting the peripheral nervous system. In the present study, we compared single stranded (ss) AAV serotypesAAV-9 and AAVrh10, packaged with a codon-optimized ARSA gene or eGFP. The vectors were delivered via tail vein injection for PNS distribution to $A R S A^{-1-}$ mice at 6 months of age at a dose of $2 \times 10^{10}$ vector grams per mouse. The gene transfer facilitated both GFP bio distribution (measured via PCR and histopathology) and ARSA bio distribution (measured via HPLC and PCR) along with detectable enzymatic activity throughout the sciatic nerve, spinal cord and liver. Histopathological analysis of the spinal cord and sciatic nerve show presence of GFP indicating positive vector transfection, with AAV-9 showing the most evident GFP presence. In summary, we demonstrate for the first time that tail vein injection of AAV-9 and AAVrh10 to an $A R S A^{-/}$mouse as a treatment strategy for MLD is successfully able to target the PNS and significantly decrease sulfatide accumulation in PNS associated tissue which has a direct therapeutic benefit.

S.K. Newman: None. T. Rupar: None.

\section{C18.3}

Safe and efficient personalised TALEN- and CRISPR/ Cas9-based gene correction therapy for $\beta$-thalassaemia by non-viral delivery to primary cells
P. Patsalis ${ }^{1,2}$, G. Turchiano $^{3,4}$, P. Papasavva ${ }^{1,5}$, M. Romito $^{3,4}$, C. Loucari $^{1,5}$, C. Stephanou ${ }^{1,2}$, S. Christou, M. Sitarou ${ }^{6}$, C. Mussolino ${ }^{3,4}$, T. I. Cornu ${ }^{3,4}$, M. N. Antoniou ${ }^{2}$, C. W. Lederer ${ }^{1,5}$, T. Cathomen ${ }^{3,4}$, M. Kleanthous ${ }^{1,5}$ 
${ }^{1}$ Molecular Genetics of Thalassemia Department, The Cyprus Institute of Neurology and Genetics, Nicosia, Cyprus, ${ }^{2}$ Department of Medical and Molecular Genetics, King's College London, London, United Kingdom, ${ }^{3}$ Institute for Transfusion Medicine and Gene Therapy, Medical Center - University of Freiburg, Freiburg, Germany, ${ }^{4}$ Center for Chronic Immunodeficiency, Medical Center University of Freiburg, Freiburg, Germany, ${ }^{5}$ Cyprus School of Molecular Medicine, Nicosia, Cyprus, ${ }^{6}$ Thalassemia Centre, Cyprus Ministry of Health, Nicosia, Cyprus

Introduction: Homology-directed repair dependent gene therapies can suffer from low efficiencies unsuitable for clinical application. This study demonstrates that correction of the common $H B B^{I V S I-110(G>A)} \beta$-thalassemia splice mutation may be achieved by efficient non-homologous end joining-mediated disruption of the aberrant regulatory elements (DARE) created by the mutation.

Material and Methods: DARE correction was evaluated in patient-derived $\mathrm{CD} 34^{+}$cells by nucleofection of in vitro synthesized TALEN mRNAs and of CRISPR/Cas9 RNAguided nuclease (RGN) ribonucleoprotein complexes. Correction efficiency was assessed as restoration of correct splicing at RNA (RT-qPCR), protein (HPLC) and cellmorphology (differential counts) levels, and off-target activity by targeted deep sequencing. Results: Proof-ofprinciple was achieved in patient-derived CD $34^{+}$cells for both nucleases, reaching high bulk disruption efficiencies of up to $90 \%$. Restoration of correct splicing established up to 63 and $77 \%$ of normal $\beta$-globin synthesis for TALENs and RGN, respectively. RGN- off-target activity for the RNF219_AS1 gene was abolished at high on-target correction, by evaluating strategic mismatches and employing the high-fidelity AltR_HiFi_Cas9. Across primary samples, DARE achieved significant correction of the two hallmarks of $\beta$-thalassaemia pathology, $\beta$-globin synthesis and latestage erythroid differentiation.

Conclusion: Our study shows that mutation-specific gene therapy by DARE is highly efficient and holds great potential for many human diseases caused by aberrant regulatory motifs. High level of correction achieved without enrichment, low level of off-targeting and use of virus- and DNA-free delivery in patient-derived $\mathrm{CD} 34^{+}$cells in this study indicate biosafety and efficiency suitable for direct clinical translation.

P. Patsalis: None. G. Turchiano: None. P. Papasavva: None. M. Romito: None. C. Loucari: None. C. Stephanou: None. S. Christou: None. M. Sitarou: None. C. Mussolino: None. T.I. Cornu: None. M.N. Antoniou: None. C.W. Lederer: None. T. Cathomen: None. M. Kleanthous: None.

\section{C18.4}

Therapeutic gene editing for Hutchinson-Gilford progeria syndrome

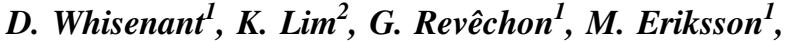 J. S. Kim ${ }^{2}$}

${ }^{1}$ Karolinska Institutet, Huddinge, Stockholm, Sweden, ${ }^{2}$ Institute for Basic Science, Seoul, Korea, Republic of

Hutchinson-Gilford progeria syndrome (HGPS) is a rare premature ageing disorder with clinical manifestations including severe growth retardation, skeletal dysplasia, alopecia, scleroderma-like skin changes, loss of subcutaneous adipose tissue and loss of vascular smooth muscle cells. More than $90 \%$ of the cases are caused by a de novo point mutation in exon 11 of the LMNA gene (LMNA c. 1824 C $>$ T, p.G608G). This mutation activates a cryptic splice site within the LMNA gene and results in a production of a truncated unprocessed lamin A protein called progerin. In this study, we use a conditional humanized mouse model with tissue-specific expression of the common HGPS mutation in keratinocytes of the skin, as well as patientderived cell cultures. For precise single base editing, we are using a CRISPR-Cas9 adenine editor (ABE) in order to reverse the c.1824C $>\mathrm{T}$ point mutation by adeno-associated virus (AAV) delivery. Cell culture experiments with B cellderived lymphoblastoid cells from a HGPS patient show a significant reduction of the c.1824 $\mathrm{C}>\mathrm{T}$ mutation with a correction efficiency up to $76 \%$ and a reduction of the c. $1824 \mathrm{C}>\mathrm{T}$ mutant allele frequency down to $12 \%$ by $\mathrm{ABE}$ treatment. In ongoing experiments we are assessing the AAV delivery system as well as the ABE efficiency in vivo in order to improve the HGPS phenotype. Using this method we have the possibility to target the disease on a genetic level before complex downstream mechanisms are taking place, with the future aim of a highly precise and efficient gene editing approach for clinical use in HGPS patients.

D. Whisenant: None. K. Lim: None. G. Revêchon: None. M. Eriksson: None. J.S. Kim: None.

\section{C18.5}

High efficiency of CRISPR/Cas9 gene editing of T158Mhot spot mutation in $M E C P 2$ gene

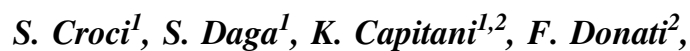
M. L. Carriero ${ }^{1}$, E. Frullanti ${ }^{1}$, V. Lamacchia ${ }^{1,3}$, R. Tita ${ }^{3}$, A. Giliberti ${ }^{1}$, E. Benetti ${ }^{4}$ S. Furini ${ }^{4}$, C. Lo Rizzo ${ }^{3}$, A. M. Pinto $^{3}$, A. Auricchio ${ }^{5}$, S. G. Conticello ${ }^{2}$, A. Renieri $^{1,3}$, I. Meloni $^{1}$ 
${ }^{1}$ Medical Genetics, University of Siena, Siena, Italy, ${ }^{2}$ Molecular Mechanism of Oncogenesis, ITT Core Research Laboratory (CRL), Florence, Italy, ${ }^{3}$ Genetica Medica, Azienda Ospedaliera Universitaria Senese, Siena, Italy, ${ }^{4}$ Department of Medical Biotechnologies, University of Siena, Siena, Italy, ${ }^{5}$ TIGEM (Telethon Institute of Genetics and Medicine), Naples, Italy

Rett syndrome, due to MECP2 mutations, is the second most common cause of intellectual disability in girls. Effective therapies are not yet available. We report here the successful gene editing of one of the most common hot spot mutations, c.473C>T-p.T158M, using CRISPR/Cas9 in patient-derived cells. A two-plasmid system was developed suitable for AAV delivery. The Reporter plasmid expresses the mutation-specific sgRNA and the Donor DNA, which contains the template for the correction. The Cas9 plasmid encodes for the Cas9 flanked by two targets for selfcleavage of the Cas9 coding sequence, in order to avoid long-term expression. We then selected 4 unrelated patients with the same de novo hot spot mutation. Starting from patient-derived fibroblasts, we obtained induced Pluripotent Stem Cells (iPSCs) and iPSC-derived neuronal precursors and neurons. NGS analysis of FACS-isolated cells from three independent patients demonstrated an exceptionally high editing efficiency, ranging from 60 to $80 \%$ of HDR with a very low rate $(1 \%)$ of insertions/deletions. Furthermore, a good AAV9 co-infection was demonstrated in neurons differentiated from iPSCs. To further validate efficacy and safety, in vivo experiments on the Mecp2T158M mouse are ongoing. The described approach is patented at University of Siena and ISPRO (application n. 102018000020230).

S. Croci: None. S. Daga: None. K. Capitani: None. F. Donati: None. M.L. Carriero: None. E. Frullanti: None. V. Lamacchia: None. R. Tita: None. A. Giliberti: None. E. Benetti: None. S. Furini: None. C. Lo Rizzo: None. A. M. Pinto: None. A. Auricchio: None. S.G. Conticello: None. A. Renieri: None. I. Meloni: None.

\section{C18.6}

An open-label, phase 1/2 study of miransertib (ARQ 092), an oral pan-AKT inhibitor, in patients (pts) with PIK3CA-related Overgrowth Spectrum (PROS) and Proteus Syndrome (PS): study design and preliminary results(NCT03094832)

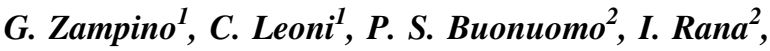 \\ R. Onesimo ${ }^{1}$, M. Macchiaiolo ${ }^{2}$, A. Diociaiuti $^{2}$, \\ S. Livadiotti ${ }^{2}$, N. Resta ${ }^{3}$, Y. Sheldon ${ }^{4}$, R. Savage ${ }^{5}$, \\ M. Lamar $^{5}$, K. Tith ${ }^{5}$ J. Kazakin ${ }^{5}$, B. Schwartz5, \\ D. M. Adams ${ }^{4}$, A. Bartuli
}

${ }^{I}$ Fondazione Policlinico Universitario A. Gemelli, IRCCS, Università Cattolica del Sacro Cuore, Rome, Italy, ${ }^{2}$ Bambino Gesù Children's Hospital, IRCCS, Rome, Italy, ${ }^{3}$ Division of Medical Genetics, Department of Biomedical Sciences and Human Oncology, University of Bari "Aldo Moro”, Bari, Italy, "Vascular Anomalies Center, Boston Children's Hospital, Boston, MA, United States, ${ }^{5}$ ArQule, Inc., Burlington, MA, United States

Introduction: The PI3K/AKT signaling pathway is constitutively active in a number of human malignancies and overgrowth syndromes, including PROS and PS. ARQ 092 is an oral, allosteric, selective pan-AKT inhibitor that potently inhibits both active and inactive forms of AKT. It is currently being evaluated in oncology and overgrowth syndromes clinical trials.

Materials and Methods: Safety, tolerability and preliminary efficacy of ARQ 092 have been tested in 15 pts (8 $\mathrm{F}$ and $7 \mathrm{M}$; age range 2.6-41.1), 14 with $P I K 3 C A$ and 1 with AKT1 mutations. Assessments include physical examination, vital signs, Karnofsky/Lansky performance scale (K/LPS), laboratory tests imaging, pain and patientreported outcomes. All pts receive ARQ 092 at a dose of 15 $\mathrm{mg} / \mathrm{m}^{2} \mathrm{QD}$ with an option to increase to $25 \mathrm{mg} / \mathrm{m}^{2}$. All 15 pts are ongoing and have been on treatment between 3 and 22 months.

Results: Treatment-emergent adverse events (AE) were observed in 15 pts $(100 \%)$, all were grade $\leq 2$. Drug-related AEs were reported in 2 pts: hyperinsulinemia (1 pt); stomatitis and neutropenia (1 pt). The most common AEs were pyrexia $(60 \%)$; cough $(47 \%)$; abdominal pain $(33 \%)$; diarrhea, vomiting (each, 27\%); upper respiratory infection and rhinitis (each, 20\%). No pts were discontinued or dose reduced due toxicity. Radiologically-confirmed stability of lesions and significant improvement in K/LPS, movement fluidity, fatigability and pain relief were noted by parents and clinicians in most pts.

Conclusion: ARQ092 has demonstrated manageable toxicity and preliminary efficacy in PROS/PS pts. Description of the pivotal study design, updated safety and efficacy data will be presented.

G. Zampino: None. C. Leoni: None. P.S. Buonuomo: None. I. Rana: None. R. Onesimo: None. M. Macchiaiolo: None. A. Diociaiuti: None. S. Livadiotti: None. N. Resta: None. Y. Sheldon: None. R. Savage: A. Employment (full or part-time); Modest; ArQule. M. Lamar: A. Employment (full or part-time); Modest; ArQule. K. Tith: A. Employment (full or part-time); Modest; ArQule. J. Kazakin: A. Employment (full or parttime); Modest; ArQule. B. Schwartz5: A. Employment (full or part-time); Significant; ArQule. D.M. Adams: None. A. Bartuli: None. 


\section{C19 From genome architecture to RNA biology}

\section{C19.1}

Phasing of complex genomic rearrangements reveal involvement of both homologous chromosomes in preand post-zigotic events

C. M. Carvalho, C. Beck, Z. Akdemir, F. Sedlazeck, Q. Meng, J. Hu, H. Doddapaneni, E. Chen, S. Jhangiani, A. English, D. Muzni, R. Gibbs, C. Shaw, P. Hastings, J. R. Lupski

Baylor College of Medicine, Houston, TX, United States

Introduction: Complex genomic rearrangements (CGRs) are defined as structural variants consisting of two or more breakpoint junctions and can contribute to as much as $30 \%$ of pathogenic copy number variants (CNVs) in genomic disorders. CGRs are hypothesized to result of an error prone mechanism during repair of a single ended, double stranded DNA that utilizes a poorly processive DNA polymerase resulting in multiple fork collapses and template switching (TS). Majority of CGRs studied show TS within the same chromosome.

Objectives: to gain mechanistic insights into the formation of CGRs.

Material and Methods: we studied six trios on which probands were referred with congenital malformations carrying de novo autosomal CGRs. Trios were studied by combined array comparative genomic hybridization (aCGH), SNP array, targeted $7 \mathrm{Mb}$ Illumina short read, PacBio or Nanopore long-read sequencing.

Results: This combined approach enabled to resolve breakpoint junctions in $6 / 8$ cases. Phasing was obtained by $\mathrm{B}$-allele frequency analysis within the $\mathrm{CNV}$ provided by the trio joint-calling. Surprisingly, in each of the cases here, the amplified segments are constituted by a mix of copies from both homologues rather than originated from only one ancestral chromosome. Two informative cases indicated that the CGRs were formed pre-zygotically, whereas one case was formed post-zygotic.

Conclusion: CNV segments originated from two distinct chromosomes contributing to formation of the same rearrangement strongly support a TS model of generating CGRs. The results shown here predict that CGR are formed by replication-based mechanisms, and can have further implications for sporadic disease, cancer and human gene evolution.

C.M. Carvalho: None. C. Beck: None. Z. Akdemir: None. F. Sedlazeck: None. Q. Meng: None. J. Hu: None. H. Doddapaneni: None. E. Chen: None. S. Jhangiani: None. A. English: None. D. Muzni: None. R. Gibbs:
None. C. Shaw: None. P. Hastings: None. J.R. Lupski: None.

\section{C19.2}

Cytogenetically detected chromosomal inversions are rarely formed by ectopic recombination between inverted repeats

M. Pettersson ${ }^{1}$, C. M. Grochowski ${ }^{2}$, J. Eisfeldt ${ }^{1,3}$, J. Wincent ${ }^{\text {, J. J. R. Lupski }}{ }^{2,4}$, J. Ottosson ${ }^{5}$, L. Lovmar ${ }^{5}$, J. Gacic $^{6}$, E. Syk Lundberg, ${ }^{1,7}$ D. Nilsson ${ }^{1,3,7}$, C. M. B. Carvalho $^{2}$, A. Lindstrand ${ }^{1,7}$

${ }^{1}$ Karolinska Institutet, Stockholm, Sweden, ${ }^{2}$ Baylor College of Medicine, Houston, TX, United States, ${ }^{3}$ Science for Life Laboratory, Stockholm, Sweden, ${ }^{4}$ Texas Children's Hospital, Houston, TX, United States, ${ }^{5}$ Sahlgrenska University Hospital, Gothenburg, Sweden, ${ }^{6}$ Linköping University Hospital, Linköping, Sweden, ${ }^{7}$ Karolinska University Hospital, Stockholm, Sweden

Cytogenetically detected chromosomal inversions are generally assumed to be copy number and phenotypically neutral events. Early cytogenetic studies of inversions suggested that non-allelic homologous recombination (NAHR) between inverted repeats could be the underlying mechanism of formation, and the prevalence of NAHRmediated inversions has been estimated to $67 \%$.

Here we used short-read whole-genome sequencing (WGS) on 16 unique, cytogenetically detected chromosomal inversions and were able to characterize 10/16 (62.5\%) on the nucleotide level. We found that two seemingly recurrent inversions were identical by descent and followup analysis confirmed that the unrelated carriers shared both common and more rare haplotypes on these chromosomes. Out of the 10 inversions that we characterized in detail, nine showed little to no microhomology in the breakpoint junctions, similar to what is commonly seen in reciprocal translocations. Only one inversion was mediated by repeats (Alu-Alu), and had duplications that had been formed concomitantly to the inversion, also through Alu-Alu interactions. Finally, the gene disruption frequency was similar to the frequency obtained for balanced translocations $(6 / 25$ breakpoints, 24\%). In summary, the study indicates that short-read WGS can detect a substantial fraction of chromosomal inversions and resolves the breakpoints on the nucleotide level. Finally, NAHR is likely not the major mechanism underlying the formation of non-recurrent large chromosomal inversions.

Grants: The Royal Physiogtaphic Society in Lund (Nilsson-Ehle donations), Karolinska Institutet funding for doctoral education, SciLifeLab national sequencing 
grant, the Swedish Research Council, the Stockholm County Council, the Ulf Lundahl memory fund through the Swedish Brain Foundation

M. Pettersson: None. C.M. Grochowski: None. J. Eisfeldt: None. J. Wincent: None. J.R. Lupski: None. J. Ottosson: None. L. Lovmar: None. J. Gacic: None. E. Syk Lundberg: None. D. Nilsson: None. C.M.B. Carvalho: None. A. Lindstrand: None.

\section{C19.3}

Optical mapping of 22q11.2 low copy repeats reveals structural hypervariability

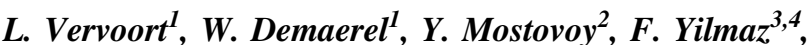
S. Pastor ${ }^{5,6}$, M. Hestand ${ }^{7,8}$, A. Swillen ${ }^{1}$, E. Vergaelen $^{1}$, E. A. Geiger ${ }^{4}$, C. R. Coughlin ${ }^{4}$, S. K. Chow ${ }^{2}$, D. McDonald-McGinn ${ }^{5,6}$, B. E. Morrow ${ }^{9}$, P. Kwok ${ }^{2}$, M. Xiao ${ }^{10}$, B. S. Emmanuel, ${ }^{5,6}$ T. H. Shaikh ${ }^{4}$, J. R. Vermeesch ${ }^{I}$

${ }^{1}$ Department of Human Genetics, KU Leuven, Leuven, Belgium, ${ }^{2}$ Cardiovascular Research Institute, UCSF School of Medicine, San Francisco, CA, United States, ${ }^{3}$ Department of Integrative Biology, University of Colorado Denver, Denver, CO, United States, ${ }^{4}$ Department of Pediatrics, Section of Clinical Genetics and Metabolism, University of Colorado Denver, Aurora, CO, United States, ${ }^{5}$ Division of Human Genetics, Children's Hospital of Philadelphia, Philadelphia, PA, United States, ${ }^{6}$ Department of Pediatrics, Perelman School of Medicine at the University of Pennsylvania, Philadelphia, PA, United States, ${ }^{7}$ Division of Human Genetics, Cincinnati Children's Hospital Medical Center, Cincinnati, OH, United States, ${ }^{8}$ Department of Human Genetics, Cincinnati Children's Hospital Medical Center, Cincinnati, OH, United States, ${ }^{9}$ Department of Genetics, Albert Einstein College of Medicine, Bronx, NY, United States, ${ }^{10}$ School of Biomedical Engineering, Drexel University, Philadelphia, PA, United States

Introduction: The 22q11.2 locus is structurally one of the most complex areas of the human genome due to the presence of low copy repeats (LCR22s). Despite the newest sequencing technologies, the human reference genome hg38 still comprises three unresolved sequence gaps in LCR22-A. The recurrent deletion/duplication breakpoints of 22q11.2 deletion syndrome (22q11.2DS) are embedded within these repeats, but the exact location remains unclarified.

Materials and Methods: We performed an LCR22 de novo assembly using fiber-FISH. Long DNA molecules were extracted from cells, stretched onto coverslips, and hybridized with LCR22 specific probes. Following manual signal screening, alleles were de novo assembled by tiling fibers based on matching colors and distances between the probes.

Results: LCR22 haplotyping in 24 individuals uncovered the presence of 26 different alleles for LCR22-A. These alleles range in size between $200 \mathrm{~kb}$ and $2 \mathrm{Mb}$. Subunits cluster in larger substructures, which vary in orientation, copy number, and presence. In addition, the rearranged alleles of 22q11.2DS patients were mapped in nine families. Differences in the location of the deletion breakpoints were identified. Findings were validated by Bionano optical mapping.

Conclusion: For the first time, the overall architecture of the 22q11.2 locus was successfully unraveled. The LCR22 hypervariability implicates interindividual gene dosage differences. As a consequence, copy number variations could influence gene expression profiles. Additionally, mapped rearrangement breakpoints vary among patients. We hypothesize this variability could provide a genetic explanation for some of the phenotypic variability characterizing the 22q11.2DS.

Funding: FWO GOE1117N, Jerome Lejeune project 1665

L. Vervoort: None. W. Demaerel: None. Y. Mostovoy: None. F. Yilmaz: None. S. Pastor: None. M. Hestand: None. A. Swillen: None. E. Vergaelen: None. E.A. Geiger: None. C.R. Coughlin: None. S.K. Chow: None. D. McDonald-McGinn: None. B.E. Morrow: None. P. Kwok: None. M. Xiao: None. B.S. Emmanuel: None. T. H. Shaikh: None. J.R. Vermeesch: None.

\section{C19.4}

Submicroscopic 13q32.1 deletions causing congenital microcoria modify the regulatory landscape of neighboring genes by enhancer adoption

\section{Fares Taie ${ }^{\text {, B. Nedelec }}{ }^{1}$, P. David ${ }^{I}$, C. Angée ${ }^{1}$, S. Crippa ${ }^{2}$, B. Passet ${ }^{3}, J$. Vilotte $^{3}$, N. Chassaing ${ }^{4}$, J. Kaplan', C. Kostic ${ }^{5}$, P. Calvas ${ }^{4}$, J. Rozet ${ }^{I}$}

${ }^{1}$ INSTITUTE IMAGINE, Paris, France, ${ }^{2}$ University of Lausanne, Lausanne, Switzerland, ${ }^{3}$ UMR1313-INRA, Paris, France, ${ }^{4}$ Service de Génétique Clinique, Hôpital Purpan, Toulouse, France, ${ }^{5}$ University of Lausanne, Lausanne, France

Introduction: Previously, we reported 13q32.1 deletions as the cause of congenital microcoria (MCOR), a rare dominant disorder of iris development characterized by absent dilator muscle. GPR180 and TGDS were consistently altered, but the loss of either one individually does not cause MCOR. The genomic architecture of the 13q32.1 region is consistent with the existence of adjacent TADs, 
the boundary of which could be altered by MCOR deletions.

Methods: We generated mouse models carrying the minimal MCOR deletion (35KB) or three smaller deletions within this region encompassing binding sites for the insulator CTCF, respectively and analyzed their morphological, histological and molecular phenotypes.

Results: Heterozygous animals carrying the $35 \mathrm{~KB}$ deletion display moderate reduction in pupil diameter and ectopic expression of a neighboring gene in the iris starting during embryonic life, before the formation of the dilator muscle, through to adulthood. Smaller deletions encompassing putative insulator sites do not cause ectopic expression of the neighboring gene. This suggests that the $35 \mathrm{~KB}$ MCOR deletion may lead to the adoption of a normally distant enhancer by the deregulated gene, due to reduced distance between the two, instead of a TADboundary disruption. $4 \mathrm{C}$ sequencing to characterize the chromosomal interactions generated by the $35 \mathrm{~KB}$ deletion and the generation of mice lacking an excellent candidate enhancer are ongoing, to assess this hypothesis.

Conclusion: Here, we show that MCOR deletions alter the genomic architecture of $13 \mathrm{q} 32.1$ and gene expression in the developing iris, changing the fate of the progenitors that should give rise to the dilator muscle.

L. Fares Taie: None. B. Nedelec: None. P. David: None. C. Angée: None. S. Crippa: None. B. Passet: None. J. Vilotte: None. N. Chassaing: None. J. Kaplan: None. C. Kostic: None. P. Calvas: None. J. Rozet: None.

\section{C19.5}

First estimation of the scale of canonical $5^{\prime}$ splice site GT $>$ GC mutations generating wild-type transcripts and their medical genetic implications

J. H. Lin ${ }^{1,2,3}$, X. Y. Tang ${ }^{2,3}$, A. Boulling ${ }^{1}$, W. B. Zou ${ }^{2,3}$, E. Masson ${ }^{1,4}$, Y. Fichou ${ }^{1}$, L. Raud ${ }^{1}$, M. Le Tertre ${ }^{I}$,

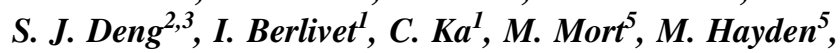
G. Le Gac ${ }^{1}$, D. N. Cooper ${ }^{5}$, Z. S. $\mathrm{Li}^{2}$, C. Férec ${ }^{1,4}$, Z. Liao $^{2,3}$, J. M. Chen ${ }^{I}$

${ }^{1}$ EFS, Univ Brest, Inserm, UMR 1078, GGB, Brest, France, ${ }^{2}$ Department of Gastroenterology, Changhai Hospital, the Second Military Medical University, Shanghai, China, ${ }^{3}$ Shanghai Institute of Pancreatic Diseases, Shanghai, China, ${ }^{4}$ CHU Brest, Service de Génétique, Brest, France, ${ }^{5}$ Institute of Medical Genetics, School of Medicine, Cardiff University, Cardiff, United Kingdom

It has long been known that canonical $5^{\prime}$ splice site (5'SS) GT $>$ GC mutations may be compatible with normal splicing. However, to date, the true scale of canonical 5'SS GT>GC mutations generating wild-type transcripts, both in the context of the frequency of such mutations and the level of wild-type transcripts generated from the mutation alleles, remain unknown. Herein, combining data derived from a meta-analysis of 45 informative disease-causing $5^{\prime} \mathrm{SS}$ GT>GC mutations (from 42 genes) and a cell culturebased full-length gene splicing assay of $1035^{\prime}$ SS GT>GC mutations (from 30 genes), we estimate that $\sim 15-18 \%$ of the canonical GT $5^{\prime}$ SSs are capable of generating between 1 and $84 \%$ normal transcripts as a consequence of the substitution of GT by GC. We further demonstrate that the canonical $5^{\prime} \mathrm{SSs}$ whose substitutions of GT by GC generated normal transcripts show stronger complementarity to the $5^{\prime}$ end of U1 snRNA than those sites whose substitutions of GT by GC did not lead to the generation of normal transcripts. We also observed a correlation between the generation of wild-type transcripts and a milder than expected clinical phenotype but found that none of the available splicing prediction tools were able to accurately predict the functional impact of 5'SS GT>GC mutations. Our findings imply that 5'SS GT>GC mutations may not invariably cause human disease but should also help to improve our understanding of the evolutionary processes that accompanied GT $>$ GC subtype switching of U2-type introns in mammals.

J.H. Lin: None. X.Y. Tang: None. A. Boulling: None. W.B. Zou: None. E. Masson: None. Y. Fichou: None. L. Raud: None. M. Le Tertre: None. S.J. Deng: None. I. Berlivet: None. C. Ka: None. M. Mort: None. M. Hayden: None. G. Le Gac: None. D.N. Cooper: None. Z.S. Li: None. C. Férec: None. Z. Liao: None. J.M. Chen: None.

\section{C19.6}

Novel regulatory elements control translation of key stress response factors linked to disease

\section{J. Rendleman ${ }^{I}$, V. Hronová ${ }^{2}$, D. Sultanov ${ }^{I}$, S. Maity ${ }^{1}$, A. Lei ${ }^{1}$, M. Hatzoglou ${ }^{3}$, L. Valášek ${ }^{2}, C$. Vogel ${ }^{1}$ \\ ${ }^{I}$ New York University, New York, NY, United States, ${ }^{2}$ Institute of Microbiology ASCR, Prague, Czech Republic, ${ }^{3}$ Case Western Reserve University, Cleveland, $\mathrm{OH}$, United States}

Introduction: The accumulation of misfolded proteins in the endoplasmic reticulum, known as ER stress, is a hallmark of many diseases including cancer. To combat ER stress, cells employ the Unfolded Protein Response, which includes translation induction of stress response factors. For a subset this induction is achieved through upstream open reading frames in the $5^{\prime}$ UTR, however mechanisms are not well understood for many others. 
Material and Methods: We performed ribosome profiling in human cells undergoing ER stress and searched $5^{\prime}$ UTRs for ribosome-enriched motifs. Using reporters, we tested significant motifs for their impact on protein synthesis under stress. To further characterize their function, we used the CRISPR/Cas9 system to engineer mutations in cell lines.

Results: We identified a short motif in the $5^{\prime}$ UTR with significant enrichment in ribosomal occupancy; reporter assays confirmed the functionality of this sequence. This new element is common among transcriptional regulators, including a key factor of the Unfolded Protein Response, and correlates with reduced translation of downstream coding regions, suggesting a translation inhibitory element. Importantly, cross-referencing with The Cancer Genome Atlas revealed overlap with cancer risk variants.

Conclusion: The $5^{\prime}$ UTR is a critical component of a gene; however, much is unknown regarding its role in expression, particularly through translation. Traditional cancer exome studies miss mutations in undescribed regulatory regions in the UTR. The element we have discovered not only has the potential to revise a long-standing model in the field of translation regulation, but also highlights novel mutations that may contribute to oncogenesis.

J. Rendleman: None. V. Hronová: None. D. Sultanov: None. S. Maity: None. A. Lei: None. M. Hatzoglou: None. L. Valášek: None. C. Vogel: None.

\section{C20 Neuromuscular and neurodegenerative disorders}

\section{C20.1}

Large clinical cohort undergoing simultaneous single nucleotide and copy number variant analysis reveals broad mutation spectrum and high diagnostic yield for neuromuscular disorders

\section{E. Decker ${ }^{1}$, T. L. Winder ${ }^{1}$, C. A. Tan ${ }^{1}$, S. Klemm ${ }^{1}$, H. White ${ }^{I}$, J. M. Westbrook ${ }^{I}, J$. Wang ${ }^{1}$, A. Entezam ${ }^{I}$, R. Truty ${ }^{1}$, E. McNally ${ }^{2}$, S. Aradhya ${ }^{1}$}

${ }^{1}$ Invitae, San Francisco, CA, United States, ${ }^{2}$ Center for Genomic Medicine, Northwestern University, Chicago, IL, United States

Molecular genetic testing for hereditary neuromuscular disorders has been increasingly used to confirm clinical diagnoses, identify subtype, and inform management and prognosis. These important uses of genetic testing have been corroborated in several small studies, but large data sets are needed to fully illuminate the utility and complexity of genetic analysis. Moreover, universal high-resolution analysis of intragenic copy number variation $(\mathrm{CNV})$ is now possible, enabling us to evaluate its contribution to neuromuscular disorders.

We investigated a cohort of 10,782 unrelated individuals with a range of neuromuscular disorders using highcoverage next-generation sequencing (NGS) with simultaneous sequence and exon-level CNV detection for subsets of 232 genes related to these disorders to better understand diagnostic yield, mutation spectrum, and complexities of variant interpretation.

Definitive diagnosis was obtained in 2,484 individuals (23\%). Diagnostic yields ranged from $10 \%$ for dystonia to $53 \%$ for spinal muscular atrophy or dystrophinopathy. As expected, $94.8 \%$ of pathogenic CNVs were found in SMN1, PMP22, and DMD, however, the remaining pathogenic CNVs were found across 31 other genes and intragenic CNVs accounted for as much as $20 \%$ of all clinically significant results. Separately, we evaluated the rate of reclassification of variants of uncertain significance and observed that up to $15 \%$ of such variants in some genes were reclassified to likely pathogenic or pathogenic.

Our data represent one of the largest studies demonstrating the utility of NGS-based genetic testing in neuromuscular disorders and the important contribution of exonlevel $\mathrm{CNV}$ analysis to inform clinical management of individuals with these disorders.

E. Decker: A. Employment (full or part-time); Significant; Invitae. T.L. Winder: A. Employment (full or part-time); Significant; Invitae. C.A. Tan: A. Employment (full or part-time); Significant; Invitae. S. Klemm: A. Employment (full or part-time); Significant; Invitae. H. White: A. Employment (full or part-time); Significant; Invitae. J.M. Westbrook: A. Employment (full or parttime); Significant; Invitae. J. Wang: A. Employment (full or part-time); Significant; Invitae. A. Entezam: A. Employment (full or part-time); Significant; Invitae. R. Truty: A. Employment (full or part-time); Significant; Invitae. E. McNally: F. Consultant/Advisory Board; Significant; Invitae. S. Aradhya: A. Employment (full or parttime); Significant; Invitae.

\section{C20.2}

Mutations in the Golgi protein GBF1 as a novel cause of distal hereditary motor neuropathy

N. Mendoza Ferreira ${ }^{1,2,3}$, M. Karakaya ${ }^{1,2,3}$, I. Hölker ${ }^{1,2,3}$, D. Beijer ${ }^{4,5}$, B. Schrank ${ }^{6}$, K. Brigatti ${ }^{7}$, C. GonzagaJauregui $^{8}$, E. Puffenberger ${ }^{7}$, G. Wunderlich ${ }^{9}$, P. De Jonghe $^{4,5,10}$, T. Deconinck ${ }^{4,5}$, K. Strauss ${ }^{7}$, J. Baets $^{4,5,10}$, B. Wirth ${ }^{1,2,3}$

${ }^{1}$ Institute of Human Genetics, University of Cologne, Cologne, Germany, ${ }^{2}$ Center for Molecular Medicine Cologne, Cologne, Germany, ${ }^{3}$ Center for Rare Diseases 
Cologne, Cologne, Germany, ${ }^{4}$ Neurogenetics Group, Center for Molecular Neurology, VIB, Antwerp, Belgium, ${ }^{5}$ Laboratory of Neuromuscular Pathology, Institute BornBunge, University of Antwerp, Antwerp, Belgium, ${ }^{6} \mathrm{DKD}$ HELIOS Kliniken, Department of Neurology, Wiesbaden, Germany, ${ }^{7}$ Clinic for Special Children, Strasburg, PA, United States, ${ }^{8}$ Regeneron Genetics Center, Regeneron Pharmaceuticals Inc, Tarrytown, NY, United States, ${ }^{9}$ Department of Neurology, University Hospital Cologne, Cologne, Germany, ${ }^{10}$ Neuromuscular Reference Centre, Department of Neurology, Antwerp University Hospital, Antwerp, Belgium

Introduction: Distal hereditary motor neuropathies (dHMN) are clinically and genetically heterogeneous diseases characterized by motor neuron (MN) degeneration and progressive muscle atrophy. For $50 \%$ of individuals with dHMN, the disease-causing gene is unknown.

Materials and Methods: WES and WGS were used to identify the monogenic causes of dHMN. Patient-derived fibroblasts were used to assess the functional consequence of candidate variants on splicing, protein abundance, and subcellular localization.

Results: We identified putative pathogenic variants in GBF1 (Golgi Brefeldin-A-Resistant Guanine Nucleotide Exchange Factor-1) in four unrelated families with dominant or sporadic dHMN. Other known dHMN-implicated genes were excluded. De novo or inherited mutations (c.2945G>A, p.Cys982Tyr; c.3410C>T, p.Ala1137Val; c. $4382 \mathrm{G}>$ A, p.Arg1461Gln; and c.3524G $>$ A, p.Trp1175*) were associated with slowly progressive distal muscle weakness and atrophy accompanied by musculoskeletal deformities (scoliosis, pes cavus, hammer toes). Electrophysiological studies consistently revealed axonal motor neuropathy; three patients also had distal sensory loss. GBF1 plays a pivotal role in COPI-vesicular trafficking and Golgi maintenance. Functional studies confirmed that the variants c.3410C $>\mathrm{T}$, located after the $3^{\prime}$ splice acceptor site (SAS), and c.4382G $>$ A, predicted to introduce a novel SAS, do not affect splicing. Protein levels from patients fibroblasts confirmed GBF1 haploinsufficiency for the W1175* mutation, whereas A1137V and R1461Q exhibited moderately increased GBF1 levels. Preliminary immunostaining analyses revealed Golgi fragmentation in fibroblast lines p.Ala1137Val and p.Arg1461Gln.

Conclusion: Previous studies have linked disrupted COPI-transport and Golgi fragmentation with other motor neuropathies. Our results emphasize the role of GBF1 in these contexts and indicate that GBF1 mutations are a novel cause of dHMN.

N. Mendoza Ferreira: None. M. Karakaya: None. I. Hölker: None. D. Beijer: None. B. Schrank: None. K. Brigatti: None. C. Gonzaga-Jauregui: None. E.
Puffenberger: None. G. Wunderlich: None. P. De Jonghe: None. T. Deconinck: None. K. Strauss: None. J. Baets: None. B. Wirth: None.

\section{C20.3}

Recessive mutations in muscle-specific isoforms of $F X R 1$ cause congenital multi-minicore myopathy

E. Fernández-Núñez ${ }^{1}$, M. Estañ ${ }^{1,2}$, M. S. Zaki ${ }^{3}$, M. Esteban ${ }^{4}$, S. Donkervoort ${ }^{5}$, C. Hawkins ${ }^{6}$, J. A. Caparros-Martin ${ }^{1,2,7}$, D. Saade ${ }^{5}, \mathrm{Y}_{\text {. }} \mathrm{Hu}^{5}, \mathrm{~V}$. Bolduc ${ }^{5}$, K. Chao ${ }^{8}$, J. Nevado ${ }^{9}$, A. Lamuedra ${ }^{10}$, R. Largo ${ }^{10}$, G. Herrero-Beaumont ${ }^{10}$, J. Regadera ${ }^{11}$, C. HernándezChico $^{2,12}$, E. F. Tizzano ${ }^{2,13}$, V. Martinez-Glez, ${ }^{2,9}$, J. J. Carvajal $^{14}$, R. Zong ${ }^{15}$, D. Nelson ${ }^{15}$, G. A. Otaify ${ }^{3}$, S. Temtamy ${ }^{3}$, M. Aglan ${ }^{3}$, M. Issa ${ }^{3}, C$. G. Bönnemann ${ }^{5}$, P. Lapunzina ${ }^{2,9}$, G. Yoon ${ }^{16,17}$, V. L. Ruiz-Perez ${ }^{1,2}$

${ }^{1}$ Instituto de Investigaciones Biomédicas "Alberto Sols" (CSIC-UAM), Madrid, Spain, ${ }^{2}$ CIBER de enfermedades raras (CIBERER-ISCIII), Madrid, Spain, ${ }^{3}$ Department of Clinical Genetics. Human Genetics and Genome Research Division, Centre of Excellence of Human Genetics, National Research Centre, Cairo, Egypt, ${ }^{4}$ Departamento de Anatomía Patológica. Hospital Universitario La PazIdiPaz-UAM, Madrid, Spain, ${ }^{5}$ Neuromuscular and Neurogenetic Disorders of Childhood Section, National Institute of Neurological Disorders and Stroke, National Institutes of Health, Bethesda, MD, United States, ${ }^{6}$ Division of Pathology, Department of Paediatric Laboratory Medicine, The Hospital for Sick Children, University of Toronto, Toronto, ON, Canada, ${ }^{7}$ Scholl of Pharmacy and Biomedical Sciences and Curtin Health Innovation Research Institute, Curtin University, Perth, Australia, ${ }^{8}$ Center for Mendelian Genomics, Program in Medical and Population Genetics, Broad Institute of MIT and Harvard, Boston, MA, United States, ${ }^{9}$ Instituto de Genética Médica y Molecular (INGEMM), Hospital Universitario La Paz-IdiPaz-UAM, Madrid, Spain, ${ }^{10}$ Bone and Joint Research Unit, The Institution of Health Research (IIS)-Fundación Jiménez Díaz, UAM, Madrid, Spain, ${ }^{11}$ Departamento de Anatomía, Histología y Neurociencia, Facultad de Medicina, Universidad Autónoma de Madrid, Madrid, Spain, ${ }^{12}$ Unidad de Genética Molecular, Hospital Ramón y Cajal, Madrid, Spain, ${ }^{13}$ Department of Clinical and Molecular Genetics and Rare Diseases Unit, Hospital Vall d'Hebron, Barcelona, Spain, ${ }^{14}$ Centro Andaluz de Biología del Desarrollo (CSIC-UPO-JA), Universidad Pablo de Olavide, Sevilla, Spain, ${ }^{15}$ Department of Molecular and Human Genetics, Jan and Dan Duncan Neurological Research Institute, Baylor College of Medicine, Moursund Street, Houston, TX, United States,

${ }^{16}$ Division of Clinical and Metabolic Genetics, Department of Paediatrics, The Hospital for Sick Children, University of 
Toronto, Toronto, ON, Canada, ${ }^{17}$ Division of Neurology, Department of Paediatrics, The Hospital for Sick Children, University of Toronto, Toronto, ON, Canada

FXRI is an alternatively spliced gene encoding several protein isoforms (FXR1P) with RNA-binding properties. Unlike other tissues, cardiac and skeletal muscle only express two FXR1P isoforms that incorporate an additional exon-15. Using exome sequencing, we identified recessive mutations in the muscle-specific exon-15 of FXR1 in two unrelated families with congenital myopathy, one of them having a neonatal lethal phenotype. To investigate the pathogenicity of exon-15 mutations, we generated a CRISPR-Cas9 mouse model carrying the same mutation as the family with lethal phenotype (delACAG) and a different exon-15 mutant (dupA) that results in low FXR1P levels. Mutant homozygous mice were found with decreased muscle volume, muscle strength and bone density by MRI, behavioural tests and DEXA respectively, while histopathological studies showed muscle fibers of reduced diameter, increased centralized nuclei, and type I predominance. Ultra-structural analysis of fibers from mutant mice also revealed multiple cores and Z-band disruption. Notably, phenotypic severity was greater in delACAG mutants than in dupA mice. Histopathology of a triceps biopsy from a FXRI patient was consistent with these findings. We additionally demonstrate that the delACAG mutation leads to abnormal FXR1P isoforms which accumulate in a new type of cytoplasmic granules in human and mouse muscle cells. In conclusion, we report that mutations in exon-15 of FXRl are associated with a multi-minicore myopathy which varies in severity depending on to the effect of each mutation on the protein. This is the first time that mutations in FXRI are demonstrated to be associated with a human condition. Funding: MINECO/ CIBERER/NINDS

E. Fernández-Núñez: None. M. Estañ: None. M.S. Zaki: None. M. Esteban: None. S. Donkervoort: None. C. Hawkins: None. J.A. Caparros-Martin: None. D. Saade: None. Y. Hu: None. V. Bolduc: None. K. Chao: None. J. Nevado: None. A. Lamuedra: None. R. Largo: None. G. Herrero-Beaumont: None. J. Regadera: None. C. Hernández-Chico: None. E.F. Tizzano: None. V. Martinez-Glez: None. J.J. Carvajal: None. R. Zong: None. D. Nelson: None. G.A. Otaify: None. S. Temtamy: None. M. Aglan: None. M. Issa: None. C.G. Bönnemann: None. P. Lapunzina: None. G. Yoon: None. V.L. RuizPerez: None.

\section{C20.4}

Novel mutations in MYBPC1 associated with myogenic tremor
J. Stavusis ${ }^{1}$, J. Geist ${ }^{2}$, B. Lace ${ }^{1,3}$, N. Wright ${ }^{4}$, D. Haubenberger ${ }^{5}$, C. Bonneman ${ }^{6}, C$. Ward $^{7}$, A. Kontrogianni-Konstantopoulos ${ }^{2}$

${ }^{1}$ Latvian Biomedical Study and Research Centre, Riga, Latvia, ${ }^{2}$ Univeristy of Maryland School of Medicine, Department of Biochemistry and Molecular Biology, Baltimore, MD, United States, ${ }^{3}$ Centre Hospitalier Universitaire de Quebec, Quebec city, QC, Canada, ${ }^{4} J a m e s$ Madison University, Department of Chemistry and Biochemistry, Harrisonburg, VA, United States, ${ }^{5}$ Clinical Trials Unit, Office of the Clinical Director, NINDS Intramural Research Program, National Institute of Neurological Disorders and Stroke, NIH, Bethesda, MD, United States, ${ }^{6}$ Neuromuscular and Neurogenetic Disorders of Childhood Section, Neurogenetics Branch, National Institute of Neurological Disorders and Stroke, NIH, Bethesda, MD, United States, ${ }^{7}$ University of Maryland Baltimore, Department of Orthopedics, Baltimore, MD, United States

Introduction: $M Y B P C 1$ encodes slow Myosin Binding Protein-C (sMyBP-C), a modular sarcomeric protein with structural and regulatory roles expressed in skeletal muscles. sMyBP-C interacts with both actin and myosin filaments in a dynamic manner. Recently our studies identified novel mutations in $M Y B P C l$ associated with a new form of myopathy.

Materials and Methods: Exome sequencing and in silico prognostic tools were used to identify novel $M Y B P C 1$ pathogenic variants. Biochemical assays and molecular dynamics were performed to examine the impact of the $M Y B P C 1$ variants on actomyosin binding, the structure and stability of sMyBP-C. A knock-in mouse model containing one of the pathogenic mutations was generated.

Results: We have identified novel autosomal dominant mutations in $M Y B P C 1$ that co-segregate with a new form of myopathy characterized by muscle weakness, hypotonia, facial/body deformities, and tremor of possibly myogenic origin. The individual mutations differentially affect the stability, structure and ability of sMyBP-C to bind myosin. The generated knock-in mouse model faithfully recapitulates the myopathic phenotype and disease progression observed in patients. Heterozygous knock-in mice are significantly smaller and exhibit behavioural, morphological and contractile deficits compared to their wild-type littermates.

Conclusion: Our work expands the range of myopathies associated with $M Y B P C 1$ mutations, which was limited to severe and lethal forms of distal arthrogryposis. We now present evidence for a $M Y B P C 1$-linked myopathy associated with tremor. 
This work was supported by NIH/NIAMS (T32AR00759217 to JG \& R21AR072981 to AKK), Muscular Dystrophy Association (313579 to AKK), European Regional Development Fund (No. 2010/0223/2DP/2.1.1.1.0/10/APIA/VIAA/ 025 to BL) and FULBRIGHT PROGRAM (PS00266997 to JS).

J. Stavusis: None. J. Geist: None. B. Lace: None. N. Wright: None. D. Haubenberger: None. C. Bonneman: None. C. Ward: None. A. KontrogianniKonstantopoulos: None.

\section{C20.5}

Absence of NFASC isoform NF186 causes an autosomal recessive ataxia syndrome

M. Kvarnung ${ }^{1,2}$, M. Shahsavani ${ }^{3}$, F. Taylan ${ }^{1}$, N. Breeuwsma ${ }^{3}$, L. Laan ${ }^{4}$, J. Schuster ${ }^{4}$, Z. Jin ${ }^{5}$, D. Nilsson ${ }^{1,2}$, A. Lieden ${ }^{1,2}$, B. Anderlid ${ }^{1,2}$, M. Nordenskjöld ${ }^{1,2}$, E. Syk Lundberg ${ }^{1,2}$, B. Birnir ${ }^{5}$, N. Dahl ${ }^{4}$ A. Nordgren ${ }^{1,2}$, A. Lindstrand ${ }^{1,2}$, A. Falk $^{3}$

${ }^{1}$ Dept of Molecular Medicine and Surgery, Karolinska Institutet, Stockholm, Sweden, ${ }^{2}$ Dept of Clinical Genetics, Karolinska University Hospital, Stockholm, Sweden, ${ }^{3}$ Dept of Neuroscience, Karolinska Institutet, Biomedicum, Stockholm, Sweden, ${ }^{4}$ Dept of Immunology, Genetics and Pathology, Science for Life Laboratory, Uppsala University, Biomedical Centre, Uppsala, Sweden, ${ }^{5}$ Dept of Neuroscience, Biomedical Centre, Uppsala University, Uppsala, Sweden

The etiology of hereditary ataxia syndromes is heterogeneous and the mechanisms underlying these disorders are often unknown. Here, we utilized exome sequencing in two siblings with progressive ataxia and muscular weakness and identified a novel homozygous mutation (c.3020-1G>A) in neurofascin (NFASC). In RNA extracted from fibroblasts we showed that the mutation resulted in inframe skipping of exon 26, with deprived expression of the transcript that corresponds to NFASC isoform NF186. To further investigate the disease mechanisms, we assessed the NFASC mutation in our established in vitro neural cell model. Fibroblasts from one affected sibling and the unaffected parents were reprogrammed to pluripotent stem cells (iPSCs), directed to long-term neuroepithelial stem cells (NESCs) and finally differentiated to neurons. In early neurogenesis, differentiating cells with a selective depletion of the NF186 isoform showed significantly reduced neurite outgrowth as well as fewer emerging neurites. Furthermore, whole-cell patch-clamp recordings of patient derived neural cells revealed a lower threshold for openings, indicating altered $\mathrm{Na}^{+}$channel kinetics suggesting a lower threshold for openings as compared to neural cells without the
NFASC mutation. Taken together, our results strongly suggest that loss of NFASC isoform NF186 causes perturbed neurogenesis and impaired neuronal biophysical properties resulting in a novel early onset autosomal recessive ataxia syndrome.

Funding: Financial support was provided by grants from the Swedish Medical Research Council and the Stockholm County Council. Grant from Swedish Research Council (2015-02424, 2017-03407, 2017-02936), Stiftelsen för strategisk forskning, SSF (IB13-0074), Hjärnfonden, The Hållsten Research Foundation and Stiftelsen Sävstaholm.

M. Kvarnung: None. M. Shahsavani: None. F. Taylan: None. N. Breeuwsma: None. L. Laan: None. J. Schuster: None. Z. Jin: None. D. Nilsson: None. A. Lieden: None. B. Anderlid: None. M. Nordenskjöld: None. E. Syk Lundberg: None. B. Birnir: None. N. Dahl: None. A. Nordgren: None. A. Lindstrand: None. A. Falk: None.

C20.6

Peripheral monitoring of neurodegeneration using cellfree DNA methylation

Z. Chatterton ${ }^{1,2}$, R. Landin-Romero ${ }^{1}$, C. T. Liang ${ }^{1}$, K. Phan', W. Carr ${ }^{3}$, G. Kamimori ${ }^{3}$, M. Beeri ${ }^{2}$, Y. Ge ${ }^{2}$, A. Dwork ${ }^{4}$, F. Haghighi ${ }^{2,5}$, O. Piguet ${ }^{1}$, G. Halliday ${ }^{1}$, J. Kwok ${ }^{1}$

${ }^{I}$ The University of Sydney, Camperdown, Australia, ${ }^{2}$ Icahn School of Medicine at Mount Sinai, New York, NY, United States, ${ }^{3}$ Walter Reed Army Institute of Research, Silver Spring, MD, United States, ${ }^{4}$ Columbia University, New York, NY, United States, ${ }^{5}$ James J. Peters VA Medical Center, Bronx, NY, United States

Introduction: Neurodegeneration occurs in a variety of human diseases, but molecular profiling of the brain is difficult. Cell free DNA (cfDNA) derived from neurological tissue holds great potential for very early detection and monitoring of neurodegeneration.

Materials and Methods: We characterised DNA methylation specific to human brain-cells and brain-region using genome-wide epigenetic profiles and compared these with targeted deep-sequencing (bisulfite) of cfDNA methylation in acute and chronic neurodegenerative disease subjects. To detect brain-derived cfDNA we developed a new statistical approach for analysing DNA methylation at the single-molecule level using k-mers.

Results: We detected an acute appearance in cfDNA from dorsolateral prefrontal cortex neurons (DLPFC-neuron) following blast exposure within a unique cohort of active duty military personnel involved in operational breaching. We also detected brain-derived cfDNA during cognitive decline within a unique cohort of type- 2 diabetic patients 
with and without cognitive decline in which we identified stage-dependent patterns of DLPFC-neuron cfDNA and the presence of cerebellar-derived cfDNA. Finally, we identified significant $(\mathrm{p}<0.05)$ DLPFC-neuron cfDNA among patients with DLPFC neurodegeneration validated by longitudinal structural magnetic resonance brain-imaging.

Conclusion: Here we report the first evidence of cerebral cortical neuron-derived cfDNA and cerebellar cfDNA within acute neurotrauma and chronic neurodegeneration, establishing the first class of peripheral biomarkers with specificity for the cell-type and brain-region undergoing injury or neurodegeneration.

Funding: VA Merits I01RX001705 and I01CX001395. NIDA T32 postdoctoral training fellowship (3 T32 DA 7135-31-S1). Sample collections were supported by Broad Agency Announcement Award No. W81XWH-16-2-0001.

Z. Chatterton: None. R. Landin-Romero: None. C.T. Liang: None. K. Phan: None. W. Carr: None. G. Kamimori: None. M. Beeri: None. Y. Ge: None. A. Dwork: None. F. Haghighi: None. O. Piguet: None. G. Halliday: None. J. Kwok: None.

\section{C21 Internal organs}

\section{C21.1}

Loss-of-function variants in myocardin cause congenital megabladder in humans and mice

A. Houweling ${ }^{1}$, G. Beaman ${ }^{2}$, A. Postma ${ }^{3}$, B. Gainous ${ }^{4}$, K. Lichtenbelt ${ }^{5}$, F. Brancati ${ }^{6}$, F. Lopes ${ }^{7}$, I. van der Made ${ }^{8}$, A. Polstra ${ }^{9}$, M. Robinson ${ }^{10}$, K. Wright ${ }^{11}$, A. Jackson ${ }^{12}$, R. Genesio ${ }^{13}$, L. Camerota' ${ }^{14}$, E. D’Angelo ${ }^{15}$, E. MeijersHeijboer $^{16}$, V. Christoffels ${ }^{17}$, K. McHugh $^{18}$, B. Black ${ }^{19}$, W. Newman ${ }^{20}$, A. Woolf $^{21}$, E. Creemers ${ }^{8}$

${ }^{1}$ Department of Clinical Genetics, Amsterdam UMC, Vrije Universiteit Amsterdam, Amsterdam, Netherlands, ${ }^{2}$ Division of of Evolution and Genomic Sciences, School of Biological Sciences, Faculty of Biology Medicine and Health, University of Manchester, Manchester, United Kingdom, ${ }^{3}$ Department of Medical Biology, Amsterdam Cardiovascular Sciences, Amsterdam UMC, Academic Medical Center, Department of Clinical Genetics, Amsterdam Cardiovascular Sciences, Amsterdam UMC, Academic Medical Center, Amsterdam, Netherlands, ${ }^{4}$ Cardiovascular Research Institute, University of California, San Francisco, San Francisco, CA, USA, San Francisco, CA, United States, ${ }^{5}$ Department of Medical Genetics, University Medical Center Utrecht, Utrecht, Netherlands, ${ }^{6}$ Laboratory of Molecular and Cell Biology, Istituto Dermopatico dell'Immacolata, IDI-IRCCS, Department of Life, Health and Environmental Sciences, University of L'Aquila, Rome,
Italy, ${ }^{7}$ Division of Cell Matrix Biology and Regenerative Medicine, School of Biological Sciences, Faculty of Biology Medicine and Health, University of Manchester, Manchester, United Kingdom, ${ }^{8}$ Department of Experimental Cardiology, Amsterdam Cardiovascular Sciences, Amsterdam UMC, Academic Medical Center, Amsterdam, Netherlands, ${ }^{9}$ Department of Clinical Genetics, Amsterdam UMC, Vrije Universiteit Amsterdam, Laboratory Genetic Metabolic Diseases, Departments of Clinical Chemistry and Clinical Genetics, Academic Medical Center, University of Amsterdam, Amsterdam, Netherlands, ${ }^{10}$ Department of Biology, Miami University, Oxford, Columbus, OH, United States, ${ }^{11}$ Department of Biology, Miami University, Oxford, Oxford, OH, United States, ${ }^{12}$ Center for Clinical and Translational Research, The Research Institute, Nationwide Children's Hospital, Columbus, Columbus, OH, United States, ${ }^{13}$ Department of Molecular Medicine and Medical Biotechnology, University of Naples Federico II, Naples, Italy, ${ }^{14}$ Medical Genetics Division, Department of Life, Health and Environmental Sciences, University of L'Aquila, L'Aquila, Italy, ${ }^{15}$ Pathology Division, Department of Life, L'Aquila, Italy, ${ }^{16}$ Department of Clinical Genetics, Amsterdam UMC, Vrije Universiteit Amsterdam, Amsterdam, The Netherlands, Amsterdam, Netherlands, ${ }^{17}$ Department of Medical Biology, Amsterdam Cardiovascular Sciences, Amsterdam UMC, Academic Medical Center, Amsterdam, Netherlands, ${ }^{18}$ Center for Clinical and Translational Research, The Research Institute, Nationwide Children's Hospital, Columbus, OH, United States, ${ }^{19}$ Cardiovascular Research Institute, University of California, San Francisco, $C A$, United States, ${ }^{20}$ Division of Evolution and Genomic Sciences, School of Biological Sciences, Faculty of Biology Medicine and Health, University of Manchester, Manchester Centre for Genomic Medicine, Manchester University NHS Foundation Trust, Manchester Academic Health, Manchester, United Kingdom, ${ }^{21}$ Division of Cell Matrix Biology and Regenerative Medicine, School of Biological Sciences, Faculty of Biology Medicine and Health, University of Manchester, Royal Manchester Children's Hospital, Manchester University NHS Foundation Trust, Manchester Aca, Manchester, United Kingdom

Myocardin (MYOCD) is the founding member of a class of transcriptional co-activators that bind serum response factor to activate gene expression programs coding for contractile and other cytoskeletal proteins critical in smooth- and cardiac muscle development. Insights into the molecular functions of MYOCD have been obtained from cell culture studies and, to date, our in vivo knowledge about MYOCD comes exclusively from experimental animals. For example, expression of dominant negative Myocd disrupts myocardial differentiation in embryonic frogs, while Myocd null mice die midgestation with failed vascular smooth muscle (SM) 
cell differentiation. Here, we define a devastating and often lethal human congenital disease associated with inheritance of pathogenic MYOCD variants, with 14 affected individuals in four families. This disease manifests as a massively dilated urinary bladder, or megabladder, with a disrupted SM wall. These human results are supported by evidence of megabladder in two distinct mouse models with reduced Myocd levels. Together, these findings highlight a vital role for MYOCD in mammalian organogenesis. In conclusion, we demonstrate for the first time that variations in MYOCD result in human disease, and propose that loss-of-function variants in MYOCD cause semi-dominant congenital megabladder. This has important implications for genetic counseling of families with congenital megabladder, sheds new light on bladder development, and expands the pathophysiological spectrum of inherited SM disorders.

A. Houweling: None. G. Beaman: None. A. Postma: None. B. Gainous: None. K. Lichtenbelt: None. F. Brancati: None. F. Lopes: None. I. van der Made: None. A. Polstra: None. M. Robinson: None. K. Wright: None. A. Jackson: None. R. Genesio: None. L. Camerota: None. E. D'Angelo: None. E. Meijers-Heijboer: None. V. Christoffels: None. K. McHugh: None. B. Black: None. W. Newman: None. A. Woolf: None. E. Creemers: None.

\section{C21.2}

Rare heterozygous deleterious GDF6 variants in patients with renal anomalies

H. Martens ${ }^{1}$, I. Hennies ${ }^{2}$, M. Getwan ${ }^{3}$, A. Kosfeld ${ }^{1}$,

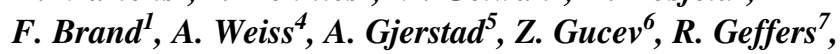

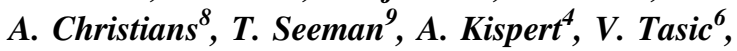
A. Bjerre ${ }^{5}$, S. S. Lienkamp ${ }^{3}$, D. Haffner ${ }^{2}$, R. G. Weber ${ }^{1}$

${ }^{1}$ Department of Human Genetics, Hannover Medical School, Hannover, Germany, ${ }^{2}$ Department of Pediatric Kidney, Liver and Metabolic Diseases, Hannover Medical School, Hannover, Germany, ${ }^{3}$ Department of Medicine, Renal Division, Medical Center, University of Freiburg, Freiburg, Germany, ${ }^{4}$ Institute of Molecular Biology, Hannover Medical School, Hannover, Germany, ${ }^{5}$ Division of Paediatric and Adolescent Medicine, Oslo University Hospital, Oslo, Norway, ${ }^{6}$ Medical Faculty Skopje, University Children's Hospital, Skopje, Macedonia, The Former Yugoslav Republic of, ${ }^{7}$ Genome Analytics Research Group, Helmholtz Centre for Infection Research, Braunschweig, Germany, ${ }^{8}$ Institute of Pathology, Division of Neuropathology, Hannover Medical School, Hannover, Germany, ${ }^{9}$ Department of Paediatrics, Second Faculty of Medicine, Charles University, Prague, Czech Republic

Although around 50 genes are known to cause renal malformation when mutated, the genetic basis of most patients remains unsolved. In search of novel causative genes, we studied a family with renal in addition to skeletal, eye, ear malformations in one sibling, and ear anomalies in another sibling and their mother. Whole-exome sequencing yielded a rare heterozygous missense variant in the growth differentiation factor 6 (GDF6) gene known to cause autosomal-dominant Klippel-Feil syndrome type 1 (KFS1) in all affected family members. While skeletal and hearing abnormalities are characteristic of KFS1, renal anomalies have not been described as a hallmark. To assess whether GDF6 is involved in the pathogenesis of renal malformation, we performed mutational analysis of 193 further patients identifying two more cases with rare GDF6 missense variants. A CRISPR/Cas9-derived knockout in murine IMCD3 cells showing that Gdf6-deficiency attenuated cell migration was rescued by expression of human wildtype but not mutant GDF6, indicating variant pathogenicity with respect to a developmental phenotype. RNA in situ hybridization on murine embryo sections revealed expression of $G d f 6$ in the developing urogenital system. As gdf6 expression was also observed in the pronephros of Xenopus laevis, this model was used for in vivo gdf6 knockdown studies, resulting in impaired pronephros development in embryos with downregulated gdf6 expression. Altogether, we demonstrate rare heterozygous deleterious GDF6 variants in patients with renal malformation $(1.6 \%)$, and renal combined with ear, eye or skeletal anomalies (5.2\%), and provide evidence for an involvement of GDF6 in early nephrogenesis. (EKFS grant no. 2014_A234).

H. Martens: None. I. Hennies: None. M. Getwan: None. A. Kosfeld: None. F. Brand: None. A. Weiss: None. A. Gjerstad: None. Z. Gucev: None. R. Geffers: None. A. Christians: None. T. Seeman: None. A. Kispert: None. V. Tasic: None. A. Bjerre: None. S.S. Lienkamp: None. D. Haffner: None. R.G. Weber: None.

\section{C21.3}

Exome sequencing identifies phenocopies in every fifth solved case in a cohort of 174 patients with hereditary nephropathies

K. M. Riedhammer ${ }^{1,2}$, M. C. Braunisch ${ }^{2}$, R. Guenthner ${ }^{2}$, M. Wagner ${ }^{1,3,4}$, B. Uetz $^{5,6}$, B. Lange-Sperandio ${ }^{7}$, J. Hoefele ${ }^{I}$

${ }^{1}$ Institute of Human Genetics, Klinikum rechts der Isar, Technical University of Munich, Munich, Germany, ${ }^{2}$ Department of Nephrology, Klinikum rechts der Isar, Technical University of Munich, Munich, Germany, ${ }^{3}$ Institute of Neurogenomics, Helmholtz Zentrum München, Neuherberg, Germany, ${ }^{4}$ Institute of Human Genetics, Helmholtz Zentrum München, Neuherberg, Germany, 
Table 1 Overview of the study cohort

\begin{tabular}{lllllllll}
\hline & AS & ADTKD & CAKUT & Ciliopathy & FSGS/SRNS & VACTERL & Other & Total \\
\hline $\begin{array}{l}\text { Number of index cases (\% of cohort) } \\
\text { male (\%) }\end{array}$ & $34(20)$ & $6(3)$ & $30(17)$ & $19(11)$ & $49(28)$ & $9(5)$ & $27(16)$ & $174(100)$ \\
Median age and & 59 & 50 & 63 & 37 & 51 & 56 & 52 & 53 \\
$\begin{array}{l}\text { IQR at genetic testing in years } \\
\text { Diagnostic yield (\%) }\end{array}$ & $15(8.0-26.0)$ & $53(47.0-65.0)$ & $6(0.1-25.0)$ & $9(0.8-19.0)$ & $31(19.0-42.0)$ & $10(1.0-12.5)$ & $24(7.0-37.0)$ & $19(7.0-35.0)$ \\
Number of phenocopies (\% of disease group) & $3(9)$ & - & 27 & 47 & 16 & 0 & 30 & 30 \\
\hline
\end{tabular}

${ }^{5}$ München-Klinik Schwabing, Klinikum rechts der Isar, Technical University of Munich, Children's Hospital, Pediatric Nephrology, Munich, Germany, ${ }^{6} \mathrm{KfH}$ Center of Pediatric Nephrology, Munich, Germany, ${ }^{7}$ Department of Pediatric Nephrology, Dr. v. Hauner Children's Hospital, Ludwig-Maximilians University, Munich, Germany

Introduction: Hereditary nephropathies are both clinically and genetically heterogeneous disorders. In some patients the phenotype resembles a specific disease, but they do not carry the established genotype (phenocopy). Exome sequencing has been successfully employed in various hereditary kidney disorders. This study shows the spectrum and frequency of phenocopies identified by exome sequencing in a cohort encompassing monogenic inherited kidney disorders.

Materials and Methods: 174 index patients with a presumed hereditary kidney disease were recruited for exome sequencing and categorized in 7 disease groups according to their clinical presentation (Table 1). Variants were rated as per ACMG criteria. "Likely pathogenic" and "pathogenic" variants led to the designation "solved case".

Results: In 52/174 (30\%), a genetic diagnosis could be made, in 10/52 (19\%), a phenocopy could be identified (6\% of total cohort, see table 1). 5 phenocopies were Alport syndrome presenting clinically as FSGS/SRNS or vice versa. See Table 2 for the complete spectrum of phenocopies.

Conclusion: Phenocopies where found in every 5th solved case. A comprehensive picture of phenocopies is drawn illustrating the huge spectrum of clinical presentations of hereditary nephropathies and highlighting that exome sequencing is an appropriate diagnostic tool to account for this heterogeneity.

K.M. Riedhammer: None. M.C. Braunisch: None. R. Guenthner: None. M. Wagner: None. B. Uetz: None. B. Lange-Sperandio: None. J. Hoefele: None.

\section{C21.4}

Novel C-terminal $C U B N$ variants associate with chronic proteinuria and normal renal function
M. Bedin ${ }^{1}$, O. Boyer ${ }^{1,2}$, A. Servais ${ }^{3,1}$, L. Villoing-Gaudé ${ }^{1}$, O. Gribouval ${ }^{1}$, C. Bole ${ }^{1}$, F. Jabot-Hanin ${ }^{1}$, P. Nitschké ${ }^{1}$, C. Antignac ${ }^{1}$, M. Simons ${ }^{1}$

${ }^{1}$ INSERM U1163, Imagine Institute, Université Paris Descartes, Paris, France, ${ }^{2}$ Department of Pediatric Nephrology, Necker Hospital, Paris, France, ${ }^{3}$ Department of Nephrology, Necker Hospital, Paris, France

The loss of proteins into the urine (or proteinuria) is considered to be an important driver of kidney disease. The main reason is a defective glomerular filtration barrier allowing the passage of serum proteins, including albumin, into the urine. Largely overlooked, a failure of reabsorbing the filtered proteins in the proximal tubular segment can also lead to a mild tubular proteinuria, but it is unclear if it is harmful for the kidney. Here, we combine targeted and exome sequencing to explore the contribution of tubular genes to chronic subnephrotic proteinuria $(0,5-3 \mathrm{~g} / 24 \mathrm{~h})$. Altogether, we identified 24 patients with recessive mutations in the tubular uptake receptor cubilin $(C U B N)$. Meta-analyzing our in-house genetic database, we further identify a significant association between loss-of-function $C U B N$ mutations and proteinuric conditions. The tubular proteinuria in the $C U B N$ patients was atypical, lacking important tubular proteinuria markers and consisting of more than $50 \%$ albumin. Importantly, the renal function was normal, even in old patients, suggesting that the proteinuria is benign. All identified mutations localize to the C-terminal half of the protein, which is in contrast to the known N-terminal Imerslund-Gräsbeck mutations for CUBN featured by Vitamin B12 malabsorption in addition to the proteinuria. Some mutations have an allele frequency of $0.5 \%$ suggesting that this kind of proteinuria is not uncommon. This is supported by the gnomAD database showing a high prevalence of damaging $C U B N$ variants, mostly localizing to the C-terminal half. In summary, we identify C-terminal $C U B N$ mutations as a common but benign cause of chronic proteinuria.

M. Bedin: None. O. Boyer: None. A. Servais: None. L. Villoing-Gaudé: None. O. Gribouval: None. C. Bole: None. F. Jabot-Hanin: None. P. Nitschké: None. C. Antignac: None. M. Simons: None. 


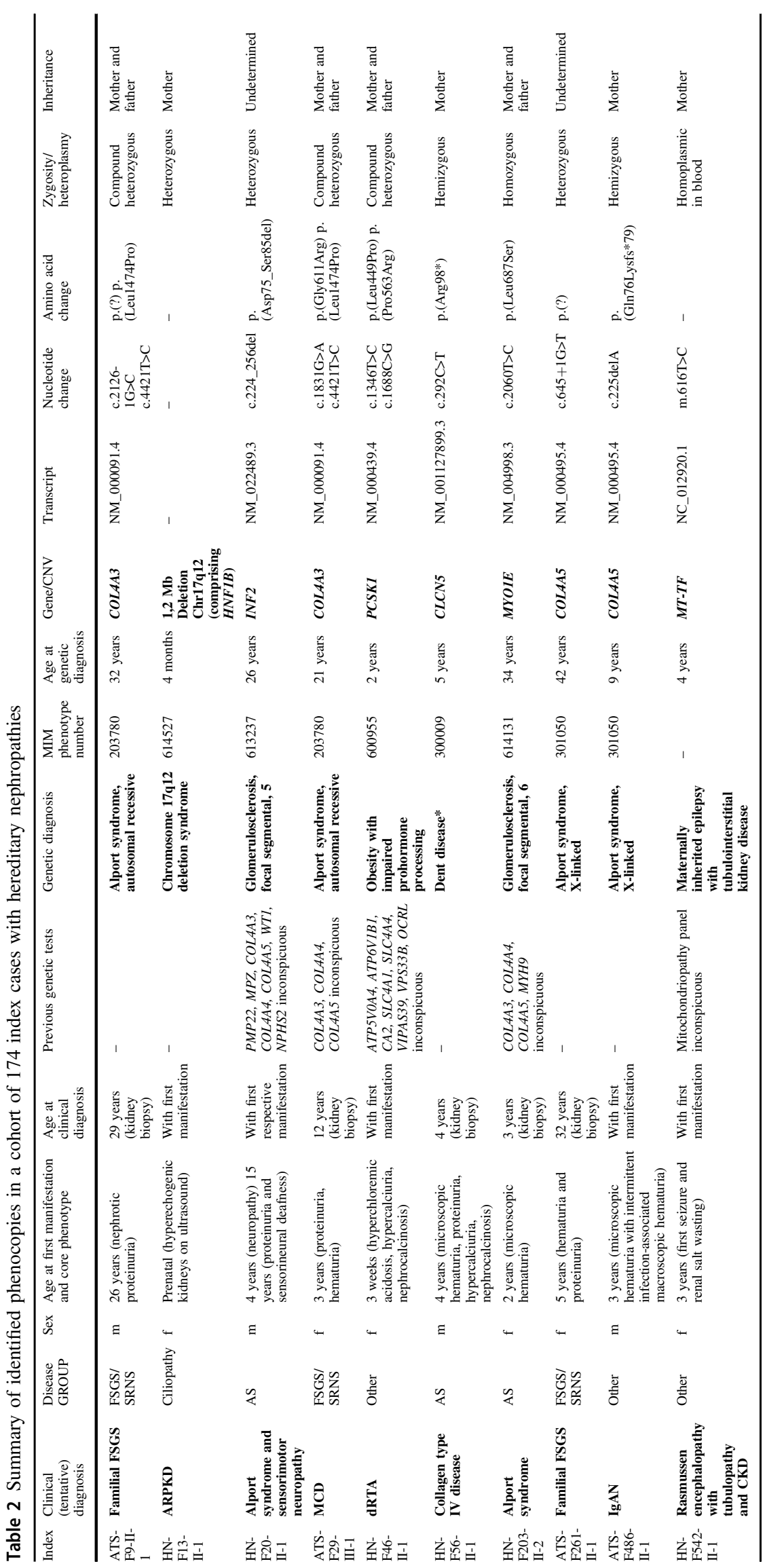




\section{C21.5}

Genome-wide association study of MRI liver iron content in 9,800 individuals yields new insights into its link with hepatic and extrahepatic diseases

H. R. Wilman ${ }^{1,2}$, C. A. Parisinos ${ }^{3}$, N. Atabaki-pasdar, M. Kelly ${ }^{2}$, E. L. Thomas ${ }^{1}$, S. Neubauer ${ }^{5}$, A. Hingorani, A. Mahajan ${ }^{5}$, R. Patel ${ }^{3}$, H. Hemingway,

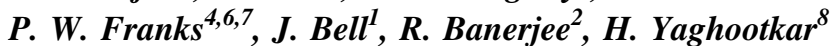

${ }^{1}$ University of Westminster, London, United Kingdom,

${ }^{2}$ Perspectum Diagnostics, Oxford, United Kingdom, ${ }^{3}$ University College London, London, United Kingdom, ${ }^{4}$ Lund University, Malmö, Sweden, ${ }^{5}$ University of Oxford, Oxford, United Kingdom, ${ }^{6}$ Umeå University, Umeå, Sweden, ${ }^{7}$ Harvard School of Public Health, Boston, MA, United States, ${ }^{8}$ University of Exeter, Exeter, United Kingdom

Excess liver iron is common and associates with hepatic and extrahepatic diseases. Genome-wide association studies (GWAS) liver iron, compared to circulating iron traits, permit detection of liver-specific susceptibility loci.

We performed the first GWAS of MRI determined liver iron in 8,289 individuals in UK Biobank and validated our results in 1,513 individuals from DIRECT. Liver iron was calculated from MRI data using LiverMultiScanTM. We used GEMMA, which corrects for relatedness and population structure, and adjusted our model for age, sex, BMI and genotyping array.

We identified three independent genetic variants (rs1800562 \& rs1799945 in HFE, rs855791 in TMPRSS6) associated with liver iron $(\mathrm{P}<5 \times 10-8)$. Both HFE and TMPRSS6 are involved in hepcidin production, the key hormone responsible for iron balance in the body. We estimated the heritability of liver iron to be $7.39 \%$. The most genetically correlated traits in LD score regression analysis were transferrin $(\mathrm{rG}=-0.78)$ and ferritin $(\mathrm{rG}=$ 1.24) with nominal significant correlation. In our Mendelian randomisation analysis, we found some evidence that higher central obesity, measured as waist-to-hip ratio, is casually associated with higher liver iron content (IVW $\mathrm{P}=0.003$ ). Phenome-wide association analysis demonstrates shared aetiology of elevated liver iron with high blood pressure, liver cirrhosis, extrahepatic malignancies, neuropsychiatric, endocrine and rheumatological conditions, and an inverse association with anaemias, lipidaemias and ischaemic heart disease.

Our study provides genetic evidence that mechanisms underlying excess liver iron are likely systemic not organ specific. Our data provides new insights into the biology of liver iron and its link to metabolic and non-metabolic disease outcomes.
H.R. Wilman: None. C.A. Parisinos: None. N. Atabaki-pasdar: None. M. Kelly: None. E.L. Thomas: None. S. Neubauer: None. A. Hingorani: None. A. Mahajan: None. R. Patel: None. H. Hemingway: None. P.W. Franks: None. J. Bell: None. R. Banerjee: None. H. Yaghootkar: None.

\section{C21.6}

Complex compound inheritance of lethal lung developmental disorders due to disruption of the TBXFGF pathway

J. A. Karolak', M. Vincent ${ }^{2}$, G. Deutsch ${ }^{3}$, T. Gambin ${ }^{4}$, B. Cognét $\hat{e}^{2}$ O. Pichon ${ }^{2}$, H. C. Mefford ${ }^{5}, J . N$. Dines ${ }^{5}$, M. Dishop ${ }^{6}$, D. Mowat ${ }^{7}$ A. J. Gifford ${ }^{8}$, A. F. Lee ${ }^{9}$, C. F. Boerkoel ${ }^{10}$, T. M. Bartell ${ }^{11}$, C. Ward-Melver ${ }^{12}$, T. Besnard ${ }^{2}$, F. Petit ${ }^{13}$, I. Bache ${ }^{14}$, Z. Tümer ${ }^{15}$, M. DenisMusquer $^{16}$, M. Joubert ${ }^{16}$, J. Martinovic ${ }^{17}$, E. Bieth ${ }^{18}$, N. Chassaing ${ }^{18}$, L. Devisme ${ }^{19}$, L. Pasquier ${ }^{20}$, M. Don $^{21}$, M. Orsaria ${ }^{22}$, S. Küry', S. Bézieau ${ }^{2}$, D. A. Scott', C. W. Brown ${ }^{23}$, F. Scaglia ${ }^{1}$, A. C. Tsai ${ }^{24}$, W. K. Chung ${ }^{25}$, G. Schauer ${ }^{26}$, C. L. Mercer ${ }^{27}$, A. van Haeringen ${ }^{28}$, Q. Liu ${ }^{1}$, E. Popek ${ }^{29}$, Z. H. Coban Akdemir', J. R. Lupski, P. Szafranski ${ }^{1}$, B. Isidor ${ }^{2}$, C. Le Caignec ${ }^{2}$, P. Stankiewicz ${ }^{1}$

${ }^{I}$ Dept of Molecular \& Human Genetics, Baylor College of Medicine, Houston, TX, United States, ${ }^{2}$ Service de Génétique Médicale, CHU Nantes, Nantes, France, ${ }^{3}$ Dept of Pathology, Seattle Children's Hospital, Seattle, WA, United States, ${ }^{4}$ Inst of Computer Science, Warsaw Univ of Technology, Warsaw, Poland, ${ }^{5}$ Dept of Pediatrics, Univ of Washington, Seattle, WA, United States, ${ }^{6}$ Pathology \& Laboratory Medicine, Phoenix Children's Hospital, Phoenix, AZ, United States, ${ }^{7}$ Centre for Clinical Genetics, Sydney Children's Hospital, Sydney, Australia, ${ }^{8}$ School of Women's \& Children's Health, Univ of NSW, Sydney, Australia, ${ }^{9}$ Dept of Pathology \& Laboratory Medicine, Univ of British Columbia, Vancouver, BC, Canada, ${ }^{10}$ Dept of Medical Genetics, Univ of British Columbia, Vancouver, BC, Canada, ${ }^{11}$ Dept of Genetics, Kaiser Permanente Sacramento Medical Center, Sacramento, CA, United States, ${ }^{12}$ Div of Medical Genetics, Akron Children's Hospital, Akron, $\mathrm{OH}$, United States, ${ }^{13}$ Service de Génétique Clinique, CHU Lille, Lille, France, ${ }^{14}$ Dept of Cellular \& Molecular Medicine, Univ of Copenhagen, Copenhagen, Denmark, ${ }^{15}$ Dept of Clinical Genetics, Copenhagen Univ Hospital, Rigshospitalet, Copenhagen, Denmark, ${ }^{16}$ Service d'anatomo-pathologie, CHU Nantes, Nantes, France, ${ }^{17}$ Unit of Fetal Pathology, Antoine Beclere Hospital, Paris, France, ${ }^{18}$ Service de génétique médicale, CHU Toulouse, Toulouse, France, ${ }^{19}$ Inst de Pathologie, CHU Lille, Lille, France, ${ }^{20}$ Service de génétique médicale, CHU Rennes, Rennes, France, ${ }^{21}$ Sant'Antonio General Hospital, 
Pediatric Care Unit, San Daniele del Friuli, Udine, Italy, ${ }^{22}$ Dept of Medical \& Biological Sciences, Univ of Udine, Udine, Italy, ${ }^{23}$ Dept of Pediatrics, Univ of Tennessee Health Science Center, Memphis, TN, United States, ${ }^{24}$ Dept of Pediatrics, The Children's Hospital, Univ of Colorado School of Medicine, Aurora, CO, United States, ${ }^{25}$ Dept of Pediatrics \& Medicine, Columbia Univ, New York, NY, United States, ${ }^{26}$ Dept of Pathology, Kaiser Permanente Oakland Medical Center, Oakland, CA, United States,

${ }^{27}$ Wessex Clinical Genetics Service, Univ Hospital Southampton NHS Foundation Trust, Princess Anne Hospital, Southampton, United Kingdom, ${ }^{28}$ Dept of Clinical Genetics, Leiden Univ Medical Center, Leiden, Netherlands, ${ }^{29}$ Dept of Pathology \& Immunology, Baylor College of Medicine, Houston, TX, United States

Introduction: Primary defects in lung branching morphogenesis, resulting in neonatal lethal pulmonary hypoplasias, are incompletely understood.

Materials and Methods: To elucidate the pathogenetics of human lung development, using WGS, ES, SNP and CGH arrays, Sanger sequencing, and qPCR, we studied samples obtained from deceased individuals with clinically and histopathologically diagnosed interstitial neonatal lung disorders: acinar dysplasia $(n=14)$, congenital alveolar dysplasia $(n=2)$, and other lethal lung hypoplasias $(n=$ 10). Seventeen family members and control individuals with $17 \mathrm{q} 23.1 \mathrm{q} 23.2(\mathrm{n}=13), 5 \mathrm{p} 12(\mathrm{n}=3)$, or an intragenic TBX4 $(\mathrm{n}=1)$ deletion but without a developmental lung disease were also investigated.

Results: We identified rare heterozygous copy-number variant deletions or single nucleotide variants (SNVs) involving TBX4 ( $\mathrm{n}=8$ and $\mathrm{n}=2$, respectively) or $F G F 10$ $(\mathrm{n}=2$ and $\mathrm{n}=2$, respectively) in $16 / 26(61 \%)$ affected individuals. In addition to $T B X 4$, the overlapping $\sim 2 \mathrm{Mb}$ recurrent and nonrecurrent deletions at 17q23.1q23.2 identified in seven individuals with lung hypoplasia also remove a lung-specific enhancer region. Individuals with coding variants involving either TBX4 or FGF10 also harbored at least one non-coding SNV in the predicted lung-specific enhancer region, which was absent in 13 control individuals with the overlapping deletions but without any structural lung anomalies.

Conclusion: The occurrence of rare coding variants involving TBX4 or FGF10 with the putative hypomorphic non-coding SNVs implies a complex compound inheritance of these pulmonary hypoplasias. Moreover, they support the importance of TBX4-FGF10-FGFR2 epithelialmesenchymal signaling in human lung organogenesis, and help to explain the histopathological continuum observed in these rare lethal developmental disorders of the lung (NIHR01HL137203).
J.A. Karolak: None. M. Vincent: None. G. Deutsch: None. T. Gambin: None. B. Cogné: None. O. Pichon: None. H.C. Mefford: None. J.N. Dines: None. M. Dishop: None. D. Mowat: None. A.J. Gifford: None. A.F. Lee: None. C.F. Boerkoel: None. T.M. Bartell: None. C. Ward-Melver: None. T. Besnard: None. F. Petit: None. I. Bache: None. Z. Tümer: None. M. Denis-Musquer: None. M. Joubert: None. J. Martinovic: None. E. Bieth: None. N. Chassaing: None. L. Devisme: None. L. Pasquier: None. M. Don: None. M. Orsaria: None. S. Küry: None. S. Bézieau: None. D.A. Scott: None. C.W. Brown: None. F. Scaglia: None. A.C. Tsai: None. W.K. Chung: None. G. Schauer: None. C.L. Mercer: None. A. van Haeringen: None. Q. Liu: None. E. Popek: None. Z.H. Coban Akdemir: None. J.R. Lupski: None. P. Szafranski: None. B. Isidor: None. C. Le Caignec: None. P. Stankiewicz: None.

C22 Ethical, policy and psychosocial aspects in genomics

C22.1

"To find out if it's genetic or not": Motivations, concerns and perceived impact of genome sequencing among young people
C. Lewis ${ }^{1,2}$, C. Patch ${ }^{3,4,5}$, M. Hill ${ }^{1,2}$, L. S. Chitty ${ }^{1,2}$, S. C. Sanderson ${ }^{1,6}$

${ }^{1}$ Great Ormond Street Hospital NHS Foundation Trust, London, United Kingdom, ${ }^{2}$ UCL Great Ormond Street Institute of Child Health, London, United Kingdom, ${ }^{3}$ Genomics England, London, United Kingdom, ${ }^{4}$ Florence Nightingale Faculty of Nursing and Midwifery, King's College London, London, United Kingdom, ${ }^{5}$ Counselling, Society and Ethics Research, Wellcome Genome Campus, Cambridge, United Kingdom, ${ }^{6}$ Institute of Health Informatics, University College London, London, United Kingdom

Background and methods: Until recently, many children with rare diseases were undiagnosed. Genome sequencing has been shown to significantly increase diagnostic yield and is therefore set to have a profound impact for children and young people with rare diseases. Although the current legal perspective is that children below a jurisdictionspecified age are unable to provide informed consent, young people, particularly adolescents, do frequently have the capacity to be actively involved in discussions about their health. We sought to characterise the attitudes of young people towards this technology by conducting 27 interviews with 11-18 year olds (excluding those with learning difficulties) taking part in the 100,000 Genomes Project. 
Results: Around two thirds of motivations cited by young people related to the potential benefits to themselves, including getting a diagnosis, "pinpointing" the gene causing the condition, understanding if the condition was inherited and might be passed on to future children, guiding treatment choices, ending further investigations and putting an end to the ongoing question of "why". Young people don't appear to be concerned about data security or companies having access to data, although some had concerns the results might reveal something "life threatening" and some were worried they would be "disappointed" if they did not get a result. Most were realistic that a result from genome sequencing would not necessarily lead to their condition being cured.

Conclusion: Young people are capable of grasping the potential benefits and limitations of genome sequencing and where possible should be actively engaged in the decisionmaking process.

C. Lewis: None. C. Patch: A. Employment (full or parttime); Modest; Secondment with Genomics England as Clinical Lead for Genetic Counselling since October 2016. M. Hill: None. L.S. Chitty: None. S.C. Sanderson: None.

\section{C22.2}

Genetic health professionals' experiences returning results from diagnostic genomic sequencing to patients

\section{F. Vears ${ }^{1,2,3}$, K. Sénécal ${ }^{4}$, P. Borry ${ }^{3}$}

${ }^{1}$ University of Melbourne, Parkville, Australia, ${ }^{2}$ Murdoch Children's Research Institute, Parkville, Australia, ${ }^{3}$ Centre for Biomedical Ethics and Law, KU Leuven, Leuven, Belgium, ${ }^{4}$ Centre of Genomics \& Policy, Mc Gill University, Montreal, QC, Canada

Despite widespread use of genomic sequencing (GS) in clinical care, there has been little exploration of actual experiences of genetic health professionals (GHPs) using these new technologies in their practice. To address this, semi-structured interviews were conducted with 31 clinical geneticists and genetic counsellors from 30 institutions across Europe, Australia and Canada to explore their experiences with returning results from GS, including positive and negative results, unsolicited findings (UF), and variants of uncertain significance (VUS), to patients.

GHPs remarked that patients' reactions to receiving positive results varied; some patients are relieved or appreciative at the identification of a genetic cause, whilst others express negative emotions, including frustration that finding an answer does not lead to a treatment. Participants discussed the importance of managing expectations in pretest counselling sessions to minimise disappointment. GHPs felt that even though some patients/families experienced mild distress, they generally cope quite well with receiving UF and appreciate receiving information about their increased risk. While many participants felt that most patients generally understand what a VUS means, a proportion of GHPs found VUS quite difficult to convey to patients and had concerns for their level of understanding. A considerable proportion of participants mentioned challenges associated with non-genetic HPs misinterpreting the significance of VUS and conveying this information to patients. This could have negative implications for patients and impacts on their own practice.

These results help us understand the challenges GHPs are experiencing in returning results from GS to patients, which has important implications for clinicians' training.

D.F. Vears: None. K. Sénécal: None. P. Borry: None.

C22.3

Parent experiences with ultra-rapid genomic sequencing in paediatric acute care

G. R. Brett ${ }^{1,2,3}$, M. Martyn ${ }^{3,2,4}$, M. de Silva ${ }^{1,2,5}$, K. Boggs ${ }^{5,6,7}$, A. Baxendale ${ }^{8,5}$, S. Borrie ${ }^{8,5}$, S. King-

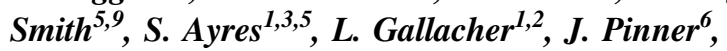
S. Sandaradura ${ }^{7}$, M. Wilson ${ }^{7}$, C. Barnett ${ }^{8}$, C. Patel ${ }^{10}$, A. Vasudevan ${ }^{11}$, E. Krzesinski ${ }^{12,13}$, S. Lunke ${ }^{1,2,5}$, Z. Stark ${ }^{1,2,5}$

${ }^{I}$ Victorian Clinical Genetics Services, Murdoch Children's Research Institute, Melbourne, Australia, ${ }^{2}$ University of Melbourne, Melbourne, Australia, ${ }^{3}$ Melbourne Genomics Health Alliance, Melbourne, Australia, ${ }^{4}$ Murdoch Children's Research Institute, Melbourne, Australia, ${ }^{5}$ Australian Genomics Health Alliance, (national), Australia, ${ }^{6}$ Sydney Children's Hospitals Network - Randwick, Sydney, Australia, ${ }^{7}$ Sydney Children's Hospitals Network - Westmead, Sydney, Australia, ${ }^{8}$ Paediatric and Reproductive Genetics Unit, South Australian Clinical Genetics Service, Adelaide, Australia, ${ }^{9}$ Centre for Cancer Biology, SA Pathology, University of South Australia, Adelaide, Australia, ${ }^{10}$ Genetic Health Queensland, Royal Brisbane and Women's Hospital, Brisbane, Australia, ${ }^{11}$ Royal Women's Hospital, Melbourne, Australia, ${ }^{12}$ Monash Children's Hospital, Melbourne, Australia, ${ }^{13}$ Department of Paediatrics, Monash University, Melbourne, Australia

Background: Emerging evidence that rapid turnaround times impact the clinical utility of genomic testing in acute paediatrics is driving widespread adoption. However, little is known about the experience that parents of critically unwell infants and children have during the testing process and beyond.

Methods: Participants were recruited as part of the Australian Genomics Acute Care study, a national rapid 
genomic diagnosis program for infants and children admitted to intensive care with suspected genetic conditions. Pre- and post-test counselling was provided by genetic health professionals. Over $95 \%$ of parents offered testing gave consent. Results were available within five days of sample receipt. Parents were surveyed $>12$ weeks after results return. We explored parental experiences with consent processes, perceived impact of testing on child health, relationships and reproductive decisions. This questionnaire included the Decision Regret, Short Form Genetic Counselling Outcomes and PedsQL Family Impact Module scales.

Results: From 21 respondents in the first six months $(\mathrm{RR}=54 \%)$, most felt they received enough information during pre-test $(\mathrm{n}=21,100 \%)$ and post-test $(\mathrm{n}=18,86 \%)$ counselling. No respondents reported decisional regret regarding testing. Perceptions varied about the benefits of rapid genomic sequencing for the child. The majority of respondents $(n=13,62 \%)$ were extremely concerned about the condition occurring in future children, regardless of their actual or self-perceived recurrence risk. Eight respondents (38\%) reported the test impacted their reproductive plans.

Importance: Understanding parental experiences, opinions, and the short and long term impacts on families will guide the design and delivery of rapid genomic diagnosis programs.

G.R. Brett: None. M. Martyn: None. M. de Silva: None. K. Boggs: None. A. Baxendale: None. S. Borrie: None. S. King-Smith: None. S. Ayres: None. L. Gallacher: None. J. Pinner: None. S. Sandaradura: None. M. Wilson: None. C. Barnett: None. C. Patel: None. A. Vasudevan: None. E. Krzesinski: None. S. Lunke: None. Z. Stark: None.

\section{C22.4}

The French FIND study (preliminary results). Psychological effects of actionable secondary findings obtained from exome sequencing in patients/families with undiagnosed rare diseases

\section{F. Houdayer ${ }^{1,2}$, A. Chassagne ${ }^{3,4}$, A. Pélissier ${ }^{4,5}$,}

C. Peyron ${ }^{4,5}$, S. Staraci ${ }^{2,6}$, D. Sanlaville ${ }^{1,7}$, P. Edery ${ }^{1,7}$,

M. Rossi ${ }^{8}$, G. Lesca ${ }^{I}$, A. Putoux ${ }^{I}$, L. Pons $^{I}$, A. Cadenes ${ }^{I}$, A. Baurand, C. Sawka ${ }^{9}$, G. Bertolone ${ }^{9}$, M. Spentchian ${ }^{10}$, M. Yousfi, D. Salvi ${ }^{5}$, E. Gautier ${ }^{9}$, A. Vitobello ${ }^{4}$, A. Dénommé-Pichon ${ }^{4}, A$. Faudet $^{10}$, B. Keren ${ }^{10}$, A. Labalme ${ }^{7}$, N. Chatron ${ }^{7}$, C. Abel ${ }^{1}$, S. Dupuis-Girod ${ }^{1}$, A. Poisson ${ }^{11}$, J. Buratti ${ }^{10}$, C. Mignot ${ }^{10}$, A. Afenjar ${ }^{10}$, S. Whalen ${ }^{10}$, P. Charles ${ }^{10}$, S. Heide ${ }^{10}$, L. Mouthon ${ }^{10}$, C. Thauvin-Robinet ${ }^{4,9}$, C. Philippe $e^{4,9}$, F. Tran MauThem $^{9}$, S. Moutton ${ }^{9}$, A. Sorlin', S. Nambot ${ }^{9}$, C. Binquet ${ }^{12}$, D. Héron ${ }^{10}$, M. Gargiulo $^{10,13}$, L. Faivre ${ }^{4,9}$
${ }^{1}$ Genetics Department, Reference Center for Developmental Disorders Sud East, HCL, Bron, France, ${ }^{2}$ Clinical Psychology Laboratory, Psychopathology, Psychoanalysis (EA4056), ED 261, Univ. Paris Descartes, Sorbonne Paris Cité, Paris, France, ${ }^{3}$ Sociology and anthropology laboratory LaSA, EA3189, CIC, INSERM 1431, Univ. de Bourgogne Franche-Comté, CHRU Besançon, Besançon, France, ${ }^{4}$ FHU TRANSLAD, GAD INSERM UMR 1231, Univ. de Bourgogne Franche-Comté, Dijon, Dijon, France, ${ }^{5}$ Economy laboratory of Dijon (LEDI), EA7467, Univ. de Bourgogne Franche-Comté, Dijon, France, ${ }^{6}$ Genetics Department, Reference Centre for Hereditary Cardiac Disorders, GH APHP, Paris, France, ${ }^{7}$ INSERM U1028, CNRS UMR5292, CRNL, GENDEV Team, Univ. Claude Bernard Lyon 1, Bron, France, ${ }^{8}$ Genetics Department, Reference Center for Developmental Disorders Sud East, HCL, Lyon, France, ${ }^{9}$ Genetics Department, Reference Center for Developmental Disorders, Dijon University Hospital, Dijon, France, ${ }^{10}$ Genetics Department GH APHP, Paris, France, ${ }^{11} \mathrm{CH}$ Le Vinatier, Bron, France, ${ }^{12}$ INSERM CIC-EC 1432, Dijon University Hospital, Dijon, France, ${ }^{13}$ Institute of Myology, GH APHP, Paris, France

Introduction: Access to active search for actionable secondary findings (SFs) in diagnostic practice is debated in Europe. The French FIND study has been set up to determine the short and longer-term benefits and risks according to the patients/families of returning SFs from diagnostic ES.

Materials and Methods: A mixed methodology implemented in three centers of expertise. This study offered to 330 patients with an undiagnosed developmental disorder the search for actionable a list of SFs from ES: SF1) predisposition to late-onset diseases (122 genes); SF2) carrier status of recessive or $\mathrm{X}$-linked diseases that could impact reproductive projects (114 genes); SF3) pharmacogenomic variants that could impact medical treatment (3 genes).

Results: Quantitative data show that $21 \%$ of patients did not wish to access SFs, citing anxiety, timing, or the study constraints. 94\% wished to access all SFs; $2 \%$ refused SF1, $1.6 \% \mathrm{SF} 2$ and $3 \% \mathrm{SF} 3$. Semi-directive interviews were scheduled with 15 patients whose SF results were positive, immediately after the results were returned. None of the patients expressed regrets regarding access to the SFs. Patients found it difficult to anticipate the psychological consequences of announcing an elevated risk of disease (SF1). Heterozygous carrier patients found it difficult to imagine the effects of sharing their results with their families (SF2). As for SF3, the medical benefits were not yet clear.

Conclusion: Literature showed that patients/families massively desire to know all SFs in hypothetical situations, 
contrasting with a $21 \%$ of refusal and with psychological effects in a real-life context.

F. Houdayer: None. A. Chassagne: None. A. Pélissier: None. C. Peyron: None. S. Staraci: None. D. Sanlaville: None. P. Edery: None. M. Rossi: None. G. Lesca: None. A. Putoux: None. L. Pons: None. A. Cadenes: None. A. Baurand: None. C. Sawka: None. G. Bertolone: None. M. Spentchian: None. M. Yousfi: None. D. Salvi: None. E. Gautier: None. A. Vitobello: None. A. DénomméPichon: None. A. Faudet: None. B. Keren: None. A. Labalme: None. N. Chatron: None. C. Abel: None. S. Dupuis-Girod: None. A. Poisson: None. J. Buratti: None. C. Mignot: None. A. Afenjar: None. S. Whalen: None. P. Charles: None. S. Heide: None. L. Mouthon: None. C. Thauvin-Robinet: None. C. Philippe: None. F. Tran Mau-Them: None. S. Moutton: None. A. Sorlin: None. S. Nambot: None. C. Binquet: None. D. Héron: None. M. Gargiulo: None. L. Faivre: None.

\section{C22.5}

Variant data sharing by clinical laboratories through public databases: consent, privacy and further contact for research policies

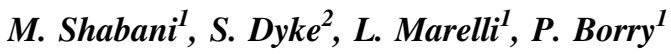 \\ ${ }^{1} \mathrm{KU}$ Leuven, Leuven, Belgium, ${ }^{2}$ McGill, Montreal, QC, Canada}

Access to detailed variant data is key to inform and verify the interpretation of genomic data. Clinical laboratories can play a significant role in sharing patients' data through public variant databases. In order to facilitate data sharing, various public databases such as ClinVar (in collaboration with ClinGen) and DECIPHER have been established, which accept data submission from laboratories, clinicians, researchers and patients. Despite clear benefits to sharing, questions may arise about the adequate form of consent to be obtained from patients when sharing data from their clinical tests through public databases. In particular, how much information should be communicated to the patients regarding data sharing? Should patients have the option to opt-out? Under what conditions could laboratories share more detailed information (e.g. supporting individual-level data) about the patients than simply the aggregate variantlevel classifications? In order to address these questions, we conducted a content analysis of the relevant consent policies of the major public databases and of the consent forms of clinical laboratories who share variant data. Our study showed that despite the guidance provided by the data repositories, the approaches towards consent vary across the studied laboratories. Moreover, we analyzed the information provided in the consent forms regarding the risks of re- identification and the potential for further contact for research. We concluded with providing further analysis on the adequacy of the current consent and privacy policies of the laboratories and databases in the view of relevant data protection regulations.

M. Shabani: None. S. Dyke: None. L. Marelli: None. P. Borry: None.

\section{C22.6}

Are requirements to deposit data in research repositories compatible with the GDPR?

D. Mascalzoni ${ }^{1,2}$, H. B. Bentzen ${ }^{3}$, I. Budin-Ljøsne ${ }^{4}$, L. A. Bygrave ${ }^{3}$, J. Bell ${ }^{5,6}$, E. S. Dove ${ }^{7}$, C. Fuchsberger ${ }^{1}$, K. Hveem $^{8}$, M. T. Mayrhofer ${ }^{9}$, V. Meraviglia ${ }^{\text {, }}$, D. R. O'Brien ${ }^{10}$, C. Pattaro' ${ }^{1}$ P. P. Pramstaller ${ }^{1}$, V. Rakic ${ }^{11}$, A. Rossini ${ }^{1}$, M. Shabani ${ }^{12,13}$, D. J. B. Svantesson ${ }^{14}$, M. Tomasi ${ }^{15,16}$, L. Ursin ${ }^{8}$, M. Wjst ${ }^{17,18}$, J. Kaye $e^{5,6}$

${ }^{1}$ Eurac Research, Bolzano, Italy, ${ }^{2}$ Uppsala University, Uppsala, Sweden, ${ }^{3}$ University of Oslo, Oslo, Norway, ${ }^{4}$ Norwegian Institute of Public Health, Oslo, Norway, ${ }^{5}$ University of Oxford, Oxford, United Kingdom, ${ }^{6}$ University of Melbourne, Melbourne, Australia, ${ }^{7}$ University of Edinburgh, Edinburgh, United Kingdom, ${ }^{8}$ Norwegian University of Science and Technology, Trondheim, Norway, ${ }^{9}$ BBMRI-ERIC, Graz, Austria, ${ }^{10}$ Harvard University, Boston, MA, United States, ${ }^{11}$ University of Belgrade, Belgrade, Serbia, ${ }^{12}$ KU Leuven, Leuven, Belgium, ${ }^{13}$ Leuven Institute for Human Genomics and Society, Leuven, Belgium, ${ }^{14}$ Bond University, Gold Coast, Australia, ${ }^{15}$ University of Trento, Trento, Italy, ${ }^{16}$ Free University of Bozen-Bolzano, Bolzano, Italy, ${ }^{17}$ Helmholtz Zentrum München, Munich, Germany, ${ }^{18}$ Technical University Munich, Munich, Germany

Increasingly, journals' and funding bodies' policies require researchers to deposit individual level data in research repositories. The aim is noble: to increase data sharing which facilitates reproducibility and new discoveries. However, data deposit requirements may conflict with ethical and legal obligations, including the EU General Data Protection Regulation (GDPR). The main obstacles relate to i) loss of control over further processing; ii) realization of rights and obligations; iii) national derogations; iv) jurisdictional issues; and v) accountability. These ethical and legal challenges of depositing individual level genetic data in research repositories such as dbGaP will be described and illustrated with practical real-life examples from some of Europe's largest biobanks. The framework of research data deposition, if not adapted, could lead to the unwanted consequence of researchers complying with the law facing difficulties publishing their work, irrespective of its scientific qualities. Such de facto inequities in the 
scientific evaluation process, may endanger scientific excellence and evidence-based medicine. In addition to bringing current repositories in compliance, further potential solutions to optimize responsible data sharing will be described, including i) dynamic consent, ii) data tagging, and iii) federated data sharing. The policy paper springs out from work done in the COST Action CHIP ME, an EU funded European research network of researchers working on ethics and law related to genomics. Additionally, legal researchers from the US, Canada, and Australia, the leaders of some of Europe's largest biobanks and a representative from the European biobank network BBMRI-ERIC have contributed to the work.

D. Mascalzoni: None. H.B. Bentzen: None. I. BudinLjøsne: None. L.A. Bygrave: None. J. Bell: None. E.S. Dove: None. C. Fuchsberger: None. K. Hveem: None. M. T. Mayrhofer: None. V. Meraviglia: None. D.R. O Brien: None. C. Pattaro: None. P.P. Pramstaller: None. V. Rakić: None. A. Rossini: None. M. Shabani: None. D.J. B. Svantesson: None. M. Tomasi: None. L. Ursin: None. M. Wjst: None. J. Kaye: None.

\section{C24 Mosaicisms}

C24.1

Analysis of Mosaicism for Sequence and Copy Number Variants in a Broad Diversity of Hereditary Disorders in a Large Clinical Cohort

\section{Pineda Alvarez, R. Truty, T. Hambruch, C. Kautzer, M. Kennemer, K. Kosheleva, V. Kulkarni, J. Rhees, A. Stafford, R. Nussbaum, S. Aradhya}

\section{Invitae, San Francisco, CA, United States}

Analysis of mosaic variants in hereditary diseases can provide insight into analytic requirements for detecting such variants and their clinical implications. We validated a nextgeneration sequencing method with high depth of coverage to detect mosaic single-nucleotide variants (SNVs), indels, and copy number variants (CNVs) using Genome-in-aBottle (GiaB) sample dilution series. In a clinical cohort of 421,220 individuals, we identified 1,799 mosaic SNVs and small indels and 84 mosaic CNVs across 245 genes, corresponding to $0.6 \%$ of reported variants. This represents an incidence of $0.9 \%$ among individuals who had at least one reported variant. Approximately $40 \%$ of mosaic variants were classified as likely or definitively pathogenic and these contributed to $1.1 \%$ of definitive diagnoses in this cohort. Nearly $80 \%$ of clinically significant mosaic variants were associated with autosomal dominant disorders. There were nearly twice as many cancer-related genes with mosaic variants relative to those associated with pediatric, neurological, or cardiovascular disorders. Later onset diseases were associated with lower levels of mosaicism in affected individuals compared to diseases with early onset $(\mathrm{p}=$ 0.002; Wilcoxon signed rank). In relation to hereditary cancer, individuals with mosaic variants were considerably older than those without mosaic variants $\left(\mathrm{p}=6.5 \times 10^{-48}\right.$; Student's t-test). Finally, among individuals who had a disease with pathognomonic features, those with mosaic variants showed non-classical phenotypes compared to those with non-mosaic variants $(\mathrm{p}=0.0009$; Fisher's exact test). These exhaustive analyses provide insight into mosaicism in Mendelian disease genes and inform expectations for diagnostic testing, clinical correlations, and recurrence risk.

D. Pineda Alvarez: None. R. Truty: A. Employment (full or part-time); Significant; Invitae. T. Hambruch: None. C. Kautzer: A. Employment (full or part-time); Significant; Invitae. M. Kennemer: A. Employment (full or part-time); Significant; Invitae. K. Kosheleva: None. V. Kulkarni: None. J. Rhees: A. Employment (full or parttime); Significant; Invitae. A. Stafford: A. Employment (full or part-time); Significant; Invitae. R. Nussbaum: A. Employment (full or part-time); Significant; Invitae. S. Aradhya: None.

\section{C24.2}

Uniparental disomy in the Rare Disease Programme of the UK's 100,000 Genomes Project

K. R. Smith ${ }^{1,2}$, M. Bleda $^{2}$, D. Kasperaviciute ${ }^{1,2}$, K. Ibanez $^{2}$, A. Rueda Martin', E. Thomas ${ }^{2}, E_{\text {Baple }}^{2}$, A. Tucci', M. J. Caulfield ${ }^{1,2}$, A. Rendon ${ }^{3,2}$

${ }^{1}$ Queen Mary University of London, London, United Kingdom, ${ }^{2}$ Genomics England, London, United Kingdom, ${ }^{3}$ University of Cambridge, Cambridge, United Kingdom

Uniparental disomy (UPD) occurs when one parent contributes two homologous copies of a particular chromosomal segment and the other parent none. UPD can cause genetic disorders by disrupting imprinting or homozygosing heterozygous disease alleles. We present data on chromosomal-scale UPD detection using whole genome sequence (WGS) data from parent-child pairs in the Rare Disease programme of the UK's 100,000 Genomes Project. Routine quality control flags duos with atypical identity by descent sharing patterns or elevated Mendelian inconsistency rates; signatures typical of UPD are reviewed to confirm and characterise the event.

We have currently identified 42 UPD events (27 maternal, 14 paternal) at a rate of $0.34 \%$. Both parents were sequenced for 37 events. Thirty-eight involved whole 
chromosomes while four were partial. Only four heterodisomy events were detected, whereas isodisomy and mixed iso-and-heterodisomy occurred at similar frequencies. For 38 families UPD was detected in the only affected family member so could plausibly be the cause of the rare disorder. Thirteen events occurred on five chromosome/parent combinations where UPD can cause imprinting disorders, enabling potential diagnoses invisible to small variant pipelines.

Clinical interpretation has been completed for $9 / 42$ families. Four events were confirmed from four attempts and three cases reported as solved. All three cases resulted from UPD homozygosing the disease allele $(n=2)$ or disrupting imprinting $(\mathrm{n}=1)$. Our work shows that UPD can be detected using WGS from trios or duos where the undertransmitting parent is sequenced. UPD detection can lead to additional diagnoses in rare disease sequencing programmes.

K.R. Smith: None. M. Bleda: None. D. Kasperaviciute: None. K. Ibanez: None. A. Rueda Martin: None. E. Thomas: None. E. Baple: None. A. Tucci: None. M.J. Caulfield: None. A. Rendon: None.

\section{C24.3}

Somatic mutation cell lineage analysis reveals progressive clonal determination in human embryo

S. Bizzotto' ${ }^{1}$ J. Ganz' ${ }^{2}$ Y. Dou ${ }^{3}$, R. N. Doan', E. Maury,

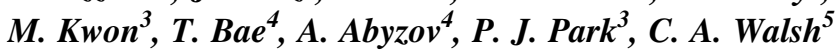

${ }^{1}$ Boston Children's Hospital, Department of Genetics and Genomics, Manton Center for Orphan Disease, Boston, MA, United States, ${ }^{2}$ Boston Children's Hospital, Department of Genetics and Genomics, Boston, MA, United States, ${ }^{3}$ Harvard Medical School, Department of Biomedical Informatics, Boston, MA, United States, ${ }^{4}$ Mayo Clinic, Department of Health Sciences Research, Rochester, MN, United States, ${ }^{5}$ Boston Children's Hospital, Department of Genetics and Genomics, Howard Hughes Medical Institute, Boston, MA, United States

Little is known about embryogenesis and organ specification in humans due to the inherent difficulty of studying these processes directly in vivo. However, next-generation sequencing (NGS) techniques are providing useful and unprecedented tools that can be applied to reveal unexplored mechanisms. We combine single cell DNA sequencing and high coverage (250X whole genome) DNA sequencing in three normal individuals to identify somatic single nucleotide variants (sSNVs) that arose during embryogenesis, and use them as forensic barcodes for cell lineage tracing in postmortem adult humans. Analysis of 1,000 single neurons and non-neuronal cells isolated from a single individual generates a dense lineage tree that allows us to obtain the clonal structure of developmental variants for this individual. Variant deep targeted-sequencing on bulk DNA extracted from serial coronal sections of an entire human cerebral hemisphere reveals gradual, clonal segregation across the cortex with a persistent polyclonal end result, while analysis of 22 other organs and tissues reveals a mosaic architecture of organs deriving from distinct germ layers. Inferring the developmental cell stages of variant occurrence discloses key aspects of human embryogenesis and numbers of effective progenitor cells at different steps such as blastocyst formation and gastrulation. We show that sSNVs permit cell lineage maps of any human at postmortem, of density and breadth limited only by sequencing depth, with clonal analyses directly revealing key aspects of human embryonic development. Grant references: NINDS R01NS032457, Manton Center for Orphan Disease Research Fellowship

S. Bizzotto: None. J. Ganz: None. Y. Dou: None. R.N. Doan: None. E. Maury: None. M. Kwon: None. T. Bae: None. A. Abyzov: None. P.J. Park: None. C.A. Walsh: None.

\section{C24.4}

Basal and mutagen-driven somatic mutagenesis shape the genome of healthy human cells

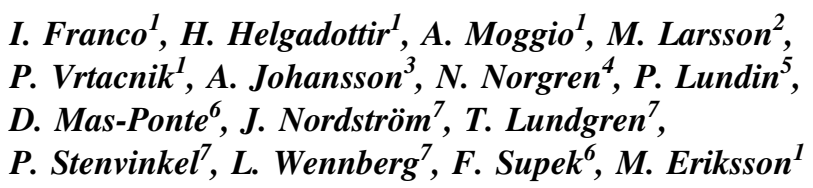

${ }^{1}$ Karolinska Institutet, HUDDINGE, Sweden, ${ }^{2}$ Science for Life Laboratories, Linköping, Sweden, ${ }^{3}$ Science for Life Laboratories, Uppsala, Sweden, ${ }^{4}$ Science for Life Laboratories, Umeå, Sweden, ${ }^{5}$ Science for Life Laboratories, Stockholm, Sweden, ${ }^{6}$ Institute for Research in Biomedicine (IRBB), Barcelona, Spain, ${ }^{7}$ Karolinska University Hospital, HUDDINGE, Sweden

Analyses of somatic mutations in healthy tissues can identify mutagens active in specific organs. We generated WGS data from freshly isolated single cells clonally expanded in vitro $(\mathrm{N}=69)$ and created a genetic atlas of somatic mutations in healthy human tissues (kidney, skin, subcutaneous fat and visceral fat). Multi-organ biopsies from the same individual allowed well-controlled inspection of tissue-specific differences, while donors of two age groups (30s and 60s) provided data on the effects of aging. This analysis, complemented with a meta-analysis of somatic mutations from healthy $(\mathrm{N}=161)$ and tissuematched cancer genomes $(\mathrm{N}=192)$, showed a stereotyped mutational pattern across multiple cell types. Conversely, 
cells derived from the kidney tubules grouped into two separate populations showing either the stereotyped or a unique mutation pattern with high mutation burden, high signatures SBS5 and SBS40 and enrichment of mutations in transcribed genes. These findings challenged the concept of a tissue-specific mutation accumulation and instead supported the idea that specific mutagenic processes shape the genome of distinct cell types. A number of cells, including fat, skeletal muscle, kidney and blood progenitors, showed no signs of exposure to external mutagens, but a common "basal mutagenesis" that we have characterised. The DNArepair machinery counteracted the basal mutagenesis but was less efficient with aging and continuous mutagen exposure. Complete understanding of the mutagenic forces active in healthy cells will be important for the prevention of mutations and consequent cancer development.

Supported by Swedish Research Council; Center for Innovative Medicine; Hagelen, Jeanssons and Osterman Foundations; Svenska Läkaresällskapet; Riksbanken Jubileumsfond.

I. Franco: None. H. Helgadottir: None. A. Moggio: None. M. Larsson: None. P. Vrtacnik: None. A. Johansson: None. N. Norgren: None. P. Lundin: None. D. Mas-Ponte: None. J. Nordström: None. T. Lundgren: None. P. Stenvinkel: None. L. Wennberg: None. F. Supek: None. M. Eriksson: None.

\section{C24.5}

Genetic basis of mosaic pigmentary disorders of the skin and how to detect them: the M.U.S.T.A.R.D. cohort's experience

A. Sorlin ${ }^{1,2,3}$, V. Carmignac ${ }^{2,3}$, P. Kuentz ${ }^{3,4}, \hat{E}$. Tisserant $^{2,3}$, Y. Duffourd ${ }^{2,3}$, M.U.S.T.A.R.D. cohort's investigators, J. Rivière (2,3 $^{2, \text { P. Callier }}{ }^{3,5}$, C. Philippe ${ }^{2,3}$, C. Thauvin ${ }^{1,2,3}$, L. Faivre ${ }^{1,2,3}$, P. Vabres ${ }^{3,6,7}$

${ }^{1}$ Centre de Génétique, CHU Dijon Bourgogne, Dijon, France, ${ }^{2}$ Unité Fonctionnelle Innovation en Diagnostic génomique des maladies rares, FHU-TRANSLAD, CHU Dijon Bourgogne, Dijon, France, ${ }^{3}$ INSERM 1231, Génétique des Anomalies du Développement, Université Bourgogne Franche-Comté, Dijon, France, ${ }^{4}$ Génétique Biologique Histologie, CHRU de Besançon, Besançon, France, ${ }^{5}$ Laboratoire de génétique moléculaire et de Cytogénétique, Plateau Technique de Biologie, CHU Dijon Bourgogne, Dijon, France, ${ }^{6}$ Centre de référence MAGEC Mosaïques, CHU Dijon Bourgogne, Dijon, France, ${ }^{7}$ Service de Dermatologie, CHU Dijon Bourgogne, Dijon, France

Introduction: Mosaic cutaneous dyschromia (MCD: segmental hypopigmentation, such as Hypomelanosis of Ito, and/or hyperpigmentation) is a rare symptom strongly evocative of an underlying genetic mosaicism. These postzygotic events are challenging for conventional diagnostic tools, as they concern only a fraction of cells often confined to the affected tissue. Thus, genetic basis of MCD still remained poorly understood.

Materials and Methods: The M.U.S.T.A.R.D. cohort gathers DNA from skin biopsies of patients with development anomalies including MCD and their parents, phenotyped at the reference center for rare diseases MAGEC (Dijon), and referred to either targeted ultra-deep sequencing $(60,000 \mathrm{X})$ of candidate genes, or trio exome sequencing (ES) at 200X. Data are analysed with a tailored pipeline, allowing detection of low-rate nucleotidic variations or chromosomal events (mosaic CNV, mosaic uniparental disomy (UPD)).

Results: From 2014 to 2019, 122 patients were included. ES was performed for 42, with identification of mosaic SNV in 5 candidate genes in 10 patients, and mosaic CNV (monosomy/trisomy/tetrasomy/triploidy) or UPD in 9. Based on the recognisable clinical presentations therefore identified, we selected 40 patients for targeted sequencing of at least one candidate gene, with a confirmed mosaic SNV in 14, and a global diagnostic yield of $40 \%$.

Conclusion: This work highlights the importance of a versatile bioinformatic approach combined to a clinical expertise, to decipher the chromosomal and molecular aetiologies of developmental anomalies with MCD. It also pinpoints common pathogenesis pathways, which will help enhancing our understanding of MCD, and may ultimately result in novel therapeutic opportunities.

A. Sorlin: None. V. Carmignac: None. P. Kuentz: None. É. Tisserant: None. Y. Duffourd: None. J. Rivière: None. P. Callier: None. C. Philippe: None. C. Thauvin: None. L. Faivre: None. P. Vabres: None.

C24.6

The Hutchinson-Gilford progeria syndrome mutation is a somatic mutation in chronic kidney disease

M. Eriksson ${ }^{1}$, H. T. Helgadottir ${ }^{1}$, N. Viceconte ${ }^{1}$, A. Witasp ${ }^{2}$, E. Wallén Arzt ${ }^{1}$, A. Sola Carvajal, G. Revechon ${ }^{1}$, A. Thorell ${ }^{3}$, D. McGuinness ${ }^{4}$, P. G. Shiels ${ }^{4}$, A. Wernerson ${ }^{2}$, P. Stenvinkel ${ }^{2}$

${ }^{1}$ Karolinska Institutet, Dept of Biosciences and Nutrition, Huddinge, Sweden, ${ }^{2}$ Karolinska Institutet, Department of Clinical Science, Intervention and Technology, Division of Renal Medicine, Stockholm, Sweden, ${ }^{3}$ Karolinska Institutet, Department of Clinical Sciences, Danderyds Hospital, Department of Surgery, Ersta Hospital, Stockholm, Sweden, ${ }^{4}$ University of Glasgow, Institute of Cancer Sciences, Glasgow, United Kingdom 
Introduction: Premature vascular aging is a hallmark of chronic kidney disease (CKD). The phenotype include arteriosclerosis, loss of vascular smooth muscle cells, calcification and increased arterial stiffness. A similar vascular phenotype is also observed in children with the premature aging disease Hutchinson-Gilford progeria syndrome (HGPS). HGPS is caused by a single-nucleotide mutation in the LMNA gene, c. $1824 \mathrm{C}>\mathrm{T}$. This mutation leads to increased production of a truncated protein, progerin, and dysregulation of cell functions. Progerin has been found at low levels in skin and arteries from nonprogeroid individuals, but the underlaying mechanism is unknown.

Materials and Methods: Here we analyzed the presence of progerin in epigastric arteries obtained from CKD stage 5 patients (GFR $<15 \mathrm{ml} / \mathrm{min}$ ) and controls, and its contribution to premature vascular aging. Somatic mutation analysis included DNA extracted from progerin stained sections from CKD arteries $(\mathrm{n}=27)$, using digital droplet PCR.

Results: Immunofluorescence showed progerin positive cells in $70 \%$ of the arteries $(46 \pm 2 \mathrm{yrs}, \mathrm{n}=40)$. Progerin was detected in up to $7.4 \%$ of cells. The number of progerin positive cells correlated with number of years with the disease $(P=0.03, \mathrm{r}=0.34)$. The arteries with higher frequency of progerin had an increased activation of DNA repair $(P=0.005, \mathrm{r}=0.63)$. Somatic mutation analysis identified the LMNA c.1824C $>\mathrm{T}$ mutation in blood and arteries.

Conclusion: Our results suggest that rare somatic mutations may become a risk when increased tissue regeneration is induced, and provide novel mechanistic insight to the underlying phenotype of premature vascular aging.

M. Eriksson: None. H.T. Helgadottir: None. N. Viceconte: None. A. Witasp: None. E. Wallén Arzt: None. A. Sola Carvajal: None. G. Revechon: None. A. Thorell: None. D. McGuinness: None. P.G. Shiels: None. A. Wernerson: None. P. Stenvinkel: None.

\section{C25 Bioinformatics and multiomics}

\section{C25.1}

A transcriptome-wide Mendelian randomization study to uncover tissue-dependent regulatory mechanisms across the human phenome

\section{T. G. Richardson, G. Hemani, T. R. Gaunt, C. L. Relton, G. Davey Smith}

MRC Integrative Epidemiology Unit, Bristol, United Kingdom
Background: Developing insight into tissue-specific transcriptional mechanisms can help improve our understanding of how genetic variants exert their effects on complex traits and disease. By applying the principles of Mendelian randomization, we have undertaken a systematic analysis to evaluate transcriptome-wide associations between gene expression and 395 complex traits across 48 different tissue types.

Results: Overall, we identified 59,810 gene-trait associations in at least 1 tissue type based on multiple testing and genetic colocalization corrections. These results indicate that genetic effects expressed more ubiquitously across different tissues are more likely to be pleiotropic (i.e. associated with multiple diverse phenotypes). We have also explored these results in detail with a series of analyses that highlight tissue-dependent effects. For instance, TPO, $N R 3 C 2$ and SPATA13 expression was only associated with thyroid disease in thyroid tissue, whereas $F B N 2$ was associated with both cardiovascular and lung function traits but only when analysed in heart and lung tissue respectively.

We also demonstrate that phenome-wide investigations of our results can be valuable for drug validation purposes. Finally, we have evaluated the tissue-dependency of findings from genome-wide association studies (GWAS). This suggested that integrating tissue-specific data with GWAS results can help elucidate the causal genes and tissues responsible for effects, as well uncover putative novel findings.

Conclusion: The atlas of tissue-dependent associations we have constructed should prove extremely insightful to future studies investigating the genetic determinants of complex disease. Follow-up analyses in this study are merely a guide for future research and similar evaluations can be undertaken at http://mrcieu.mrsoftware.org/Tissue MR_atlas/.

T.G. Richardson: None. G. Hemani: None. T.R. Gaunt: None. C.L. Relton: None. G. Davey Smith: None.

\section{C25.2}

Multivariate GWAS of inflammatory markers reveals novel disease associations

S. E. Ruotsalainen ${ }^{1}$, J. Partanen ${ }^{1}$, I. Surakka ${ }^{1,2}$,

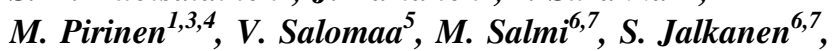
M. J. Daly ${ }^{1,8}$, S. Ripatti ${ }^{1,3,8}$, J. Koskela ${ }^{1}$

${ }^{1}$ Institute for Molecular Medicine Finland, University of Helsinki, Helsinki, Finland, ${ }^{2}$ Department of Internal Medicine, University of Michigan, Ann Arbor, MI, United States, ${ }^{3}$ Department of Public Health, Faculty of Medicine, University of Helsinki, Helsinki, Finland, ${ }^{4}$ Department of Mathematics and Statistics, University of Helsinki, Helsinki, Finland, ${ }^{5}$ National Institute for Health and Welfare, 
Helsinki, Finland, ${ }^{6}$ Medicity Research Laboratory, University of Turku, Turku, Finland, ${ }^{7}$ Institute of Biomedicine, University of Turku, Turku, Finland, ${ }^{8}$ Broad Institute of MIT and Harvard, Cambridge, MA, United States

Multivariate analysis of correlated traits is known to increase power compared to univariate analysis, although it remains highly underutilized in GWASs to date. Since many inflammatory markers are both correlated and associated with multiple diseases, we aimed to leverage correlated biomarkers in discovery of disease-related genetic variants.

We used data from the population-based FINRISK Study of up to 26,705 Finns and imputed genotypes to $13 \mathrm{M}$ variants. We analyzed 66 cardiovascular or immunologic relevant traits, including anthropometric and biomarkers such as blood lipids and inflammatory markers. Out of the 66 traits, hierarchical clustering identified one highly correlated cluster, comprised of 12 inflammatory markers mainly interleukins and growth factors. We then performed multivariate GWAS on this cluster using metaCCA, a summary-statistic based multivariate method applying Canonical Correlation Analysis.

Using multivariate method, we observed 89 genome-wide significant loci, a substantial increase over the full set of 28 that were genome-wide significant in one or more of the 12 traits. To illustrate, protective variant for pulmonary embolism rs4656185 in F5 was genome-wide significant only using multivariate method (multivariate $\mathrm{p}$-value $=$ 7.9e-20, smallest univariate $\mathrm{p}$-value $=9.9 \mathrm{e}-4$ ). In addition, out of 89 genome-wide significant loci 13 were reported to associate with at least one of the traits in the cluster, 7 with other cytokines or leukocyte counts and 19 with at least one disease-endpoint $(\mathrm{p}<1 \mathrm{e}-5)$ in GWAS catalog, UKBB or FinnGen. In conclusion, our study shows how multivariate method provides a remarkable boost in power for locus discovery, but also emphasizes the advantage of large biobank-based phenome-wide screening.

S.E. Ruotsalainen: None. J. Partanen: None. I. Surakka: None. M. Pirinen: None. V. Salomaa: None. M. Salmi: None. S. Jalkanen: None. M.J. Daly: None. S. Ripatti: None. J. Koskela: None.

\section{C25.3}

Analysis of genetic variants through aggregation of homologous human protein domains via MetaDome strongly improves diagnostic prediction of missense variants

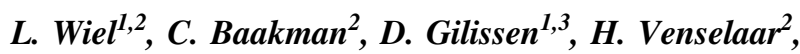
J. A. Veltman ${ }^{4,5}$, G. Vriend $^{2}$, C. Gilissen ${ }^{1}$
${ }^{1}$ Department of Human Genetics, Radboud Institute for Molecular Life Sciences, Radboud University Medical Center, Nijmegen, Netherlands, ${ }^{2}$ Centre for Molecular and Biomolecular Informatics, Radboud Institute for Molecular Life Sciences, Radboud University Medical Center, Nijmegen, Netherlands, ${ }^{3}$ Bio-informatica, HAN University of Applied Sciences, Nijmegen, Netherlands, ${ }^{4}$ Department of Human Genetics, Donders Institute for Brain, Cognition and Behaviour, Radboud University Medical Center, Nijmegen, Netherlands, ${ }^{5}$ Institute of Genetic Medicine, International Centre for Life, Newcastle University, Newcastle upon Tyne, United Kingdom

We developed a novel concept based on protein domain homology in the human genome to improve variant interpretation. For this purpose we mapped populationbased variation from the Exome Aggregation Consortium (ExAC) and disease-causing mutations from the Human Gene Mutation Database (HGMD) onto 5,250 Pfam protein domain families. We then aggregated population-based and disease-causing variants across 30,853 homologous protein domains into 2,750 meta-domains.

We find that genetic tolerance is consistent across $97 \%$ of domain homologues $(\mathrm{p}<0.05$, Bonferroni corrected), and that patterns of genetic tolerance faithfully mimic patterns of evolutionary conservation (Pearson 0.97, p-value $<1$ e308 ). Interestingly, we find that 2,201 of the aggregated domain positions are not evolutionary conserved, but highly intolerant to population-based variation and contain at least one disease-causing missense variant. An informative example concerns domain positions 17 and 21 in the "EGFlike domain" (PF00008). These positions are not conserved but are depleted of population-based variation in 60 genes and additionally cause disease in the NOTCH3, JAG1, and $C R B 2$ genes. Furthermore, we find that the presence of disease-causing missense variants at aggregated homologous domain positions is often paired with the absence of population variation and vice versa.

We realized that this information could be of great benefit to the genetic community and therefore developed MetaDome; a fast and easy-to-use web server that visualizes meta-domain information and gene-wide profiles of genetic tolerance. MetaDome allows to easily investigate variants of interest for the presence or absence of variation at corresponding positions within homologous domains and is freely accessible at https://stuart.radboudumc.nl/metadome.

L. Wiel: None. C. Baakman: None. D. Gilissen: None. H. Venselaar: None. J.A. Veltman: None. G. Vriend: None. C. Gilissen: None.

\section{C25.4}

reg2gene: predicting enhancer-gene associations using ensemble learning approaches 


\section{Patarcic, V. Franke, A. Akalin}

\section{BIMSB, Berlin, Germany}

Modelling procedures such as JEME (Cao et al. 2017), FOCS (Hait et al. 2018), GeneHancer (Fishillevich et al. 2017), and EnhancerAtlas(Gao et al. 2016) were recently used to associate regulatory regions and their target genes. These methods vary tremendously in the definition of enhancer regions, prioritization of data sources, modelling approaches, selection of validation or benchmark datasets, and the final enhancer-promoter predictions. We set off to develop a systematic, integrative and accessible approach to associate regulatory regions with their target genes while overcoming inherited problems of previous methods. We combined multiple definitions of enhancers, proposed by aforementioned publications and our own definition, to defined 286,723 enhancer regions in the human genome. We developed reg2gene $\mathrm{R}$ package, which we used to model gene expression and enhancer activity. Across-celltype signals from multiple Roadmap datasets were modelled using five different algorithms, and hierarchically majority voted. We obtained final reg2gene predictions by majority voting reg2gene models and predictions from the aforementioned publications.

We predicted 89,820 "elite" EP associations (confirmed by minimum 3 publications), whereas 435,295 EP associations were confirmed by at least 2 publications. To assess the performance of reg2gene approach, we created a set of positive and negative enhancer-gene associations combining information from experiments (Gasperini et al. 2019) and the Roadmap dataset. reg2gen achieved better statistics (accuracy, PPV, specificity) than the previously published methods.

We annotated GWAS Catalog using reg2gene predictions: $84 \%$ of SNPs was annotated to the nearest gene $(\mathrm{N}=$ 88,016 ), and $13 \%$ to the reg2gene enhancers. We further performed disease-orientated downstream analysis.

I. Patarcic: None. V. Franke: None. A. Akalin: None.

\section{C25.5}

A GWAS on data-driven 3D facial phenotypes selected by matching siblings reveals 310 genetic loci

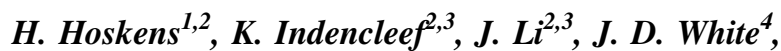
A. Ortega-Castrillon ${ }^{2,3}, J$. Wysocka ${ }^{5,6}, S$. Walsh ${ }^{7}$, S. Richmond ${ }^{8}$, J. R. Shaffer ${ }^{9,10}$, S. M. Weinberg ${ }^{9,10}$, M. D. Shriver ${ }^{4}$, H. Peeters ${ }^{1}$, P. Claes ${ }^{2,3,11}$

${ }^{I}$ Department of Human Genetics, KU Leuven, Leuven, Belgium, ${ }^{2}$ Medical Imaging Research Center, University Hospitals Leuven, Leuven, Belgium, ${ }^{3}$ Department of Electrical Engineering, ESAT/PSI, KU Leuven, Leuven,
Belgium, ${ }^{4}$ Department of Anthropology, The Pennsylvania State University, University Park, PA, United States, ${ }^{5}$ Department of Chemical and Systems Biology, Stanford University School of Medicine, Stanford, CA, United States, ${ }^{6}$ Department of Developmental Biology, Stanford University School of Medicine, Stanford, CA, United States, ${ }^{7}$ Department of Biology, Indiana University Purdue University Indianapolis (IUPUI), Indianapolis, IN, United States, ${ }^{8}$ Applied Clinical Research and Public Health, School of Dentistry, College of Biomedical and Life Sciences, Cardiff University, Cardiff, United Kingdom, ${ }^{9}$ Department of Oral Biology, Center for Craniofacial and Dental Genetics, School of Dental Medicine, University of Pittsburgh, Pittsburgh, PA, United States, ${ }^{10}$ Department of Human Genetics, Graduate School of Public Health, University of Pittsburgh, Pittsburgh, PA, United States, ${ }^{11}$ Murdoch Childrens Research Institute, Melbourne, Australia

Introduction: The role of inheritance in craniofacial morphology has repeatedly been demonstrated through twin and family studies. We further expand upon this concept by investigating and selecting facial resemblances between full siblings in order to identify genomic regions that underlie normal-range facial variation.

Methods: 3D facial shape was subdivided into distinct segments and a sibling matching experiment was performed. This led to the identification of phenotypically similar sib pairs from which their common facial traits were derived, each representing global or local aspects of facial morphology that were informative for accurately matching the siblings. Subsequently, these traits $(\mathrm{N}=1592)$ were projected onto two independent European samples $(\mathrm{N} 1=$ $4680, \mathrm{~N} 2=3566$ ) and genome-wide meta-analyses were conducted.

Results: Consistent with the literature on facial heritability, the sibling-derived traits mainly captured variations involving the full face in addition to the nasal and orbital regions. We identified 11,661 single nucleotide polymorphisms (SNPs) at 310 separate loci that reached genome-wide significance $\left(\mathrm{P}<5 \times 10^{-8}\right)$, influencing both global aspects of the face as well as local (e.g. the tip of the nose) aspects only. The majority of the associated segments represented variations in the nose.

Conclusion: We here report a data-driven approach to facial phenotyping starting from facial similarities observed between siblings. Complex shape transformations were converted into simple univariate descriptions of facial shape through vector projections, allowing for the use of standard meta-analysis techniques in testing for genotype-phenotype associations. Significant associations were found among 310 loci, further enhancing our understanding of the genetic basis of human facial shape. 
H. Hoskens: None. K. Indencleef: None. J. Li: None. J. D. White: None. A. Ortega-Castrillon: None. J. Wysocka: None. S. Walsh: None. S. Richmond: None. J. R. Shaffer: None. S.M. Weinberg: None. M.D. Shriver: None. H. Peeters: None. P. Claes: None.

\section{C25.6}

GestaltMatcher: Identifying the second patient of its kind in the phenotype space

T. Hsieh ${ }^{1}$, A. Bar-Haim ${ }^{2}$, D. Đukic ${ }^{1}$, T. J. Pantel ${ }^{3}$, M. Mensah ${ }^{3}$, Y. Gurovich ${ }^{2}$, N. Fleischer ${ }^{2}$, Y. Hanani', T. Kamphans ${ }^{4}$, G. Nadav ${ }^{2}$, P. Krawitz ${ }^{1}$

${ }^{1}$ Institute for Genomic Statistics and Bioinformatics, Bonn, Germany, ${ }^{2}$ FDNA, Boston, MA, United States, ${ }^{3}$ Charité Universitätsmedizin Berlin, Berlin, Germany, ${ }^{4}$ GeneTalk, Bonn, Germany

Introduction: Recent advances in next-generation phenotyping (NGP) tools for syndromology such as DeepGestalt have learned phenotype representations of multiple disorders by training on thousands of patient photos. However, many of Mendelian syndromes are still not represented by existing NGP tools as their disease-causing gene has not been identified yet. We therefore propose a facial gestalt matching approach to quantify the similarity among patients and further explore the unknown genotype-phenotype relationship.

Materials and Methods: We compiled a dataset consisting of 679 patients with 105 different monogenic disorders. For each individual a frontal photo and the diseasecausing mutation were available. We used additional network architectures such as FaceNet to extract facial embeddings and constructed a Clinical Face Phenotype Space (CFPS) that was corrected for the confounding effects of age and ethnicity.

Results: Given only the phenotypes as input we could visualize molecular interactions of genes by their close proximity in the CFPS. A prominent cluster was formed e.g. by BRAF, PTPNII and other genes of the MAPKinase pathway that result in disorders such as Noonan or LEOPARD syndrome and which are considered similar. Beyond this proof of concept, we were able to match two unrelated patients with a novel progeria-like facial phenotype with unknown molecular cause. They were also in close proximity to cases with mutations in LMNA and PYCRI and exome sequencing identified in these patients the same de novo mutation in a novel disease gene that can also be assigned to nuclear envelopathies.

T. Hsieh: None. A. Bar-Haim: None. D. Đukić: None. T.J. Pantel: None. M. Mensah: None. Y. Gurovich: A. Employment (full or part-time); Significant; FDNA. N.
Fleischer: A. Employment (full or part-time); Significant; FDNA. Y. Hanani: A. Employment (full or part-time); Significant; FDNA. T. Kamphans: A. Employment (full or part-time); Modest; GeneTalk. G. Nadav: A. Employment (full or part-time); Significant; FDNA. P. Krawitz: F. Consultant/Advisory Board; Modest; FDNA.

\section{C26 Mitochondrial disorder}

\section{C26.1}

Mutations in LIG3 are a novel cause of mitochondrial neurogastrointestinal encephalomyopathy

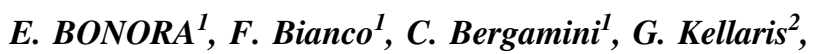
F. Ullah ${ }^{2}$, F. Isidori ${ }^{1}$, I. Liparulo ${ }^{I}$, C. Diquigiovanni, L. Masin', M. G. Cratere ${ }^{3}$, E. Boschetti, ${ }^{1}$, V. Papa ${ }^{1}$, A. Maresca ${ }^{I}$, G. Cenacchi ${ }^{1}$, R. Casadio ${ }^{1}$, P. Martelli ${ }^{I}$, I. Matera ${ }^{4}$, I. Ceccherini ${ }^{4}$, R. Fato ${ }^{1}$, G. Raiola ${ }^{5}$, S. Arrigo $^{4}$, S. Signa ${ }^{4}$, M. S. Severino ${ }^{4}$, P. Striano ${ }^{4}$, C. Fiorillo ${ }^{4}$,

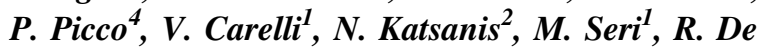
Giorgio $^{6}$

${ }^{1}$ UNIVERSITY OF BOLOGNA, BOLOGNA, Italy, ${ }^{2}$ Duke University, Durham, NC, United States, ${ }^{3}$ San Raffaele Scientific Institute, Milano, Italy, ${ }^{4}$ IRCCS G. Gaslini Institute, Genova, Italy, ${ }^{5}$ Pugliese-Ciaccio Hospital, Catanzaro, Italy, ${ }^{6}$ University of Ferrara, Ferrara, Italy

We identified the novel gene mutations in three male siblings, born from healthy unrelated parents, presenting with neurogastrointestinal encephalomyopathy featuring severe gut dysmotility (i.e. chronic intestinal pseudoobstruction, CIPO) and neurological impairment with a peculiar leukoencephalopathy characterized by a "leopardskin pattern". Whole exome sequencing (WES) performed on peripheral blood-derived DNA of the affected siblings revealed two novel heterozygous variants, p.K537N (paternal) and p.G964R (maternal) in LIG3, a DNA ligase involved in nuclear and mitochondrial DNA (mtDNA) maintenance. lig3 ablation in zebrafish reproduced brain alterations and impaired gut transit, reminiscent of the human condition. Such phenotype was rescued with wildtype LIG3 mRNA, but not with mRNA encoding the two variants, indicating a loss-of-function mechanism. The $\mathrm{p}$. $\mathrm{K} 537 \mathrm{~N}$ variant, mapping to the last base-pair before the donor splice-site, induced the skipping of the corresponding exon, in patient-derived skin fibroblasts. The p.G964R variant, residing in a C-terminal binding site for XRCC1, impaired LIG3/XRCC1 binding. Both mutations induced significant mtDNA depletion in skeletal muscle and fibroblasts resulting in mitochondrial dysfunction in cells. Compared to controls, mutant skin fibroblasts revealed 
abnormal mitochondria with disorganized, degenerated cristae, a decreased ATP synthesis, and decreased mitochondrial membrane potential. Increase in cell viability was obtained via glutamine addition to the culture medium. In conclusion, we identified a novel form of mitochondrial gastrointestinal encephalomyopathy due to mutations in LIG3, a gene not previously associated to any human disease.

This work was supported by Telethon Grant GGP15171.

E. Bonora: None. F. Bianco: None. C. Bergamini: None. G. Kellaris: None. F. Ullah: None. F. Isidori: None. I. Liparulo: None. C. Diquigiovanni: None. L. Masin: None. M.G. Cratere: None. E. Boschetti: None. V. Papa: None. A. Maresca: None. G. Cenacchi: None. R. Casadio: None. P. Martelli: None. I. Matera: None. I. Ceccherini: None. R. Fato: None. G. Raiola: None. S. Arrigo: None. S. Signa: None. M.S. Severino: None. P. Striano: None. C. Fiorillo: None. P. Picco: None. V. Carelli: None. N. Katsanis: None. M. Seri: None. R. De Giorgio: None.

\section{C26.2}

Mutations in POLRMT impair mitochondrial transcription and are associated with a spectrum of mitochondrial disease presentations

M. Oláhovál, B. Peter ${ }^{2}$, H. Diaz ${ }^{2}$, Z. Szilagyi ${ }^{2}$, E. W. Sommerville ${ }^{I}$, E. L. Blakely ${ }^{I}$, J. Collier ${ }^{1}$, V. Stránecky ${ }^{3}$, H. Hartmannová ${ }^{3}$, A. J. Bleyer, K. L. McBride ${ }^{5}$ S. A. Bowden ${ }^{5}$, Z. Korandová ${ }^{6}$,

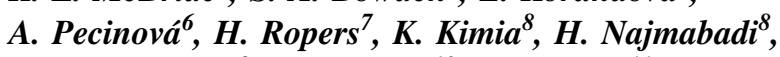
M. Tarnopolsky ${ }^{9}$, L. I. Brady ${ }^{10}$, N. Weaver ${ }^{11}$, C. E. Prada ${ }^{12}$, T. Mráček ${ }^{6}$, S. Kmoch ${ }^{3}$, G. S. Gorman', M. Falkenberg ${ }^{2}$, C. Gustafsson ${ }^{2}$, R. W. Taylor ${ }^{1}$

${ }^{1}$ Wellcome Centre for Mitochondrial Research, Newcastle University, Newcastle upon Tyne, United Kingdom, ${ }^{2}$ Department of Medical Biochemistry and Cell Biology, University of Gothenburg, Gothenburg, Sweden, ${ }^{3}$ Research Unit for Rare Diseases, Charles University, Prague, Czech Republic, ${ }^{4}$ Section on Nephrology, Wake Forest School of Medicine, Winston-Salem, NC, United States, ${ }^{5}$ Nationwide Children's Hospital, The Ohio State University College of Medicine, Columbus, OH, United States, ${ }^{6}$ Department of Bioenergetics, Institute of Physiology of the Czech Academy of Sciences, Prague, Czech Republic, ${ }^{7}$ Max Planck Institute for Molecular Genetics, Berlin, Germany, ${ }^{8}$ Genetics Research Center, University of Social Welfare and Rehabilitation Sciences, Tehran, Iran, Islamic Republic of, ${ }^{9}$ Division of Neuromuscular and Neurometabolic Diseases, McMaster University, Hamilton, ON, Canada, ${ }^{10}$ Department of Pediatrics, McMaster University Children's Hospital, Hamilton, ON, Canada, ${ }^{11}$ Division of Human
Genetics, Cincinnati Children's Hospital Medical Center, Cincinatti, OH, United States, ${ }^{12}$ Division of Human Genetics, Cincinnati Children's Hospital Medical Center, Cincinnati, OH, United States

Introduction: The majority of mitochondrial disorders result from mutations in components of the nuclear-encoded mitochondrial DNA (mtDNA) maintenance machinery and oxidative phosphorylation (OXPHOS) subunits; the role of the mtDNA transcription machinery remains relatively unknown. The mitochondrial RNA polymerase (POLRMT) is the sole RNA polymerase in mitochondria and is responsible for mtDNA transcription.

Patients and Methods: Using whole-exome sequencing, we identified novel recessive and dominant POLRMT variants in five individuals presenting with a variety of clinical problems, ranging from global developmental delay, hypotonia and growth defects in childhood to late onset progressive external ophthalmoplegia (PEO). Where investigated, these defects were accompanied by either a mosaic cytochrome $c$ oxidase deficiency in skeletal muscle and/or multiple respiratory chain enzyme deficiencies. Mitochondrial mRNA and OXPHOS protein levels were assessed in mutant POLRMT fibroblasts. In addition, we studied recombinant mutant POLRMT proteins to determine the effect of POLRMT variants on mitochondrial transcriptional activity in vitro.

Results: Functional characterisation of patient fibroblasts revealed a defect in mitochondrial mRNA synthesis, although no mtDNA deletions or copy number abnormalities were identified. Mild decreases in the levels of both OXPHOS subunits and fully-assembled complexes were observed in vivo, whilst functional in vitro characterisation of the investigated recombinant POLRMT variants revealed that patient mutations exhibited variable, but deleterious effects on mitochondrial transcription.

Conclusion: Our results demonstrate that pathogenic variants in the POLRMT gene can cause a spectrum of clinical phenotypes ranging from childhood-onset developmental delay to late-onset PEO and emphasise the importance of defective mitochondrial transcription as a disease mechanism.

M. Oláhová: None. B. Peter: None. H. Diaz: None. Z. Szilagyi: None. E.W. Sommerville: None. E.L. Blakely: None. J. Collier: None. V. Stránecký: None. H. Hartmannová: None. A.J. Bleyer: None. K.L. McBride: None. S.A. Bowden: None. Z. Korandová: None. A. Pecinová: None. H. Ropers: None. K. Kimia: None. H. Najmabadi: None. M. Tarnopolsky: None. L.I. Brady: None. N. Weaver: None. C.E. Prada: None. T. Mráček: None. S. Kmoch: None. G.S. Gorman: None. M. Falkenberg: None. C. Gustafsson: None. R.W. Taylor: None. 


\section{C26.3}

Mutations in the MRPS28 gene encoding the small mitoribosomal subunit protein $\mathrm{bS} 1 \mathrm{~m}$ in a patient with intrauterine growth retardation, craniofacial dysmorphism and multisystemic involvement

\section{J. Pulman', B. Ruzzenente ${ }^{1}$, L. Bianchi ${ }^{I}$, M. Rio ${ }^{2}$,} N. Boddaert ${ }^{3}$, A. Munnich ${ }^{4}$ A. Rötig ${ }^{1}$, M. Metodiev ${ }^{1}$

${ }^{1}$ UMR1163, Université Paris Descartes, Sorbonne Paris Cité, Institut IMAGINE, Paris, France, ${ }^{2}$ Departments of Pediatrics, Neurology and Genetics, Hôpital NeckerEnfants-Malades, Paris, France, ${ }^{3}$ Department of pediatric radiology, INSERM 1000 and INSERM UMR1163, Hôpital Necker-Enfants-Malades AP-HP, Université Paris Descartes - Sorbonne Paris Cité, Institut Imagine, Paris, France, ${ }^{4}$ INSERM UMR1163, Université Paris Descartes Sorbonne Paris Cité, Institut Imagine, Paris, France Departments of Pediatrics, Neurology and Genetics, Hôpital Necker-Enfants-Malades, Paris, France

Mitochondria contain a dedicated translation system, which is responsible for the intramitochondrial synthesis of $13 \mathrm{mtDNA}$-encoded polypeptides essential for the biogenesis of oxidative phosphorylation (OXPHOS) complexes I and III to V. Mutations in nuclear genes encoding factors involved in mitochondrial translation result in isolated or multiple OXPHOS deficiencies and mitochondrial disease. Here, we report the identification of disease-causing variants in the MRPS28 gene, encoding the small mitoribosomal subunit ( $\mathrm{mtSSU}$ ) protein $\mathrm{bS} 1 \mathrm{~m}$ in a patient with intrauterine growth retardation, craniofacial dysmorphism and developmental delay. Whole exome sequencing helped identify a seemingly homozygous missense variant NM_014018.2:c.356A $>$ G, p. (Lys119Arg) which affected a highly conserved lysine residue. The variant was present in the mother in a heterozygous state, but not in the father who likely carried a large deletion spanning exon 2 and parts of introns 1 and 2 that could account for the apparent homozygosity of the patient. PCR amplification and Sanger sequencing of MRPS28 cDNA from patient fibroblasts revealed the presence of a truncated MRPS28 transcript, which lacked exon 2. Molecular and biochemical characterization of patient fibroblasts revealed a decrease in the abundance of the $\mathrm{bS} 1 \mathrm{~m}$ protein, decreased abundance of assembled $\mathrm{mtSSU}$ and inhibited mitochondrial translation. Consequently, OXPHOS biogenesis and cellular respiration were compromised in these cells. Expression of wild-type MRPS28 restored mitoribosomal assembly, mitochondrial translation and OXPHOS biogenesis, thereby demonstrating the deleterious nature of the identified MRPS 28 variants. Thus, MRPS28 joins the increasing number of nuclear genes encoding mitoribosomal structural proteins linked to mitochondrial disease.

J. Pulman: None. B. Ruzzenente: None. L. Bianchi: None. M. Rio: None. N. Boddaert: None. A. Munnich: None. A. Rötig: None. M. Metodiev: None.

\section{C26.4}

Brain-on-a-chip - a neurophysiological model of MELAS disease and comorbid psychopathology

\section{T. Kozicz ${ }^{1}$, T. Klein-Gunnewiek ${ }^{2}$, D. Cassiman ${ }^{3}$, T. Nelson ${ }^{1}$, R. Rodenburg ${ }^{2}$, E. Perales-Clemente ${ }^{1}$, E. Morava ${ }^{1}$, N. Nadif Kasri ${ }^{2}$}

${ }^{1}$ Mayo Clinic, Rochester, MN, United States, ${ }^{2}$ Radboudumc, Nijmegen, Netherlands, ${ }^{3}$ UZ Leuven, Leuven, Belgium

Mitochondrial dysfunction is increasingly recognized in the ethology of psychiatric disorders. More than half of individuals with mitochondrial encephalomyopathy, lactic acidosis and stroke-like episodes (MELAS), who carry the pathogenic variant $m .3243 \mathrm{~A}>\mathrm{G}$ in their mtDNA, have depression as a comorbidity. Mechanistic insights into mitochondrial dysfunction in psychiatric diseases are largely elusive. Here, we reprogramed skin fibroblasts from MELAS patients with comorbid depression into induced pluripotent stem cells (iPSCs). These cells exhibited isogenic nuclear DNA but different levels of mtDNA heteroplasmy (i.e.: 0 and $>80 \%$ ) in $\mathrm{m} .3243 \mathrm{~A}>\mathrm{G}$ mutation. Next, we differentiated iPS cells into excitatory cortical neurons (iNeurons). We found that high levels of m.3243A $>$ G heteroplasmy ( $>80 \%$ ) resulted in mitochondrial dysfunction, accompanied by reduced span and complexity of the dendritic arbors, as well as a reduction in axonal mitochondria. Furthermore, we found fewer excitatory synapses and accordingly a lower frequency of spontaneous postsynaptic currents. Neuronal network recordings from microelectrode arrays showed less activity in general, as well as a decrease in synchronous network bursts. We now provide conceptual advance on how impaired neuron energy metabolism disrupts synaptic structure and function, dendritic complexity, as well as network activity and synchronicity. These specific neuronal features are impaired to various degrees in psychiatric diseases as well. Overall, our results show that iNeurons are an effective disease model for improving our understanding of the role of mitochondrial dysfunction in the aetiology of psychopathology. iNeurons could also serve as screening platforms for novel genetic and/or pharmacological therapeutic discoveries. Support: Tjallingh Roorda Foundation, Stichting Stofwisselkracht, Marriott Family

T. Kozicz: None. T. Klein-Gunnewiek: None. D. Cassiman: None. T. Nelson: None. R. Rodenburg: None. E. 
Perales-Clemente: None. E. Morava: None. N. Nadif Kasri: None.

\section{C26.5}

The homozygous variant c.797G $>A / p .(C y s 266 T y r)$ in $P I S D$ is associated with a spondyloepimetaphyseal dysplasia with large epiphyses and disturbed mitochondrial function

L. von Elsner ${ }^{1}$, K. M. Girisha ${ }^{2}$, K. Neethukrishna ${ }^{2}$, M. Muranjan ${ }^{3,4}$, A. Shukla', G. S. Bhavani', G. Nishimura ${ }^{5}$, K. Kutsche ${ }^{1}$, G. Mortier ${ }^{6}$

${ }^{1}$ Institute of Human Genetics, University Medical Center Hamburg-Eppendorf, Hamburg, Germany, ${ }^{2}$ Department of Medical Genetics, Kasturba Medical College, Manipal Academy of Higher Education, Manipal, India, ${ }^{3}$ Department of Clinical Genetics, Seth GS Medical College and KEM Hospital, Mumbai, India, ${ }^{4}$ Consultant in Clinical Genetics, P.D. Hinduja National Hospital \& MRC, Mumbai, India, ${ }^{5}$ Department of Pediatric Imaging, Tokyo Metropolitan Children's Medical Center, Fuchu, Japan, ${ }^{6}$ Centre of Medical Genetics, University of Antwerp \& University Hospital Antwerp, Antwerp, Belgium

Spondyloepimetaphyseal dysplasias (SEMD) are a group of genetically heterogeneous skeletal disorders characterized by abnormal vertebral bodies and epimetaphyseal abnormalities. We investigated two families with a new SEMD type with one proband each. They showed mild facial dysmorphism, flat vertebral bodies (platyspondyly), large epiphyses, metaphyseal dysplasia, and hallux valgus as common clinical features. By trio exome sequencing, the homozygous missense variant c.797G $>$ A p.(Cys266Tyr) in PISD was found in both affected individuals. PISD encodes phosphatidylserine decarboxylase that is localized in the inner mitochondrial membrane and catalyzes the decarboxylation of phosphatidylserine to phosphatidylethanolamine (PE) in mammalian cells. PE occurs at high abundance in the mitochondrial membranes, and structural properties of this phospholipid play an important role for various mitochondrial functions. To characterize the functional effects of the PISD missense variant we used fibroblasts derived from one patient and healthy individuals. Western blot analysis revealed no difference in PISD protein amount in patient- and control-derived fibroblasts. In contrast to the control fibroblasts, patient cells showed fragmented mitochondrial morphology. Treatment of patient fibroblasts with MG-132 or staurosporine to induce oxidative stress followed by the activation of the intrinsic apoptosis pathway showed significantly decreased cell viability with increased caspase- 3 and -7 activation. Remarkably, ethanolamine supplementation largely restored cell viability and enhanced apoptosis in MG-132-stressed patient cells. Our data demonstrate that the bi-allelic hypomorphic PISD variant p.(Cys266Tyr) is associated with a novel SEMD form, which may be treatable with PE or ethanolamine administration.

L. von Elsner: None. K.M. Girisha: None. K. Neethukrishna: None. M. Muranjan: None. A. Shukla: None. G.S. Bhavani: None. G. Nishimura: None. K. Kutsche: None. G. Mortier: None.

C26.6

SSBP1 mutations cause a complex optic atrophy spectrum disorder with mitochondrial DNA depletion

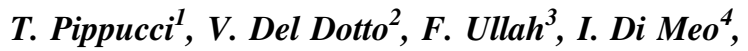
P. Magini, M. Gusic ${ }^{5}$, A. Maresca $^{6}$, L. Caporali $^{6}$, F. Palombo ${ }^{6}$, F. Tagliavini ${ }^{6}$, E. H. Baugh ${ }^{7}, C$. La

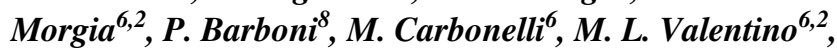

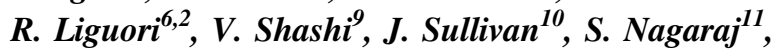
E. Bertini ${ }^{12}$, R. Carrozzo $^{12}$, F. Emma ${ }^{12}$, I. Cutcutache ${ }^{13}$, M. Armstrong ${ }^{13}$, M. Page ${ }^{13}$, N. Stong ${ }^{7}$, E. Davies $^{3}$, D. Karall ${ }^{14}$, S. Boesch ${ }^{14}$, M. Seri ${ }^{1,15}$, M. Falkenberg ${ }^{16}$,

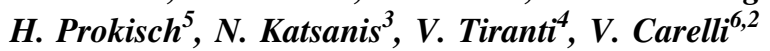

${ }^{I}$ Medical Genetics Unit, Sant'Orsola-Malpighi University Hospital, Bologna, Italy, ${ }^{2}$ Unit of Neurology, Department of Biomedical and NeuroMotor Sciences (DIBINEM), University of Bologna, Bologna, Italy, ${ }^{3}$ Center for Human Disease Modeling, Duke University, Durham, NC, United States, ${ }^{4}$ Molecular Neurogenetics Unit, IRCCS Foundation, C. Besta Neurological Institute, Milano, Italy, ${ }^{5}$ Institute of Human Genetics, Helmholtz Zentrum, Munchen, Germany, ${ }^{6}$ IRCCS Institute of Neurological Sciences of Bologna, Bellaria Hospital, Bologna, Italy, ${ }^{7}$ Institute for Genomic Medicine, Columbia University Medical Center, New York, $N Y$, United States, ${ }^{8}$ Scientific Institute San Raffaele, MIlano, Italy, ${ }^{9}$ Division of Medical Genetics, Department of Pediatrics, Duke University Medical Center, Durham, NC, United States, ${ }^{10}$ Division of Medical Genetics, Department of Pediatrics, Duke University Medical Center, Bologna, Italy, ${ }^{11}$ Division of Nephrology, Department of Pediatrics, Duke University Medical Center, Durham, NC, United States, ${ }^{12}$ Division of Nephrology, Department of Pediatric Subspecialties, Bambino Gesù Children's Hospital IRCCS, Roma, Italy, ${ }^{13}$ UCB Celltech, Slough, United Kingdom, ${ }^{14}$ Department of Neurology, Medical University, Innsbruck, Austria, ${ }^{15}$ Department of Medical and Surgical Sciences (DIMEC), University of Bologna, Bologna, Italy, Bologna, Italy, ${ }^{16}$ Department of Medical Biochemistry and Cell Biology, University of Gothenburg, Gothenburg, Sweden 
Introduction: Single strand binding protein 1 (SSBP1) is a key tetrameric protein for replication of mitochondrial DNA (mtDNA), which together with the replicative polymerase POLG and the helicase Twinkle constitutes the "minimal" replisome. Pathogenic mutations in POLG/Twinkle impair mtDNA replication and cause mitochondrial dysfunction leading to human infantile or late-onset phenotypes.

Materials and Methods: We analyzed by whole exome sequencing five families with an optic atrophy spectrum disorder, including retinal dystrophy and kidney insufficiency, associated with mtDNA depletion. Functional experiments evaluating effects of potentially pathogenic variants on protein amount and multimerization, mtDNA replication and cellular bioenergetics were performed in vitro and/or in patients' fibroblasts. Optic nerve size was measured in zebrafish after knock-down and wildtype or mutant overexpression.

Results: We identified four heterozygous and one homozygous missense mutations in the SSBP1 gene. They affect mtDNA maintenance and replication, leading to mtDNA depletion, which resulted in a variable phenotype of impaired oxidative phosphorylation, observed either in fibroblasts or in affected tissues (kidney and skeletal muscle). SSBP1 suppression in zebrafish reduced optic nerve size, a phenotype rescued by wild-type protein but not by the SSBP1 mutations, suggesting that all dominant mutations induced loss of function, whereas the recessive behaved as a hypomorph.

Conclusion: Mutations in SSBP1 cause defects in mtDNA replication, mitochondrial dysfunction and human disease. Further investigations will contribute to improve knowledge about mtDNA replication, and enlarge the landscape of phenotypic expression of mitochondrial diseases in humans.

Funding: Italian Ministry of Health (Ricerca Corrente) to $\mathrm{VC}$

T. Pippucci: None. V. Del Dotto: None. F. Ullah: None. I. Di Meo: None. P. Magini: None. M. Gusic: None. A. Maresca: None. L. Caporali: None. F. Palombo: None. F. Tagliavini: None. E.H. Baugh: None. C. La Morgia: None. P. Barboni: None. M. Carbonelli: None. M.L. Valentino: None. R. Liguori: None. V. Shashi: None. J. Sullivan: None. S. Nagaraj: None. E. Bertini: None. R. Carrozzo: None. F. Emma: None. I. Cutcutache: None. M. Armstrong: None. M. Page: None. N. Stong: None. E. Davies: None. D. Karall: None. S. Boesch: None. M. Seri: None. M. Falkenberg: None. H. Prokisch: None. N. Katsanis: None. V. Tiranti: None. V. Carelli: None.

\section{C27 Developmental disorders 2}

\section{C27.1}

Diagnostic utility of genome-wide DNA methylation testing in genetically unsolved patients with suspected hereditary conditions

\section{B. sadikovic}

London Health Sciences Centre, Canada, London, ON, Canada

Conventional genetic testing of patients with neurodevelopmental presentations and congenital anomalies (ND/CA), i.e., the analysis of sequence and copy number variants, leaves a substantial proportion of the patients unexplained. Some of these cases have been shown to result from DNA methylation defects at a single locus (epi-variants), while others can exhibit syndrome-specific DNA methylation changes across multiple loci (epi-signatures). Here, we investigate the clinical diagnostic utility of genome-wide DNA methylation analysis of peripheral blood in unresolved ND/CA patients. We generate a computational model enabling concurrent detection of 14 syndromes using peripheral blood DNA methylation data with full accuracy. We demonstrate the ability of this model to resolve 67 patients with uncertain clinical diagnoses, some of whom had VUS's in the related genes. We show that the provisional diagnoses can be ruled out in many of the cases, some of whom are shown by our model to have other diseases initially not considered. By applying this model to a cohort of $965 \mathrm{ND} / \mathrm{CA}$ patients without a previous diagnostic assumption and separate assessment of rare epi-variants in this cohort, we identify 15 patients with syndromic genetic disorders, 12 patients with imprinting and trinucleotide repeat expansion disorders, as well as 106 patients with rare epi-variants, a portion of which involved genes clinically or functionally linked to the patients' phenotype. This study demonstrates that genomic DNA methylation analysis can facilitate the molecular diagnosis of unresolved clinical cases, and highlights the potential value of epigenomic testing in the routine clinical assessment of patients with ND/CA.

B. sadikovic: None.

\section{C27.2}

Multiomics Approach to Diagnosing Undiagnosed Patients

M. T. Wheeler, J. N. Kohler, D. E. Bonner, D. B. Zastrow, C. Reuter, M. Majcherska, L. Fernandez, C. McCormack, S. Marwaha, C. Curnin, A. Dries, M. Ruzhnikov, J. Hom, J. Sampson, P. G. Fisher, Undiagnosed Diseases Network, E. A. Ashley, J. A. Bernstein 
Stanford University School of Medicine, Palo Alto, CA, United States

Introduction: Patients with undiagnosed syndromes represent the most challenging and rare manifestations of disease. The Center for Undiagnosed Diseases at Stanford (CUD), a clinical site of the NIH funded Undiagnosed Diseases Network, evaluates patients with rare, undiagnosed diseases in an effort to diagnose patients, improve mechanistic knowledge of diseases, and accelerate bioinformatics tool development.

Methods: We reviewed the first 400 applicants to the CUD to identify phenotypic characteristics, demographics, and prior genetics evaluations. 147 patients were enrolled with both subjective and objective evidence of undiagnosed disease. Exome sequencing, sequence reanalysis focused on novel variant and gene discovery, and beyond exome approaches including short- and long-read genome sequencing, RNAseq, untargeted metabolomics, immunomics, and model systems were applied to attempt to find a diagnosis for each of these participants.

Results: The most common predominantly affected systems of applicants include neurologic, musculoskeletal, and immunologic. Among the 147 enrolled participants to date, 114 have had evaluations completed, 34 participants received a confirmed diagnosis using a variety of clinical, genomic and research approaches. Specific evaluations and additional research studies are determined on a case-by-case bases by CUD clinician-scientists due to the diversity of presentation and prior work-up of each participant. To date, the CUD has found a strong candidate diagnosis for 10 (9\%) patients. Each candidate diagnosis is undergoing further functional investigation.

Conclusions: Together with local and international collaborators, a multiomic approach to undiagnosed patients has found success in the diagnosis and characterization of rare diseases. Supported by NIH Common Fund U01HG010218

M.T. Wheeler: None. J.N. Kohler: None. D.E. Bonner: None. D.B. Zastrow: None. C. Reuter: None. M. Majcherska: None. L. Fernandez: None. C. McCormack: None. S. Marwaha: None. C. Curnin: None. A. Dries: None. M. Ruzhnikov: None. J. Hom: None. J. Sampson: None. P.G. Fisher: None. E.A. Ashley: E. Ownership Interest (stock, stock options, patent or other intellectual property); Modest; Personalis Inc, Deep Cell Inc. J.A. Bernstein: None.

\section{C27.3}

Mutated epigenetic modifiers in CYLD cutaneous syndrome
H. R. Davies ${ }^{1}$, K. Hodgson ${ }^{2}$, E. Schwalbe ${ }^{3}$, J. Coxhead ${ }^{2}$, N. Sinclair ${ }^{2}$, X. Zou ${ }^{4}$, S. Cockell ${ }^{2}$, A. Husain ${ }^{5}$, S. NikZainal $^{6}$, N. Rajan ${ }^{2}$

${ }^{I}$ Wellcome Trust Sanger Institute, Hinxton, United Kingdom, ${ }^{2}$ Institute of Genetic Medicine, Newcastle upon Tyne, United Kingdom, ${ }^{3}$ Department of Applied Sciences, Northumbria University, Newcastle upon Tyne, United Kingdom, ${ }^{4}$ MRC Cancer Unit, Cambridge, United Kingdom, ${ }^{5}$ Department of Pathology, Royal Victoria Infirmary, Newcastle upon Tyne, United Kingdom, ${ }^{6}$ Academic Department of Medical Genetics, Cambridge, United Kingdom

Introduction: Patients with rare germline mutations in the tumour suppressor $C Y L D$ progressively develop multiple skin tumours over their lifetime. The diverse histological patterns of hair follicle tumours seen in this syndrome suggest genetic heterogeneity across these tumours.

Methods: To investigate this, we comprehensively profiled the genomic landscape of 42 benign and malignant tumours across 13 individuals in 4 multigenerational families with CYLD cutaneous syndrome (CCS; syn. Brooke-Spiegler syndrome).

Results: We found recurrent mutations in epigenetic modifiers DNMT3A and BCOR in $29 \%$ of tumours. Integrated genomic and methylation profiling suggest that mutant DNMT3A drives tumourigenesis mechanistically through $\mathrm{Wnt} / \mathrm{B}$-catenin pathway signaling. In malignant tumours, epigenetic modifiers CREBBP, KDM6A and $E P 300$ were mutated. Some patients were heterozygous for loss of function mutations in $M B D 4$, with biallelic mutations demonstrated in a poorly differentiated malignant skin tumour with an associated increase in mutational burden and the accrual of epigenetic modifiers. To obtain insights into mechanisms that cause these mutations, mutational signature analysis was performed, and revealed UV-related signatures in sun-exposed lesions but not sun-protected tumours suggesting that non-UV-related mechanisms contribute towards CCS tumourigenesis. We also demonstrate that cutaneous tumours that metastasize to the lung carry a targetable recurrent driver mutation in $A K T$, and a $\mathrm{UV}$ signature, supporting the origin from the skin.

Conclusion: These findings add new dimensions to the existing paradigms of UV-induced skin cancer and highlight the utility of studying rare disease to gain novel insights into genetic mechanisms of tumour formation. NR was funded by a Wellcome Trust Fellowship WT097163MA.

H.R. Davies: None. K. Hodgson: None. E. Schwalbe: None. J. Coxhead: None. N. Sinclair: None. X. Zou: None. S. Cockell: None. A. Husain: None. S. Nik-Zainal: None. N. Rajan: None. 


\section{C27.4}

Identification and characterization of NEPRO-related skeletal dysplasia resembling cartilage hair hypoplasia

\section{Narayanan ${ }^{I}$, N. Kausthubham ${ }^{I}$, G. S. Bhavani ${ }^{1}$, H. Shah ${ }^{1}$, A. Shukla ${ }^{1}$, G. Mortier ${ }^{2}$, K. M. Girisha ${ }^{1}$}

${ }^{1}$ Kasturba Medical College, Manipal, Manipal Academy of Higher Education, Manipal, Manipal, India, ${ }^{2}$ Center of Medical Genetics, University of Antwerp and Antwerp University Hospital,, Antwerp, Belgium, Belgium

Cartilage-hair hypoplasia (CHH), anauxetic dysplasia 1 and anauxetic dysplasia 2 are rare metaphyseal dysplasias caused by biallelic pathogenic variants in $R M R P$ and $P O P 1$, which encode the components of RNAse-MRP endoribonuclease complex (RMRP) in ribosomal biogenesis pathway. Nucleolus and neural progenitor protein (NEPRO), encoded by NEPRO (C3ORF17) interacts with multiple protein subunits of RMRP. We report a biallelic pathogenic variant in NEPRO, which could disrupt the normal function of RMRP and cause a similar skeletal dysplasia. We ascertained a 6-year-old girl, with skeletal dysplasia resembling $\mathrm{CHH}$. Analysis of parents-child trio exomes and effect of the variant on protein structure and stability were performed. Two families with four affected individuals with a skeletal dysplasia and a homozygous missense variant, c. 280C $>\mathrm{T}$ (p. Arg94Cys) in NEPRO, were identified from literature and their phenotype was critically re-evaluated. $R M R P$ and $P O P 1$ in proband did not harbor any causative variant. Trio exome sequencing identified a possible homozygous candidate variant, c. $435 \mathrm{G}>\mathrm{C}$ (p. Leu145Phe) in NEPRO. All the five affected individuals have short stature, acromelia, skin laxity, joint hypermobility and dislocations. They also have short metacarpals, broad middle phalanges, narrow acetabular angle and metaphyseal irregularities. Protein modeling and stability prediction showed that the mutant protein has decreased stability. The reported variants are in the same domain of the protein. We report the identification and characterization of a new syndrome caused by biallelic variants in NEPRO in five patients with features resembling $\mathrm{CHH}$ and thus expand the phenotypic and genotypic spectrum of ribosome biogenesis pathway related skeletal dysplasia.SB/SO/HS/ $005 / 2014$

D. Narayanan: None. N. Kausthubham: None. G.S. Bhavani: None. H. Shah: None. A. Shukla: None. G. Mortier: None. K.M. Girisha: None.

\section{C27.5}

Impact of $A L P K 1$ causative variant in ROSAH syndrome, a newly characterised retinal and multisystem autosomal dominant disorder
R. V. Jamieson ${ }^{1,2,3}$, A. Sabri ${ }^{1}$, J. R. Grigg ${ }^{4}$, A. Cheng ${ }^{\text {I, }}$ T. H. Loi ${ }^{I}$

${ }^{I}$ Eye Genetics Research Unit, Children's Medical Research Institute, The Children's Hospital at Westmead, Save Sight Institute, University of Sydney, Sydney, Australia, ${ }^{2}$ Discipline of Genomic Medicine, Children's Hospital at Westmead Clinical School, Faculty of Medicine and Health, University of Sydney, Sydney, Australia, ${ }^{3}$ Department of Clinical Genetics, Western Sydney Genetics Program, The Children's Hospital at Westmead, Sydney Children's Hospitals Network, Sydney, Australia, ${ }^{4}$ Save Sight Institute, Faculty of Medicine and Health, University of Sydney, Sydney, Australia

Introduction: ROSAH syndrome: Retinal dystrophy, Optic nerve edema, Splenomegaly, Anhidrosis and Headaches is newly described. Independent genome and exome sequencing, identified the same causative variant in ALPKI in five families*. We present phenotypic and functional studies characterising additional inflammatory, ciliary and centrosomal abnormalities in six affected individuals from one of the families.

Materials and Methods: ROSAH syndrome patients heterozygous for $A L P K 1, \quad$ c.710C $>\mathrm{T}$, [p.Thr237Met]), underwent immunological, cerebral MRI, bone marrow and ophthalmic investigations. Centrosomal and primary ciliary analyses were conducted in human fibroblasts, ARPE-19 and HeLa cells. HeLa cells were subject to overexpression of mutant and wildtype ALPK1, and ALPK1 knockdown. ALPK1 isoform characterisation was undertaken in human and mouse tissues.

Results: Additional clinical features indicated innate immunity dysfunction with marked susceptibility to viral infections, and indication of abnormal inflammatory responses including cerebral manifestations. Primary ciliary and centrosomal abnormalities in cell culture investigations suggested a possible gain of function disease mechanism. ALPK1 isoform differences were identified in human and mouse retina providing additional insight to disease mechanisms.

Conclusion: Heterozygous abnormality in ALPKI leads to the multisystem ROSAH disorder through impact on retinal and other tissues. There are primary cilia and centrosomal abnormalities as well as indicators of immune system dysfunction, and a likely gain of function disease mechanism.

*ALPK1 missense pathogenic variant in five families leads to ROSAH syndrome, an ocular multisystem autosomal dominant disorder. Williams LB $^{\sim}$, Javed A $\sim$, Sabri $\mathrm{A}^{\sim}$, Morgan $\mathrm{D}^{\sim}$ et al., Jamieson $\mathrm{RV}^{\wedge}$, DeAngelis $\mathrm{MM}^{\wedge}$. Genetics in Medicine, Accepted 12 February 2019. Equal first; ^Equal last authors. 
R.V. Jamieson: None. A. Sabri: None. J.R. Grigg: None. A. Cheng: None. T.H. Loi: None.

\section{C27.6}

New mechanism for retinal degeneration on chrXq27.1

J. C. Gardner ${ }^{1}$, K. Jovanovich, D. Ottaviani, J. Jackson ${ }^{1}$, A. T. Moore ${ }^{1,2,3}$, K. Ziaka ${ }^{1}$, K. Hau ${ }^{1}$, A. Lane ${ }^{I}$, M. Michaelides ${ }^{1,2}$, M. E. Cheetham ${ }^{I}$, A. J. Hardcastle ${ }^{I}$

${ }^{1}$ UCL Institute of Ophthalmology, 11-43 Bath Street, EC1V 9EL, London, United Kingdom, ${ }^{2}$ Moorfields Eye Hospital, 162 City Road, ECIV 2PD, London, United Kingdom, ${ }^{3}$ Ophthalmology Department, University of California San Francisco School of Medicine, San Francisco, CA, United States

$\mathrm{X}$-linked retinal degenerations are a heterogeneous group of disorders that lead to progressive loss of vision and photoreceptor cell death. Following exclusion of known genes in a multi-generation family, we mapped the locus to Xq26.3-27.2. Analysis of genome sequence data excluded coding variants in all genes in the interval, and revealed split-reads, indicative of a potential structural variant. Using long range PCR and DNA walking, we identified a $57 \mathrm{~Kb}$ inter-chromosomal insertion of chr9p24.3, within an intergenic palindromic region on chrXq27.1. Structural variants can modulate mechanisms of gene regulation, affect gene dosage, or as a result of position effects, influence the expression of genes proximal or distal to the breakpoints. We hypothesised that retinal degeneration is caused by dysregulated gene expression, either near the palindrome, or driven by expression of chr9p24.3 transcripts from the $\mathrm{X}$-chromosome. To explore the mechanism of disease with the insertion in genomic and cellular context, we reprogrammed patient fibroblasts to induced pluripotent stem cells (iPSCs) and differentiated 3D retinal organoids. RNAseq analysis of patient retinal organoids, compared to controls, revealed transcriptional dysregulation at this locus. Further analysis of the downstream effects includes immunostaining to assess retinal organoid morphology and protein expression. We have subsequently identified another family with a different inter-chromosomal insertion within the same intergenic palindromic region of chrX. These studies identify a new genomic mechanism for retinal degeneration, and the utility of patient iPSC derived retinal organoids to investigate dysregulated gene expression as the likely cause of disease.

J.C. Gardner: None. K. Jovanovich: None. D. Ottaviani: None. J. Jackson: None. A.T. Moore: None. K. Ziaka: None. K. Hau: None. A. Lane: None. M.
Michaelides: None. M.E. Cheetham: None. A.J. Hardcastle: None.

\section{C28 Late breaking abstracts}

C28.1

Whole exome sequencing and characterization of coding variation in 49,960 individuals in the UK Biobank

C. Van Hout ${ }^{1}$, I. Tachmazidou ${ }^{2}$, J. Backman', J. Hoffman ${ }^{3}$, B. Ye ${ }^{I}$, A. Pandey ${ }^{3}$ C. Gonzaga-Jauregui, ${ }^{1}$, S. Khalid' ${ }^{1}$ D. Liu ${ }^{1}$, N. Banerjee ${ }^{1}, A . L^{1}$, C. O'Dushlaine', A. Marcketta ${ }^{1}$, J. Staples ${ }^{I}$, C. Schurmann ${ }^{1}$, A. Hawes ${ }^{I}$, E. Maxwell, L. Barnard ${ }^{1}$, A. Lopez ${ }^{I}, J$. Penn $^{I}$, L. Habegger ${ }^{1}$, A. Blumenfeld ${ }^{I}$, A. Yadav ${ }^{1}$, K. Praveen ${ }^{I}$, M. Jones ${ }^{I}$, W. Salerno ${ }^{1}$, W. K. Chung ${ }^{4}$, I. Surakka ${ }^{5}$, C. Willer ${ }^{5}$, K. Hveem $^{6}$, J. Leader ${ }^{7}$, D. Carey ${ }^{7}$, D. Ledbetter ${ }^{7}$, L. Cardon ${ }^{3}$, G. Yancopoulos ${ }^{8}$, A. Economides ${ }^{8}$, G. Coppola ${ }^{1}$, A. Shuldiner ${ }^{1}$, S. Balasubramanian ${ }^{1}$, M. Cantor ${ }^{1}$, M. Nelson, J. Whittaker ${ }^{2}$, J. Reid ${ }^{1}$, J. Marchini ${ }^{1}$, J. Overton ${ }^{1}$, R. Scott ${ }^{2}$, G. Abecasis ${ }^{1}$, L. Yerges-Armstrong ${ }^{3}$, A. Baras ${ }^{1}$

${ }^{1}$ Regeneron Genetics Center, Tarrytown, NY, United States, ${ }^{2}$ GlaxoSmithKline, Brentford, United Kingdom, ${ }^{3}$ GlaxoSmithKline, Philadelphia, PA, United States, ${ }^{4}$ Columbia University Irving Medical Center, New York, NY, United States, ${ }^{5}$ University of Michigan, Ann Arbor, MI, United States, ${ }^{6}$ Norwegian University of Science and Technology, Levanger, Norway, ${ }^{7}$ Geisinger, Danville, PA, United States, ${ }^{8}$ Regeneron Pharmaceuticals, Tarrytown, $N Y$, United States

The UK Biobank is a prospective study of 502,543 individuals, combining extensive phenotypic and genotypic data with streamlined access for researchers around the world. Here we describe the first tranche of large-scale exome sequence data for 49,960 study participants, revealing approximately 4 million coding variants (of which $\sim 98.4 \%$ have frequency $<1 \%$ ). The data includes 231,631 predicted loss of function variants, a $>10$-fold increase compared to imputed sequence for the same participants. Nearly all genes $(>97 \%)$ had $\geq 1$ predicted loss of function carrier, and most genes $(>69 \%)$ had $\geq 10$ loss of function carriers. We illustrate the power of characterizing loss of function variation in this large population through association analyses across 1,741 phenotypes. In addition to replicating a range of established associations, we discover novel loss of function variants with large effects on disease traits, including PIEZOI on varicose veins, COL6Al on corneal resistance, MEPE on bone density, and IQGAP2 and $G M P R$ on blood cell traits. We further demonstrate the value of exome sequencing by surveying the prevalence of 
pathogenic variants of clinical significance in this population, finding that $2 \%$ of the population has a medically actionable variant. Additionally, we leverage the phenotypic data to characterize the relationship between rare BRCAl and $B R C A 2$ pathogenic variants and cancer risk. Exomes from the first 49,960 participants are now made accessible to the scientific community and highlight the promise offered by genomic sequencing in large-scale populationbased studies.

C. Van Hout: A. Employment (full or part-time); Significant; Regeneron Genetics Center. E. Ownership Interest (stock, stock options, patent or other intellectual property); Significant; Regeneron Pharmaceuticals. I. Tachmazidou: A. Employment (full or part-time); Significant; GlaxoSmithKline. J. Backman: A. Employment (full or parttime); Significant; Regeneron Genetics Center. J. Hoffman: A. Employment (full or part-time); Significant; GlaxoSmithKline. B. Ye: A. Employment (full or part-time); Significant; Regeneron Genetics Center. A. Pandey: A. Employment (full or part-time); Significant; GlaxoSmithKline. C. Gonzaga-Jauregui: A. Employment (full or part-time); Significant; Regeneron Genetics Center. S. Khalid: A. Employment (full or part-time); Significant; Regeneron Genetics Center. D. Liu: A. Employment (full or part-time); Significant; Regeneron Genetics Center. N. Banerjee: A. Employment (full or part-time); Significant; Regeneron Genetics Center. A. Li: A. Employment (full or part-time); Significant; Regeneron Genetics Center. C. O'Dushlaine: A. Employment (full or part-time); Significant; Regeneron Genetics Center. A. Marcketta: A. Employment (full or part-time); Significant; Regeneron Genetics Center. J. Staples: A. Employment (full or parttime); Significant; Regeneron Genetics Center. C. Schurmann: A. Employment (full or part-time); Significant; Regeneron Genetics Center. A. Hawes: A. Employment (full or part-time); Significant; Regeneron Genetics Center. E. Maxwell: A. Employment (full or part-time); Significant; Regeneron Genetics Center. L. Barnard: A. Employment (full or part-time); Significant; Regeneron Genetics Center. A. Lopez: A. Employment (full or parttime); Significant; Regeneron Genetics Center. J. Penn: A. Employment (full or part-time); Significant; Regeneron Genetics Center. L. Habegger: A. Employment (full or part-time); Significant; Regeneron Genetics Center. A. Blumenfeld: A. Employment (full or part-time); Significant; Regeneron Genetics Center. A. Yadav: A. Employment (full or part-time); Significant; Regeneron Genetics Center. K. Praveen: A. Employment (full or parttime); Significant; Regeneron Genetics Center. M. Jones: A. Employment (full or part-time); Significant; Regeneron Genetics Center. W. Salerno: A. Employment (full or parttime); Significant; Regeneron Genetics Center. W.K. Chung: F. Consultant/Advisory Board; Significant;
Regeneron Genetics Center. I. Surakka: None. C. Willer: None. K. Hveem: None. J. Leader: None. D. Carey: None. D. Ledbetter: None. L. Cardon: A. Employment (full or part-time); Significant; BioMarin. G. Yancopoulos: A. Employment (full or part-time); Significant; Regeneron Pharmaceuticals. A. Economides: A. Employment (full or part-time); Significant; Regeneron Pharmaceuticals. G. Coppola: A. Employment (full or part-time); Significant; Regeneron Genetics Center. A. Shuldiner: A. Employment (full or part-time); Significant; Regeneron Genetics Center. S. Balasubramanian: A. Employment (full or part-time); Significant; Regeneron Genetics Center. M. Cantor: A. Employment (full or part-time); Significant; Regeneron Genetics Center. M. Nelson: A. Employment (full or parttime); Significant; GlaxoSmithKline. J. Whittaker: A. Employment (full or part-time); Significant; GlaxoSmithKline. J. Reid: A. Employment (full or part-time); Significant; Regeneron Genetics Center. J. Marchini: A. Employment (full or part-time); Significant; Regeneron Genetics Center. J. Overton: A. Employment (full or parttime); Significant; Regeneron Genetics Center. R. Scott: A. Employment (full or part-time); Significant; GlaxoSmithKline. G. Abecasis: A. Employment (full or parttime); Significant; Regeneron Genetics Center. L. YergesArmstrong: A. Employment (full or part-time); Significant; GlaxoSmithKline. A. Baras: A. Employment (full or parttime); Significant; Regeneron Genetics Center.

\section{C28.2}

A novel ciliary Joubert Syndrome-associated protein module regulates axonemal post translational modifications and cilium stability

\section{B. Latour ${ }^{1}$, T. Rusterholz ${ }^{2}$, J. Van de Weghe ${ }^{3}$, M. Gesemann ${ }^{4}$, S. C. F. Neuhauss ${ }^{4}$, D. Doherty, R. Roepman ${ }^{1}$, R. Bachmann-Gagescu ${ }^{2}$}

${ }^{1}$ Radboud University Medical Center, Nijmegen, Netherlands, ${ }^{2}$ University of Zurich- Medical Genetics, Zürich, Switzerland, ${ }^{3}$ University of Washington, Seattle, WA, United States, ${ }^{4}$ University of Zurich- Molecular Life Sciences, Zürich, Switzerland, ${ }^{5}$ University of Washington, Seattle, WA, United States

Joubert Syndrome (JBTS) is a recessive neurodevelopmental ciliopathy, characterized by a pathognomonic hindbrain malformation with variable presence of additional ciliopathy phenotypes affecting kidneys, retina or skeleton. To date, $>35$ genes have been linked to JBTS, but for many families the genetic cause remains unknown. All JBTSgenes encode proteins involved in the structure or function of the primary cilium, an antenna-like organelle essential for signal transduction. A majority of JBTS-proteins form 
complexes at the ciliary transition zone, which acts as a gatekeeper controlling ciliary protein content. Recently, we identified $A R M C 9$, encoding a non-transition zone protein, as a new JBTS gene. In this work, we utilize tandem-affinity purification and yeast two-hybrid screens to find protein interactors of ARMC9. We identify a novel ciliary module composed of two known JBTS-proteins (ARMC9, CEP104) and two proteins not currently associated with human disorders (TOGARAM1, CCDC66). We find variants in TOGARAM1 in individuals with JBTS, and show that variants located in the TOG2 domain disrupt the interaction with ARMC9. We generate CRISPR-edited armc 9 and togaraml zebrafish mutants which display reduced numbers of shortened cilia and typical ciliopathy phenotypes including curved bodies and pronephric cysts. Patient fibroblasts with ARMC9 or TOGARAM1 mutations display ciliary abnormalities with axonemal shortening and reduced levels of post-translational modifications (acetylation and polyglutamylation), which are also found in our zebrafish models. Moreover, ciliary resorption induced by cold or by cell cycle reentry is affected in ARMC9 and TOGARAM1 mutant cell lines, suggesting a role for this new JBTSassociated complex in axonemal microtubule stability.

B. Latour: None. T. Rusterholz: None. J. Van de Weghe: None. M. Gesemann: None. S.C.F. Neuhauss: None. D. Doherty: None. R. Roepman: None. R. Bachmann-Gagescu: None.

\section{C28.3}

Index and vocabulary of accessible DNA elements in the human genome

\section{W. Meuleman, S. Muratov, E. Rynes, J. Stamatoyannopoulos}

Altius Institute for Biomedical Sciences, Seattle, WA, United States

Regulatory information encoded in the human genome is activated by sequence-specific DNA binding factors, creating focal alterations in chromatin structure that are hypersensitive to DNase I. We created deep reference maps of DNase I hypersensitive sites (DHSs) from 733 human biosamples encompassing 439 cell and tissue types and states, and integrated these to precisely delineate and numerically index $\sim 3.6$ million DHSs encoded within the human genome, providing a common coordinate system for regulatory DNA. Here we show that the expansive scale of cell and tissue states sampled exposes an unprecedented degree of stereotyped actuation of large sets of elements, signaling the operation of distinct genome-scale regulatory programs. We show further that the complex actuation patterns of individual elements can be captured comprehensively by a simple regulatory vocabulary reflecting their dominant program. This vocabulary, in turn, enables comprehensive and quantitative regulatory annotation of both protein-coding genes and the vast array of welldefined but poorly-characterized non-coding RNA genes. Finally, we show that regulatory vocabularies open new avenues for systematically interpreting non-coding genetic variation, and substantially empower the connection of disease-associated variation with specific cell and tissue states. Taken together, our results provide a common and extensible coordinate system and vocabulary for human regulatory DNA, and open a new global perspective on the architecture of human gene regulation.

W. Meuleman: None. S. Muratov: None. E. Rynes: None. J. Stamatoyannopoulos: None.

\section{C28.4}

Modeling the pathological long-range regulatory effects of human structural variation with patient-specific hiPSCs

M. Laugsch ${ }^{1,2,3}$, M. Bartsuel ${ }^{1}$, R. Rehimi ${ }^{1}$, H. Alirzayeva ${ }^{1}$, A. Karaolidou ${ }^{1}$, G. Crispatzu ${ }^{1}$, P. Zentis ${ }^{4}$, M. Nikolic ${ }^{1}$, T. Bleckwehl, P. Kolovos ${ }^{5}$, W. F. J. van Ijcken ${ }^{6}$, T. Saric ${ }^{7}$, K. Koehler ${ }^{8}$, P. Frommolt ${ }^{4}$ C. Schaaf ${ }^{2,3}$, K. Lachlan ${ }^{9,10,}$ J. Baptista ${ }^{11,12}$, A. Rada-Iglesias $1,4,13$

${ }^{I}$ Centre for Molecular Medicine Cologne (CMMC), University of Cologne, Cologne, Germany, ${ }^{2}$ Institute of Human Genetics, University Hospital, Cologne, Germany, ${ }^{3}$ Institute of Human Genetics, University Hospital Heidelberg, Heidelberg, Germany, ${ }^{4}$ Cluster of Excellence Cellular Stress Responses in Aging-Associated Diseases (CECAD), University of Cologne, Cologne, Germany, ${ }^{5}$ Biotech Research and Innovation Centre (BRIC), University of Copenhagen, Copenhagen, Denmark, ${ }^{6}$ Erasmus Medical Centre, University of Rotterdam, Rotterdam, Netherlands, ${ }^{7}$ Center for Physiology and Pathophysiology, Institute for Neurophysiology, University of Cologne, Cologne, Germany, ${ }^{8}$ Department of Pediatrics, University Clinic Carl Gustav Carus, Dresden, Germany, ${ }^{9}$ Human Genetics and Genomic Medicine, Southampton General Hospital, University of Southampton, Southampton, United Kingdom, ${ }^{10}$ Clinical Genetics Service, University Hospital Southampton NHS Foundation Trust, Princess Anne Hospital, Southampton, United Kingdom, ${ }^{11}$ Molecular Genetics Department, Royal Devon and Exeter NHS Foundation Trust, Exeter, United Kingdom, ${ }^{12}$ Institute of Biomedical and Clinical Science, University of Exeter, Medical School, Exeter, United Kingdom, ${ }^{13}$ Institute of Biomedicine and Biotechnology of Cantabria (IBBTEC), University of Cantabria, Cantabria, Spain 
Elucidating the pathological consequences of disrupting 3D genome organization is often difficult to achieve in vivo due to differences in gene dosage sensitivity between mice and humans. This is well illustrated by Branchiooculofacial Syndrome (BOFS), a rare congenital disorder mainly characterized by craniofacial malformations and orofacial cleft (OC). BOFS is caused by heterozygous mutations within TFAP2A, a master regulator of neural crest cells (NCC) and craniofacial development for which humans, but not mice, are haploinsufficient. Here we present a BOFS patient carrying a heterozygous inversion with one breakpoint located downstream of TFAP2A. As several heterozygous translocations breakpoints and risk haplotypes located downstream of TFAP2A were previously also associated with $O C$ we asked if this region is important for TFAP2A expression in NCC derived from hiPSCs.

Using CRISPR/Cas9 technology we showed that TFAP2A is located within a Topologically Associating Domain (TAD) containing enhancers that are essential for TFAP2A expression and NCC development. Moreover, in NCC derived from patient-specific hiPSCs the inversion disconnects one TFAP2A allele from its cognate enhancers, leading to monoallelic and haploinsufficient TFAP $2 A$ expression. Although the inversion shuffles within the same TAD the TFAP2A NCC enhancers with potentially novel target genes, this does not result in enhancer adoption.

Our work illustrates the power of combining patientspecific hiPSCs differentiation with different genomic and genetic engineering tools to overcome current limitations in unveiling the long-range pathological consequences of human structural variation.

This work was primarily supported by CMMC intramural funding, Fritz Thyssen Stiftung, Else Kröner Fresenius Stiftung and DFG.

M. Laugsch: None. M. Bartsuel: None. R. Rehimi: None. H. Alirzayeva: None. A. Karaolidou: None. G. Crispatzu: None. P. Zentis: None. M. Nikolic: None. T. Bleckwehl: None. P. Kolovos: None. W.F.J. van Ijcken: None. T. Saric: None. K. Koehler: None. P. Frommolt: None. C. Schaaf: None. K. Lachlan: None. J. Baptista: None. A. Rada-Iglesias: None.

\section{C28.5}

Cerebral organoids provide insights into brain neurodevelopment disrupted by the 16p11.2 copy number variants in autism

L. Iakoucheva ${ }^{1}$, J. Urresti ${ }^{1}$, P. Zhang ${ }^{1}$, P. Moran Losada ${ }^{1}$,

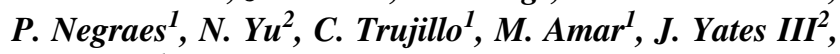
A. Muotri ${ }^{1}$
${ }^{1}$ University of California San Diego, La Jolla, CA, United States, ${ }^{2}$ Scripps Research Institute, La Jolla, CA, United States

Introduction: The $16 \mathrm{p} 11.2$ is the most common among all rare copy number variants (CNVs) associated with Autism Spectrum Disorder (ASD). We investigated neurodevelopmental processes impacted by the dosage changes of 16p11.2 CNV using cerebral organoid models.

Materials and Methods: We generated 3D cultures (i.e. human cerebral organoids) that model human fetal brain development using iPSCs derived from skin fibroblasts of $16 \mathrm{p} 11.2$ deletion (DEL) and duplication (DUP) carriers. Transcriptome sequencing, proteomic profiling and immunofluorescence were carried out to investigate molecular and cellular impact of the $16 \mathrm{p} 11.2 \mathrm{CNV}$. Cell migration, neuronal morphology and synaptogenesis were also investigated.

Results: Cerebral organoid recapitulated macrocephaly and microcephaly phenotypes observed in 16p11.2 DEL and DUP carriers. Increased proliferation of neuroprogenitor cells was observed in DELs. Gene co-expression network analyses identified co-expression modules that were strongly associated with $16 \mathrm{p} 11.2$ copy number status. A module enriched in genes with cell migration and locomotion functions was downregulated in DELs. A module enriched in genes with synaptic functions, was upregulated in DELs. Severe migration defects in DEL organoids were confirmed by Boyden chamber assay. Neurons derived from DEL organoids had longer neurites and increased soma size. The number of functional synapses was also higher in DEL organoids. Treatment with the RhoA inhibitor rhosin rescued cell migration defects.

Conclusion: Cerebral organoids recapitulate patients head size phenotypes and gene expression profiles of fetal human brain. Genes associated with cell migration and synaptic functions were dysregulated by the $16 \mathrm{p} 11.2 \mathrm{CNV}$. These molecular findings were validated experimentally and rescued by a small molecule inhibitor.

L. Iakoucheva: None. J. Urresti: None. P. Zhang: None. P. Moran Losada: None. P. Negraes: None. N. Yu: None. C. Trujillo: None. M. Amar: None. J. Yates III: None. A. Muotri: None.

\section{C28.6}

Preclinical validation of allele-specific silencing in patient-derived directly reprogrammed neuronal cell lines as effective treatment for Autosomal Dominant LeukoDystrophy

E. Giorgio ${ }^{\text {, M. Morenzati }}{ }^{2}$, P. Rivetti di Val Cervo ${ }^{3}$, A. Brussino ${ }^{1}$, M. Cernigoj ${ }^{3}$, E. Della Sala ${ }^{1}$, A. Bartoletti

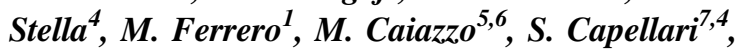


P. Cortelli, ${ }^{4,8}$, L. Conti ${ }^{9}$ E. Cattaneo ${ }^{3,10}$, A. Buffo $^{2}$, A. Brusco ${ }^{1,11}$

${ }^{1}$ University of Torino-Dep Medical Sciences, Torino, Italy, ${ }^{2}$ University of Torino-Department of Neuroscience Rita Levi Montalcini and Neuroscience Institute Cavalieri Ottolenghi (NICO), Torino, Italy, ${ }^{3}$ University of MilanoDepartment of Biosciences, Laboratory of Stem Cell Biology and Pharmacology of Neurodegenerative Diseases, Milano, Italy, ${ }^{4}$ IRCCS Istituto delle Scienze Neurologiche di Bologna, Bellaria Hospital, Bologna, Italy, ${ }^{5}$ Department of Pharmaceutics, Utrecht Institute for Pharmaceutical Sciences (UIPS), Utrecht, Netherlands, ${ }^{6}$ Department of Molecular Medicine and Medical Biotechnology, University of Naples "Federico, Napoli, Italy, ${ }^{7}$ University of Bologna, Department of Biomedical and Neuromotor Sciences, Torino, Italy, ${ }^{8}$ University of Bologna, Department of Biomedical and Neuromotor Sciences, Bologna, Italy, ${ }^{9}$ University of Trento, Centre for Integrative Biology (CIBIO), Laboratory of Computational Oncology, Trento, Italy, ${ }^{10}$ National Institute of Molecular Genetics (INGM) Romeo and Enrica Invernizzi, Milano, Italy, ${ }^{11}$ Città della Salute e della Scienza University Hospital, Medical Genetics Unit, Torino, Italy

Allele-SPecific silencing by RNA Interference (ASP-RNAi) holds promise as a therapeutic strategy for gain of function and repeat-expansion diseases. Here, we propose ASPRNAi as effective therapeutic option also for genetic disorders associated with gene/s duplication. As a proofof-concept, we studied Autosomal Dominant adult-onset demyelinating LeukoDystrophy (ADLD), a hereditary, progressive and fatal disorder caused by Lamin B1 (LMNB1) duplication. We have identified three ASPsiRNAs able to specifically and effectively silence the non-duplicated allele of $L M N B 1$, maintaining transcriptionally active only two alleles as in healthy subjects, restoring its physiological mRNA/protein levels and ameliorating disease-specific cellular phenotypes in ADLD fibroblasts. To determine ASP-silencing efficacy in cell types more relevant to the disease, we generated neurons directly reprogrammed from human fibroblasts, and used rat oligodendrocyte precursor cells (OPCs) overexpressing the human $L M N B 1$. We initially proved that these cellular models presented ADLD-specific cellular phenotypes, supporting their relevance as in vitro preclinical tools. Then, we treated both cellular models with the selected most efficient ASP-siRNA at two different time-points. The treatment reduced LMNB1 protein level and ameliorated ADLD-specific cellular abnormalities (e.g., nuclear anomalies and neurite growth), further validating the therapeutic potential of our RNA molecule and its allele-specificity. Overall, we demonstrated that ASP-RNAi is a suitable and promising therapeutic option for ADLD, prompting further in vivo studies to validate our results before a clinical trial. Moreover, our results have a broad translational value since our strategy can be extended to all pathological conditions associated with gene copy number gain.

E. Giorgio: None. M. Lorenzati: None. P. Rivetti di Val Cervo: None. A. Brussino: None. M. Cernigoj: None. E. Della Sala: None. A. Bartoletti Stella: None. M. Ferrero: None. M. Caiazzo: None. S. Capellari: None. P. Cortelli: None. L. Conti: None. E. Cattaneo: None. A. Buffo: None. A. Brusco: None.

\section{C29 Stakeholder perspectives in cancer genetics}

\section{C29.1}

The public favours healthcare-mediated disclosure of hereditary cancer risk to at-risk relatives: a populationbased survey in Sweden

\section{A. Andersson ${ }^{1}$, C. Hawranek ${ }^{1}$, A. Öfverholm ${ }^{2}$, H. Ehrencrona ${ }^{3}$, K. Grill ${ }^{4}$, S. Hajdarevic ${ }^{5}$, B. Melin ${ }^{1}$, E. Tham ${ }^{6}$, B. Numan Hellquist ${ }^{1}$, A. Rosén ${ }^{1}$}

${ }^{1}$ Department of Radiation sciences, Oncology, Umeå university, Umeå, Sweden, ${ }^{2}$ Department of Clinical sciences, Oncology, University of Gothenburg, Gothenburg, Sweden, ${ }^{3}$ Department of Clinical Genetics, Lund University, Lund, Sweden, ${ }^{4}$ Department of historical, philosophical and religious studies, Philosophy, Umeå university, Umeå, Sweden, ${ }^{5}$ Department of Nursing, Umeå University, Umeå, Sweden, ${ }^{6}$ Department of Molecular Medicine and Surgery, Karolinska Institutet, Stockholm, Sweden

Introduction: Family-mediated information to relatives at risk is the current Swedish clinical practice in cancer genetics. We addressed individuals from the general population to explore interest in being informed if a cancer genetic investigation, revealing an increased cancer risk, had been conducted in their family. We also investigated preferences on source and mode of information.

Materials and Methods: A random sample of the general public was approached through a Swedish citizen webpanel during October 2018. Respondents were given the scenarios of being member of a family with an increased risk of colorectal cancer (CRC), 10 and 70\% lifetime risk, and being a potential at-risk relative. Regular colonoscopy was presented as a preventive measure to individuals at risk.

Results: Out of 1900 invited, 977 responded $(51 \%)$. For the scenario of 10 and $70 \%$ CRC-risk, 89.2 and $90.6 \%$ respectively, would like to receive information about the investigation. The proportion preferring to be informed was higher among women $(91.5 \%, 93.3 \%)$ than men $(85.7 \%$, 
$88.2 \%)$, $($ Chi2, $\mathrm{p}=0.044, \mathrm{p}=0.047)$. There was no significant difference by age, educational level, place of residence or parity.

Regarding source of the information, 79.7 and $74.9 \%$ respectively would prefer to receive the information from health care and 16.9 and $19.1 \%$ from a family member.

Conclusion: In this study a majority wanted to be informed about a completed cancer genetic investigation and preferred health care professionals to mediate this information. The presented lifetime risk did not affect the respondents' interest in being informed.

Grants: Cancer research foundation in Norrland and Forte.

A. Andersson: None. C. Hawranek: None. A. Öfverholm: None. H. Ehrencrona: None. K. Grill: None. S. Hajdarevic: None. B. Melin: None. E. Tham: None. B. Numan Hellquist: None. A. Rosén: None.

\section{C29.2}

Communication across generations: disclosure of BRCA cancer risk with young adults

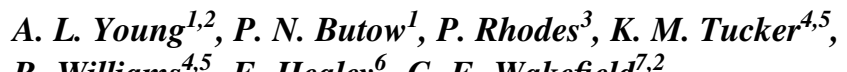
R. Williams $^{4,5}$, E. Healey $^{6}$, C. E. Wakefield ${ }^{7,2}$

${ }^{1}$ Centre for Medical Psychology \& Evidence-based Decision-making (CeMPED), School of Psychology, The University of Sydney, Sydney, Australia, ${ }^{2}$ Behavioral Sciences Unit Proudly Supported by the Kids with Cancer Foundation, Kids Cancer Centre, Sydney Children's Hospital, Sydney, Australia, ${ }^{3}$ School of Psychology, The University of Sydney, Sydney, Australia, ${ }^{4}$ Hereditary Cancer Clinic, Department of Medical Oncology, Prince of Wales Hospital, Sydney, Australia, ${ }^{5}$ Prince of Wales Clinical School, University of New South Wales, Sydney, Australia, ${ }^{6}$ Illawarra Shoalhaven Cancer \& Haematology Network, Wollongong Hospital, Sydney, Australia, ${ }^{7}$ School of Women's and Children's Health, University of New South Wales, Sydney, Australia

Background: Family communication is vital to ensure dissemination of genetic information to at-risk relatives and to facilitate supportive family interactions around genetic testing and risk management decisions. Yet, little is known about how young adults and their families perceive and manage these interactions.

Materials and Methods: Analysis was based on family systems theory using a mixed-method approach: family interviews ( $\mathrm{N}=68$ individuals; 21 families), young adults interviews $(\mathrm{N}=33)$, pedigree data and standardized measures.

Results: Six key themes were identified and explored: 1) Responsibility to protect, 2) "It's a woman's problem", 3)
Family culture influences communication, 4) Adversarial growth and connection, 5) Key events can be relational turning points, and 6) Health professionals can help. Family identities were solidified through the incorporation of a pathogenic variant in family scripts, while members of the family who held differing views to their families expressed less agreeableness and openness to disseminate information. The collective family's experience and perspective towards a pathogenic variant can influence a young adult's decisionmaking about genetic testing, risk-management and family planning. The utilization of family therapy skills in routine practice would be helpful in facilitating communication and the inclusion of standardized measures is beneficial to identify individuals needing ongoing psychological support.

Conclusion: Understanding relationship difficulties that arise from family members holding family-divergent views offers insight for future research inquiry and areas of further training and clinical support.

A.L. Young: None. P.N. Butow: None. P. Rhodes: None. K.M. Tucker: None. R. Williams: None. E. Healey: None. C.E. Wakefield: None.

C29.3

High-Risk Women's Responses and Understanding of Polygenic Breast Cancer Risk Information

T. Yanes ${ }^{1}$, R. Kaur ${ }^{\text {I, B. Meiser }}{ }^{\text {I, M. Scheepers-Joynt }}{ }^{2}$, S. McInerny ${ }^{2}$, K. Barlow-Stewart ${ }^{3}$, Y. Antill', L. Salmon ${ }^{5}$,

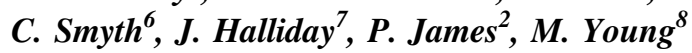

${ }^{1}$ University of New South Wales, Sydney, Australia, ${ }^{2}$ Parkville Familial Cancer Centre, Melbourne, Australia, ${ }^{3}$ University of Sydney, Sydney, Australia, ${ }^{4}$ Cabrini Health, Melbourne, Australia, ${ }^{5}$ Austin Hospital, Melbourne, Australia, ${ }^{6}$ Monash Medical Centre, Melbourne, Australia, ${ }^{7}$ Murdoch Children's Research Institute, Melbourne, Australia, ${ }^{8}$ Kinghorn Centre for Clinical Genomics, Sydney, Australia

Background: It is estimated that polygenic risk scores (PRS) can explain up to $18 \%$ of familial breast cancer. Implementation of polygenic testing has begun, with several commercial laboratories now offering the test. Despite implementation, there is little research investigating how women respond and understand PRS. This study aimed to explore women's experience receiving their personalized breast cancer PRS and compare responses of women at different levels of polygenic risk.

Methods: Eligible participants were affected and unaffected women from families clinically assessed to be at high-risk for breast cancer who had received their personalized PRS as part of the Variants in Practice Psychosocial Study (ViPPs). In-depth semi-structured interviews were 
conducted up to four weeks after receiving PRS. Interviews were transcribed verbatim and analysed using thematic analysis.

Results: Twenty-one women participated in this qualitative study (mean age 53.4 years). Eleven women received a PRS that was in the top quartile of PRS distribution and 10 in the lowest quartile. Women's lived experience with breast cancer informed how they responded to their PRS, constructed and made sense of breast cancer risk following receipt of their PRS, and how they integrated this new information into their breast cancer risk management. Regardless of risk level, all participants demonstrated broad knowledge of concepts related to polygenic information and were able to accurately describe the implications of their PRS. Receiving PRS did not appear to negatively impact women's distress levels.

Conclusion: Polygenic breast cancer information is well received and understood by women at high-risk for breast cancer.

T. Yanes: None. R. Kaur: None. B. Meiser: B. Research Grant (principal investigator, collaborator or consultant and pending grants as well as grants already received); Modest; Astrazeneca. B. Research Grant (principal investigator, collaborator or consultant and pending grants as well as grants already received); Significant; Has received funding from Astrazeneca for a study unrelated to this project. M. Scheepers-Joynt: None. S. McInerny: None. K. BarlowStewart: None. Y. Antill: None. L. Salmon: None. C. Smyth: None. J. Halliday: None. P. James: None. M. Young: None.

\section{C29.4}

Families' and healthcare professionals' uncertainties in the era of cancer precision medicine: results from PRISM

\section{J. Vetsch ${ }^{1,2}$, C. E. Wakefield ${ }^{1,2}$, M. Warby ${ }^{3}$, K. Tucker ${ }^{3,4}$, E. Duve, , G. Marshall ${ }^{2,5,6}$, T. Trahair ${ }^{2,5}$, T. O'Brien ${ }^{2,5}$, L. Lau $^{2,5}$, D. Ziegler ${ }^{2,5}$}

${ }^{1}$ Behavioural Sciences Unit, Kids Cancer Centre, Sydney Children's Hospital, Randwick, Australia, ${ }^{2}$ School of Women's and Children's Health, UNSW Sydney, Kensington, Australia, ${ }^{3}$ Hereditary Cancer Centre, Prince of Wales Hospital, Randwick, Australia, ${ }^{4}$ Prince of Wales Clinical School, UNSW Sydney, Randwick, Australia, ${ }^{5}$ Kids Cancer Centre, Sydney Children's Hospital, Randwick, Australia, ${ }^{6}$ Children's Cancer Institute, Lowy Cancer Centre, UNSW, Kensington, Australia

Background: The PRISM precision medicine trial uses novel technologies to personalize treatment for children with high-risk cancer. Families and healthcare professionals can face uncertainties regarding likely treatment outcomes and implications of genomic findings. 'PRISM-Impact' assesses families' and healthcare professionals' experiences with PRISM.

Methods: Patients aged $<21$ years with a high-risk cancer are eligible for PRISM. Parents, patients ( $>12$ years), and healthcare professionals opt-in to PRISM-Impact, complete surveys at enrolment, after results delivery, and then annually for five years. We interview healthcare professionals annually.

Results: Currently, 95 families have opted-in and 76 parents (child's mean age: 8.68 years [range $=0-17$ ]; $67.1 \%$ mothers) have returned surveys. We have interviewed 54 healthcare professionals (59\% oncologists). Parents' expectations of benefit vary widely, possibly due to feelings of uncertainty, with some reporting that their child is 'extremely likely' to benefit (7.9\%) while others report there is 'no chance at all' of benefit $(6.6 \%)$. This contrasts with healthcare professionals' expectations, with only $18.5 \%$ of professionals ranking 'current patients' as the stakeholder benefitting most from PRISM. Parents' intolerance of uncertainty ranged from 12-51 (out of 60) and parents' anxiety was high, on average $4.9(\mathrm{SD}=2.99$, range $0-10)$. Parents' intolerance of uncertainty and anxiety were highly correlated $(\mathrm{r}=0.555, \mathrm{p}<0.001)$.

Conclusion: Parents with higher intolerance of uncertainty might be at greater risk of distress and may benefit from targeted psychosocial support. It will be important to analyze parents' emotional reactions and coping after PRISM results delivery, and to assess whether their coping is associated with their baseline (in)tolerance of uncertainty.

J. Vetsch: None. C.E. Wakefield: None. M. Warby: None. K. Tucker: None. E. Duve: None. G. Marshall: None. T. Trahair: None. T. O'Brien: None. L. Lau: None. D. Ziegler: None.

\section{C29.5}

Advanced cancer patient perspectives on consenting to molecular tumour profiling

M. Best ${ }^{1}$, P. Butow ${ }^{2}$, N. Bartley ${ }^{2}$, C. Jacobs ${ }^{3}$, I. Juraskova ${ }^{2}$, A. Newson ${ }^{1}$, D. Goldstein ${ }^{4}$, J. Savard ${ }^{5}$, B. Meiser ${ }^{6}$, M. Ballinger ${ }^{7}$, B. Biesecker ${ }^{8}$, D. Thomas $^{7}$

${ }^{1}$ University of Sydney, Sydney, Australia, ${ }^{2}$ School of Psychology, University of Sydney, Australia, ${ }^{3}$ University of Technology, Sydney, Australia, ${ }^{4}$ Prince of Wales Clinical School, Sydney, Australia, ${ }^{5}$ Deakin University, Geelong, Australia, ${ }^{6}$ University of NSW, Sydney, Australia, ${ }^{7}$ Garvan Institute of Medical Research, Sydney, Australia, ${ }^{8}$ RTI International, Washington, DC, United States 
Molecular tumour profiling (MTP), aiming to link molecular targets in tumours to cognate therapies, has entered clinical practice. However, little is yet known about the psychosocial and behavioural implications of MTP. The Molecular Screening and Therapeutics program is recruiting 1,000 patients with an advanced, solid, rare cancer to undertake MTP and, if an actionable variant is found, participate in a suitable therapeutic trial if available. The current mixed methods psychosocial sub-study (PiGeOn) aims to explore participants' understanding, experiences and views regarding MTP at three timepoints. Baseline qualitative results are reported here. PiGeOn participants were purposively sampled to participate in a semi-structured interview. Thematic analysis is employed to determine themes. Data saturation was reached at 20 (aged 41-77 years). Participants' motivations include: fear of death, trust in their oncologist, need for control and a sense of 'nothing lost'. Desperation and fear of dying dominate motivation, but participants also value being able to help others. Participants tolerate uncertainty, hope for reduced prognostic uncertainty, but fear loss of hope if no actionable result is found (the most likely outcome). They often lack understanding, but trust the science of MTP and the research process. This is one of few studies to explore the experiences of people who have undergone MTP. Identifying new treatment options overshadows broader and familial implications of genetic testing in this population. This increases the difficulty of supporting informed consent and shared decision-making in MTP testing. The current data will inform future policy and practice. Funded by NHMRC.

M. Best: None. P. Butow: None. N. Bartley: None. C. Jacobs: None. I. Juraskova: None. A. Newson: None. D. Goldstein: None. J. Savard: None. B. Meiser: None. M. Ballinger: None. B. Biesecker: None. D. Thomas: None.

\section{C29.6}

Dimensions of grief and loss for families living with $\mathrm{Li}$ Fraumeni Syndrome

\author{
A. Werner-Lin ${ }^{1}$, S. L. Merrill', J. L. Young',

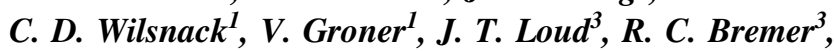 \\ J. A. Peters $^{3}$, M. H. Greene ${ }^{3}$, S. A. Savage ${ }^{3}$, \\ P. P. Khincha ${ }^{3}$
}

${ }^{1}$ School of Social Policy and Practice, Philadelphia, PA, United States, ${ }^{2}$ Stanford University Center for Biomedical Ethics, Stanford, CA, United States, ${ }^{3}$ National Cancer Institute, Bethesda, MD, United States

Li-Fraumeni Syndrome (LFS) is an inherited cancer predisposition syndrome associated with germline mutations in TP53. LFS is characterized by early-onset, multiple primary malignancies with nearly $100 \%$ penetrance by age 70 years, and substantial uncertainty regarding diagnostic timing and prognoses. An interdisciplinary team used Interpretive Description to analyze interviews with family groups enrolled in the National Cancer Institute's LFS study (NCT01443468). Interviews elicited narratives about cancer experiences, evolving family structure and functioning, and coping.

Families reported limited reprieve between cancer diagnoses and deaths, which often occurred simultaneously and across generations. Consequently, participants lived with grief as an integral feature of family life. Extensive diseaserelated uncertainty created ambiguous losses, with little guidance to interpret risk or prepare for the next change. Family groups reported anticipatory losses, shared expectations regarding predicted functional and emotional change resulting from a new cancer or death. Individuals reported embodied loss, impairment to physical or aesthetic functioning due to cancer treatment or prevention. These types of grief occurred simultaneously, often went unacknowledged, and were often neither recognized nor discussed with social or provider networks. Unacknowledged losses included survivor guilt, identity, hoped-for life experiences, and altered reproductive plans.

Family experiences with loss may warrant clinical attention over time, as opposed to solely at the time of diagnosis. Traditional medical teams may lack capacity to address the complex psychosocial needs of families grieving LFSrelated losses. Long-term engagement of mental health providers with bereavement training may provide invaluable support to families with LFS and facilitate engagement with providers.

A. Werner-Lin: None. S.L. Merrill: None. J.L. Young: None. C.D. Wilsnack: None. V. Groner: None. J.T. Loud: None. R.C. Bremer: None. J.A. Peters: None. M.H. Greene: None. S.A. Savage: None. P.P. Khincha: None. 